\title{
LA NÉCROPOLE DU NÉOLITHIQUE MOYEN DE SUR LES PÂTUREAUX À CHICHERY (YONNE)
}

\author{
Philippe CHAmbon ${ }^{1}$, Jean-Paul Delor ${ }^{2}$, Anne AUGEREAU ${ }^{3}$, \\ Juan Francisco GibajA BAO ${ }^{4}$, Katia MEUniER ${ }^{3}$, Aline THOMAS $^{5}$ et Pascal MuraIL ${ }^{5}$ \\ avec la collaboration de Danièle MOLEZ ${ }^{1}$
}

À Jean-Paul Delor

\begin{abstract}
Mots-clés. Pratiques funéraires, Néolithique moyen, Cerny, sépultures type Balloy.
Résumé. La nécropole de Sur les Pâtureaux à Chichery a été explorée entre 1981 et 1984 par J.-L. Tainturier puis par J.-P. Delor. Treize sépultures ont été mises au jour sur $600 \mathrm{~m}^{2}$, les limites du gisement n'étant vraisemblablement pas atteintes. Ces sépultures se rattachent essentiellement au type Balloy, reconnu dans une dizaine de gisements du Bassin parisien. Elles associent une architecture à même la fosse et un défunt inséré dans un contenant mobile, et sont attribuées à l'horizon Cerny.

Les tombes sont installées sur deux à trois files dans un axe nord-ouest/sud-est, sans qu'on puisse supposer qu'elles étaient incluses dans un ou plusieurs monuments allongés, comme c'est souvent le cas pour ce type de sépultures.

La population inhumée présente une composition compatible avec une population naturelle. Toutefois la concentration des plus jeunes sujets sur une petite surface peut aussi indiquer une sectorisation de la nécropole. Le mobilier associé aux sépultures est peu abondant et comprend uniquement de l'industrie lithique en silex, parmi laquelle dominent les armatures de flèche et les pics. Aucune trace macroscopique d'usage n'est visible sur les pièces.

Une telle industrie ne dépare pas dans le contexte des sépultures de type Balloy, mais l'absence de tout autre mobilier est inédite. Si le Cerny est inévitablement la première référence pour une telle nécropole, quelques caractères de Sur les Pâtureaux incitent à la prudence. Les pics sont fréquents dans les habitats de cette culture, en revanche certaines armatures renvoient davantage vers le Rössen et Chassey. En outre, l'orientation des sépultures est celle qui prévaut dans le Mittelneolithikum rhénan.

Les sept datations obtenues placent les tombes avant le milieu du Ve millénaire, soit dans la première partie de l'horizon Cerny. Elles assurent que les tombes de type Balloy existent en Bassin parisien dès le début de cet horizon, sans rapport avec l'un ou l'autre des faciès céramiques du Cerny.
\end{abstract}

1. CNRS, UMR 7041: «Archéologies et sciences de l'Antiquité (ArScAn) », équipe Ethnologie préhistorique, Maison René-Ginouvès, 21 allée de l’Université, F-92023, Nanterre Cedex. Courriels: philippe.chambon@mae.u-paris10.fr; daniele.molez@mae.u-paris10.fr

2. UMR 5594: «Archéologie, terre, histoire, sociétés (Artehis)», équipe Anthropisation de l'environnement, Université de Bourgogne, 6 boulevard Gabriel, F-21000 Dijon. Notre collègue, Jean-Paul Delor, n'aura pas vu ces pages publiées. Ni cet article, ni le programme qui le soustend n'auraient vu le jour sans son implication constante dans la recherche archéologique, et sans sa générosité.

3. Inrap, UMR 7041: «Archéologies et sciences de l’Antiquité (ArScAn) », équipe Protohistoire européenne, Maison René-Ginouvès, 21 allée de l’Université, F-92023, Nanterre Cedex. Courriels: anne.augereau@inrap.fr; katia.meunier@mae.u-paris10.fr

4. Universidade do Algarve, Faculdade de Ciências Humanas e Sociais, Departamento de História, Arqueologia e Património, Campus de Gambelas, P-8005-139 Faro. Courriel: jfgibaja@gmail.com

5. Université Bordeaux-I, UMR 5199 - De la Préhistoire à l'Actuel: Culture, Environnement et Anthropologie (PACEA), Université Bordeaux-I, Bâtiment B8, avenue des Facultés, F-33405 Talence Cedex. Courriels: a.thomas@anthropologie.u-bordeaux1.fr; p.murail@ anthropologie.u-bordeaux1.fr 
Key-words. Burial practices, Middle Neolithic, Cerny, "Balloy type" graves.

Abstract. The necropolis of Sur les Pâtureaux in Chichery (Yonne, France) was explored from 1981 to 1984 by J.-L. Tainturier, then by J.-P. Delor. Thirteen graves were uncovered over a surface of $600 \mathrm{~m}^{2}$ while the limits of the site have probably not been attained. Most of these graves are of the "Balloy type", which is identified in around a dozen sites in the Paris Basin. They associate a "vault" installed within the grave and a defunct placed in a mobile container. They are attributed to the Cerny horizon.

The tombs are installed in two to three rows following a north-west/south-east axis, though we cannot assume they were included in one or several elongated monuments, as is often the case for this type of burial.

The population includes a significant number of very young individuals, indicating either that the selection of the subjects was not based on age, or that the necropolis was divided into sectors based on this same criteria. The objects associated with the graves are few and consist only of worked flint objects, mainly arrowheads and picks. No macroscopic traces of use are visible on them.

Such a lithic industry is not incongruous in the context of Balloy-type burials, but the absence of any other objects is elsewhere unknown. While Cerny is undeniably the primary reference for such a necropolis, some features of Sur les Pâtureaux voice caution. Though picks are frequent in the habitats of this culture, some of the weapon armatures are more closely associated with the Rössen and Chassey cultures. Moreover, the orientation of the graves is the one most frequently given in the Mittelneolithikum of Rhineland.

The seven dates obtained situate the tombs before the middle of the $5^{\text {th }}$ millennium, or in the first part of the Cerny horizon. They confirm that Balloy-type tombs exist in the Paris Basin from the very beginning of this horizon, with no relation to the pottery facies of the Cerny culture.

Translation: Magen O'FARRELL

\section{LA FOUILLE DE LA NÉCROPOLE DANS SON CONTEXTE LOCAL ET RÉGIONAL}

Publier une fouille vingt ans après sa réalisation pourrait sembler trop tardif et inutile. À l'époque, la recherche privilégiait l'étude des sites d'habitat et les anthropologues étaient trop peu nombreux pour gérer les nécropoles appréhendées. Il revenait à chaque responsable d'opération de faire la preuve de ses compétences avec des fortunes diverses. Fouiller est une chose, mais interpréter les données issues de la fouille en est une autre. Concernant Sur les Pâtureaux, les données issues de la fouille étaient suffisamment novatrices et sans références directes avec d'autres sites du Bassin parisien pour nécessiter qu'elles soient réservées en attendant «des études complémentaires». À l'évidence, des rapports pouvaient être établis avec le site de Passy sans pour autant en retrouver toutes les caractéristiques.

Les années ont passé et de nouvelles fouilles menées dans l'Auxerrois, sous la direction de véritables spécialistes du funéraire, nous permettent maintenant de reconsidérer ces interventions déjà anciennes avec un autre regard et suivant une autre approche ${ }^{6}$.

6. L'étude et la publication de ce gisement s'insère dans les travaux du Programme collectif de recherche «Évolution, coexistence, confrontation de pratiques funéraires entre 4500 et 4000 av. J.-C. sur un microterritoire: la vallée de l'Yonne, entre le Serein et le Baulche». Le matériel et les archives du gisement sont désormais rassemblés

\section{LOCALISATION GÉOGRAPHIQUE ET CONTEXTE GÉOLOGIQUE}

À une douzaine de kilomètres au nord d'Auxerre, la commune de Chichery est située dans la vallée de l'Yonne à environ $800 \mathrm{~m}$ au sud de sa confluence avec le Serein, puis à presque $4 \mathrm{~km}$ de sa confluence avec l'Armançon (fig. 1). La vallée, large en cet endroit de près de $2,5 \mathrm{~km}$, est bordée de coteaux qui la dominent de $115 \mathrm{~m}$ à l'ouest (le Tertre) alors qu'à l'est, la pente est plus douce et moins élevée, de $45 \mathrm{~m}$ en moyenne.

À l'altitude de $85 \mathrm{~m}$ sur la terrasse la plus récente, la nécropole s'établit sur un léger microrelief, en dehors de la zone de divagation moderne du lit de l'Yonne (fig. 2). Le cours actuel de la rivière, distant d'une centaine de mètres, comportait de nombreux gués dus à des hauts-fonds gréseux. Étudiés au siècle dernier par A.-H. Robillard pour

et conservés sous l'autorité du Service régional de l'archéologie de Bourgogne, au dépôt de Passy (Yonne). Le générique de l'étude présentée dans notre article est le suivant: P. Chambon, les études des sépultures et la synthèse; J.-P. Delor, la fouille et le contexte régional; A. Augereau, le mobilier lithique; J. F. Gibaja Bao, l'étude fonctionnelle du matériel lithique; K. Meunier, la céramique; A. Thomas et P. Murail, l'anthropologie; D. Molez, le dessin lithique. Nos remerciements à Laurence Astruc pour sa relecture de la traduction de la contribution de J. F. Gibaja Bao. Les clichés des opérations de fouille qui accompagnent notre article ne comportent souvent ni orientation, ni échelle. Dans la mesure où ces informations figurent sur les plans, nous avons préféré ne pas intervenir sur les clichés. 


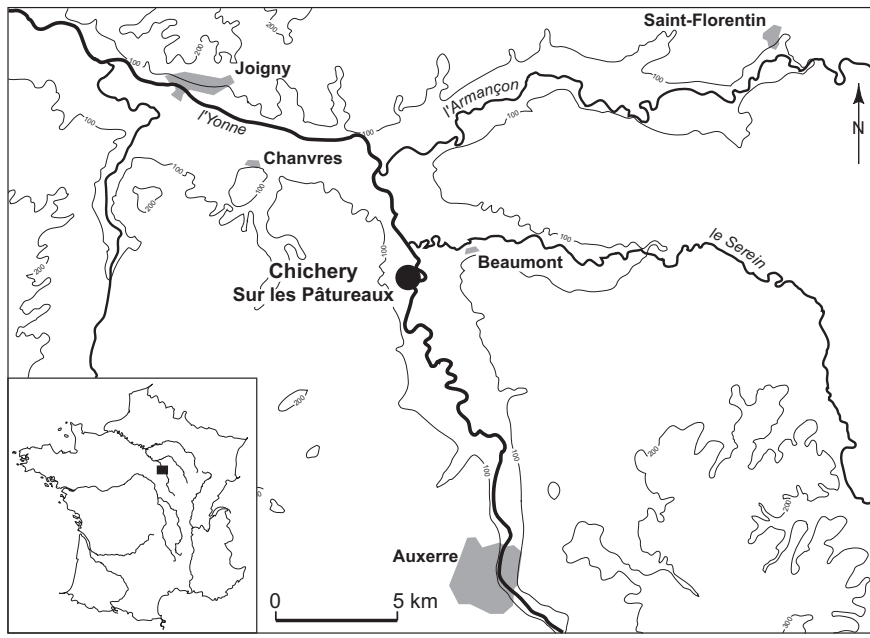

Fig. 1 - Sur les Pâtureaux à Chichery, dans la vallée de l'Yonne, à quelques centaines de mètres de la confluence avec le Serein, à $4 \mathrm{~km}$ au sud de la confluence avec l'Armançon (DAO: P. Pihuit, Inrap).

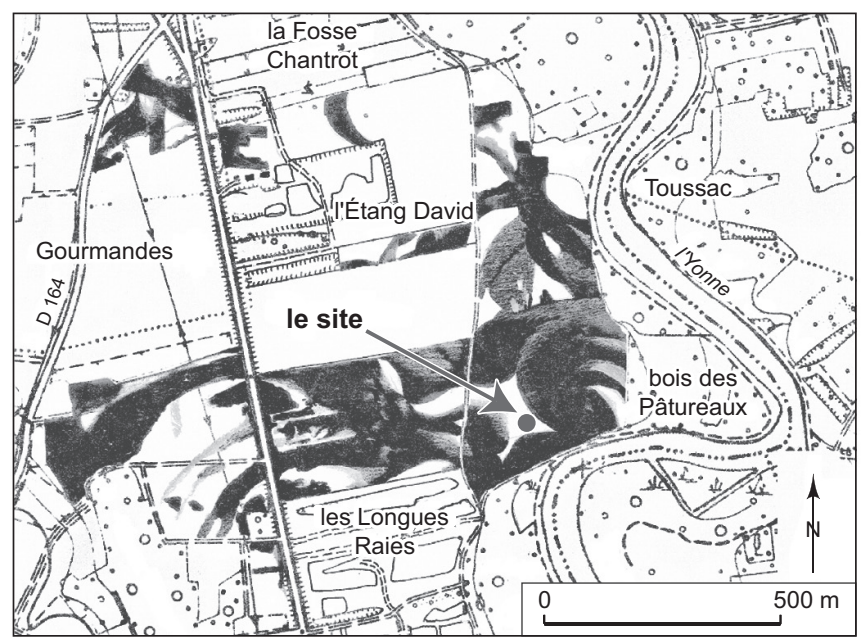

Fig. 2 - Le site de Sur les Pâtureaux à Chichery, sur un microrelief gravelosableux, au sein d'un environnement de paléochenaux. Les paléochenaux sont indiqués en noir; leur repérage a été réalisé sur les photographies aériennes, il reste donc incomplet (plan: J.-P. Delor).

les travaux préparatoires à la canalisation de l'Yonne ${ }^{7}$, les plans et les coupes effectués sur ces gués nous apprennent qu'à la borne 52, correspondant au Crot aux Moines, à quelque $800 \mathrm{~m}$ plus au nord, la profondeur de la rivière était de $0,50 \mathrm{~m}$ (coupe $\mathrm{n}^{\circ} 26$ relevée le 10 janvier 1836). Le climat

7. Archives départementales de l'Yonne, Auxerre: série S, en particulier 4S 188 - plans et croquis - entre Auxerre et Laroche, 1807 à 1835 . du Néolithique moyen étant assez voisin du nôtre, il y a fort à penser qu'en période de basses eaux, le passage d'une rive à l'autre de la rivière ne devait pas poser de difficultés particulières.

La plaine de Bassou est composée d'alluvions modernes qui reposent sur des nappes résiduelles d'alluvions anciennes de la basse terrasse de l'Yonne, légèrement en dessous de son lit majeur. La stratigraphie permet de distinguer:

- de $0 \mathrm{~m}$ à -0,26 m/-0,32 $\mathrm{m}$ : une couche de terre arable argilogravillonneuse, de couleur brun foncé, riche en matière organique et comportant une fraction limoneuse notable ainsi qu'une très forte proportion d'éléments de fort calibre (dragées). Cette couche correspond à la partie cultivée;

- de - 0,26 m à-0,36 m/-0,39 m: une couche semblable à la précédente mais nettement plus claire car moins chargée en matière organique; elle n'est atteinte par les labours qu'en quelques endroits;

- à partir de $-0,39 \mathrm{~m}$ : une couche de sédiments gravelosableux à sables grossiers comportant une forte proportion de calcaire récifal et kimméridgien qui excède $10 \mathrm{~m}$ d'épaisseur avec, parfois intercalés, des bancs de grès de 0,30-0,40 m d'épaisseur. C'est cette couche qui est massivement exploitée par de nombreuses entreprises de sables et graviers, tout au long de la vallée de l'Yonne. À l'est des sondages, la granulométrie est plus fine (sablon), alors qu'à l'ouest le sable est «sali» par des alluvions; de gros galets granitiques, quartzeux ainsi que des plaques de calcaire kimméridgien et de grès ferrugineux se mêlent aux sédiments.

Toutefois, la rivière lors de ses déplacements s'est creusée des chenaux qui peuvent localement atteindre plusieurs mètres de profondeur et qui se sont comblés de sédiments divers mais en surface desquels, au final, s'est déposée une couche de limons de couleur rougeâtre assez caractéristique. Ces paléochenaux se sont croisés jusqu'à une période relativement récente, aménageant des «plages» et des îlots graveleux sur lesquels des groupes humains ont pu s'installer préférentiellement.

\section{ENVIRONNEMENT ARCHÉOLOGIQUE DU SITE}

La vallée de l'Yonne est riche en sites archéologiques de l'époque néolithique. Ce foisonnement a été particulièrement bien reconnu pour deux raisons principales:

- depuis plus de quarante ans les sables et graviers extraits dans les carrières de granulats nécessitent la réalisation préalable du décapage total des parcelles. Ce sont 
ainsi plusieurs centaines d'hectares qui ont pu être étudiés, notamment à partir de 1994 dans le cadre de la convention «Archéologie et carrières dans l'Yonne» (Delor, 1995);

- la prospection aérienne (PA) est pratiquée, presque sans discontinuité, dans le bassin de l'Yonne depuis 1953, permettant d'enregistrer chaque année les vestiges de sites d'habitat, de nécropoles monumentales ou d'enceintes.

Pour la seule vallée de l'Yonne, dans un environnement immédiat et un rayon de moins de $3 \mathrm{~km}$, les sites suivants ont été repérés en prospection aérienne ou fouillés (fig. 3 et 4) : la nécropole de l'Étang David à Chichery, datée initialement du Néolithique ancien (fouilles C. Pellet; Pellet, 1978) ; l'enceinte à fossé interrompu de Sur les Pâtureaux à Chichery, qu'on peut rattacher, faute de fouilles, à un Néolithique moyen indéterminé (PA, sondages A. Galan en 1990); le village néolithique ancien de Bois des Marais à Chichery, avec une maison bien visible (PA) ; les enceintes à fossés interrompus et les habitats à maisons rondes de Toussac (PA) et du Crot aux Moines, tous les deux à Beaumont, et dont l'essentiel est associé au Chasséen (fouilles C. Pellet et A. Merlange, voir Pellet, Delor, 1981a et b) ; l'enceinte de rebord de plateau de la Petite Montagne à Bassou, non datée (PA) ; le village Rubané et VilleneuveSaint-Germain de Sur le Chemin de Cheny à Bonnard, avec ses quinze maisons identifiées (PA); le village néolithique ancien d'Écluse de Raveuse à Gurgy, et ses trois maisons (PA); l'enceinte à fossé interrompu de Néron à Gurgy, non datée mais sans doute du Néolithique moyen (PA); les enceintes à fossé interrompu du Nouzeau à Gurgy, dont la céramique évoque le Cerny (diagnostic F. Muller, 2004; fouille K. Meunier, 2005); le village VilleneuveSaint-Germain/Cerny de la Picardie à Gurgy, avec trois maisons (fouilles J.-J. Grizeaud, P. Nouvel et J.-P. Delor) ; la maison du Néolithique ancien de Toussac - camp militaire à Chemilly-sur-Yonne (PA).

Ces gisements constituent un environnement tout à fait exceptionnel qui se prolonge tout aussi densément le long de la vallée, au sud comme au nord, jusqu'au niveau de Joigny.

\section{HISTORIQUE DES DÉCOUVERTES}

Au début du mois d'août 1981, en effectuant des labours sensiblement plus profonds qu'à l'ordinaire, le propriétaire, Bernard Burat de Chichery, observait la remontée en surface, d'ossements, de cendres et de pierres brûlées. Il prévint la Direction des Antiquités et une autorisation de sauvetage urgent fut accordée à Jean-Louis Tainturier,

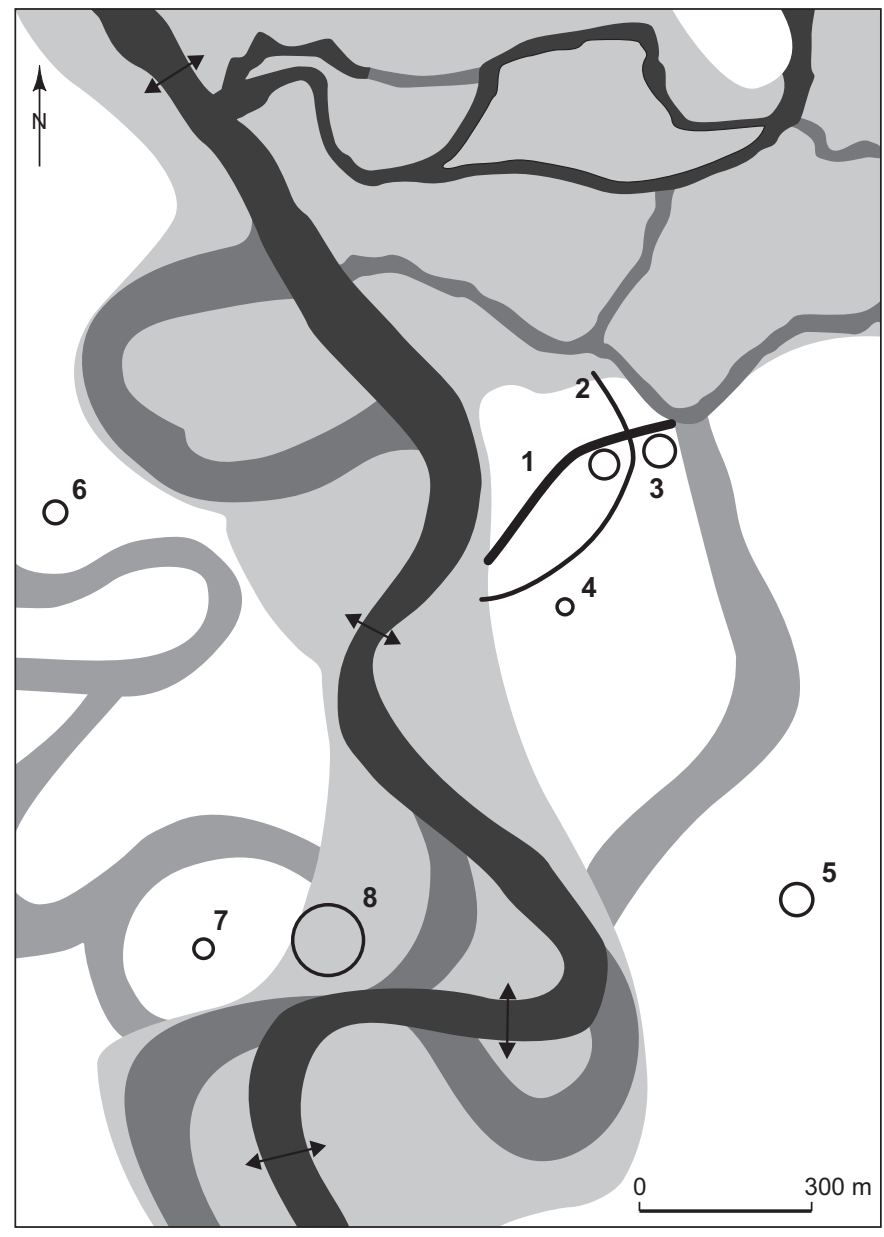

lit de I'Yonne et du Serein actuel lit fossile figurant sur la carte géologique divagation maximum des méandres récents

Fig. 3 - Implantations néolithiques à la confluence Serein-Yonne: 1, enceinte 1 du Crot aux Moines à Beaumont; 2, enceinte 2 de Toussac et du Crot aux Moines à Beaumont; 3, bâtiment circulaire néolithique moyen du Crot aux Moines à Beaumont; 4, bâtiment circulaire néolithique moyen de Toussac à Beaumont; 5, habitat néolithique ancien de Toussac - camp militaire à Chemilly-sur-Yonne; 6, nécropole du Néolithique moyen de l'Étang David à Chichery; 7, nécropole du Néolithique moyen de Sur les Pâtureaux à Chichery; 8 , enceinte de Sur les Pâtureaux à Chichery (DAO: J.-P. Delor).

seul archéologue disponible localement en cette période de vacances ${ }^{8}$. Il intervint aussitôt et mit au jour deux premières sépultures (S2 et S3) qu'il data du Néolithique moyen grâce

8. Le site porte le $\mathrm{n}^{\mathrm{o}}$ 89.105.002. Il figure sur la carte IGN 2619 Est Migennes. Les coordonnées Lambert II du point central sont $688.896 / 2324.109 / 85 \mathrm{~m}$. La parcelle fut cadastrée en 1958 sous la référence $\mathrm{ZD} 81$. Lors des diverses interventions, la parcelle concernée appartenait à M. Bernard Burat, domicilié à Chichery. 


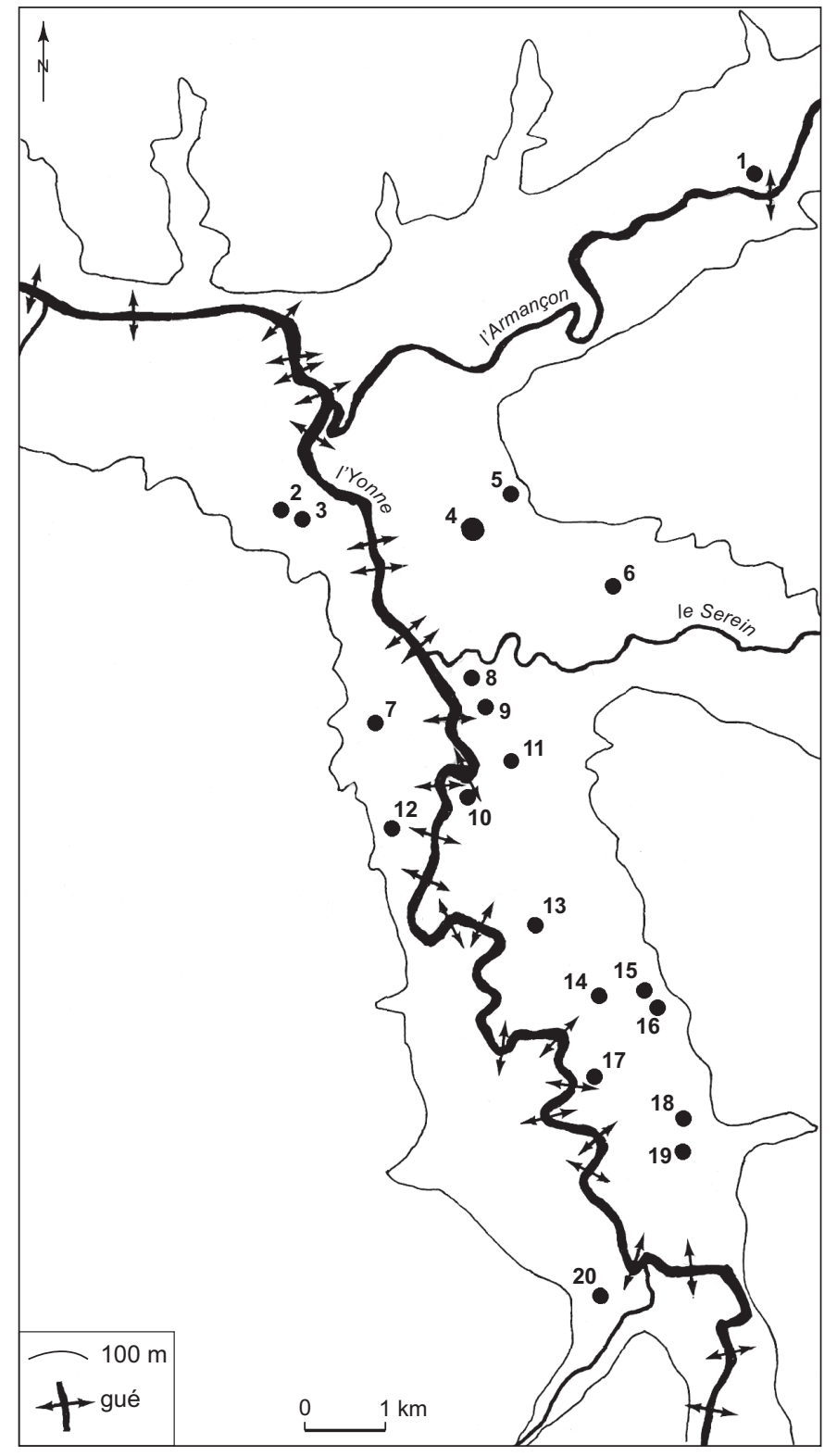

Fig. 4 - Les gués de l'Yonne avant sa canalisation entre Migennes et Auxerre. Les points indiquent les habitats néolithiques reconnus en prospection aérienne ou à la fouille: 1, le Bas d'Esnon à Esnon; 2, Sous les Ormes à Charmoy; 3, l'Orme à Charmoy; 4, Sur le Chemin de Cheny et le Saucy à Bonnard; 5, Mardelles et Groseilliers à Cheny; 6, le Crot aux Oies à Ormoy; 7, les Gourmandes à Bassou; 8, le Crot aux Moines à Beaumont; 9, Toussac à Beaumont; 10, Écluse de Raveuse à Gurgy;

11, Toussac à Chemilly-sur-Yonne; 12, Bois des Marais à Chichery; 13, Néron à Gurgy; 14, les Plantes du Mont I et II à Gurgy; 15, les Chaumes à Chemilly-sur-Yonne; 16, les Grands Champs à Gurgy;

17, la Motte à Gurgy; 18, les Champs d'Eau à Gurgy; 19, les Sept Arpents à Gurgy et Monéteau; 20, Macherin à Monéteau (DAO:J.-P. Delor). au mobilier lithique recueilli (Tainturier, 1981). À proximité, il fouilla encore une sépulture en position repliée (S1) et dégagea, sans la fouiller, une longue fosse remplie de cendres (F1), puis une autre, conique, remplie de galets (F2). Nous disposons d'un rapport de fouille photocopié ${ }^{9}$.

Une seconde campagne de sondages eut lieu en 1983 par J.-P. Delor pour tenter de déterminer l'étendue de la nécropole, rechercher des indices pouvant préciser sa datation et trouver des éléments d'appréciation quant à l'éventuelle mise en place d'une fouille programmée. Les sondages furent réalisés au nord-ouest des découvertes précédentes et permirent la fouille de trois nouvelles sépultures (S4-S6) et d'une nouvelle fosse (F3) contenant de nombreuses pierres (Delor, 1983).

En 1984, une opération de sauvetage programmé fut autorisée. Le décapage, effectué avec un engin de travaux publics, concerna $600 \mathrm{~m}^{2}$ de terrain, au nord et à l'ouest, tout en reprenant le secteur des interventions antérieures. Sept nouvelles sépultures furent fouillées (S7-S13), ainsi que vingt-quatre fosses, trous de poteaux et tronçons de fossés divers (Delor, 1984). Une distribution spatiale des sépultures en deux alignements, dont un enceint dans une éventuelle construction sur poteaux, avait alors été avancée (fig. 5).

Pour ces deux campagnes nous disposons de plusieurs centaines de photographies noir et blanc et de diapositives, ainsi que de toute la documentation de fouilles nécessaires à la rédaction des rapports: relevés, notes, plans et coupes.

Une quatrième intervention fut programmée en 1990 et conduite par Anne Galan. Le rapport de l'opération fait défaut, mais quelques relevés en notre possession montrent qu'au moins deux grandes fosses furent fouillées plus au nord-est, là où la prospection aérienne avait révélé diverses structures fossoyées à l'intérieur d'une enceinte palissadée (coordonnées Lambert II : 689.014/2 324,100) (fig. 6).

Durant les deux premières années de la fouille, le décapage a été réalisé manuellement depuis le sommet de la terre arable. Cet exercice, imposé par le manque de moyens alloués à l'opération, a toutefois permis de noter précisément le niveau d'apparition des structures archéologiques. En outre, le voisinage immédiat du sol actuel a également conduit à fixer la profondeur des structures depuis cette surface. En 1984, le décapage mécanique change cette

9. Nous remercions chaleureusement $\mathbf{M}^{\text {me }}$ Tainturier pour nous avoir confié toute la documentation en sa possession, ainsi que l'ensemble du matériel mis au jour lors des fouilles de son mari. A sa demande, ce mobilier est désormais réuni à celui des fouilles de J.-P. Delor et déposé au sein du dépôt État de Passy. 


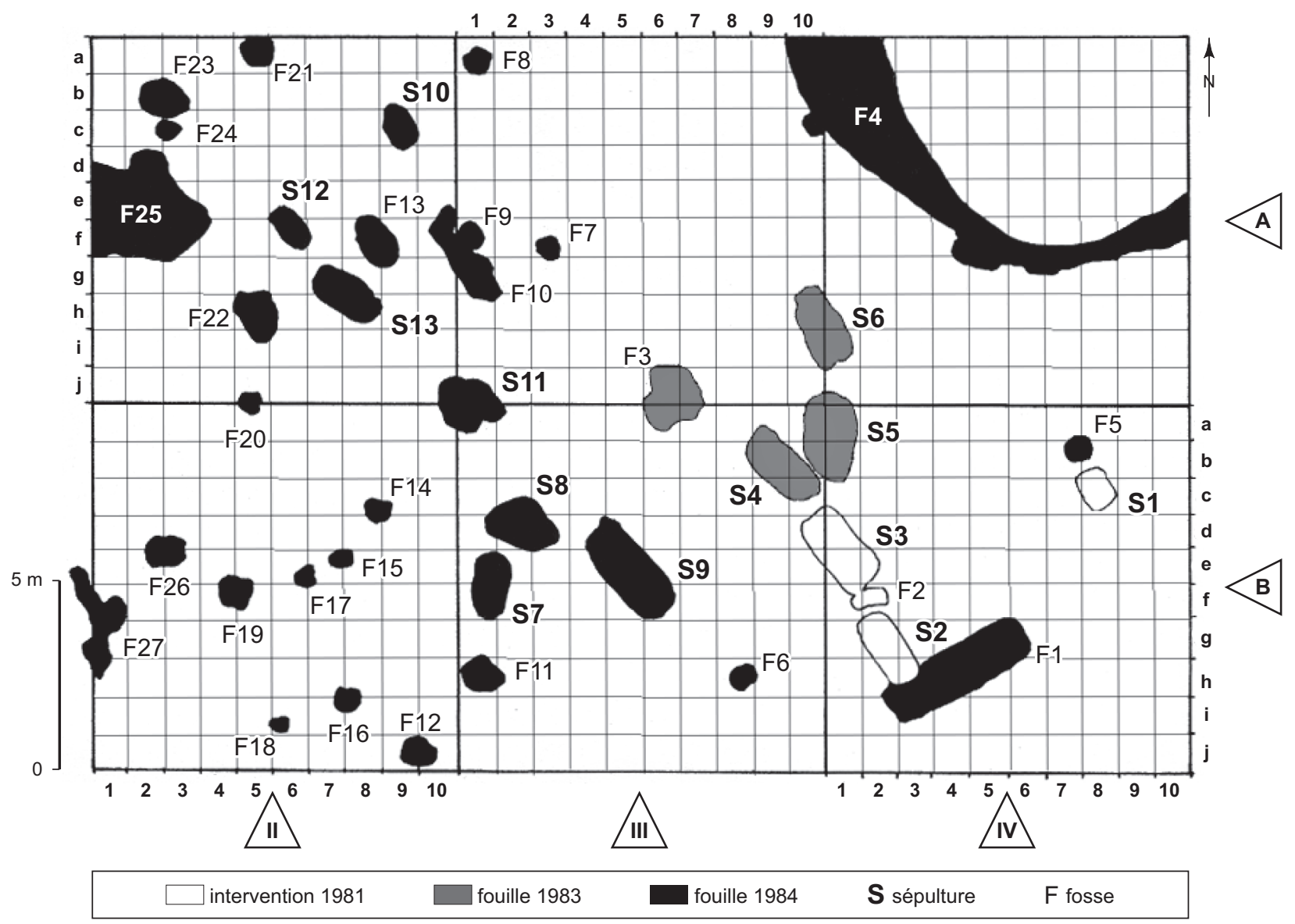

Fig. 5 - Relevé des structures découvertes et fouillées de 1981 à 1984 (DAO: J.-P. Delor).

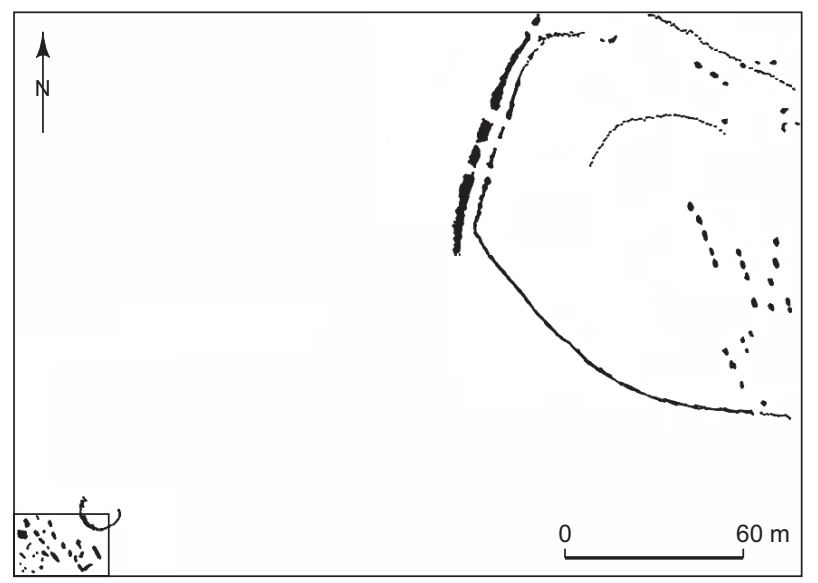

Fig. 6 - Situation des fouilles 1981-1984 (en bas à gauche) par rapport à l'enceinte contenant les fosses sondées en 1990 (DAO: J.-P. Delor).

donne et c'est la surface décapée qui sert de référence, comme c'est le cas désormais sur les grands décapages. Dans les descriptions qui suivent, nous avons conservé les deux systèmes de référence, d'une part pour ne pas unifier arbitrairement les informations et, d'autre part, parce que le premier système a l'avantage d'être plus précis, car il ne dépend ni de l'habileté du pelleteur, ni du soin de l'archéologue qui le guide. 


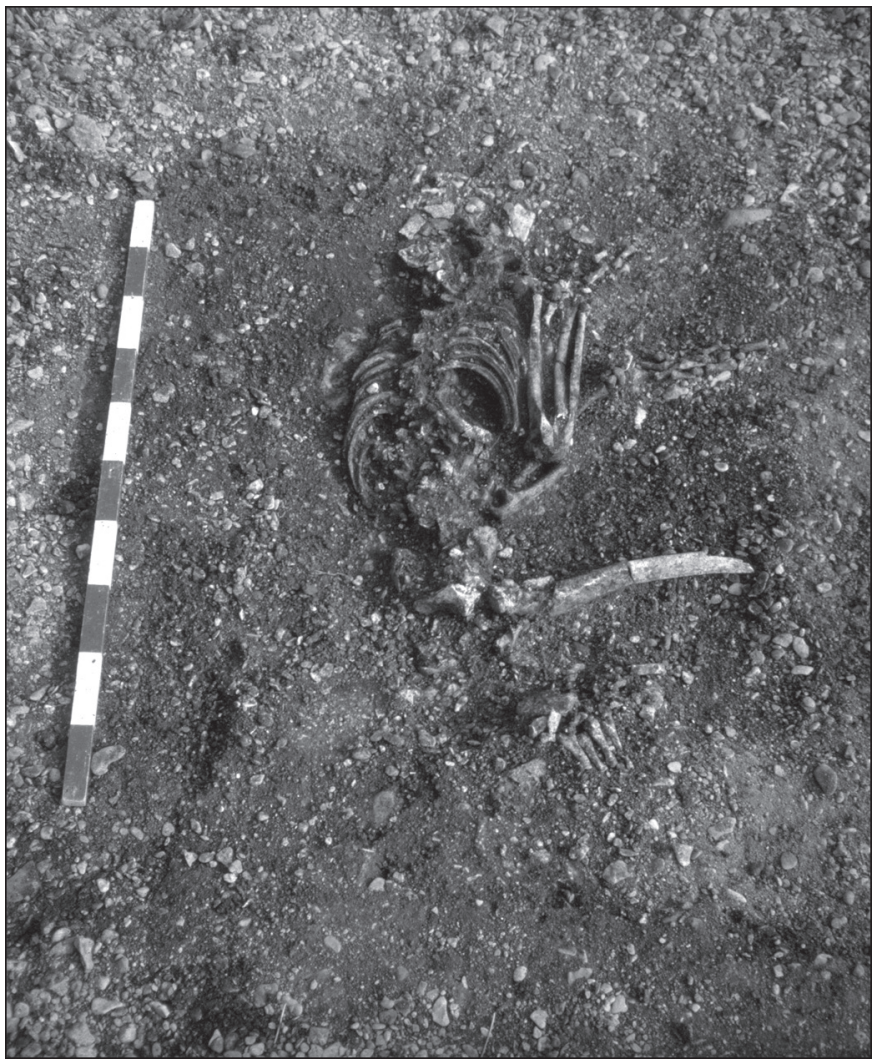

Fig. 7 - La sépulture 1 après dégagement du squelette; l’absence de fosse et le caractère partiel du squelette sont dus à sa situation à la base de la semelle de labours (cliché: J-L. Tainturier).

\section{LES STRUCTURES : DESGRIPTION ET INTERPRÉTATION}

\section{SÉPULTURE 1}

La sépulture $1^{10}$ est la plus originale de la partie fouillée de la nécropole. C'est en effet la seule qui comprenne un sujet en position fléchie sur le côté (fig. 7 et 8). Très superficielle sous les labours, elle ne mesurait plus que $0,15 \mathrm{~m}$ de profondeur en son point le plus bas (soit tout de

10. Chaque sépulture pouvant être considérée comme un microgisement, nous les présentons séparément, de la description à l'interprétation. L'analyse de la structure reprend les principes de l'analyse taphonomique, initiée par F. Poplin (1975), appliquée aux sépultures individuelles par D. Mordant (1987), et systématisée par H. Duday (1995). Les caractéristiques principales des individus et du mobilier associé sont indiquées. L'analyse systématique des éléments constitutifs des sépultures, ainsi que les méthodes mises en œuvre pour leur étude, sont développées dans la troisième partie («Les pratiques funéraires, trait par trait»).

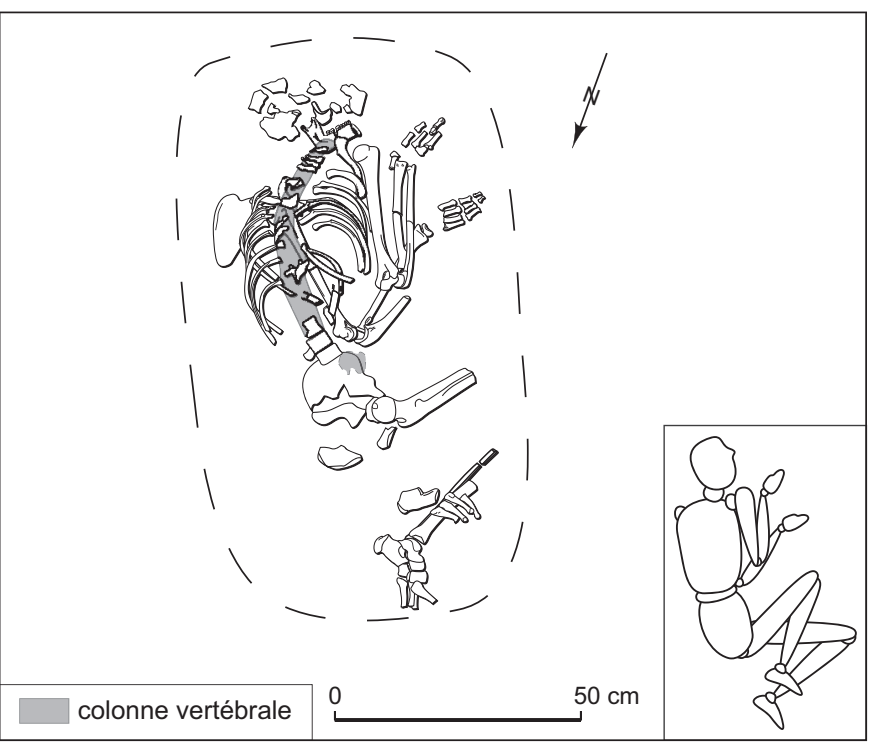

Fig. 8 - Plan de la sépulture 1 réalisé d'après les photographies redressées à partir du relevé figuré dans le rapport de J.-L. Tainturier (1981). La moitié distale du fémur droit, clairement "replacé» sur la photographie, n'a pas été représentée. Le contour que nous indiquons en pointillé ne correspond qu'à une partie du fond de la fosse telle que la fouille a pu la dégager; en dépit de sa faible signification, il correspond aux contours de la structure sur le plan général. En encadré, restitution de la position du sujet (relevé: P. Chambon, CNRS).

même à plus de 0,40 m sous le sol actuel). Elle a été creusée dans le sable encaissant, sans aménagement particulier, et le fond se présente sous la forme d'une cuvette très plate. Les limites de la fosse n'ont pas été reconnues. Le contour figuré correspond vraisemblablement à une partie du fond, sans aucun retour de paroi ; les genoux ne pouvaient qu'être à l'extérieur de l'ovale représenté. Nous n'avons pas d'information sur le comblement de la sépulture, sans doute limoneux, toutefois la faible profondeur n'aurait pas autorisé la reconnaissance de subdivision.

Le sujet est orienté sud-nord, le regard tourné vers le sud-ouest. Il repose en position fléchie, le tronc pour moitié sur le côté gauche, pour moitié tourné contre le sol. Tous les membres sont fléchis. L'épaule droite est haussée, les bras sont contre le thorax, le coude gauche fléchi à $45^{\circ}$, le droit hyperfléchi. La main gauche se trouve devant le thorax, la main droite en avant de l'épaule. La situation des membres inférieurs, incomplets, est plus imprécise: les hanches sont fléchies autour de $90^{\circ}$, les genoux de $30-45^{\circ}$. Les pieds ne sont pas contraints. 


\section{CONSERVATION ET PERTURBATIONS}

L'état général des ossements est plutôt bon. La surface des os est peu altérée, les extrémités des os longs sont présentes au même titre que les diaphyses, et des os courts sont visibles. Cependant, on ne distingue pas de corps vertébraux sur les photographies, même s'ils figurent sur le plan schématique.

Si l'érosion chimique est peu importante, on ne peut en dire autant des destructions mécaniques. La sépulture a souffert du passage de la charrue: les ossements sont fragmentés, une partie importante d'entre eux a été récupérée dans la semelle de labours et il subsiste des manques. Parmi les plus facilement identifiables, il faut citer la moitié droite de la tête osseuse, la scapula droite, la moitié distale de l'ulna gauche, l'essentiel du bassin et tout le membre inférieur droit (seul le pied est partiellement présent), et la moitié médiane du gauche. Les déficits les plus importants concernent les parties les plus épaisses du squelette, au vu de sa position (principalement la tête osseuse, plus ponctuellement le bassin et les membres inférieurs). Le décapage manuel de la zone fouillée en 1981 assure que les labours sont bien à l'origine de ces destructions.

L'érosion de la tombe, ainsi que les lacunes de la documentation ne permettent pas d'exclure d'éventuelles bioturbations de la tombe. Toutefois, les photographies ne montrent aucune aberration anatomique. Nous considérons donc que la position des vestiges correspond globalement à l'évolution du cadavre dans la sépulture.

\section{CONNEXIONS ET DISLOCATIONS}

La discussion taphonomique est fortement grevée, d'un côté par l'érosion de la sépulture, d'un autre côté par la documentation.

Il est impossible de se prononcer sur le squelette axial. La disposition des pièces visibles n'implique aucune dislocation depuis le bloc craniofacial jusqu'au sacrum. Les côtes se sont affaissées: le tronc étant largement tourné contre le sol, elles apparaissent logiquement par leur face inférieure. Aucune pièce n'est sortie ici de manière convaincante $\mathrm{du}$ volume initial du cadavre; quelques fragments du bloc craniofacial sont dispersés, mais cette dispersion paraît davantage l'œuvre de la charrue, voire un effet de fouille.

La scapula gauche s'est effondrée sur le sol, en vue antérieure. Le coude est disloqué: l'ulna est décrochée de la trochlée humérale et elle diverge du radius. Celui-ci est dans sa position initiale, au contact d'un carpien. De la main gauche, on distingue quatre métacarpiens en connexion avec leurs phalanges proximales, suivies par deux phalanges moyennes. La scapula droite manque, toutefois la clavicule est "verticalisée", parallèlement à l'humérus. L'ulna est décrochée mais reste au contact de l'humérus. L'avant-bras est en connexion, quelques ossements de la main paraissent déplacés.

L'os coxal droit est réduit à une portion d'aile de l'ilium, sa position en arrière de l'os coxal gauche montre une relation côté droit-côté gauche différente de celle qui prévaut pour le haut du corps. Si cette position n'est pas liée à une perturbation, elle traduit l'effondrement de l'os coxal vers l'arrière, hors du volume initial. La tête du fémur gauche est encore logée dans l'acétabulum. Le genou gauche est détruit, toutefois les axes respectifs du fémur et du tibia convergent bien au-delà de son emplacement estimé: le fémur semble avoir pivoté vers le sud. Le pied est globalement connecté, à l'exception du premier métatarsien visible qui diverge; la photographie ne permet pas une analyse détaillée. Du côté droit, on n'aperçoit que le calcanéus et quatre métatarsiens, dont les liens sont relâchés par-dessus le tibia gauche.

\section{MILIEU DE DÉCOMPOSITION ET TYPE DE STRUCTURE}

L'absence d'une partie des restes osseux ne permet pas d'être catégorique, toutefois le bilan ostéo-articulaire est univoque. Des ossements sont sortis du volume initial du cadavre: la scapula gauche effondrée sur le sol, la clavicule droite «verticalisée», sans doute l'os coxal droit, qui apparaît en arrière du gauche, et le métatarse droit affaissé contre le tibia gauche. Inversement, nous n'avons pas pu distinguer d'ossement en situation de déséquilibre: la main gauche affiche une belle cohérence, mais elle repose à plat sur le fond; seul le pied gauche aurait pu révéler telle situation, malheureusement les documents sont trop peu précis.

Aucun élément ne permet d'envisager la structure sépulcrale. La totalité du fond de fosse n'a pas pu être reconnue. Le squelette n'est pas contraint par son contenant: seules les parties manquantes pouvaient éventuellement jouxter la paroi de la tombe, c'est-à-dire les orteils et l'un ou l'autre des genoux. En définitive, nous écarterons le simple linceul autour du corps, mais toutes les solutions restent possibles, de la fosse à couverture jusqu'au caveau bâti. 


\section{L'INDIVIDU}

Conservation osseuse: la qualité osseuse du squelette 1 est bonne mais présente un taux de fragmentation relativement élevé. La tête osseuse n’est représentée que par un fragment du frontal et de l'occipital gauche; la mandibule est partiellement conservée. Les extrémités des os longs sont rarement présentes. Les os coxaux, fortement fragmentés, ont pu être partiellement remontés.

Âge: la maturation complète de l'extrémité sternale de la clavicule de cet individu indique un âge supérieur à 25 ans, confirmant le diagnostic adulte fait en 1981 (Tainturier, 1981). L'estimation de l'âge à partir de la surface sacroiliaque permet de préciser un âge supérieur à 30 ans.

Sexe: le mauvais état des os coxaux ne permet pas l'observation des trois critères requis au minimum par la méthode morphoscopique. Toutefois, le remontage partiel de ces fragments nous a permis de réaliser quatre mesures et d'en estimer une cinquième (IIMT). Considéré dans sa fourchette maximale, le résultat de la DSP reste celui d'un individu masculin. Ce résultat est cohérent avec la forme de l'arc composé des os coxaux et les critères crâniens observés.

Stature: uniquement calculée à partir des os de l'avantbras, la stature de l'individu 1 est comprise entre 1,64 m et 1,69 m, légèrement supérieure aux estimations faites en 1981 (Tainturier, 1981).

État buccodentaire: les dix-neuf dents conservées (isolées pour la moitié) montrent une usure très avancée. L'abrasion des cuspides des dents antérieures est importante, allant jusqu'à la confluence des îlots dentinaires. Les quatre premières molaires présentent une usure totale de la hauteur coronale; l'usure des deuxièmes et troisièmes molaires supérieures et inférieures n'est qu'à peine moins prononcée. Nous notons la présence de deux caries de sévérité moyenne sur le collet des deuxième et troisième molaires supérieures gauches, au niveau de leur face de contact. Les neuf dents inférieures observées en place sur la mandibule laissent apparaître la partie supérieure de leur racine, témoignant d'une légère parodontose. Enfin, un dépôt modéré de tartre est observé sur chaque dent, généralement sur leur face vestibulaire et systématiquement au niveau de leur collet.

Indicateurs de stress biologique: une hypoplasie linéaire de l'émail dentaire a été observée sur les trois canines présentes. De faible sévérité, cette hypoplasie se situe dans chaque cas au niveau du tiers moyen de la couronne. L'importante usure des autres dents conservées limite l'observation d'éventuelles autres atteintes du même type.
Pathologies: l'individu 1 présente plusieurs atteintes pathologiques, principalement rachidiennes. Les vertèbres présentent de nombreuses excroissances osseuses au-delà des plateaux vertébraux. Ces ostéophytes peuvent prendre l'aspect d'un bec de 2-5 mm se développant soit vers le bas (à partir du bord inférieur), soit vers le haut (à partir du bord supérieur), notamment sur les vertèbres cervicales (VC) et lombaires (VL). La hauteur des corps vertébraux est conservée bien que la surface des plateaux soit irrégulière (une faible érosion localisée est parfois visible) et criblée de foramens, particulièrement visible sur les VC. Les VC 3 et 4 montrent une ankylose intra-articulaire des corps et des processus postérieurs. Les vertèbres thoraciques (VT) montrent une déformation des surfaces articulaires. La $8^{\text {e }}$ VT présente une ankylose marginale de la surface articulaire costale gauche. Ces observations (associées à celles sur le calcanéus, la patella et l'ulna) excluent la maladie hyperostosique. Cette atteinte arthrosique pourrait être liée à un âge avancé de l'individu.

Le radius gauche présente une dépression localisée de forme ovalaire sur la face postéromédiale de la diaphyse, à $2,5 \mathrm{~cm}$ sous la tubérosité radiale. Longue de $18 \mathrm{~mm}$ et large de $9 \mathrm{~mm}$, la perte de substance est superficielle, ne dépassant pas la surface de la substance compacte. La surface d'érosion est irrégulière et criblée de foramens. Le contour présente un modelage osseux irrégulier sur le bord médial, s'étendant vers l'inférieur sur $10 \mathrm{~mm}$ au-delà de la dépression. Cette observation est unilatérale.

Le tibia droit présente une déformation axiale et volumique de forme angulaire, localisée à mi-hauteur de la diaphyse (prend l'aspect d'un pic au niveau de la crête antérieure développé vers l'antérieur). La surface est régulière, la texture est homogène. Cette déformation est associée à un bourrelet osseux, s'apparentant à un cal, d'aspect régulier et peu volumineux mais nettement identifiable au touché. La radiographie ${ }^{11}$ ne semble décrire aucune trace de fracture.

\section{SÉPULTURE 2}

La sépulture 2 n'est pas totalement inédite, une photographie accompagnant la notice sur le site a été publiée dans les informations archéologiques de Gallia Préhistoire (Thévenot, 1982). Elle apparaissait au décapage comme une fosse allongée, orientée nord-ouest/sud-est,

11. Les radiographies ont été réalisées au Laboratoire d'anthropologie des populations du passé (LAPP) de l'UMR 5199, à Talence. 


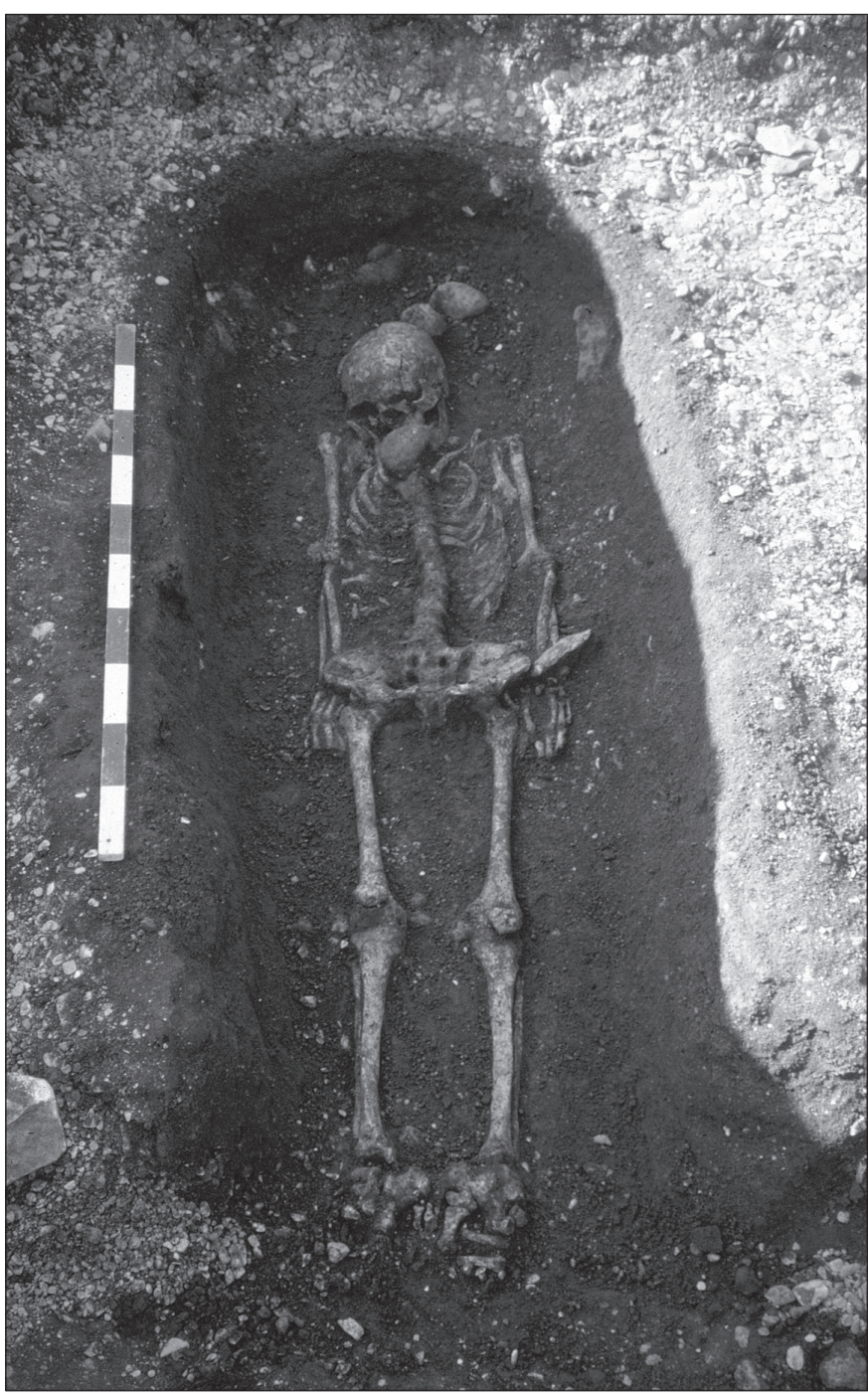

Fig. 9 - Vue générale de la sépulture 2 après dégagement du squelette. La position du galet de granite, contre la poitrine, est-elle fortuite? Noter la position du pic-ciseau sur l'extrémité de l'avant-bras gauche (cliché:J.-L. Tainturier).

de forme rectangulaire, aux angles arrondis. Elle mesurait $2,00 \mathrm{~m} \times 1,05 \mathrm{~m}$ et atteignait $0,48 \mathrm{~m}$ de profondeur sous la terre végétale. Son remplissage est constitué de terre fine de couleur brune et le squelette repose au centre de l'excavation qui paraît à peine suffisante pour le recevoir. Nous ne disposons d'aucune coupe mais huit photographies permettent d'apprécier sa forme (fig. 9). Les bords en semblent presque verticaux et le point le plus bas paraît se situer sous les jambes.

Pour cette sépulture comme pour les suivantes, une série de questions se pose. La fosse est creusée dans le gravier

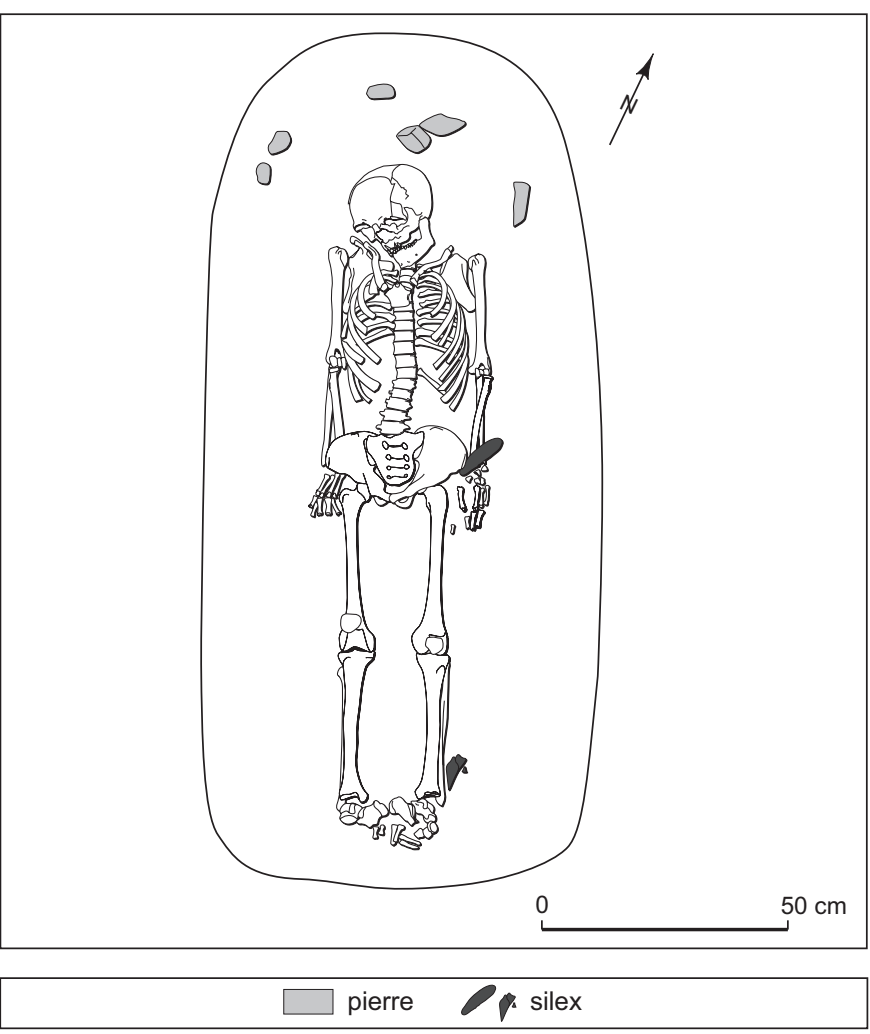

Fig. 10 - Plan de la sépulture 2 réalisé d'après les photographies redressées. Les clichés sont très clairs et précis pour le tronc et la tête, plus lointains pour les membres. Nous l'avons mis à l'échelle à partir du relevé figuré dans le rapport de fouille de J.-L. Tainturier (1981). L'absence d'une partie des côtes tient à leur non-dégagement sur les vues dont nous disposons (relevé: P. Chambon, CNRS).

dont près de $1 \mathrm{~m}^{3}$ a été extrait: où a-t-il été déposé ? Qu'est-il devenu puisque la sépulture n’a été comblée qu'avec de la terre arable? D'où provient cette terre humifère souvent riche en silex et tessons? Existe-t-il un habitat tout proche? Pourquoi avoir opéré cette permutation de matériaux?

La tombe comprend un sujet adulte en position étendue sur le dos, la tête au nord-ouest et les pieds au sud-est, situé dans l'axe et à la base d'une fosse de $2 \mathrm{~m}$ de long et $1,05 \mathrm{~m}$ de large, pour $0,50 \mathrm{~m}$ de profondeur sous la terre arable. Un galet de granite, oblong et long de 9,3 cm, a été retrouvé sur la poitrine, contre le menton. Six petites pierres étaient contenues à la base du remplissage, à l'extrémité nord de la fosse, dont une directement en contact avec le bloc craniofacial.

La tête de l'inhumé est légèrement redressée et basculée vers l'épaule droite, la bouche est fermée. Les épaules sont haussées. Les membres supérieurs sont étendus et plaqués contre le tronc. La main gauche est de chant, la main droite 
en pronation, sans doute légèrement fléchie. Les membres inférieurs sont étendus parallèlement.

La position des épaules dénote une compression à ce niveau. Les bords latéraux des membres inférieurs définissent deux droites parallèles, mais qui ne sont prolongées ni au niveau du bassin, ni au niveau des membres supérieurs.

Le mobilier associé comprend cinq pièces en silex: un pic-ciseau sur les os de l'avant-bras gauche, trois armatures contre la jambe gauche, à proximité de la cheville, et un racloir (fig. 10).

\section{CONSERVATION ET PERTURBATIONS}

Les photographies révèlent un squelette bien conservé. Tant la substance compacte que la substance spongieuse de l'os sont présentes. Les os longs sont munis de leurs épiphyses, la colonne vertébrale exhibe ses corps vertébraux, le manubrium se dessine clairement sur la vue du tronc.

Aucune perturbation n'a été notée dans le rapport de fouille. On ne distingue rien de tel sur les photographies et la situation des vestiges ne révèle pas d'aberration anatomique. L'absence de pièces petites ou fragiles, comme les phalanges distales ou le corps du sternum, témoigne d'un problème de conservation ou constitue des effets de fouille. On peut estimer que la position des vestiges, telle qu'elle apparaît sur les photographies, résulte de la décomposition du corps au sein de la sépulture.

\section{CONNEXIONS ET DISLOCATIONS}

Le bloc craniofacial est quelque peu écrasé, mais la fragmentation ne se traduit pas par un éclatement. Il paraît s'être tassé sous l'effet d'une pression importante exercée du haut vers le bas, et penche vers l'épaule droite. La mandibule semble encore en connexion. Les vertèbres cervicales ne sont pas visibles. L'enchaînement des vertèbres, en vue antérieure, est ensuite respecté jusqu'au sacrum. Les côtes se sont logiquement affaissées dans le volume thoracique, toutefois sur les vues on ne distingue ni la côte 1 gauche, ni la partie inférieure du thorax. Le manubrium s'est également effondré dans le volume thoracique et repose en pendage entre les vertèbres et les côtes droites.

Les liens sont distendus au niveau des épaules, mais sans dislocation incontestable. La situation des membres supérieurs est la plus difficile à percevoir sur les vues (ils sont partiels ou au second plan). L'humérus gauche apparaît en face antérieure, le droit en face antérieure ou plus vraisemblablement antérolatérale (dans ce cas en déséquilibre).
Le seul constat possible concernant les coudes est qu'ils ne sont pas nettement disjoints. Les ossements des mains respectent la cohérence anatomique, mais la relation métacarpiens-phalanges n'est jamais stricte et, du côté gauche, le métacarpien 1 est clairement disloqué: son extrémité proximale s'écarte de quelques centimètres du poignet et son extrémité distale est décalée par rapport à ce qui semble être la phalange proximale 1 .

Le bassin est totalement ouvert. On ne peut juger de la situation des hanches, mais les fémurs sont demeurés en face antérieure, les patellas sur leurs extrémités distales. La droite a migré vers la diaphyse. Les tibias prolongent logiquement les fémurs, mais ils ont légèrement pivoté et se montrent sous leur face antéromédiale. Les pieds sont disloqués, une part de cette dislocation est sans doute un effet de fouille. La situation du tarse postérieur paraît plus assurée, révélant la complète rupture des chevilles: les talus, en vue antéromédiale, s'écartent de l'extrémité distale des tibias; les calcanéus, également disjoints, sont en vue médiale.

\section{MILIEU DE DÉCOMPOSITION ET TYPE DE STRUCTURE}

Les dislocations militant pour une décomposition en espace vide sont peu nombreuses. Les clavicules sont peutêtre sorties du volume initial du cadavre. Plus indiscutables sont les cas du métacarpien 1 gauche, des os coxaux et des os du tarse postérieur. Inversement, les arguments en faveur de l'espace colmaté sont presque inexistants: le bloc craniofacial est en déséquilibre, l'humérus droit l'est peutêtre aussi. La situation des radius, à cheval sur les ulnas, est ambiguë, il paraît en tout cas difficile de les utiliser dans l'argumentation. Enfin, les patellas, bien qu'encore liées aux fémurs, ne paraissent pas en situation instable. La balance nous paraît davantage pencher du côté de l'espace vide.

Peut-on, dès lors, proposer une solution à la position de la tête? Son déséquilibre est indissociable de l'écrasement de la face. Le comblement final de la sépulture est sans doute à l'origine de cet écrasement: avant cet événement la tête devait être plus verticale, comme en témoigne la position de la mandibule; on peut suggérer que la chute de la couverture ait provoqué la déviation de la tête vers l'épaule droite. Quant à l'humérus, sa position latérale peut expliquer un éventuel déséquilibre, les membres supérieurs étant contraints.

Pour approcher le dispositif sépulcral, nous disposons d'un effet de compression sur les épaules, de la position «ramassée» des membres supérieurs et de vagues effets de 
paroi sur les membres inférieurs. On peut sans doute écarter l'hypothèse d'une enveloppe souple (linceul), au moins comme solution unique: la seule contrainte se trouve au niveau des épaules et l'enveloppe souple s'accommode mal du parallélisme des membres inférieurs. Toutefois, si on envisage une structure rigide, où faut-il mettre ses parois? La compression et l'effet de paroi suggèrent un dispositif au plus près du corps, mais les membres supérieurs se trouvent à l'extérieur des limites définies par les membres inférieurs. Ces effets de parois sont-ils trop vagues et donc non significatifs? Leur prolongement vers le haut du corps nous conduit jusqu'aux articulations scapulo-humérales, où se trouve précisément la contrainte. Pour que ni la contrainte ni l'effet de paroi ne s'appliquent à la main droite et au membre supérieur gauche, on peut suggérer soit que sa hauteur est irrégulière, soit que cette paroi n'est pas partout sensible: on songe à une sorte de civière, c'est-à-dire un contenant à bord rigide et fond souple, le bord ne constituant une véritable paroi que dans certaines situations, comme le transport. Il s'agit là, avouons-le, d'une hypothèse opportuniste, donc insatisfaisante. Cependant, on doit insister sur l'idée d'un contenant au plus près du corps, qui ne constitue pas une limite stricte pour le sujet. Par conséquent, ce contenant n'est pas clos et il faut envisager que la couverture soit assurée par un second dispositif indépendant du premier. Nous n'avons aucun moyen de préciser lequel, il peut s'agir d'un simple couvercle par-dessus la fosse ou bien d'un caveau aménagé à même la fosse (les dimensions de celle-ci l'autorisent). C'est ce second dispositif qui a permis la décomposition en espace vide.

\section{L'INDIVIDU}

Conservation osseuse: la conservation du squelette 2 est excellente, tant du point de vue de la qualité de l'os que de sa représentation.

Âge: la maturation complète de la clavicule et de la crête iliaque situe d'emblée cet individu parmi ceux âgés de plus de 25 ans. L'analyse de sa surface sacro-pelvienne permet de préciser cet âge en lui attribuant une limite supérieure à 40 ans.

Sexe: l'observation des trois premiers critères de la méthode morphoscopique (région préauriculaire, grande incisure ischiatique et arc composé) nous conduit au même diagnostic que celui réalisé en 1981 d'un sujet masculin (Tainturier, 1981). Ces résultats, confirmés par la DSP, sont cohérents avec les caractères crâniens masculins que nous avons constatés.
Stature: la mesure des fémurs de cet individu nous a permis d'estimer sa stature à $1,57 \mathrm{~m}$ environ, proche de l'estimation initiale de 1,60 m (Tainturier, 1981).

État buccodentaire: l'ensemble des dents a été observé in situ (mandibule et os maxillaire conservés). À l'exclusion des quatre troisièmes molaires, l'ensemble des dents présente une usure relativement marquée bien qu'un pourtour d'émail soit systématiquement conservé. Les premières molaires droites, dont la moitié de la hauteur coronale est abrasée, sont les plus sévèrement atteintes. L'usure des quatre premières molaires semble en effet s'être opérée de façon asymétrique, à la faveur du côté gauche. L'individu ne souffre d'aucune lésion carieuse ou de maladie parodontale. Les dépôts de tartre sont légers et non systématiques. Ils n'atteignent que la face linguale des couronnes inférieures et la face vestibulaire des couronnes supérieures, à l'exception des incisives.

Indicateurs de stress biologique: l'hypoplasie linéaire de l'émail dentaire n'est clairement attestée que sur la canine supérieure gauche. Bien que de faible intensité, deux lignes sont clairement distinctes sur le tiers moyen et le tiers occlusal de la couronne.

\section{LE MOBILIER}

1) Armature de flèche perçante losangique sur éclat mince: retouche bifaciale oblique alternante sur les trois quarts de la pièce, uniquement directe sur l'apex; dimensions: $55 \mathrm{~mm}$ x $21 \mathrm{~mm}$ x $4 \mathrm{~mm}$ (fig. 11, $\mathrm{n}^{\circ}$ 1).

2) Armature de flèche tranchante trapézoïdale sur éclat mince: retouche bifaciale oblique à rasante, néanmoins plus abrupte sur le bord droit de la face inférieure; à partir des deux bords, c'est d'abord la face inférieure qui a été retouchée, puis la face supérieure; dimensions: $16 \mathrm{~mm}$ x 15 mm x 2 mm (fig. 11, no 2).

3) Armature de flèche perçante triangulaire sur éclat mince: l'attribution de cette pièce à la catégorie des armatures de flèche perçante repose sur son orientation dans la tombe, similaire aux deux autres armatures dont l'emplacement de la partie active ne peut être mis en doute; en effet, pour celle-ci, une incertitude subsiste compte tenu que l'apex n'est pas retouché sur les deux faces; la concavité de la partie opposée à la pointe n'est pas non plus un argument: il existe des armatures tranchantes à tranchant concave; la retouche est bifaciale oblique sur le bord droit, inverse sur le bord gauche; dimensions: $35 \mathrm{~mm}$ x $17 \mathrm{~mm}$ x 4 mm (fig. 11, no 3). 

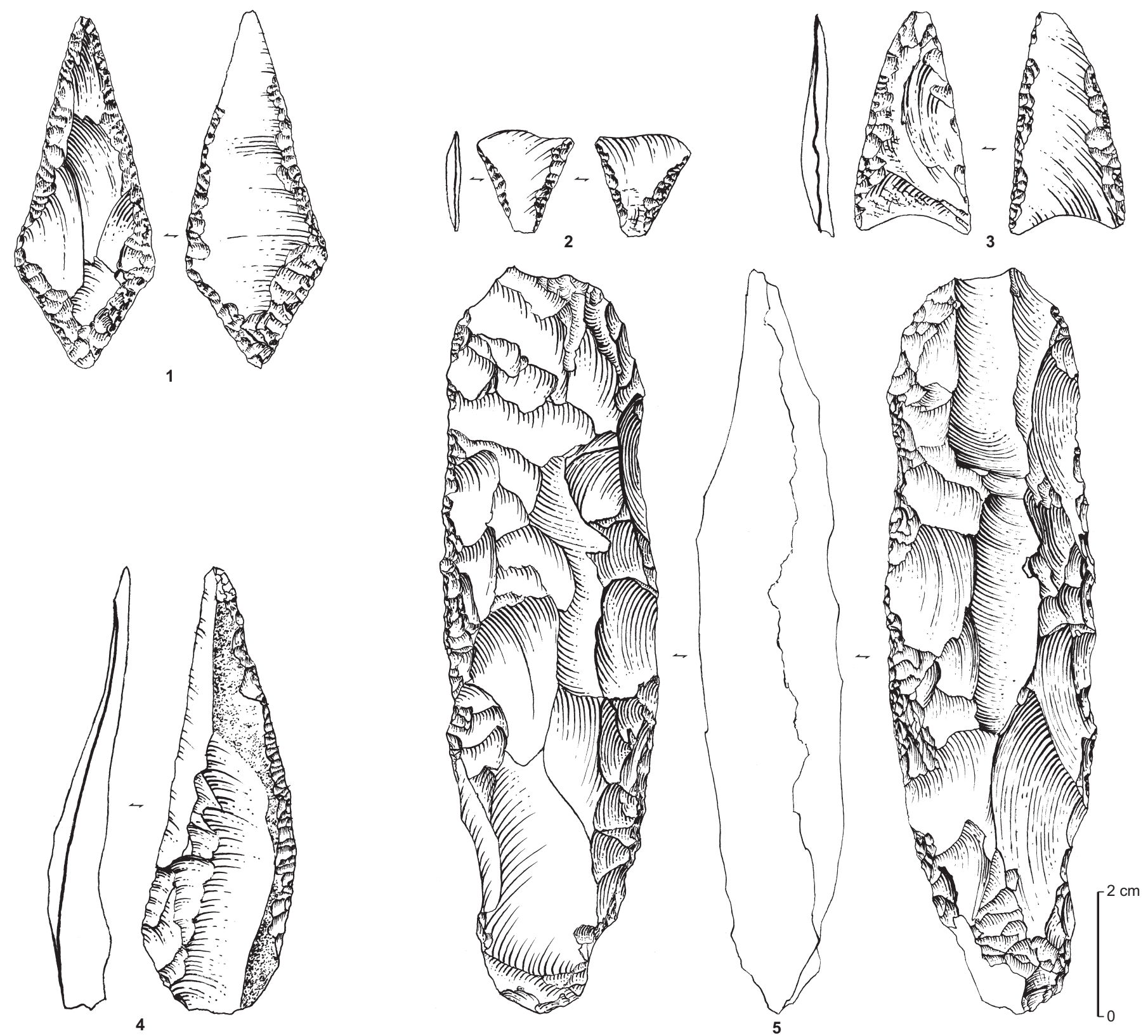

Fig. 11 - Mobilier lithique de la sépulture 2: 1-3, armatures de flèche; 4, racloir; 5, pic-ciseau (dessin: D. Molez, CNRS).

4) Racloir sur éclat laminaire par percussion tendre (talon lisse et mince, corniche abrasée, présence d'une lèvre, bulbe plat): retouche continue sur le bord droit, directe oblique; dimensions: $71 \mathrm{~mm}$ x $23 \mathrm{~mm}$ x $4 \mathrm{~mm}$ (fig. 11, $\mathrm{n}^{\circ} 4$ ).

5) Pic-ciseau: pièce bifaciale à retouche couvrante; une des extrémités est biseautée par l'enlèvement d'un éclat allongé dans l'axe de la pièce; la pièce n'est pas neuve même si elle n'a pas servi à l'issue de sa dernière réfection (voir infra, étude tracéologique, p. 176): d'une part, un écrasement de l'arrête est visible sur l'extrémité opposée au biseau, d'autre part, le négatif d'une cassure en nacelle allongée a provoqué un amincissement trop important de la partie active; dimensions: $120 \mathrm{~mm}$ x $35 \mathrm{~mm}$ x $33 \mathrm{~mm}$ (fig. 11, $\mathrm{n}^{\circ} 5$ ).

La totalité de ces pièces, qui constituent l'essentiel du dépôt funéraire, est en association étroite avec le corps, un adulte masculin. Le racloir était disposé sous l'épaule 


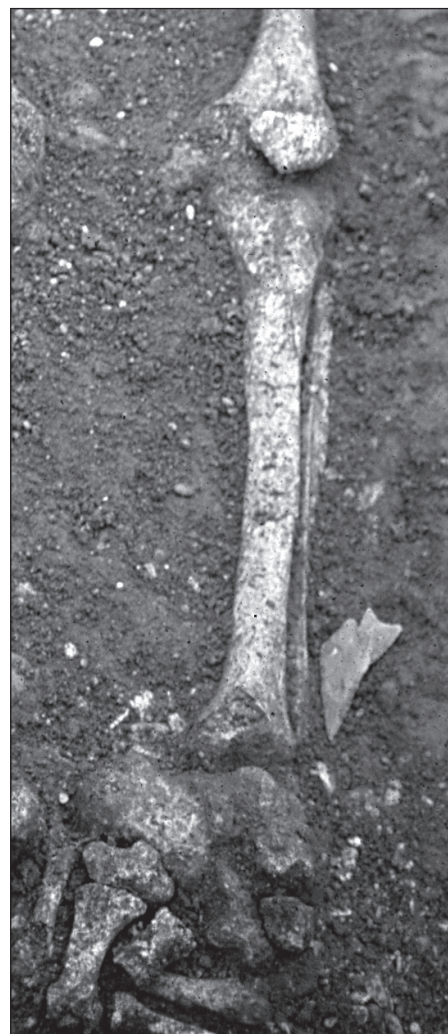

Fig. 12 - Empilement des armatures contre la jambe droite du sujet, dans la sépulture 2. Noter l'orientation de l'armature $n^{\circ} 3$ de la figure 11, confirmant qu'il s'agit bien d'une armature perçante (cliché:J.-L. Tainturier).

droite, extrémité distale vers le sud. Cette position, sur ou sous l'épaule, se retrouve dans les sépultures 3 et 6 , et ne peut être de ce fait considérée comme fortuite: on peut envisager un système de suspension, par exemple une poche ou un sac sur l'épaule.

Les trois armatures étaient regroupées en paquet au niveau de la cheville gauche, partie active vers le sud (fig. 12). On peut penser que les hampes étaient placées le long du mollet. Ces têtes de flèche ne semblent pas avoir bougé depuis leur dépôt; peut-être étaient-elles contraintes dans un contenant, l'hypothèse d'un carquois restant peu envisageable (Chambon, Pétillon, 2009)?

Enfin, le pic-ciseau est localisé sur le poignet gauche de l'individu, perpendiculairement à l'axe du corps, position identique à celle d'un autre pic-ciseau découvert dans une sépulture d'enfant (sépulture 5). On peut se demander, à partir de cette observation effectuée sur deux objets identiques, si ces pièces lithiques ont été enfouies emmanchées dans les tombes, en position fonctionnelle dans la main du mort.

\section{STRUCTURE F2}

La sépulture 2 est distante de $0,70 \mathrm{~m}$ de la sépulture 3 . Entre les deux tombes fut dégagé un amas pierreux enfoui, formant un tas presque circulaire d'une cinquantaine de centimètres de diamètre (fig. 13a). Soixante-six pierres, placées sur cinq niveaux, remplissaient une cavité sur une profondeur de 0,35 m sous le décapage. Au fond, la cavité ne mesure plus que $0,35 \mathrm{~m}$ de diamètre. L'ensemble des pierres, des galets roulés ramassés au bord de la rivière proche (43 en granite, 17 en calcaire jurassique et 6 rognons de silex), pèse environ $50 \mathrm{~kg}$. Les deux rangs supérieurs formaient comme une couronne suggérant le calage d'un poteau qui aurait été appuyé sur les plus grosses placées au fond en rangs serrés. Bien qu'elle soit plus proche de la sépulture 3, le fouilleur rattache cette structure à la sépulture 2: elle se trouve en effet dans le prolongement de son axe central (fig. 13b). Ce dispositif lui suggère la possibilité d'un poteau-marqueur, implanté à la tête d'une sépulture.

\section{SÉPULTURE 3}

La sépulture 3 respecte la même orientation que les sépultures précédentes: nord-ouest/sud-est. La fosse mesure 2,40 m x 1,25 m et paraît largement surdimensionnée pour le corps qui y fut déposé (fig. 14 et 15). Elle mesurait $0,38 \mathrm{~m}$ sous la semelle de labours en son point le plus bas, à savoir au niveau du genou gauche, alors que la tête n'était enfouie qu'à $0,25 \mathrm{~m}$ de profondeur, d'où un pendage marqué. Les bords sont incurvés et le fond de la fosse est arrondi. Le remplissage est constitué de terre brune et un cordon de "petites pierres de 5 à $8 \mathrm{~cm}$ de côté" (Tainturier, 1981), long de 1,10 m s'y trouve noyé parallèlement au corps, le long de son côté droit. La fouille ne mentionne pas un agencement particulier de ces «petites pierres » : l'absence de vue rapprochée ne permet pas d'être aujourd'hui plus précis. Le cordon de pierres, situé à une quinzaine de centimètres du fond, détermine de fait une séparation longitudinale. Dans la partie ouest ainsi réservée, aucun vestige n'a été découvert.

L'extrémité sud de la fosse est légèrement recoupée par une grande fosse moderne (F1). Un éclat laminaire a été mis au jour sur l'avant-bras droit et une lame près de la tête. Deux petites pierres ont été retrouvées (?) de chaque côté de la tête et deux autres au niveau du bassin (Tainturier, 1981) (fig. 16).

Le sujet est orienté comme la fosse, nord-ouest/sud-est, mais est un peu excentré dans la partie orientale de celle-ci. 

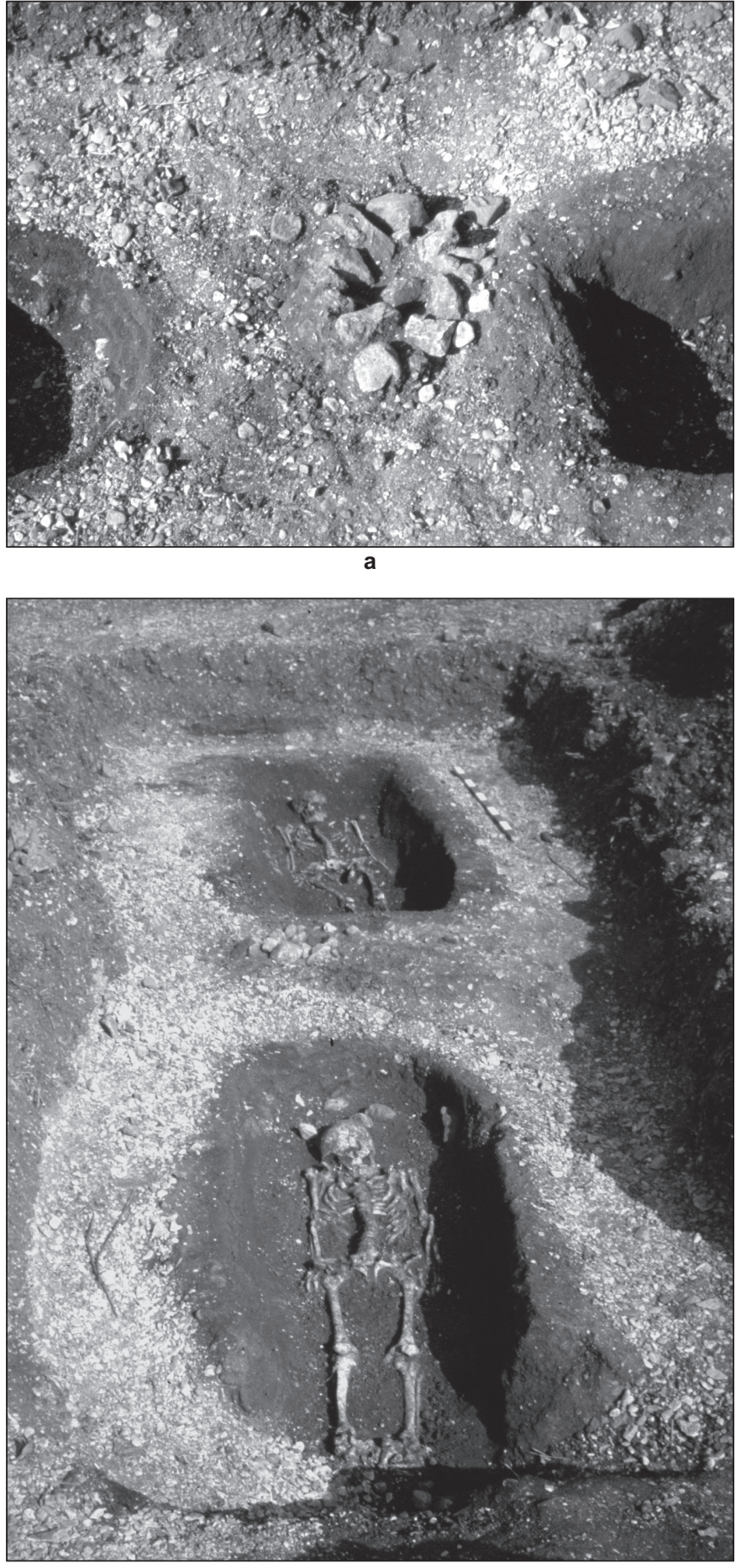

b

Fig. 13 - a, la fosse F2 entre les sépultures $S 2$ (à gauche) et $S 3$ (à droite): en surface du décapage, les galets s'agencent en couronne laissant un petit espace sans pierres au centre de la fosse; J.-L. Tainturier l'interpréta comme un calage de poteau ou de stèle; les bords de la $\$ 2$ sont séparés de la $S 3$ de $70 \mathrm{~cm}$; $\boldsymbol{b}$, vue en perspective des $S 2$, F2 et $S 3$; la F2 se trouve dans le prolongement de l'axe central de la S2 (clichés: J.-L. Tainturier).

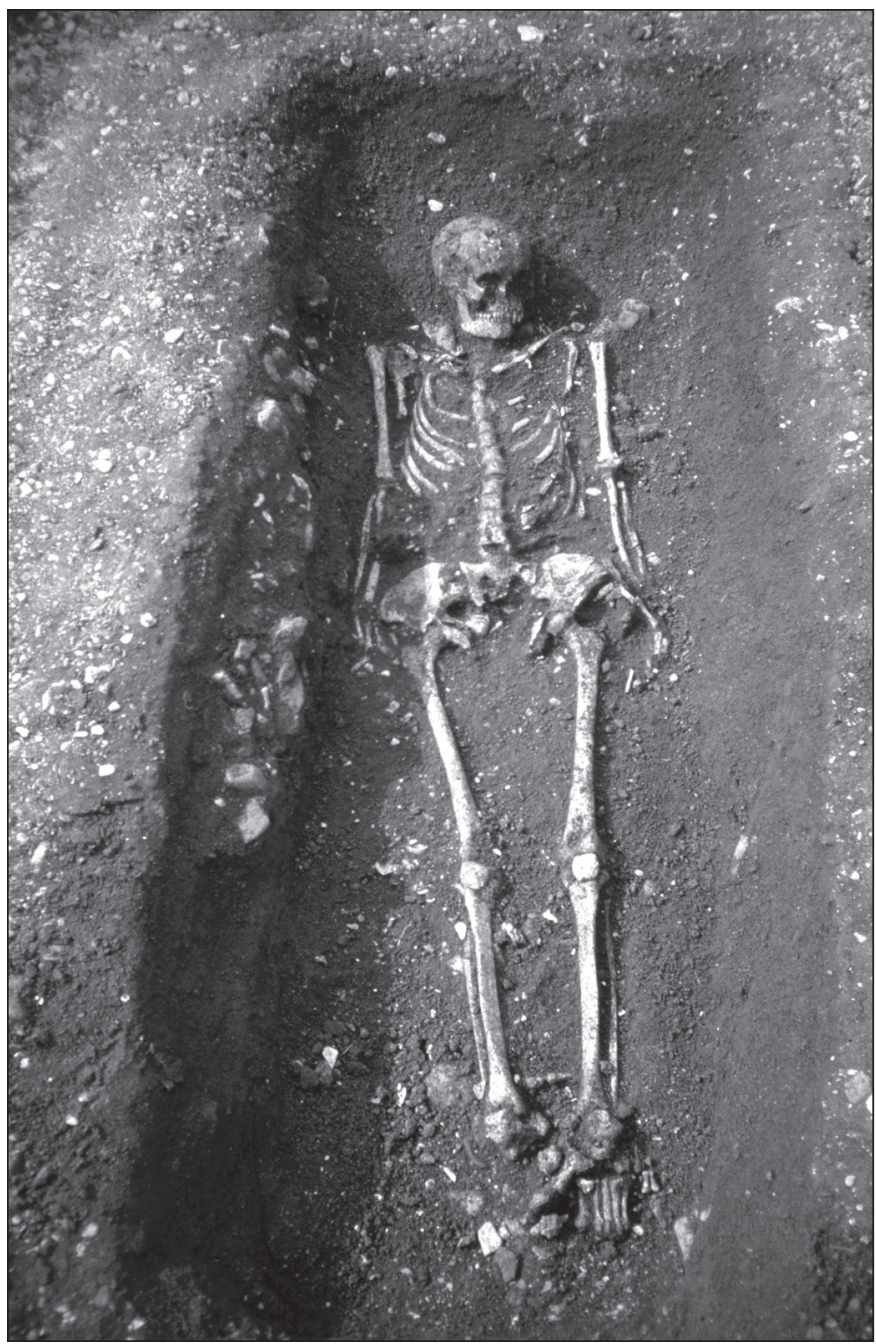

Fig. 14 - La sépulture 3 après dégagement du squelette. Sur le côté droit du sujet, la fouille s'est arrêtée sur le cordon de petites pierres, à plus de 0,50 $\mathrm{m}$ du bord de la fosse (cliché: J.-L. Tainturier).

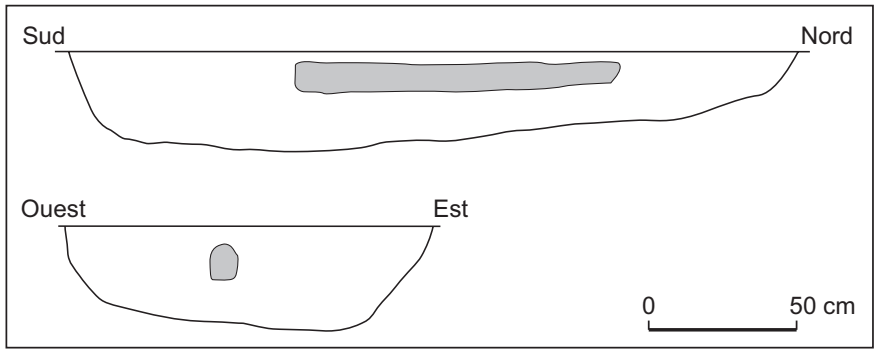

$\square$ accumulation de petites pierres

Fig. 15 - Coupes transversale et longitudinale de la sépulture 3 (DAO: P. Chambon, CNRS). 


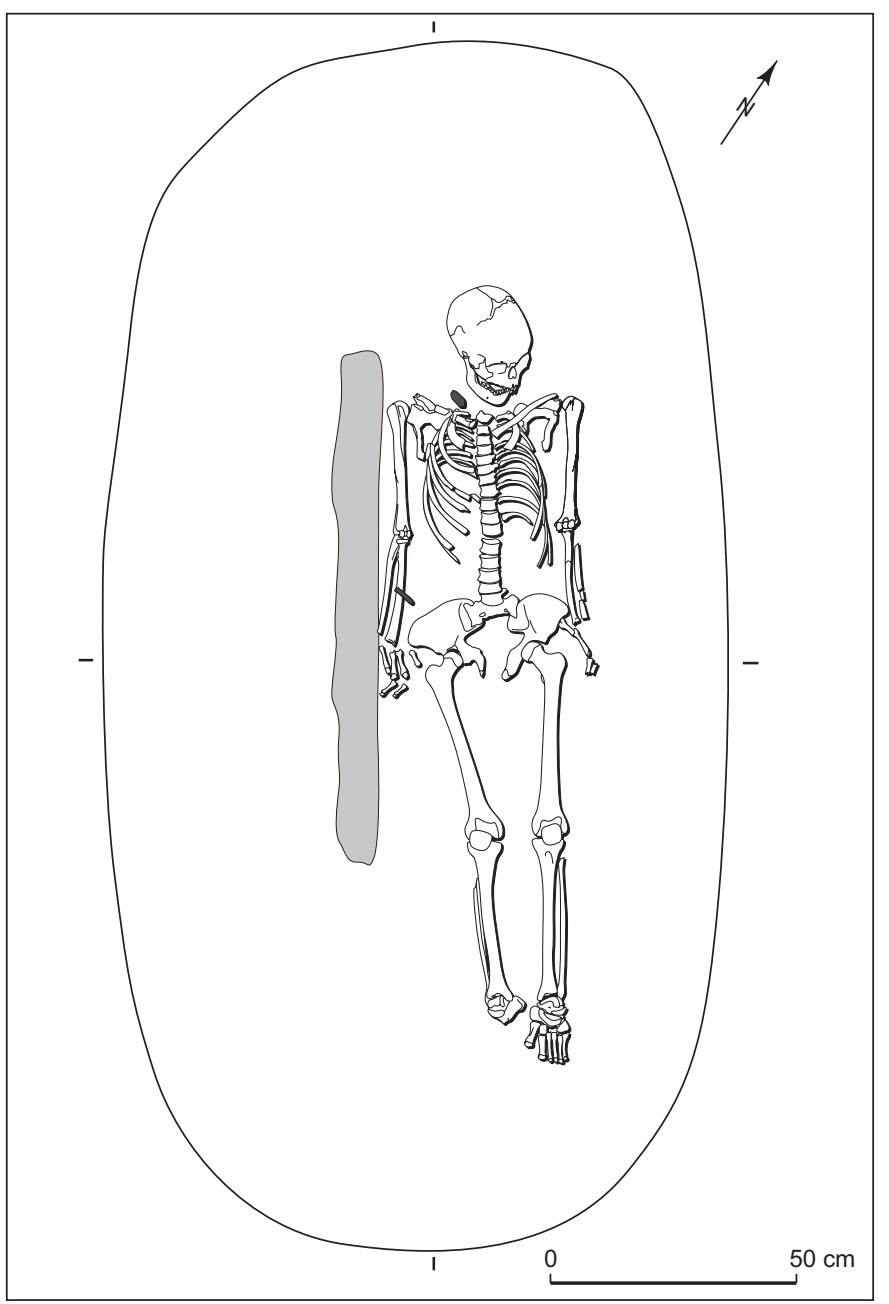

accumulation de petites pierres silex

Fig. 16 - La documentation sur la sépulture 3 est plus lacunaire. Elle n'a fait l'objet que d'un vague croquis dans le rapport de 1981 et les photographies obliques sont plus lointaines que pour la tombe précédente. Le plan que nous présentons a été établi d'après les photographies supplémentaires confiées par $M^{\text {me }}$ Tainturier, que nous avons redressées (relevé: P. Chambon, CNRS).

Il repose en position étendue sur le dos. La tête est légèrement redressée et tournée vers l'épaule gauche. La bouche est fermée. Les membres supérieurs sont étendus le long du corps. Les membres inférieurs sont également étendus, légèrement convergents.

La position du corps ne révèle aucune contrainte, si ce n'est au niveau de la tête: sa position redressée témoigne ou d'un support sous l'occipital ou d'un appui en arrière de celui-ci. Une conception extrémiste des effets de paroi pourrait inciter à décrire un tel effet sur le membre infé- rieur gauche, depuis le pied jusqu'à l'os coxal, non respecté par le membre supérieur.

\section{CONSERVATION ET PERTURBATIONS}

D'après les termes du rapport de 1981, le squelette est «aussi bien conservé» que celui de la sépulture 2. De fait, l'inventaire comprend les os longs et leurs épiphyses, les corps vertébraux, de nombreux os des mains et des pieds... Les grands os plats sont en plus mauvais état, que ce soit le bloc craniofacial, les scapulas ou le sacrum, incomplets.

Les documents graphiques, pas plus que le rapport, ne mettent l'accent sur des perturbations. La position des ossements est cohérente et il n'est nul besoin d'invoquer une tierce intervention pour justifier les écarts à la norme anatomique.

\section{CONNEXIONS ET DISLOCATIONS}

Le bilan taphonomique, succinct, est essentiellement limité aux grandes pièces et finalement peu significatif.

La mandibule est au contact du bloc craniofacial, mais la face s'est affaissée derrière l'arcade dentaire inférieure. L'étage cervical de la colonne n'est pas visible. Celle-ci apparaît à partir de la vertèbre thoracique 3 et se trouve, dès lors, en connexion jusqu'au sacrum. Les côtes visibles sont effondrées dans le volume thoracique.

Les épaules, à plat sur le sol, semblent en connexion. Il en est de même des membres supérieurs dans leur ensemble, sans qu'on ne puisse rien affirmer; les mains paraissent davantage bouleversées.

Le bassin est totalement ouvert. Les hanches semblent, là encore sans plus, en connexion. Les patellas sont en équilibre à cheval sur les fémurs et les tibias. Fémurs et tibias sont en face antérieure. Les chevilles sont disloquées: la trochlée des talus n'est plus au contact de l'extrémité distale des tibias. Les métatarsiens gauches se sont effondrés en bloc, à plat dans l'axe du membre inférieur.

\section{MILIEU DE DÉCOMPOSITION ET TYPE DE STRUCTURE}

Malgré les observations limitées, peut-on évoquer une décomposition en espace vide? Les arguments se réduisent à la mise à plat du bassin, et à la dislocation des pieds. Le parti inverse peut compter sur le basculement incomplet de la tête, et sur l'excellente tenue des membres inférieurs. La situation des patellas, parfaitement symétriques, paraît presque suspecte: telles qu'elles apparaissent sur le cliché, 
elles reposent directement sur les fémurs et les tibias, sans sédiments intercalés: s'agit-il d'un effet de fouille?

La décomposition en espace vide est appuyée par un argument indirect. La bande de petits cailloux, située sur le côté droit du sujet, suggère que la tombe comprend un aménagement, un dispositif architecturé, le sujet a donc bénéficié d'une protection durant la décomposition. La restitution de ce dispositif est plus délicate. La bande de petits cailloux constitue le meilleur indice: si elle est liée à une quelconque paroi, sa position près du centre de la fosse ne milite pas pour un dispositif construit à même la fosse. Enfin, si l'existence de cette bande et sa position permettent indirectement de proposer un contenant mobile, en revanche l'interprétation de ces cailloux reste un problème.

\section{L'INDIVIDU}

Conservation osseuse: la qualité et la représentation osseuses du squelette 3 sont excellentes. Néanmoins, la face de cet individu, consolidée à l'aide de plâtre, limite l'observation de certaines variations anatomiques non métriques à ce niveau.

Âge: la maturation complète de la clavicule et de la crête iliaque nous indique qu'il s'agit d'un individu adulte d'au moins 25 ans. L’observation des quatre critères dégénératifs de la surface sacro-pelvienne situe la limite supérieure de cet âge à 50 ans.

Sexe: le rapport de 1981 décrit le sujet 3 comme une femme, ce que nous confirmons au vu de la morphologie des os coxaux d'après les trois premiers critères de la méthode morphoscopique (région préauriculaire, grande incisure ischiatique et arc composé) et du résultat de la DSP. Le «caractère féminin" du bloc craniofacial est ainsi cohérent avec le diagnostic établi à partir des os coxaux.

Stature: la mesure des fémurs de cet individu nous a permis d'estimer sa stature à 1,50 m environ, légèrement inférieure à l'estimation initiale de 1,55 m (Tainturier, 1981).

État buccodentaire: à l'exception des deux incisives inférieures droites, toutes les dents sont observables. L'usure globale est limitée, voire absente pour les quatre troisièmes molaires, ainsi que pour les deuxièmes molaires et prémolaires supérieures gauches. Une importante lésion carieuse atteint la face distale de la première molaire inférieure gauche, s'étendant du tiers moyen de la couronne au sommet de la racine. Les racines sont légèrement exposées sur les premières et deuxièmes molaires inférieures, indiquant une légère parodontose à ce niveau. Le dépôt de tartre, absent sur l'ensemble des dents antérieures, est léger sur les dents postérieures et systématiquement installé au niveau du collet.

Indicateurs de stress biologique: seule la première incisive supérieure gauche présente nettement deux hypoplasies linéaires de l'émail dentaire, réparties sur les tiers moyen et cervical de la couronne.

Variations anatomiques: en dehors de variations anatomiques non métriques cotées de façon systématique pour chaque squelette, l'individu 3 présente l'inclusion totale de la canine inférieure droite.

\section{UN INDIVIDU SURNUMÉRAIRE : 3BIS}

En faisant l'inventaire des ossements de ce sujet, nous avons eu la surprise de retrouver cinq restes osseux ne lui appartenant manifestement pas. Les plus caractéristiques se trouvaient associés à la main gauche: il s'agit de deux fragments de fémur gauche (extrémité distale) de très petite dimension. Avec la main droite, ce sont trois esquilles que nous n'avons pu identifier avec certitude (fibula et tibia?), mais qui potentiellement peuvent être issues du même squelette. L'estimation de la longueur diaphysaire est impossible, empêchant l'attribution directe d'un âge précis. La comparaison de ces fragments aux autres individus immatures de la nécropole démontre un format inférieur à celui de l'individu 11 (le plus jeune sujet de l'ensemble funéraire, classé parmi les $\left[\begin{array}{ll}0 & \mathrm{an}\end{array}\right]$ ). Nous avons comparé ces fragments à de très jeunes sujets issus d'autres collections ostéologiques. Il semble vraisemblable que cet individu soit décédé en période périnatale, potentiellement avant la naissance.

Les documents en notre possession ne font aucunement mention d'un sujet associé à l'adulte. Il est curieux que ces restes soient si peu nombreux. Le conditionnement minutieux des ossements que nous avons recueillis auprès de l'épouse du fouilleur ne milite pas pour un mélange fortuit, mais il n'est plus possible d'en être sûr. Si ces restes proviennent bien de la sépulture 3, le fait qu'ils n'aient pas été remarqués sur le terrain suggère qu'ils étaient étroitement associés à l'adulte. L'identité biologique de ces deux sujets (une femme adulte et un enfant probablement mort avant la naissance) soutient fortement cette hypothèse.

\section{LE MOBILIER}

1) Lame à deux pans, sens de débitage unidirectionnel ; le talon est lisse et dièdre; une de ses facettes pourrait correspondre à un négatif d'enlèvement opposé, auquel cas il pourrait s'agir, sans certitude toutefois, d'un éclat de pré- 


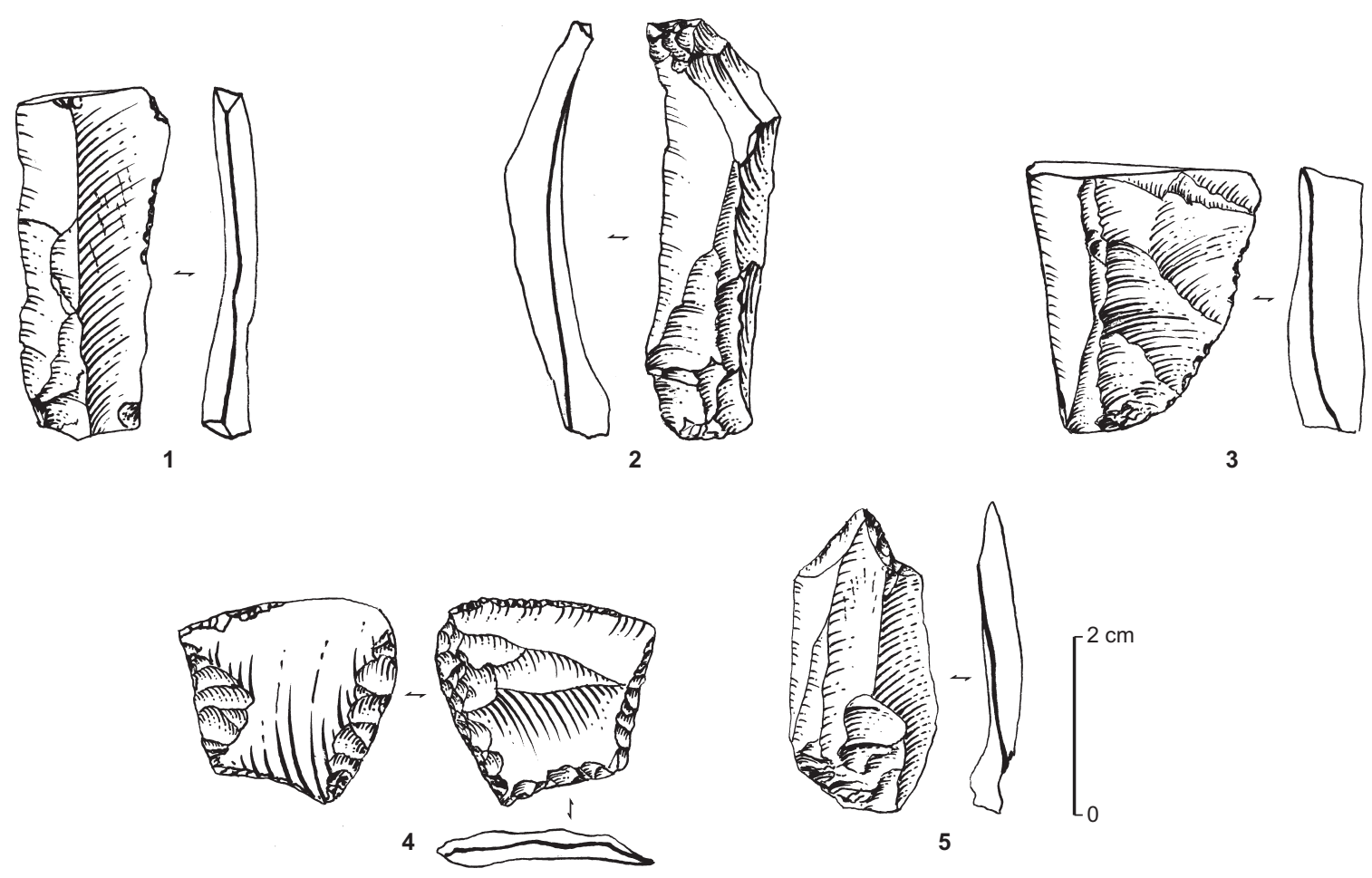

Fig. 17 - Mobilier lithique: 1, lame de la sépulture 3; 2, éclat laminaire de la sépulture 3; 3, fragment d'éclat de la sépulture 8; 4, armature de la sépulture 10; 5, coche sur lame de la sépulture 12 (dessin: D. Molez, CNRS).

paration de crête ; l'extrémité distale manque; dimensions : $38 \mathrm{~mm}$ x $17 \mathrm{~mm}$ x $5 \mathrm{~mm}$ (fig. 17, $\mathrm{n}^{\circ} 1$ ).

2) Éclat laminaire, dont la modalité de détachement ne peut être formellement identifiée; le talon est linéaire, la corniche est abrasée; l'extrémité distale manque; dimensions: $47 \mathrm{~mm}$ x $13 \mathrm{~mm}$ x $8 \mathrm{~mm}$ (fig. 17, $\mathrm{n}^{\circ}$ 2).

Ces deux pièces forment l'unique dépôt d'objets associé à l'inhumé. La première est située à proximité de l'épaule droite, comme le racloir et les éclats des sépultures 2 et 6 . La seconde est en position perpendiculaire par rapport à l'axe du corps, sur l'avant-bras droit.

\section{SÉPULTURE 4}

Dans la continuité des sépultures 2 et 3, la sépulture 4 suit le même alignement nord-ouest/sud-est. Son extrémité sud fut appréhendée lors de la fouille de 1981 mais la tombe ne fut fouillée qu'en 1983.

La fosse présente une forme irrégulière, allongée, ni vraiment rectangulaire, ni elliptique (fig. 18). Elle a pour dimensions $2,25 \mathrm{~m}$ x $0,95 \mathrm{~m}$ et le creusement est plus profond au nord et à la tête $(0,70 \mathrm{~m}$ sous le sol actuel) qu'au niveau des pieds $(0,50 \mathrm{~m})$. Ainsi le bord de la fosse est presque vertical au nord alors qu'il descend en pente douce à l'opposé. Le remplissage est constitué d'une terre brune, fine, exempte de graviers. Toutefois, onze pierres d'un petit module sont disposées immédiatement auprès du corps et sur celui-ci. Comme leur nombre est restreint et qu'on ne les trouve qu'au contact du cadavre, on peut penser à une démarche intentionnelle. De même, un dépôt organique grisâtre a été observé sur la partie gauche du corps, le long du bord nord de la fosse et sur la poitrine. Mêlé de terre, il est plus abondant au niveau du coude et du genou.

La sépulture 4 correspond à l'inhumation individuelle d'un sujet adulte, allongé sur le dos, la tête au nord-ouest et les pieds au sud-est. L'éclatement du bloc craniofacial ne permet pas d'être affirmatif quant à la position initiale de la tête: en vue antérieure? légèrement supérieure? ou en face latérale droite? Les membres sont étendus dans l'axe du corps, à l'exception de l'avant-bras gauche replié sur le ventre.

L'espace occupé par le squelette a une limite irrégulière du côté droit. En revanche, du côté gauche, aucun ossement n'outrepasse la ligne formée par l'os frontal et les diaphyses de l'humérus et du fémur. 

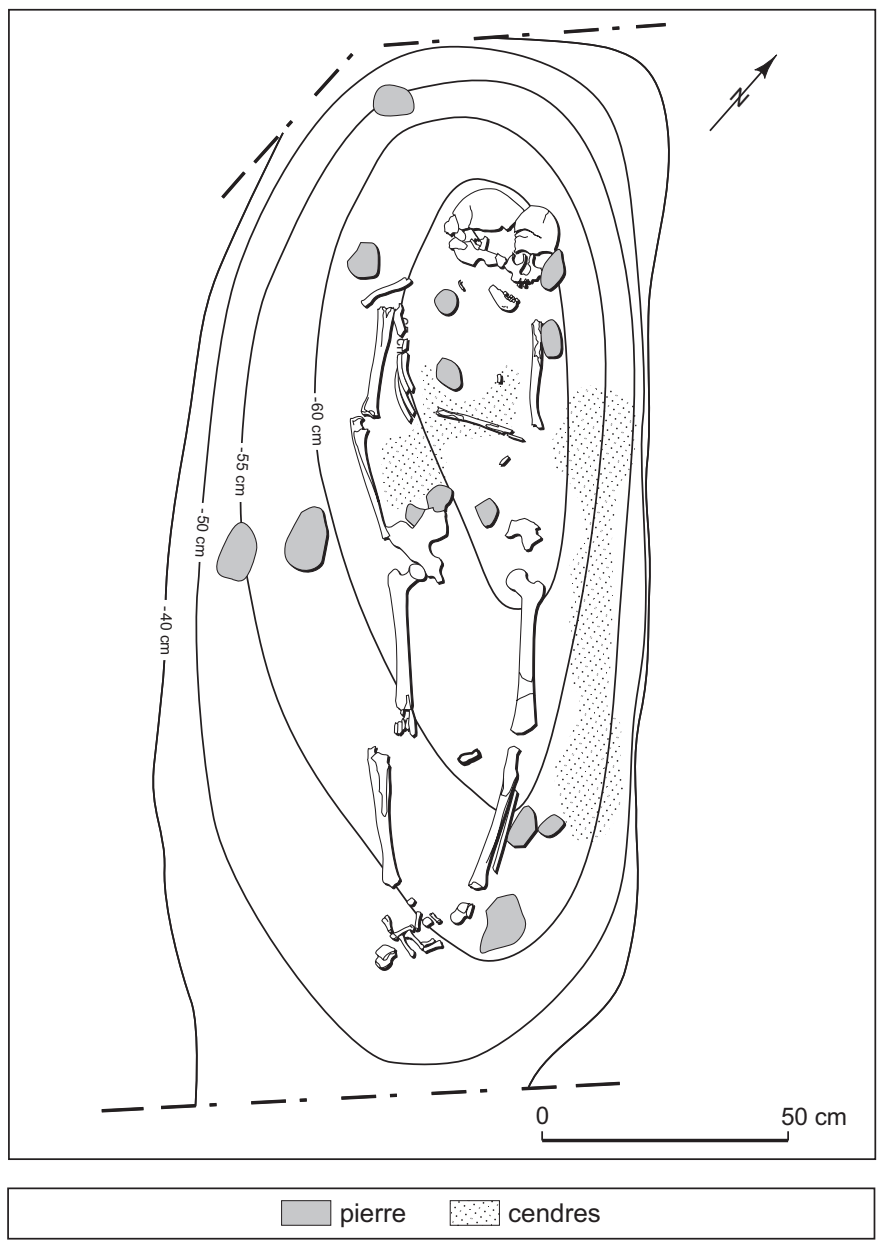

Fig. 18 - Plan de la sépulture 4. L'altitude 0 correspond au sol actuel (relevé: P. Chambon, CNRS).

Deux lames de silex cassées étaient intégrées au remplissage, sur la poitrine et l'abdomen. Une troisième fut retrouvée près du mollet gauche.

\section{CONSERVATION ET PERTURBATIONS}

L'état général des ossements est mauvais, voire très mauvais (fig. 19). La fouille n'a mis au jour qu'une quarantaine de restes dont peu sont complets. Le bloc craniofacial est la partie du squelette la mieux préservée, bien que la surface de l'os soit érodée; la mandibule se restreint à trois fragments disjoints. Les principaux os longs sont privés de leurs épiphyses: seules subsistent l'extrémité proximale de l'ulna droite, l'extrémité proximale du fémur droit (ainsi qu'une trace de son symétrique) et l'épiphyse distale du tibia droit. Parmi les autres vestiges figurant à l'inventaire,

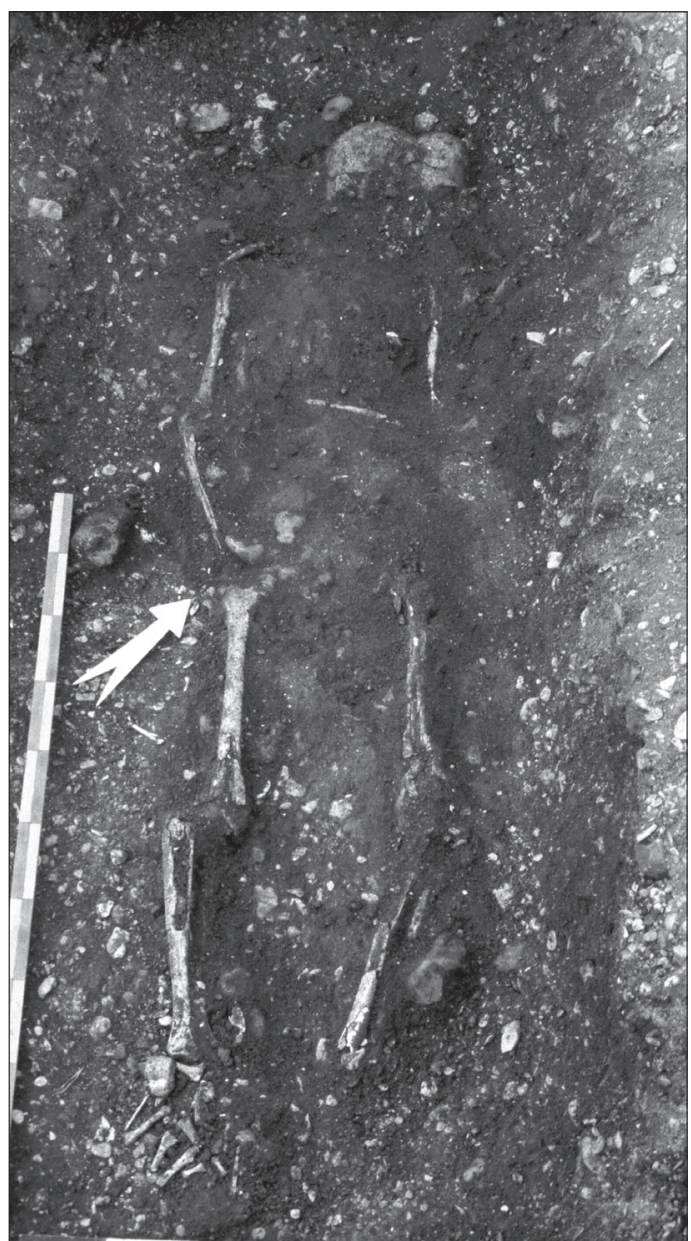

Fig. 19 - Vue du squelette de la sépulture 4. Noter l'éclatement du crâne. Malgré le très mauvais état général des ossements, les dislocations apparaissent nettement (cliché: J.-P. Delor).

il faut souligner la mauvaise représentation des os des ceintures, hormis la clavicule droite. Quelques esquilles de côtes droites sont les uniques témoins du thorax.

La conservation des vestiges mis au jour est telle qu'il n'est nul besoin d'invoquer des interventions extérieures pour expliquer les manques. Malgré les libertés prises par les ossements avec l'anatomie, on ne distingue aucune aberration impliquant la patte de l'animal ou la main de l'homme. Le tableau offert est bien celui laissé par la décomposition du cadavre au sein de la sépulture.

\section{CONNEXIONS ET DISLOCATIONS}

Le bilan des articulations est forcément réduit: nous l'avons établi d'après les photographies et il ne peut concerner qu'une faible partie du squelette. La détermination du 
milieu de décomposition ne pose toutefois aucune difficulté.

Le volume cérébral n'est pas préservé. Le bloc craniofacial est aplati et, quelle que soit sa situation à l'origine, cet affaissement outrepasse son volume initial. La mandibule, dont seul un fragment est visible sur les photographies (en face antérosupérolatérale droite), ne peut plus être en connexion avec l'os maxillaire et les os temporaux.

La clavicule droite est la pièce la plus éloignée de sa situation anatomique: elle s'est déplacée latéralement de plus de $7 \mathrm{~cm}$. Le coude droit est disloqué: l'humérus comme l'ulna reposent en vue antérolatérale, mais l'olécrane est nettement décroché de l'humérus. Du côté gauche, on note seulement la situation déséquilibrée de l'humérus qui repose en face latérale.

Au sein des membres inférieurs, la situation des cuisses contraste avec celle des jambes. Les fémurs sont situés dans l'axe du corps, parallèles entre eux, et en vue antérieure. La jambe droite a pivoté latéralement, rompant l'articulation du genou; le pied est disloqué, mais la position des ossements présents indique qu'il a suivi la jambe dans sa rotation. La jambe gauche a pivoté et apparaît en face médiale, toujours dans le prolongement du fémur.

\section{MILIEU DE DÉCOMPOSITION ET TYPE DE STRUCTURE}

Malgré le petit nombre d'articulations analysables, les observations convergent pour conclure à une décomposition du cadavre dans un milieu non colmaté. Tant au niveau du bloc craniofacial, que de l'épaule droite, du coude droit, de la jambe et du pied droits, des ossements ont largement outrepassé le volume initial du corps. De tels mouvements impliquent un vide durant la décomposition.

Si la condition du vide est indispensable, elle ne justifie pas des mouvements d'une telle ampleur. L'éclatement du bloc craniofacial suggère une rupture brutale de la couverture. Cet événement a pu également mobiliser quelques vestiges sur le fond de la sépulture. On ne saurait utiliser un tel argument pour expliquer le déplacement latéral des ossements sur la bordure droite du sujet, glissement général et non phénomène ponctuel. Ces ossements n'ont pas glissé sur le fond de la fosse: la partie la plus profonde est au centre, sous le tronc. De fait, il nous faut introduire, entre le cadavre et le fond de la fosse, un élément rigide dont la surface est à peu près lisse et qui était affecté d'une pente suffisante durant la décomposition pour justifier le glissement des ossements.
L'existence d'un élément en matière périssable sous le corps trouve un écho avec l'effet de paroi détecté sur le côté gauche du squelette: cet effet est souligné par la position instable de l'humérus (on peut discuter du rôle de la pierre placée contre lui). Ces deux témoignages, fond et montant, suggèrent que le corps ait reposé dans un contenant rigide et amovible. Les pierres, peu nombreuses, n'avaient sans doute pas de réelle fonction dans la structure, toutefois celles situées dans l'entourage du squelette appuyaient sur les parois du contenant. L'évolution du corps dans un espace vide implique une structure fermée, soit le contenant amovible possédait un couvercle, soit c'était la tombe elle-même. Les traces cendreuses (en fait, organiques) entre le côté gauche du sujet et la paroi de la fosse constituent l'unique (et faible) indice en faveur d'une couverture sur la tombe, ou d'une architecture autre que le contenant lui-même.

\section{L'INDIVIDU}

Conservation osseuse: le sujet 4 est très mal représenté. Les fragments de la tête osseuse n'ont permis qu'un remontage très partiel. Les fragments de l'os maxillaire et de la mandibule permettent l'observation de plusieurs dents in situ. Le squelette intracrânien n'est représenté que par l'extrémité distale des humérus, le tiers proximal de l'ulna droite, la moitié distale du tibia droit et quelques os fragmentés du pied droit. Les os coxaux ne sont représentés que par une partie des tubérosités ischiatiques et un fragment de l'aile iliaque au niveau du bord supérieur.

Âge: le fragment de l'aile iliaque présente une crête complètement soudée suggérant la présence d'un individu adulte d'au moins 20 ans. Cette observation est cohérente avec le reste des fragments osseux comportant des points d'ossification secondaire (humérus, tibia, calcanéus). En outre, la synostose de la suture lambdoïde est entamée.

Sexe: l'état des os coxaux ne nous permet aucune diagnose sexuelle. En aucun cas, les trois «critères féminins" observés sur le bloc craniofacial ne nous permettent de conclure quant au sexe de l'individu.

État buccodentaire: vingt et une dents (dont sept isolées) sont conservées. À l'exception de la première prémolaire supérieure droite dont la couronne fait complètement défaut, l'ensemble des dents observables présente une usure limitée, voire absente pour les quatre deuxièmes et troisièmes molaires. Aucune carie n'est clairement identifiable, bien que l'usure inhabituelle de la P1 supérieure droite nous laisse envisager qu'une importante lésion 
carieuse en soit à l'origine. Les dents in situ ne témoignent d'aucune maladie parodontale; quant au dépôt de tartre, il n'a été observé de façon légère que sur trois dents postérieures.

Indicateurs de stress biologique: nous identifions nettement douze hypoplasies linéaires de l'émail dentaire réparties sur seulement huit dents. Les cinq dents antérieures supérieures conservées sont les plus atteintes en terme de sévérité et de quantité d'hypoplasies observables par dent. Ainsi les incisives et la canine droite présentent une atteinte à la fois sur les tiers moyen et cervical de la couronne. Les deuxièmes molaires observables ne sont atteintes qu'au niveau du tiers moyen de la couronne.

Variations anatomiques: en dehors des variations anatomiques non métriques cotées systématiquement pour chaque individu, le sujet 4 présente une inclusion totale de sa canine inférieure gauche.

\section{LE MOBILIER}

1) Fragment proximomésial de lame par percussion tendre (talon lisse, mince et incliné, corniche abrasée, présence d'une lèvre, bulbe plat); lame à plus de trois pans, sens de débitage unidirectionnel; dimensions: $48 \mathrm{~mm}$ x $19 \mathrm{~mm}$ x $4 \mathrm{~mm}$.

2) Éclat partiellement cortical dont manque l'extrémité distale, débité par percussion dure (cône incipient visible).

3) Fragment distal d'éclat portant quelques retouches denticulées.

L'association des pièces lithiques avec l'inhumation ne paraît assurée que pour le denticulé, retrouvé à proximité du mollet gauche.

\section{SÉPULTURE 5}

La sépulture 5 fait partie du groupe des sépultures dégagées à l'est du décapage. Son creusement est parfaitement délimité dans le gravier et présente une forme elliptique: son grand axe mesure 2,20 m pour une largeur de 1,35 m. Elle est exactement orientée nord-sud et jointive au sud avec la sépulture 4.

Le remplissage de terre fine et très brune, sans gravier, est semblable à celui des sépultures précédentes. À son sommet, juste sous le décapage, fut observé, placé au point central de la tombe, le petit dépôt d'une lame, d'un éclat de silex chauffé (voire calciné) et d'un fragment de cristal de roche portant des traces de façonnage. Ce dernier matériau, très rare en nos contrées, est connu dans une autre sépulture, celle des Plantes du Mont à Gurgy (Delor, 1991). Quelques galets de granite sont alignés sur le bord du remplissage à l'est.

$\mathrm{Au}$ fond de la fosse, à - 0,57 m, un surcreusement aux dimensions plus modestes se dessine $(1,60 \mathrm{~m}$ x $0,90 \mathrm{~m})$. Là encore, cinq galets se trouvent en bordure du nouveau remplissage qui, pourtant, se présente de façon homogène du haut en bas de la tombe. Le surcreusement atteint la profondeur de $0,74 \mathrm{~m}$ et le fond montre une légère surélévation dans sa moitié sud (fig. 20). Une quinzaine de galets en chaille ou en granite étaient disséminés sur le fond mais forment un alignement évident à l'ouest, le long du squelette (fig. 21). En revanche, à l'est, a été observé un dépôt grisâtre, probablement d'origine organique, placé avant rebouchage.

La présence de deux creusements «emboîtés» ne permet pas d'affirmer la vocation initiale de la fosse. La seule

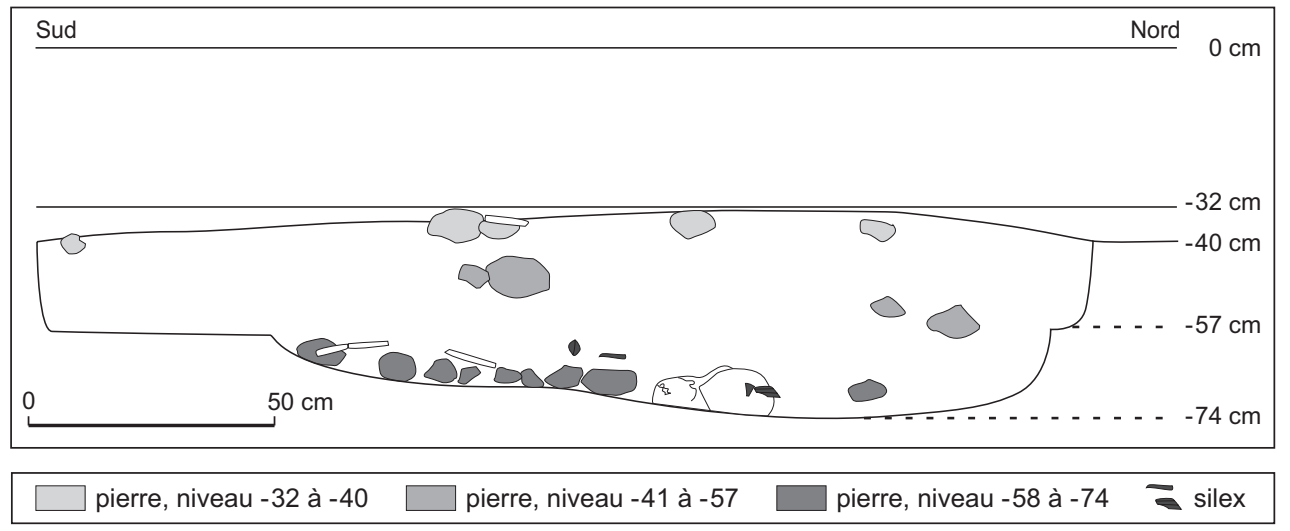

Gallia Préhistoire, 52, 2010, p. 117-192
Fig. 20 - Coupe longitudinale de la sépulture 5. Le niveau - 57 marque une rupture dans le profil, correspondant à un surcreusement plus étroit de la fosse initiale (relevé: P. Chambon, CNRS et J.-P. Delor). 
destination qu'on puisse identifier est l'inhumation d'un sujet immature en position allongée sur le dos, dans le grand axe de la fosse, la tête au nord et les pieds au sud. La tête est redressée, la bouche est fermée. Les restes osseux comme le mobilier mis au jour n'occupent qu'une petite surface au sein de la fosse: on peut la ramener à un rectangle de 0,80 $\mathrm{m}$ de long pour 0,20 $\mathrm{m}$ de large.

L'inventaire du mobilier issu de la sépulture 5 contient neuf pièces lithiques. Parmi elles, sept ont été mises au jour au niveau de l'inhumé: quatre armatures de flèche à tranchant transversal entre la jambe gauche et l'alignement des pierres, une lame de silex se trouvait au niveau de la poitrine et un pic-ciseau à celui de l'avant-bras gauche, tranchant à l'ouest (fig. 22).

\section{CONSERVATION ET PERTURBATIONS}

La très mauvaise conservation des ossements réduit l'inventaire à une poignée de vestiges. Parmi eux, seul le bloc craniofacial et la mandibule sont présents en quasi-intégralité. Les autres ne sont que des esquilles, généralement non identifiables.

L'état des ossements ne permet ni de supposer ni d'exclure d'éventuelles perturbations. Les vestiges osseux mis au jour sont concentrés dans la zone où reposait le corps. La disposition du mobilier lithique suggère toutefois deux remarques. Un éclat laminaire est brisé en deux morceaux écartés de $6 \mathrm{~cm}$; il se situe vers le haut de l'abdomen. Une pile d'armatures de flèche a été mise au jour près de la tête, du côté droit du sujet, trois sont orientés parallèlement, indiquant que les hampes se trouvaient parallèlement au corps; l'orientation de la dernière ne correspond pas et traduit un déplacement après la rupture de la hampe.

\section{CONNEXIONS ET DISLOCATIONS}

L'analyse des liens articulaires n'est possible qu'au niveau de la tête. Le bloc craniofacial est disloqué. Les os qu'on distingue sur les photographies (os pariétaux, os frontal, os maxillaires) ont repris leur autonomie. Le volume cérébral s'est résorbé, mais l'affaissement de la voûte s'est accompagné de l'étalement des ossements. Les os maxillaires et la mandibule sont restés solidaires.

\section{MILIEU DE DÉCOMPOSITION ET TYPE DE STRUCTURE}

L'étalement partiel des os crâniens indique un vide dans cette région durant la décomposition. Les restes osseux ne

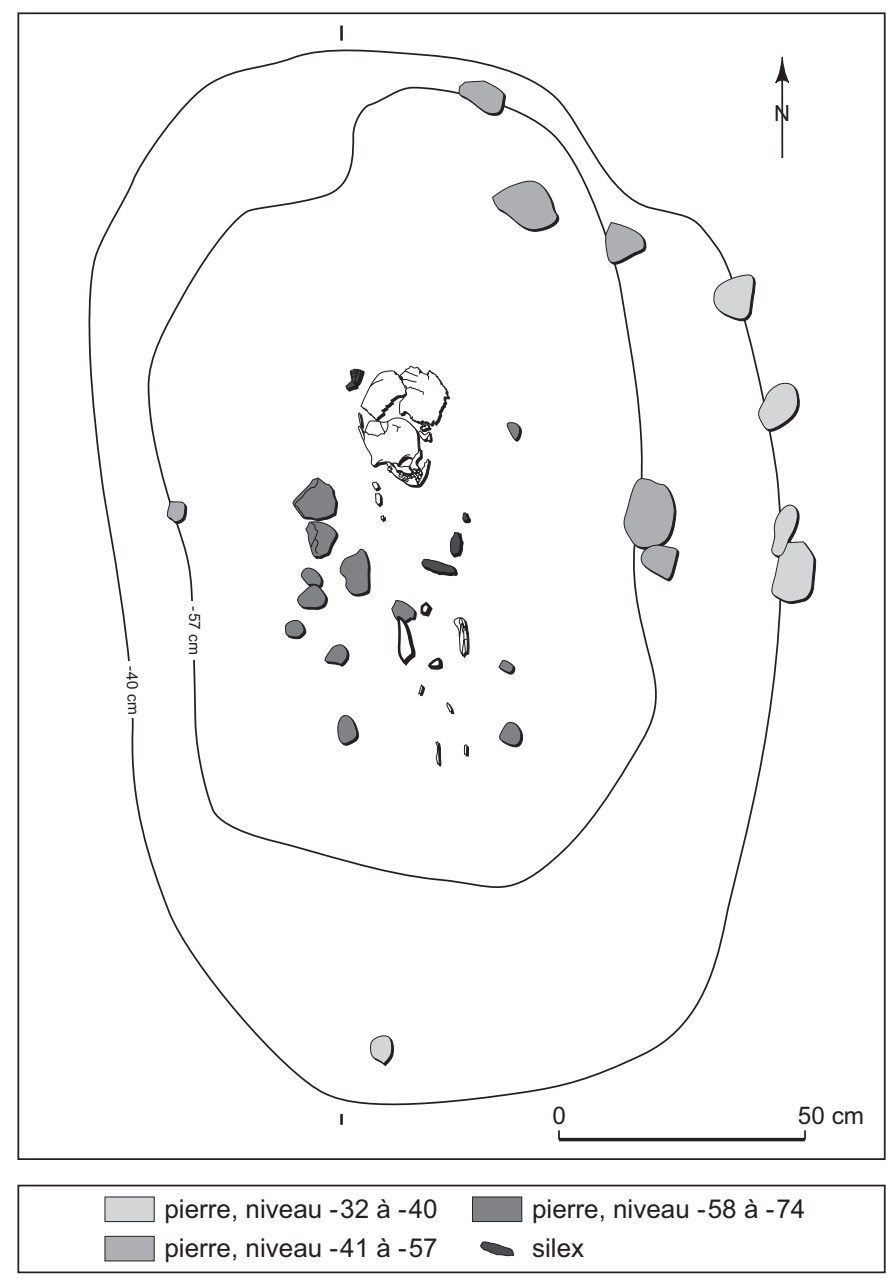

Fig. 21 - Plan de la sépulture 5 (relevé: P. Chambon, CNRS).

permettent pas d'en dire plus. Le mobilier, les pierres et la fosse elle-même autorisent quelques commentaires. Le plan montre une nette disjonction entre les vestiges osseux et le mobilier lithique, d'une part, et les galets, d'autre part (un seul se trouve au milieu des ossements). Nous l'avons signalé, ossements et mobiliers sont circonscrits dans un espace rectangulaire étroit. Enfin, la fosse est beaucoup plus importante.

L'hypothèse que cet espace rectangulaire corresponde à un contenant (rigide mobile) n'est guère osée. Une telle disproportion entre ce contenant et le creusement n'interdit pas l'existence d'autres aménagements à même la fosse : les pierres en sont peut-être l'ultime témoignage.

Le mobilier lithique est étroitement associé au sujet selon trois modalités. Le petit outil sur bloc (pic ou ciseau) comme l'éclat laminaire reposaient directement sur le sujet, ou étaient, plus simplement, portés: ils se trouvaient 


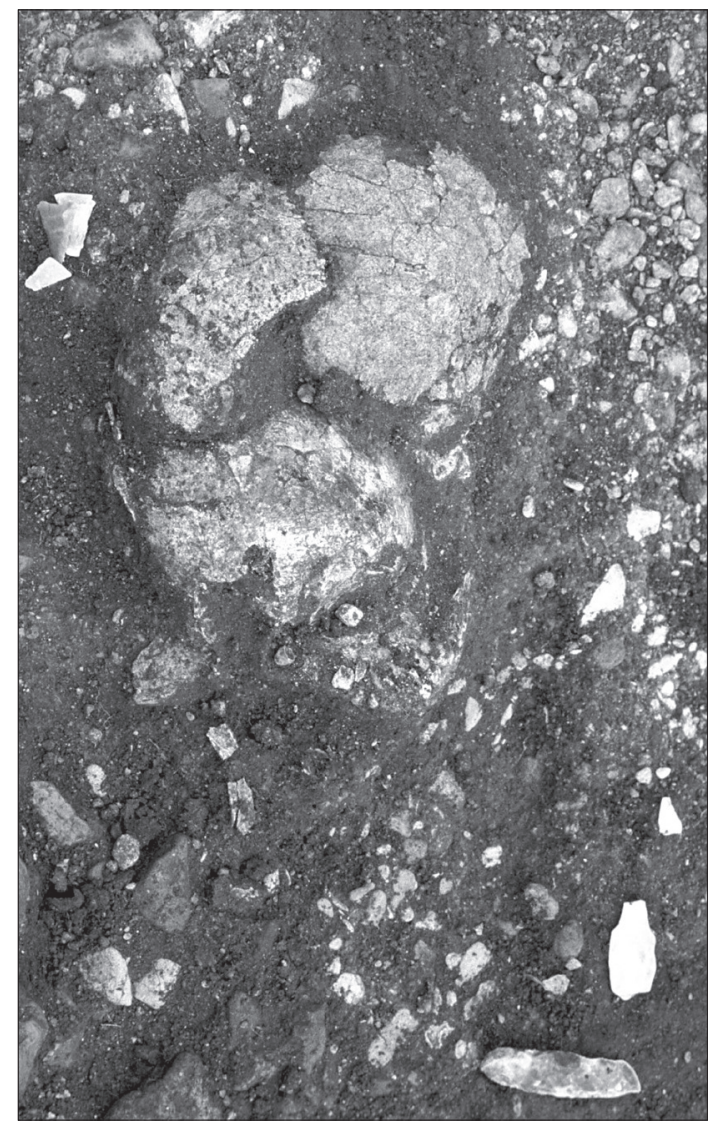

Fig. 22 - Vue de la partie supérieure du squelette. Noter l'empilement des armatures de flèche à la droite du crâne et le pic-ciseau positionné comme dans la sépulture 2 (cliché:J.-P. Delor).

donc au sein du contenant. Les flèches sont disposées le long du corps: telles qu'on peut les restituer, les hampes divergent progressivement du sujet; on ne peut cependant pas rejeter la possibilité d'un léger basculement de la pile de flèches (l'une d'elles est perpendiculaire). Au final, il subsiste une alternative: soit les flèches étaient incluses dans le contenant, soit elles ont été déposées directement dans la tombe, contre la paroi du contenant. Le dernier lot de pièces lithiques a été mis au jour plus de $0,30 \mathrm{~m}$ au-dessus du squelette: un éclat laminaire, un éclat de silex calciné et un fragment de cristal de roche. Cet ensemble se trouve néanmoins, en plan, dans l'aire du contenant et, à moins d'invoquer le hasard, devait reposer sur la couverture.

\section{L'INDIVIDU}

Conservation osseuse: les restes osseux de cet individu n’ont pas été retrouvés.
Âge: les relevés de terrain nous ont permis d'estimer la longueur maximale de la fosse à 1,50 m. L'extrémité sommitale du bloc craniofacial repose à environ $0,50 \mathrm{~m} \mathrm{du}$ bord de la fosse. La stature de l'individu ne semble donc pas pouvoir dépasser le mètre. La taille de cet individu correspond, selon les tables de M. Sempé et G. Pedron (1979), à un enfant (garçon ou fille) âgé entre 3,5 et 5,5 ans. Selon notre objectif d'une répartition des individus immatures en classe d'âge quinquennale, nous pensons donc raisonnable de classer cet individu dans l'une ou l'autre des deux classes, [1-4] ou [5-9] ans.

\section{LE MOBILIER}

1) Armature de flèche tranchante trapézoïdale, peutêtre sur support laminaire (les enlèvements lisibles sur la face supérieure sont parallèles et de même direction que le sens de débitage du support), en silex à grain très fin; la retouche, bifaciale, s'est effectuée en deux temps principaux : retouche marginale sur face inférieure, puis retouche oblique de la face supérieure; dimensions : $18 \mathrm{~mm}$ x $17 \mathrm{~mm}$ x 2,5 mm (fig. 23, $\mathrm{n}^{\circ} 1$ ).

2) Armature de flèche tranchante triangulaire sur éclat mince; l'organisation de la retouche est identique à celle des $n^{\text {os }} 3$ et 4 : d'abord la face inférieure, puis la face supérieure; dimensions: $28 \mathrm{~mm}$ x $21 \mathrm{~mm}$ x $3 \mathrm{~mm}$ (fig. 23, $\mathrm{n}^{\circ} 2$ ).

3) Armature de flèche tranchante trapézoïdale sur éclat mince; la retouche, bifaciale, a été réalisée de façon alterne: retouche directe par le bord droit et inverse par le bord gauche, puis inverse par le bord droit et directe par le bord gauche; dimensions: $22 \mathrm{~mm}$ x $20 \mathrm{~mm}$ x $3 \mathrm{~mm}$ (fig. 23, $\mathrm{n}^{\circ} 3$ ).

4) Armature de flèche tranchante triangulaire sur éclat mince; la retouche s'est effectuée comme sur la pièce précédente: d'abord la face inférieure, puis la face supérieure; dimensions: $23 \mathrm{~mm}$ x $16 \mathrm{~mm}$ x $3 \mathrm{~mm}$ (fig. 23, $\mathrm{n}^{\circ} 4$ ).

5) Pic-ciseau; il s'agit d'un exemplaire comparable à celui trouvé dans la sépulture 2, mais de dimensions plus modestes; la retouche est bifaciale couvrante; des enlèvements dans l'axe de la pièce ont permis d'aménager le biseau; dimensions: $76 \mathrm{~mm}$ x $20 \mathrm{~mm}$ x $15 \mathrm{~mm}$ (fig. 23, $\mathrm{n}^{\circ} 5$ ).

6) Éclat partiellement cortical par percussion dure; dimensions: $53 \mathrm{~mm}$ x $34 \mathrm{~mm}$ x $15 \mathrm{~mm}$.

7) Fragment mésial d'éclat sans cortex; dimensions: $48 \mathrm{~mm} \times 23 \mathrm{~mm} \times 7 \mathrm{~mm}$.

8) Fragment distal d'éclat remontant sur le précédent (cassure ancienne par flexion).

9) Éclat de quartz (verre opaque à fissures internes) ; les stigmates du débitage sont difficiles à observer, mais il 

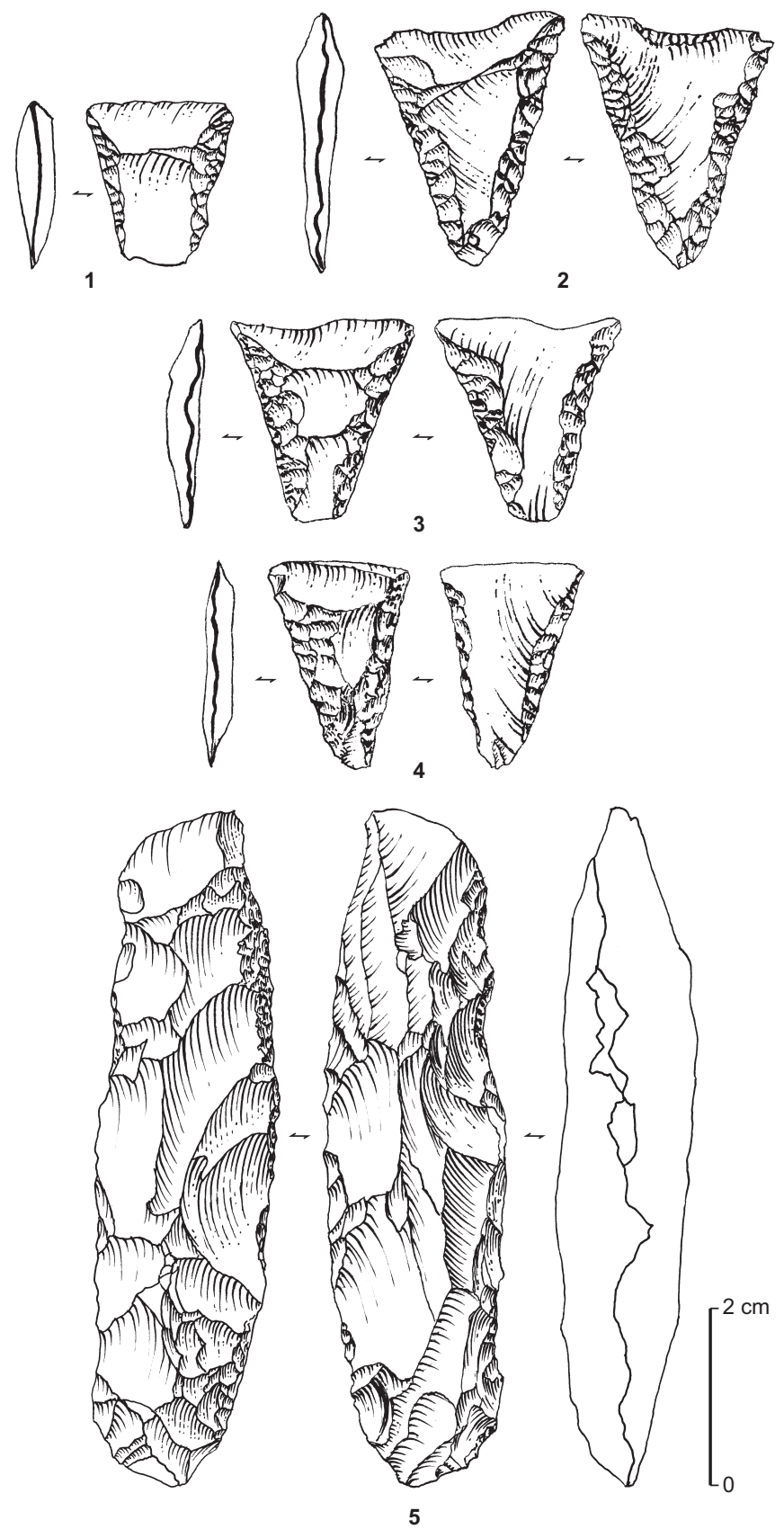

Fig. 23 - Mobilier lithique de la sépulture 5: 1-4, armatures de flèche; 5, pic-ciseau. L'analogie entre le mobilier des sépultures 2 et 5 est frappante (dessin: D. Molez, CNRS).

semble qu'une esquille bulbaire soit discernable; dimensions: $14 \mathrm{~mm} \times 16 \mathrm{~mm}$ x $6 \mathrm{~mm}$.

Sept des pièces sont au même niveau que l'enfant inhumé. Le dépôt funéraire est, comme dans les précédentes sépultures, uniquement lithique. Les quatre armatures sont près de la tête, empilées les unes sur les autres, partie active vers le nord, l'emplacement des hampes pouvant alors se trouver le long de la partie supérieure du corps; l'une des armatures a toutefois légèrement basculé comme dans un espace vide, ce qui serait un élément en faveur de la présence d'un carquois. La relation des autres pièces avec l'inhumé est plus problématique: pour le pic-ciseau et l'éclat 7 avec son extrémité 8 , la destruction des ossements ne permet pas de déterminer si les pièces reposaient sous ou sur le défunt. Dans le cas du pic-ciseau, miniaturisé et comme à la taille de l'enfant inhumé, c'est l'analogie avec la sépulture 2 qui permet d'envisager le dépôt d'un outil emmanché sur l'avant-bras. Quant à l'éclat par percussion dure et à l'éclat de quartz (6 et 9 ), ils proviendraient du remplissage.

\section{SÉPULTURE 6}

La sépulture 6 reprend l'alignement sud-est/nord-ouest observé pour S1, S2, S3 et S4. Le remplissage de la fosse est assez voisin de celui des autres sépultures, sa forme est similaire et sa profondeur presque identique. Toutefois cette sépulture a été perturbée par des labours profonds qui ont atteint ici $0,32 \mathrm{~m}$ sous la surface (fig. 24). La fosse n'est donc plus profonde que de $0,16 \mathrm{~m}$ sous le décapage et présente la forme d'une cuvette irrégulière: si au niveau $-0,37 \mathrm{~m}$ elle mesure 2,30 m x 1,10 m, elle fait $2,05 \mathrm{~m} \times 0,95 \mathrm{~m}, 5 \mathrm{~cm}$ plus bas.

Son remplissage est aussi moins fin et moins foncé. Sept pierres agencées en arc de cercle forment un dais au niveau du bloc craniofacial. Deux pierres ont encore été retrouvées sur la poitrine et un galet sphérique entre les talons.

La sépulture 6 correspond à l'inhumation individuelle d'un sujet adulte (fig. 25). Il repose en position étendue sur le dos, la tête au nord-ouest et les pieds au sud-est. La position de la tête n'est pas clairement discernable (face antérieure ou latérale droite). Les membres sont étendus dans l'axe du corps. Les membres supérieurs reposent le long du tronc, la main gauche sur la hanche, la main droite en pronation à côté de la hanche. Les membres inférieurs sont parallèles, les pieds contraints.

Deux lames de silex ont été retrouvées symétriquement, au-dessus de l'épaule droite et sous l'épaule gauche.

\section{CONSERVATION ET PERTURBATIONS}

L'état des restes osseux est variable d'une région anatomique à l'autre. La tête osseuse est fragmentée et lacunaire: les vestiges les plus hauts étaient érodés; la branche gauche 


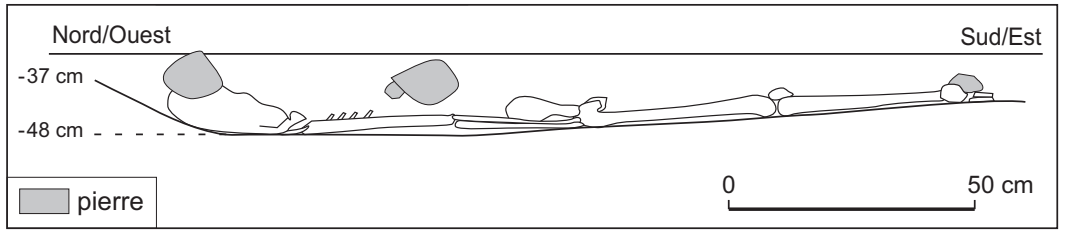

Fig. 24 - Coupe longitudinale de la sépulture 6. Les labours ont fortement entamé la sépulture (relevé: P. Chambon, CNRS et J.-P. Delor).

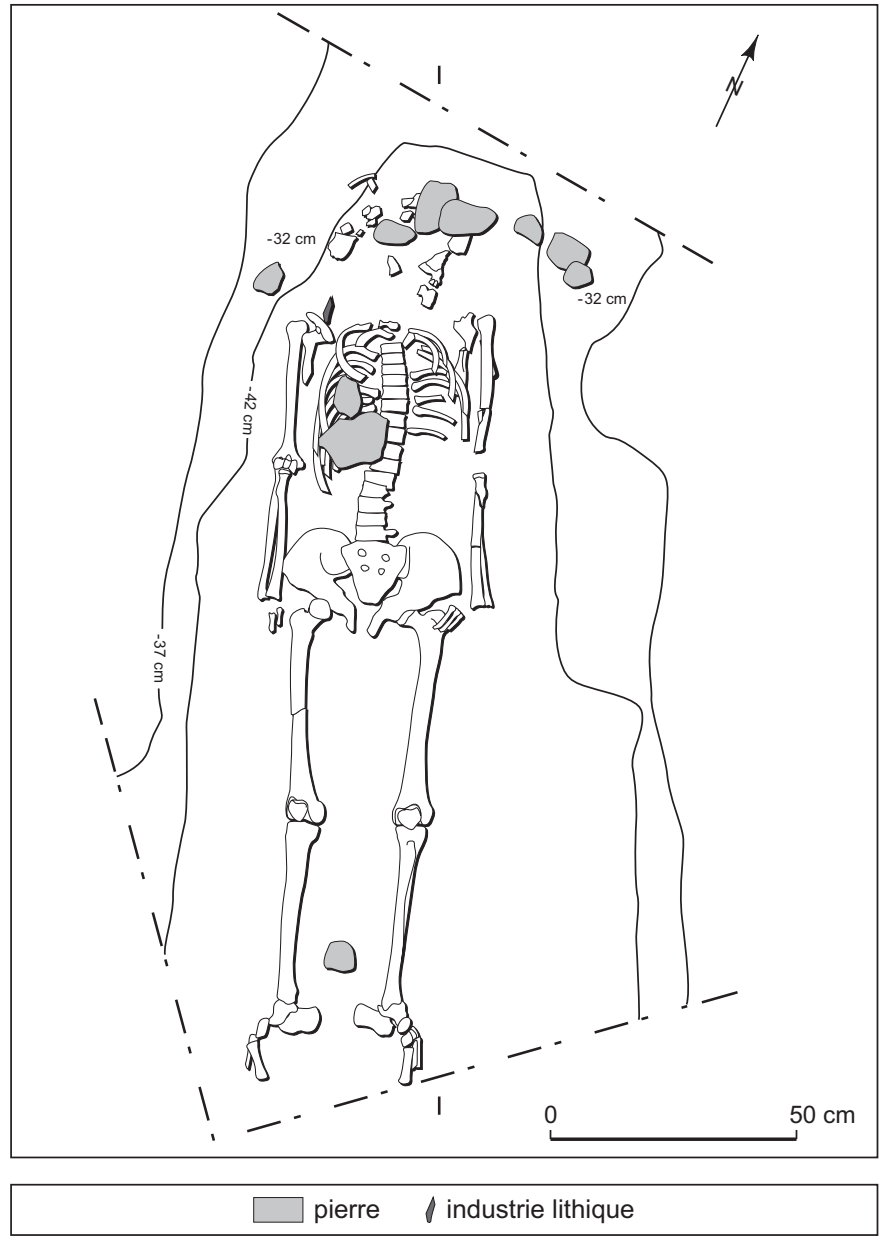

Fig. 25 - Plan de la sépulture 6 (relevé: P. Chambon, CNRS).

de la mandibule se réduit à quelques esquilles. La colonne cervicale n'apparaît pas sur les photographies mais des fragments correspondant au moins à six d'entre elles figurent à l'inventaire. Bien qu'érodé, le reste de la colonne est également représenté: les corps vertébraux apparaissent bien sur les photographies. Les côtes sont inégalement conservées: leur état est meilleur du côté droit que du côté gauche et de moins en moins bon du haut en bas du thorax. Les deux clavicules, invisibles sur les clichés, font partie des restes que nous avons examinés. Les os coxaux sont érodés. Les os des membres sont dans l'ensemble bien représentés, avec des variations: les membres supérieurs sont fragmentés et les épiphyses sont assez mal conservées; les mains ne sont figurées que par une poignée de métacarpiens; les membres inférieurs sont les ossements les mieux préservés de la sépulture, ainsi que les pieds.

Au sein de la sépulture 6 , la conservation défectueuse explique sans doute les lacunes de l'inventaire. Il manque essentiellement des fragments d'os, principalement parmi les os plats, et des petites pièces des extrémités. En revanche, il est plus difficile d'expliquer pourquoi l'étage cervical de la colonne vertébrale, le haut du thorax et la ceinture des membres supérieurs sont absents ou fragmentaires sur le relevé initial et les photographiques. Le rapport de 1983 signale enfin que la sépulture fut repérée par la présence d'un fragment osseux remonté par les labours, et attribue à ces mêmes labours le bouleversement du bloc craniofacial (Delor, 1983). Est-ce la cause de ces lacunes, ou faut-il conclure qu'un animal a bouleversé cette partie du squelette, ou bien n'est-ce finalement qu'un effet de fouille?

\section{CONNEXIONS ET DISLOCATIONS}

Si on fait abstraction des perturbations de sa partie supérieure, le squelette a conservé une forte cohérence anatomique.

L'emboîtement des vertèbres, en face antérieure, est régulier de la première vertèbre thoracique jusqu'au sacrum. Les côtes se sont logiquement affaissées dans le volume initial du thorax.

La tête de l'humérus gauche est en vis-à-vis de la cavité glénoïdale. L'extrémité distale de l'humérus comme la majeure partie de l'ulna ne sont pas visibles sur les photographies, néanmoins la situation du radius et de l'extrémité distale de l'ulna n'implique aucune dislocation. En revanche, la position des trois métacarpiens sur la hanche n'est pas compatible avec celle de l'avant-bras: le métacarpe a basculé alors qu'il se trouvait de chant. Du côté droit, la tête de l'humérus paraît plus haute que la scapula (l'épaule est-elle haussée?); la partie libre du membre est en connexion, dans une position stable. 
Le bassin s'est totalement ouvert: les ailes des iliums reposent à plat sur le sol. Le membre inférieur gauche est en connexion depuis la hanche jusqu'au genou, en face antérieure. L'articulation de la cheville est rompue: le pied est en vue médiale. Il offre une bonne cohérence générale mais les seuls liens stricts préservés lient des pièces d'un même «bloc»: tarse postérieur, cunéiformes entre eux, métatarsiens 2 et 3 . Du côté droit, le fémur a légèrement pivoté latéralement et apparaît en vue antéromédiale (il n'est pas possible de déterminer si la tête du fémur est toujours logée dans l'acétabulum), le tibia a poursuivi la rotation et est visible par sa face médiale; la patella est restée sur les condyles du fémur. Le pied droit présente une situation comparable au gauche mais, de ce côté, les liens sont plus distendus. Les photographies montrent la dislocation du cunéiforme médial (?) et celle du métatarsien 1.

\section{MILIEU DE DÉCOMPOSITION ET TYPE DE STRUCTURE}

Les articulations en connexion sont nombreuses: colonne vertébrale, membre supérieur droit, membres inférieurs. Cependant elles mettent souvent en jeu des pièces que la décomposition a laissé dans une position d'équilibre. C'est notamment le cas des vertèbres, et des membres supérieurs. Le cas des membres inférieurs est différent. La rotation du membre inférieur suite à la rupture de la hanche est un phénomène classique. Elle s'est ici opérée uniquement du côté droit et incomplètement; en outre, la patella est à sa place. Enfin, le pied gauche est partiellement disloqué, mais cette dislocation ne se traduit pas par une mise à plat des ossements (les photographies ne sont pas claires sur ce point). Les dislocations sont plus discrètes, mais elles s'accompagnent de la transgression du volume corporel: citons la main gauche, l'ouverture du bassin, le pied droit. Ces indices ténus nous conduisent à préférer la thèse d'une décomposition en espace vide.

Une hypothèse complémentaire est cependant nécessaire pour traduire le maintien des membres inférieurs, notamment de la patella droite. Une aide précieuse nous est ici fournie par la disposition particulière des pieds, le métatarse perpendiculaire au tarse postérieur, dans l'axe du corps. Corrélée au parallélisme des membres inférieurs, cette disposition pourrait témoigner de l'existence de montants de part et d'autre. Aucun élément ne permet, en l'état, de prolonger ces effets de parois au-delà des hanches.

Les faits sont insuffisants pour conclure sur le type de structure. Signalons néanmoins que nous avons déjà reconnu, dans des nécropoles contemporaines, des effets de parois limités aux membres inférieurs. Dans les cas les plus favorables, nous avons pu conclure que le sujet reposait dans un contenant à rebords bas, sur lesquels les membres supérieurs prenaient appui (Chambon et al., 2009).

\section{L'INDIVIDU}

Conservation osseuse: à l'exception de la tête osseuse très partiellement représentée, le squelette 6 présente une bonne conservation et une faible fragmentation.

Âge: la maturation complète de l'extrémité sternale de la clavicule et de la crête iliaque plaide en faveur d'un adulte de plus de 25 ans. L'analyse de ses surfaces sacro-pelviennes nous indique un âge ne dépassant pas les 40 ans.

Sexe: les trois seuls critères morphoscopiques observables sur les os coxaux (région préauriculaire, grande incisure ischiatique et arc composé) décrivent un sujet masculin, tel qu'il avait été identifié en 1983. Ce résultat est confirmé par la DSP. La morphologie crânienne identifiée comme masculine d'après les cinq critères observés est donc cohérente avec les résultats obtenus sur les os du bassin.

Stature: la longueur du fémur nous indique une stature d'environ 1,63 m.

État buccodentaire: vingt-six dents sont conservées (un tiers d'entre elles sont isolées). L'usure est homogène sur l'ensemble des arcades, atteignant un stade de sévérité relativement avancé : les cuspides sont largement arasées, les îlots dentinaires se rejoignent, mais un pourtour d'émail persiste dans chacun des cas. La sévérité de l'usure est nettement plus prononcée sur les deux premières molaires supérieures. En revanche, la troisième molaire supérieure gauche n'est que légèrement atteinte. Notons la résorption alvéolaire complète observée sur le fragment de l'hémimaxillaire gauche au niveau de la deuxième molaire supérieure, témoignant de sa perte ante mortem. La surface de l'os présente à ce niveau une réaction périostée. L’usure dentaire générale, bien que relativement avancée, et la faible parodontose de l'ensemble des dents observées in situ ne paraissent pas cohérentes avec la chute de cette dent. Une importante lésion carieuse pourrait être à l'origine de cette perte, bien qu'aucune carie n'ait été décelée sur les faces de contact des deux dents conjointes. L'extraction volontaire de cette dent pourrait être envisagée mais cette hypothèse reste à approfondir. Le dépôt de tartre est léger, non systématique et n'atteint que le collet des dents concernées.

Indicateurs de stress biologique: six dents (incisives et canines supérieures et inférieures) présentent au moins 
une hypoplasie linéaire de l'émail dentaire. Toutes ont été identifiées au niveau du tiers moyen de la couronne. La sévérité des atteintes est plus prononcée sur les deux canines inférieures.

\section{LE MOBILIER}

1) Éclat mince et laminaire dont le talon est invisible; dimensions: $47 \mathrm{~mm}$ x $10 \mathrm{~mm} \times 1 \mathrm{~mm}$.

2) Lame par percussion tendre (talon facetté et mince, corniche abrasée, présence d'une lèvre) à extrémité rebroussée, deux pans, sens de débitage unidirectionnel; elle porte une retouche marginale irrégulière sur les deux bords; dimensions: $56 \mathrm{~mm}$ x $19 \mathrm{~mm}$ x $4 \mathrm{~mm}$.

Le premier éclat est disposé au niveau de l'épaule droite alors que la lame se trouve sous son omoplate gauche. C'est la troisième fois que l'on rencontre cet agencement à Chichery (voir sépultures 2 et 3), un dispositif de portage sur l'épaule, dans un sac ou une poche, peut être envisagé.

\section{FOSSE F3}

La fosse F3 complète ce premier groupe de structures. Il s'agit d'un calage de poteau, semblable à F2. L'excavation en entonnoir est ici plus large en surface mais présente un surcreusement qui n'excède pas $14 \mathrm{~cm}$ de diamètre au niveau $-0,85 \mathrm{~m}$. Soixante-cinq pierres (granite, quartzite, calcaire et silex) proviennent, là encore, de la terrasse alluviale. Elles ont été placées en lits successifs mais ne révèlent pas le fantôme d'un poteau qu'on attend mais qui a pu être récupéré ultérieurement par basculement, opération qui a pu bouleverser l'agencement du blocage. Le remplissage comportait en outre un petit nucléus polyédrique.

\section{SÉPULTURE 7}

La sépulture 7 présente une forme elliptique régulière et caractéristique se dessinant bien sur le gravier; elle mesure $1,85 \mathrm{~m}$ × $0,90 \mathrm{~m}$, pratiquement à la taille de l'individu qu'elle contient (fig. 26). Elle est orientée nord-sud avec une légère inclinaison de $2^{\circ}$ vers l'est. Son remplissage est constitué d'une terre fine foncée, exempte de graviers. Les bords sont initialement verticaux puis s'arrondissent vers le fond presque horizontal, à - 0,44 $\mathrm{m}$ à la tête et - 0,47 $\mathrm{m}$ aux pieds (sous le décapage).

Le sujet repose en position étendue sur le dos dans le grand axe de la fosse, la tête au sud et les pieds au nord (fig. 27). Il est décentré: distant de $0,10 \mathrm{~m}$ de la paroi est,

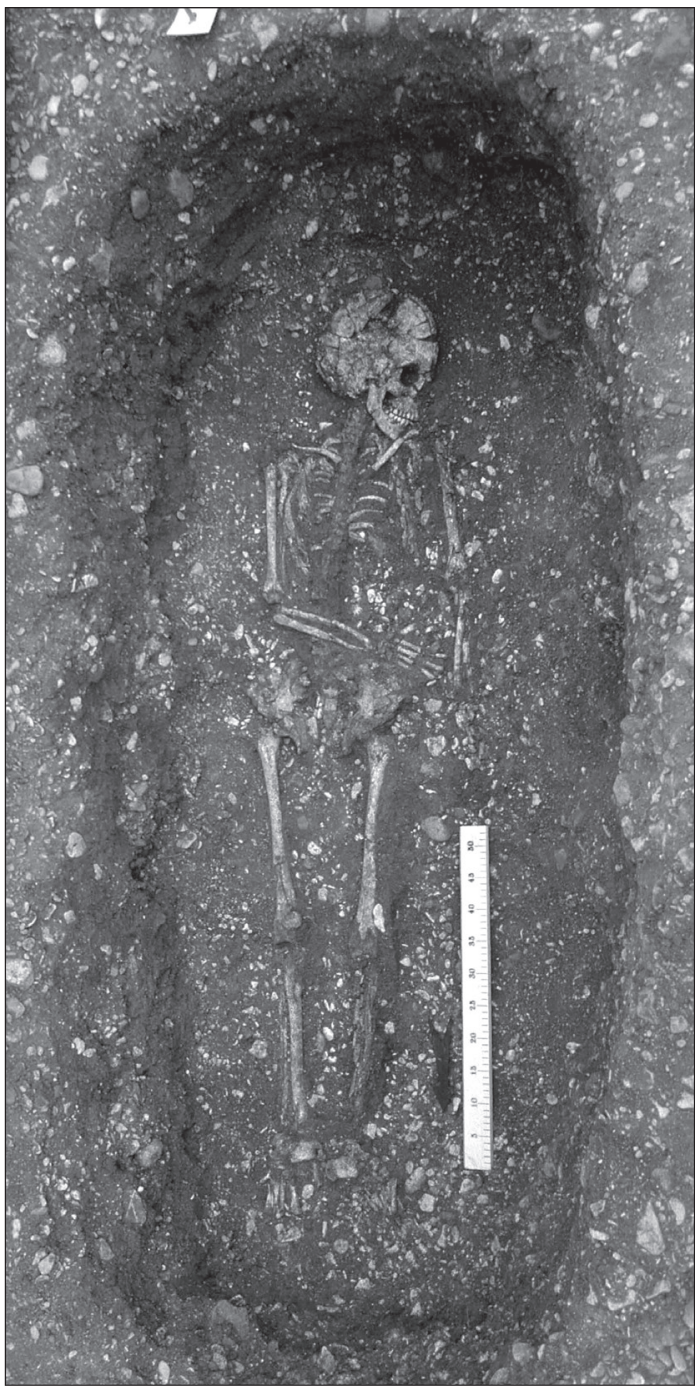

Fig. 26 - Vue générale de la sépulture 7 (cliché:J.-P. Delor).

alors que l'écart le séparant de la paroi ouest fluctue de $0,35 \mathrm{~m}$ à plus de $0,50 \mathrm{~m}$. Sa tête est tournée vers l'épaule gauche, le regard est dirigé vers le nord-ouest. La bouche est fermée.

Le membre supérieur gauche est étendu mais légèrement écarté du tronc; la main est en pronation. Le membre supérieur droit est fléchi, l'avant-bras sur le ventre, la main au-dessus de la hanche gauche, la paume vers le sol. Les membres inférieurs sont étendus. La cheville gauche est en position de repos. Le pied droit est en extension (incomplète).

Sur le côté droit du squelette, les ossements s'alignent: un effet de paroi court de l'humérus au pied. 


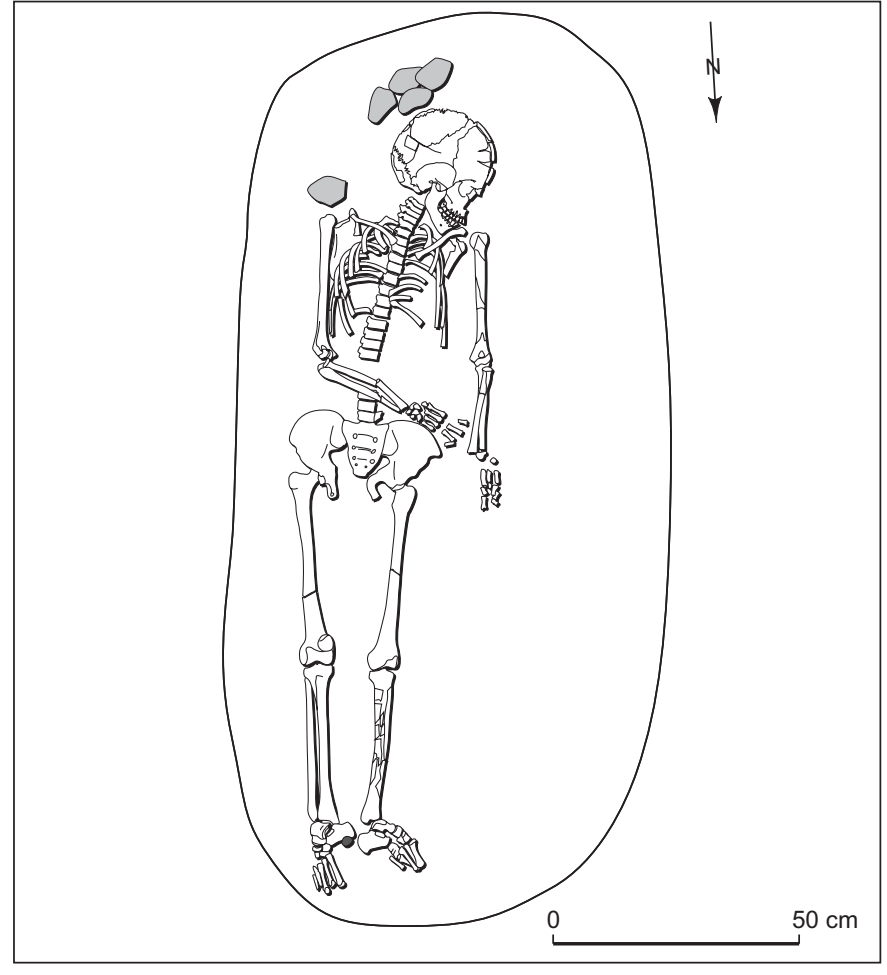

$\square$ pierre • rhynchonelle

Fig. 27 - Plan de la sépulture 7 (relevé: P. Chambon, CNRS).

Cendres et charbons de bois appartenant à une pièce de bois longue d'environ $0,30 \mathrm{~m}$ ont été observés dans le remplissage au sud (fig. 28). Quatre fragments d'éclats et de lames en silex et un tesson atypique épars, de même que cinq petites pierres situées à proximité du bloc craniofacial, ont été recueillis ${ }^{12}$.

\section{CONSERVATION ET PERTURBATIONS}

L'état des ossements est assez moyen. Les épiphyses sont en mauvais état: c'est notamment le cas des têtes humérales ou des extrémités des tibias. Les corps vertébraux sont peu visibles sur les photographies après le milieu de la colonne thoracique, et en laboratoire nous n'en avons inventorié que des fragments depuis la vertèbre thoracique 3 jusqu'à la vertèbre lombaire 5 . Les os des tarses antérieurs ont des contours indistincts. La désagrégation des pièces les plus fragiles du squelette est au final très vraisemblable.

12. Nous n'avons pas retrouvé ces artefacts.
Aucun terrier n'a été repéré à la fouille. Les manques ponctuels traduisent un déficit de conservation. L'absence de la partie inférieure du thorax, côté gauche, correspond sans doute à l'impossibilité de dégager des côtes réduites à de simples traces ou à des agrégats d'esquilles. Enfin, la position des vestiges osseux ne montre pas d'incohérence anatomique et résulte du processus de décomposition.

\section{CONNEXIONS ET DISLOCATIONS}

L'observation des relations articulaires a été réalisée d'après les photographies. Elle reste forcément incomplète, mais suffit au diagnostic.

Le bloc craniofacial et la mandibule sont en connexion, bouche fermée. Nous ne pouvons distinguer les trois premières vertèbres cervicales, sans doute présentes dans leur zone d'origine. Il est difficile de distinguer l'enchaînement des vertèbres, après les premières vertèbres thoraciques, toutefois il ne semble pas exister de hiatus ou de distension avant le segment lombaire. À l'autre extrémité, les trois dernières vertèbres lombaires sont liées entre elles et au sacrum. Sous le radius droit et immédiatement au nord de celui-ci, aucune vertèbre n'apparaît sur les photographies: d'après notre décompte, il s'agit d'une absence réelle, c'està-dire d'une rupture d'environ $6 \mathrm{~cm}$ entre la deuxième et la troisième vertèbre lombaire ${ }^{13}$. Les côtes repérées se sont logiquement effondrées dans le volume thoracique.

Si la cohérence des membres supérieurs est respectée, leur examen révèle diverses dislocations. Du côté gauche, il est impossible d'apprécier la liaison entre la scapula et l'humérus. Les trois principaux os longs sont en connexion (l'extrémité proximale du radius est à peine décalée latéralement par rapport au capitulum); en revanche, les éléments de la main mis au jour sont désaxés vers l'ouest par rapport à l'extrémité de l'avant-bras. La position oblique de la scapula droite comme la dislocation du coude suggèrent que l'humérus, encore surélevé, chevauchait initialement en partie les côtes. L'avant-bras est fracturé sur la colonne vertébrale. Le carpe semble essentiellement en connexion, ainsi que le métacarpe et les phalanges proximales 2 à 4 (si nous les avons correctement identifiées, les phalanges proximales 1 et 5 sont écartées de leur position anatomique).

Le bassin s'est largement ouvert: seule l'aile de l'ilium droit reste très légèrement oblique. Les têtes des fémurs

13. L'écart entre les coudes et le bassin apporte une confirmation indirecte de cette rupture: alors que l'épaule gauche n'est pas haussée et que le membre supérieur est étiré le long du corps, le bout des doigts se trouve à peine au niveau de la hanche. 


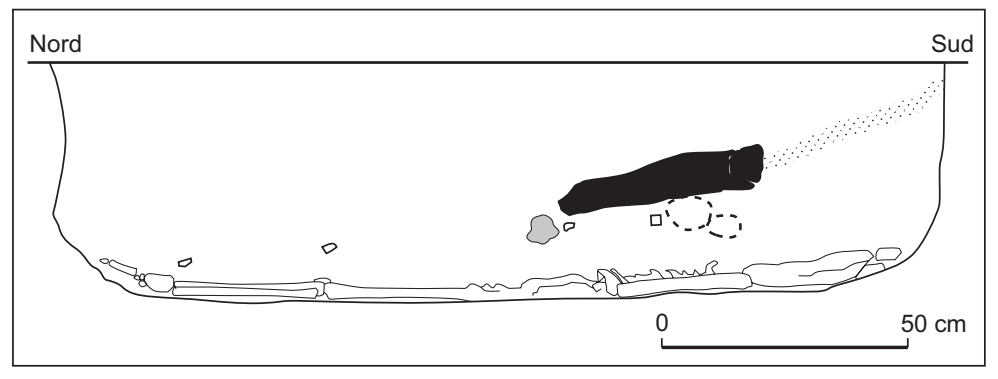

pierre $\quad$ tesson $\quad \square$ cendres charbon

a

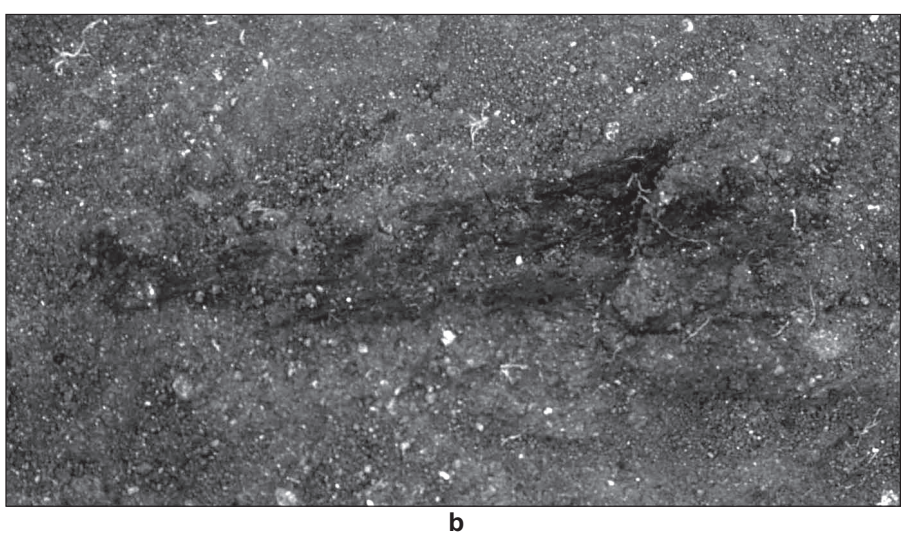

Fig. 28 - a, coupe longitudinale de la sépulture 7; $\boldsymbol{b}$, le fragment de bois carbonisé appartient-il à la structure initiale? (relevé: P. Chambon, CNRS et J.-P. Delor; cliché:J.-P. Delor). sont toujours logées dans les acétabulums (on ne peut pas le certifier du côté droit) et ces os sont demeurés en face antérieure. Le tibia gauche est très fragmenté, et on peut seulement présumer une vue médiale; la fibula se trouve sans doute sous le tibia. L'extrémité proximale du tibia droit est plus basse que l'épiphyse contiguë du fémur; par rapport à son symétrique, il n'a que peu tourné et se présente en vue antéromédiale. La fibula est visible à son côté mais devrait normalement être légèrement masquée. La patella droite, la seule en place, a glissé de quelques centimètres en direction de l'extrémité proximale du fémur. Les articulations des chevilles sont rompues. À gauche, le tarse postérieur est en connexion, en vue médiale. L'os naviculaire semble en contact avec le talus, mais les liens sont distendus avec les cunéiformes. L'os cuboïde apparaît essentiellement en face inférieure, nettement séparé des cunéiformes. Les métatarsiens sont disloqués, les extrémités proximales des métatarsiens 3 et 4 chevauchent l'os cuboïde, le métatarsien 5 est en retrait, les parties distales des métatarsiens se croisent. Le pied droit ne présente aucun lien strict; sa position décrit un arc de cercle, du sud-ouest au nord-ouest. Le calcanéus est en vue médiale, mais la face d'apparition du talus comprend une forte composante supérieure. Le tarse antérieur s'est affaissé en avant du talus, l'os naviculaire basculé sur les cunéiformes. Le métatarse gît à plat sur le sol.

\section{MILIEU DE DÉCOMPOSITION ET TYPE DE STRUCTURE}

Le sujet s'est décomposé dans un espace vide. Parmi les principales articulations disloquées, nous citerons la colonne vertébrale, l'épaule droite, le coude droit, le poignet gauche, le bassin, les pieds... Toutes ces ruptures se traduisent par une transgression du volume initial du cadavre; les ossements, libérés des téguments, se sont affaissés jusqu'à une nouvelle situation d'équilibre. Inversement, les articulations demeurées en connexion mettent en jeu des ossements en position stable (le cas des fémurs reste en suspens).

Un os apparaît toutefois dans une position instable (au sein d'un espace vide): l'humérus droit, disjoint de l'épaule et du coude, devrait apparaître en face antérieure, la palette humérale à plat sur le sol; en effet, sa situation en face antérolatérale impliquait un déséquilibre à l'issue de la décomposition; or son affaissement latéral a été stoppé. C'est encore côté droit que nous avons noté un effet de 
paroi, les ossements s'alignant depuis l'humérus jusqu'au pied. L'arc de cercle imprimé au pied est significatif: le métatarse est renvoyé en deçà de la limite virtuelle que constitue l'effet de paroi. Relier ce témoignage avec la décomposition en espace vide est logique.

La décomposition en espace vide ne suffit pas à expliquer la rupture intervenue dans la partie inférieure de la colonne vertébrale. Cet écartement n'a induit aucune rupture secondaire tant dans la moitié supérieure que dans la moitié inférieure du squelette. La dislocation ne correspond pas à un déplacement d'une ou deux vertèbres mais bien au glissement complet de l'une des deux parties du squelette. Seule la rupture d'un fond, telle que l'ont décrite H. Duday, F. Lambach et S. Plouin (1990) pour une sépulture de Nordhouse dans le Bas-Rhin, peut traduire un tel phénomène.

Un effet de paroi fortement marqué du côté droit (de l'épaule au pied), une attitude initiale où le corps, en appui contre une paroi, est légèrement tourné sur la gauche, un dépôt décentré dans la fosse, voilà trois arguments supplémentaires en faveur d'un contenant mobile (Chambon, Lanchon, 2003). Le sujet était donc inclus dans un contenant rigide mobile.

On ne peut exclure une architecture à même la fosse, en plus de ce contenant. Aucun témoignage n'étaye ou n'invalide cette hypothèse.

\section{L'INDIVIDU}

Conservation osseuse: la tête osseuse de l'individu 7 est bien conservée, la mandibule et l'os maxillaire sont complets. Le squelette infracrânien est en revanche inégalement représenté et souffre d'une fragmentation assez importante.

Âge: le sujet a été identifié en 1984 comme un jeune adulte (Delor, 1984). Notre diagnostic le rajeunit légèrement, un certain nombre d'éléments osseux témoignant d'une maturation incomplète: la synchondrose sphénooccipitale n'est pas soudée, les condyles de la mandibule gardent la trace des cartilages de croissance, le listel des corps vertébraux est en cours de synostose, la tubérosité ischiatique reste indépendante, l'extrémité distale du fémur gauche, la tubérosité des calcanéus et l'épiphyse proximale du premier métatarsien présentent encore une trace de suture. D'après différents auteurs, cet état de maturation générale traduirait un état pubère (Olivier, 1960; Birkner, 1980; Kamina, Zwobada-Kamina, 1983). Nous classons donc l'individu 7 parmi les [15-19] ans.
Sexe: le sujet a été identifié en 1984 comme un homme. La détermination du sexe des adolescents postpubères peut dans certains cas être appliquée, dans la mesure où les trois éléments de l'os coxal (ilium, ischium et pubis) sont parfaitement soudés et que les mesures prises sur l'os excluent celles comprenant un des derniers éléments non matures (crête iliaque, épine et tubérosité ischiatiques, épine iliaque antéro-inférieure). Cette démarche reste toutefois problématique lorsque le résultat obtenu est celui d'un individu masculin. Il est dès lors impossible de savoir s'il s'agit effectivement d'un homme ou d'une femme qui n'aurait pas encore subi le remodelage de son bassin sous l'influence hormonale.

Dans notre cas, les mesures prises sur les os coxaux sont insuffisantes pour permettre une diagnose probabiliste. Les quelques critères morphologiques observables tendent vers une attribution masculine, expliquant peut-être le diagnostic établi en 1984. Toutefois, ces observations sont insuffisantes pour nous permettre de conclure (deux critères sexés sur cinq). En l'état, et compte tenu des observations faites sur les limites d'une attribution sexuelle sur grand adolescent, aucune diagnose sexuelle ne sera réalisée sur l'individu 7.

État buccodentaire: l'ensemble des dents est observable in situ. L'usure générale est faible à nulle. Nous notons de légères caries sur la face occlusale des première et deuxième molaires inférieures gauches. Aucune trace de maladie parodontale n'est constatée. Le dépôt de tarte atteint préférentiellement la denture inférieure, sur la face linguale des couronnes.

Indicateurs de stress biologique: trois des quatre canines présentent une légère hypoplasie linéaire de l'émail dentaire, localisée au niveau du tiers moyen de la couronne.

\section{SÉPULTURE 8}

La sépulture 8 se présentait au décapage comme une fosse de forme irrégulière mesurant $2 \mathrm{~m} \mathrm{x} 1,25 \mathrm{~m}$, dont l'axe dominant correspond au nord-ouest/sud-est. La tombe prend une forme elliptique plus conventionnelle à 0,47 m sous le décapage: elle mesure alors $1,40 \mathrm{~m}$ x 0,55 m et son orientation adopte un axe est-ouest avec un décalage de $3^{\circ}$ vers le nord. Indépendamment des diverticules, les bords de la fosse sont sensiblement verticaux puis s'arrondissent vers le fond qui est atteint à - 0,52 m. Manifestement la sépulture a été perturbée par des animaux fouisseurs. On observe une coulée de traces d'origine organique (fig. 29). Le remplissage, très homogène, est constitué de terre fine 


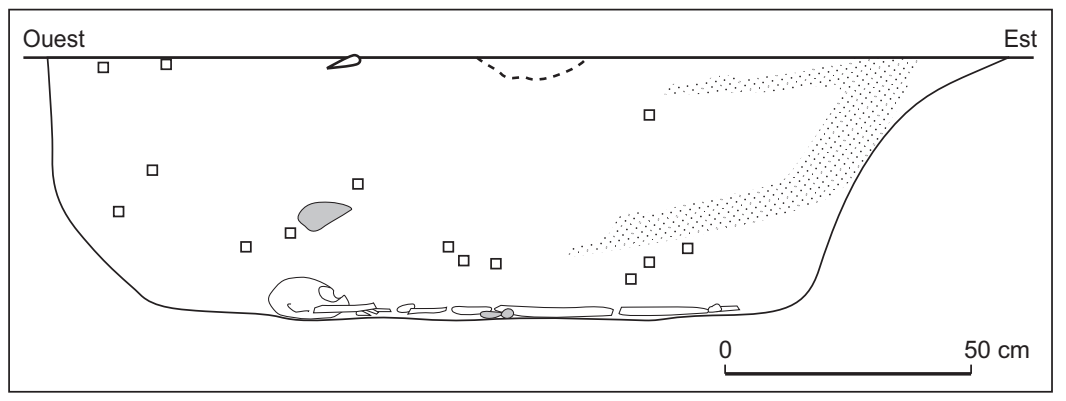

Fig. 29 - Coupe longitudinale de la sépulture 8 (relevé: P. Chambon, CNRS et J.-P. Delor).

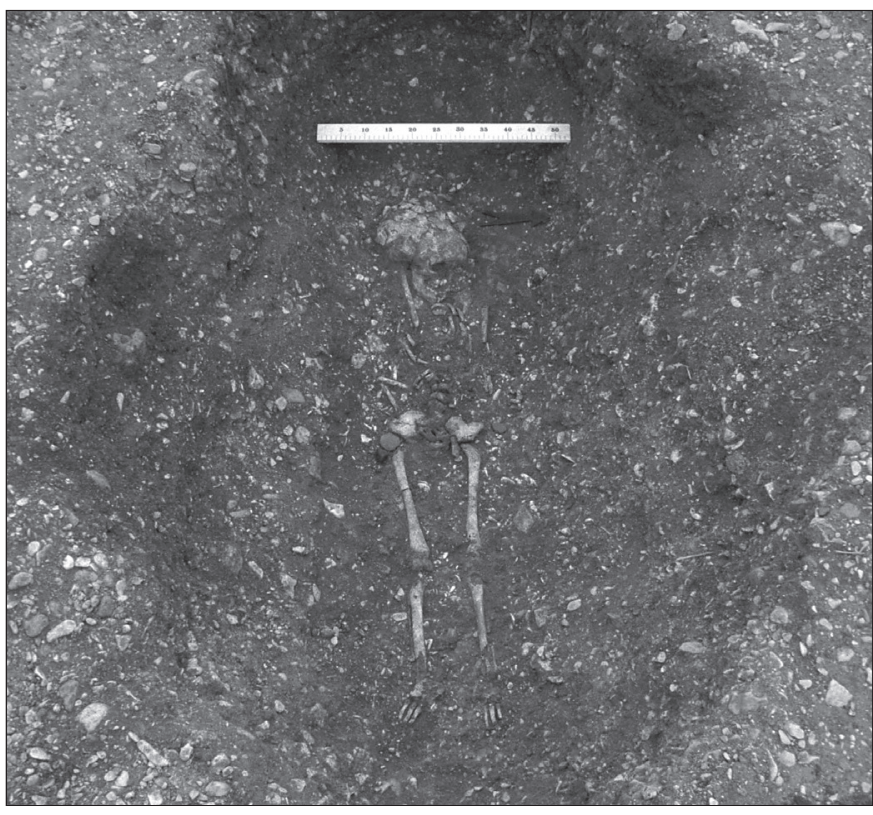

Fig. 30 - Vue générale de la sépulture 8 (cliché:J.-P. Delor).

sombre. Il contient quelques éléments lithiques et 48 petits tessons. Il s'agit de céramique sans décor. Ces vestiges sont répartis autour du remplissage de la sépulture elle-même, à l'exclusion des diverticules.

La tombe a livré les restes d'un sujet immature, inhumé en position étendue sur le dos, la tête à l'ouest et les pieds à l'est (fig. 30 et 31). La tête est redressée et légèrement tournée vers la gauche, la bouche est fermée. Le membre supérieur gauche était sans doute étendu le long du tronc; le droit est fléchi (coude écarté?), la main sur le ventre. Les membres inférieurs sont étendus parallèlement, dans l'axe du corps. Du côté gauche, l'humérus, l'épine iliaque antérosupérieure, le fémur, le tibia et un métatarsien (le 4?) sont situés sur une même droite.

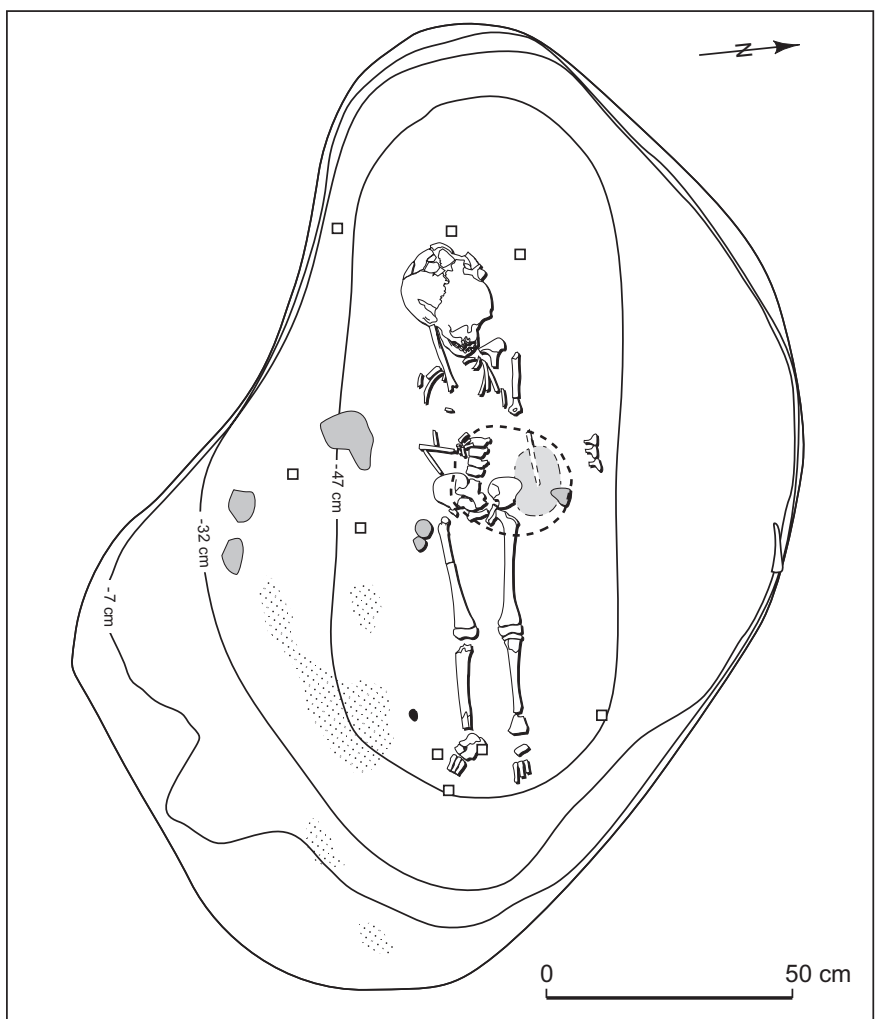

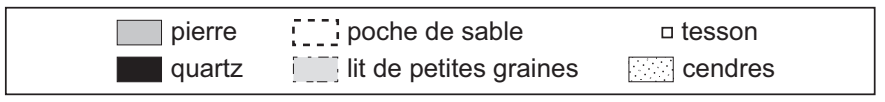

Fig. 31 - Plan la sépulture 8. Le creusement n'est régulier que dans sa partie inférieure (relevé: $P$. Chambon, CNRS).

\section{CONSERVATION ET PERTURBATIONS}

L'état des ossements est moyen. Les photographies révèlent des ossements érodés et fragmentés. Les seules épiphyses indiscutablement présentes sont les condyles des fémurs et ceux du tibia gauche. L'inventaire des restes osseux est très incomplet. La colonne vertébrale est figurée, 
pour l'étage cervical, par l'axis et un demi-arc de la vertèbre cervicale 7, pour les étages thoracique et lombaire, par une poignée d'arcs et une dizaine de corps vertébraux. Le thorax se réduit à quelques portions de côtes. On ne distingue de la ceinture des membres supérieurs qu'un morceau de la scapula gauche. L'un des deux os de l'avant-bras gauche manque, ainsi que toute la main.

Dans la partie inférieure du squelette, on note encore l'absence des patellas, des fibulas (à moins qu'elles soient masquées sur les photographies) et d'une partie des pieds.

Si les déficits de l'inventaire correspondent essentiellement aux pièces les plus fragiles du squelette (avec une faible épaisseur d'os compact), la mauvaise conservation n'explique pas la disparition d'une partie de la ceinture des membres supérieurs et de la moitié de l'avant-bras gauche. De fait, c'est la partie supérieure du tronc qui affiche les manques les plus «suspects». Le plan comme les observations de terrain lèvent l'incertitude: une solution de continuité large d'une dizaine de centimètres sépare le haut du bas du tronc; l'humérus a migré vers le bloc craniofacial, son extrémité proximale se trouvant sous l'os occipital; trois processus vertébraux ont été mis au jour contre la paroi, à près de $0,25 \mathrm{~m}$ de leur emplacement anatomique. De telles perturbations sont la signature d'un animal fouisseur. Son théâtre d'opération fut essentiellement le thorax: nous lui sommes redevables de l'absence des côtes et d'une partie de la colonne; nous lui accorderons la position de l'humérus au bénéfice du doute, malgré l'absence apparente de perturbation à la base du bloc craniofacial. Peut-on néanmoins expliquer par ce biais les dislocations les plus frappantes, telle la disjonction de l'avant-bras droit? Ses galeries étaient suffisamment larges pour permettre le déplacement de plusieurs vertèbres en bloc. Son trafic fut suffisamment intense pour entraîner la destruction des vertèbres.

\section{CONNEXIONS ET DISLOCATIONS}

L'examen des liens articulaires, réalisé d'après la documentation photographique, est sévèrement limité par les perturbations.

Le bloc craniofacial et la mandibule sont en connexion. De la colonne vertébrale ne subsiste que la suite anatomique des vertèbres lombaires 2 à 5 .

Le fragment de scapula gauche mis au jour paraît bien écarté de l'humérus. Celui-ci ne se trouvait pas, semble-t-il, sur le trajet du fouisseur: il repose en position stable, en vue antérieure. Les deux os de l'avant-bras droit, demeurés en contact à leur extrémité proximale, divergent de $50^{\circ}$; c'est l'ulna qui témoigne de la situation initiale de l'avant-bras. Les métacarpiens et les phalanges visibles ont une disposition cohérente, mais sans réelle connexion.

Les deux iliums reposent à plat sur le fond, traduisant l'affaissement du bassin. Le membre inférieur gauche est en connexion, en face antérieure: la diaphyse du fémur, son épiphyse distale, l'épiphyse proximale du tibia et sa diaphyse s'ordonnent anatomiquement. Les seuls éléments identifiables du pied sont trois métatarsiens, affaissés dans l'axe de la jambe. Du côté droit le membre inférieur a pivoté latéralement. Le fémur, toujours muni de son épiphyse distale, est en vue antéromédiale. La rotation du tibia est plus prononcée, en face médiale. Le pied a également basculé sur le côté: le tarse postérieur ainsi, peut-être, que l'os naviculaire sont en connexion, en face médiale; trois métatarsiens sont encore visibles, sur le sol, en face inférieure.

\section{MILIEU DE DÉCOMPOSITION ET TYPE DE STRUCTURE}

Le bilan taphonomique est maigre. Les seules connexions enregistrées concernent la tête et les membres inférieurs, principalement le gauche. Les ossements en jeu semblent cependant dans des situations stables, le cas de la tête méritant toutefois une mention spéciale. De l'autre côté, les dislocations avérées (et non liées à des perturbations) ne sont guère plus nombreuses. Il est délicat d'utiliser dans la discussion l'avant-bras droit. Seuls résistent le bassin et surtout les pieds où des ossements ont indiscutablement transgressé le volume corporel.

Si les dislocations qui ont affecté les pieds constituent un faible argument pour conclure à une décomposition de la totalité du cadavre dans un espace vide, la position de la tête peut constituer un argument indirect en faveur d'un aménagement. En effet, sa position redressée implique un support ou un appui. De manière plus nette, l'effet de paroi sur la gauche du sujet évoque la présence d'un élément en matière périssable, axé comme le cadavre: l'hypothèse d'un élément d'architecture (un montant) est plausible.

\section{L'INDIVIDU}

Conservation osseuse : la qualité osseuse du squelette 8 est moyenne. La fragmentation est relativement élevée, particulièrement importante au niveau du bloc craniofacial, des membres supérieurs et du squelette axial.

Âge: l'individu présente une denture mixte. Les stades de calcification des incisives et des premières molaires per- 
manentes indiquent, selon une fourchette maximale, un âge compris entre 4,5 et 8,6 ans. Nous l'intégrons dans la classe des [5-9] ans.

État buccodentaire: les dix dents déciduales conservées présentent une usure faible limitée à de petits îlots dentinaires, excepté la première molaire supérieure gauche dont les cuspides sont davantage arasées. Les huit dents permanentes actives (incisives et premières molaires) ne présentent, quant à elles, aucune usure. Aucune des dents actives (déciduales et permanentes) n'est atteinte de tartre.

Indicateurs de stress biologique: nous avons pu observer douze hypoplasies linéaires de l'émail dentaire réparties sur neuf dents et germes permanents. Les incisives et la seule canine observable présentent une à deux atteintes au niveau du tiers moyen et du tiers cervical des couronnes. De légères lignes placées sur le tiers moyen de la couronne sont visibles sur les premières molaires.

\section{LE MOBILIER}

1) Fragment proximal d'éclat dont la modalité de percussion ne peut être déterminée, en silex grenu; sur le bord gauche, un négatif postérieur aux autres de la face supérieure et formant chanfrein pourrait correspondre au reste d'un enlèvement de burin d'angle; dimensions: $29 \mathrm{~mm}$ x $26 \mathrm{~mm}$ x $7 \mathrm{~mm}$ (fig. 17, $\mathrm{n}^{\circ} 3$ ).

2) Éclat partiellement cortical par percussion indirecte (talon lisse et concave, corniche abrasée) ; le talon étant très incliné, il pourrait s'agir d'un éclat de préparation de crête telle qu'observée dans les séries régionales du Néolithique ancien.

3) Éclat partiellement cortical par percussion dure (talon lisse et épais); petites retouches marginales sur les deux bords latéraux; dimensions: $65 \mathrm{~mm}$ x $25 \mathrm{~mm}$ x $11 \mathrm{~mm}$.

4) Fragment distal d'éclat sans cortex.

Ces éléments sont issus du remplissage, aucun n'est en association avec le corps. Ils peuvent provenir d'une occupation antérieure, bouleversée par l'installation de la sépulture, comme peuvent également en témoigner des tessons de céramique appartenant à plusieurs vases présents dans le remplissage.

\section{SÉPULTURE 9}

Au décapage, la fosse de la sépulture 9 paraît de très grande taille: $3 \mathrm{~m} \times 1,40 \mathrm{~m}$. Mais $7 \mathrm{~cm}$ plus bas, elle ne mesure plus que 2,60 $\mathrm{m}$ x 1,20 $\mathrm{m}$ et adopte une forme elliptique avec un diverticule subsistant à l'angle nord (fig. 32).
Classiquement, elle est orientée nord-ouest/sud-est et son remplissage est constitué de terre fine sans gravier, riche en éléments organiques et de couleur brun foncé. La périphérie de la fosse est parsemée de petits galets et le remplissage recélait un éclat laminaire de silex, des nodules d'ocre rouge et six tessons appartenant à deux céramiques au moins.

Le diverticule contenait, outre de nombreux graviers brûlés, une huître fossile déposée au sein d'une forte concentration de charbons. Une seconde huître fut découverte $0,20 \mathrm{~m}$ sous la première. Ces fossiles proviennent des calcaires jurassiques traversés en amont, mais ne sont pas courants dans la grève charriée par l'Yonne. En trouver un fortuitement est exceptionnel; s'il est déposé dans une sépulture c'est d'autant plus rare; en trouver deux est tout à fait surprenant.

À - 0,34 m sous le décapage, les pierres ne se rencontrent qu'en bordure de la fosse. On peut supposer que la terre utilisée, relativement sèche, pouvait contenir ces pierres. Apportée sur place et versée au centre de la fosse, elle a formé un tertre sur les pentes duquel les pierres ont roulé pour venir se regrouper à sa base ou contre les parois de la cavité. À ce niveau, la fosse se réduit encore et se limite à une zone centrale de $0,90 \mathrm{~m}$ x $0,50 \mathrm{~m}$, où le squelette a été retrouvé. La fosse, de laquelle ont été extraits plus de $0,25 \mathrm{~m}^{3}$ de graviers, est largement surdimensionnée. Autour de ce surcreusement, 128 pierres ont été relevées dont 8 blocs de roche métamorphique verdâtre disposés près des épaules, des mains, des genoux et des pieds. Sous le corps subsistait une petite fosse elliptique mesurant $0,80 \mathrm{~m}$ x $0,50 \mathrm{~m}$, profonde de $5 \mathrm{~cm}$. Le fond de la sépulture est atteint à $-0,53 \mathrm{~m}$ au centre et $-0,48 \mathrm{~m}$ en périphérie.

La sépulture 9 abrite l'inhumation individuelle d'un adulte, étendu sur le dos, la tête au nord-ouest et les pieds au sud-est (fig. 33). La tête est redressée et la bouche fermée. Les épaules sont légèrement haussées. Tous les membres sont étendus: du côté droit, ils suivent strictement l'axe du corps; à gauche, le coude est très légèrement écarté du tronc et le membre inférieur converge vers le droit; les pieds sont en contact à leurs extrémités. Les mains sont lacunaires, toutefois la droite devait reposer de chant ou la paume contre le sol à côté de la hanche, la gauche dans une position sans doute symétrique. Les pieds paraissent tendus mais ils ne sont pas exempts de dislocations.

Du côté droit, le dernier métatarsien prolonge le bord latéral de la fibula. Si le fémur se trouve désaxé, on peut considérer que le membre supérieur se trouve sur la même droite. Cet effet de paroi ténu ne correspond pas à un tassement du sujet mais plutôt à un effet de délimitation linéaire. 


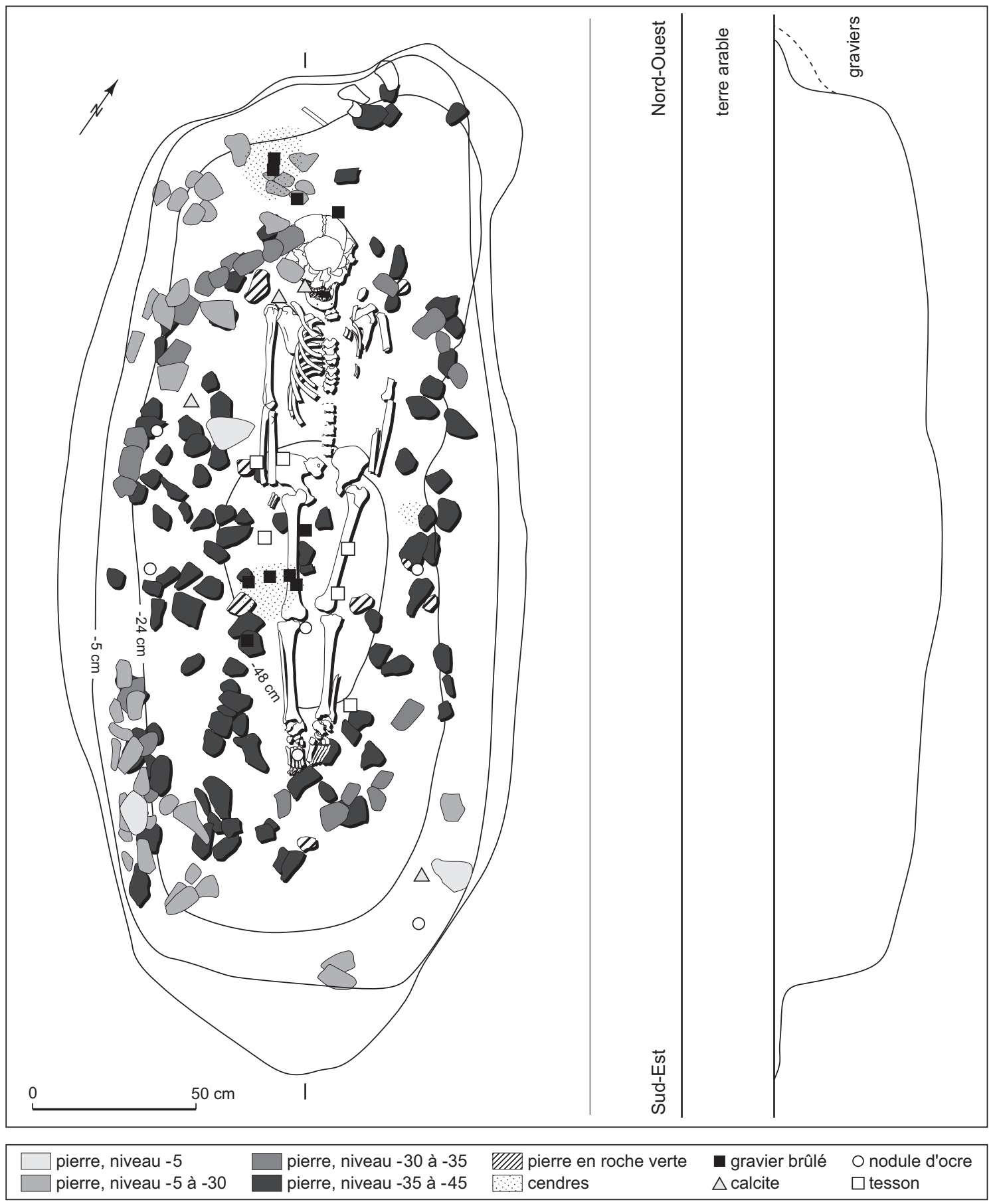

Fig. 32 - Plan et profil de la sépulture 9 (relevé: P. Chambon, CNRS).

Les photographies générales montrent de nettes différences d'altitude sur le plan transversal, pour la partie haute du corps. Les membres supérieurs dominent le tronc, la colonne vertébrale se trouvant au point le plus bas. Il s'agit là d'un effet de gouttière.

\section{CONSERVATION ET PERTURBATIONS}

Nous n'avons pu récupérer de ce sujet que la tête osseuse et l'étage cervicale de la colonne vertébrale, mais l'état général du squelette paraît bon sur les photographies. Le 


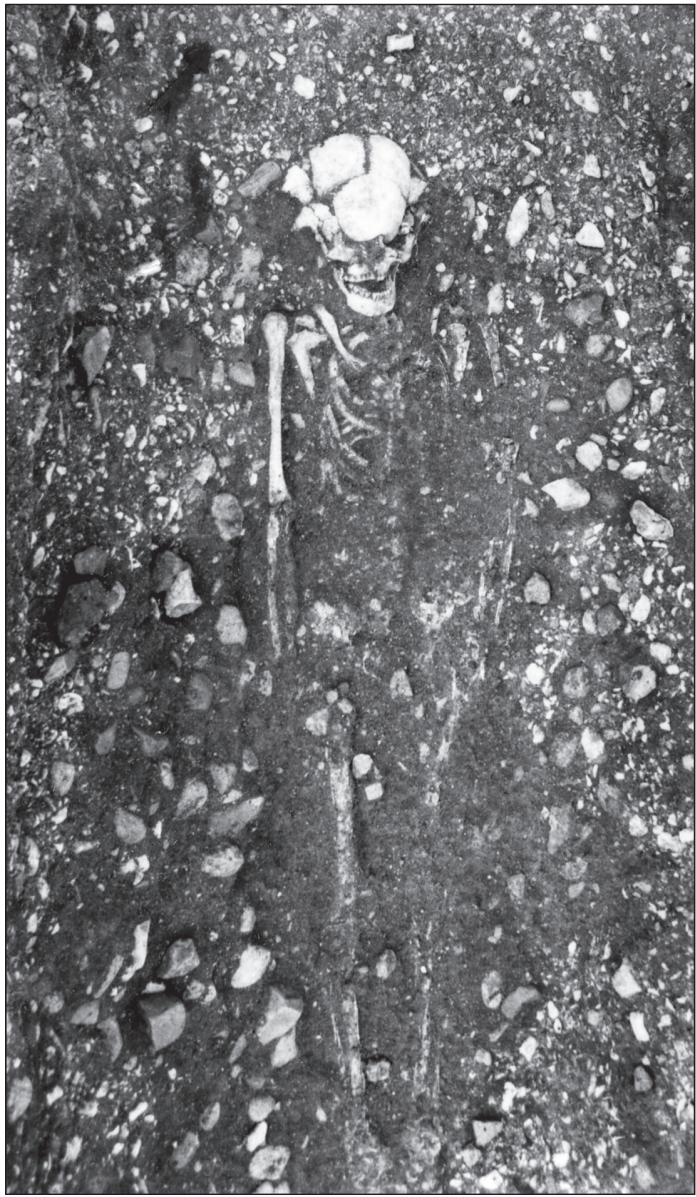

Fig. 33 - Vue générale du squelette de la sépulture 9 (cliché: J.-P. Delor).

tissu spongieux est conservé au même titre que la substance compacte de l'os: les épiphyses des os longs sont souvent présentes, comme les têtes de l'humérus ou du fémur droits; une partie des corps vertébraux est encore présente. Le plan montre toutefois d'importantes lacunes. Il manque ainsi la quasi-totalité des deux mains (nous n'avons repéré que deux métacarpiens droits), quelques vertèbres de l'étage thoracique, une grande partie de l'hémithorax gauche, ainsi que des tronçons des os du membre supérieur gauche. Pour clore cet inventaire négatif ajoutons que la moitié proximale de la fibula gauche fait également défaut.

Quel que soit le rôle joué par la conservation différentielle, elle ne peut, à elle seule, traduire un tel bilan. On peut admettre la disparition de corps vertébraux, éventuellement celle des os des mains, du fait de l'érosion physico-chimique. Cependant, l'absence du tiers mésial de l'humérus gauche, par exemple, signe le passage d'un animal à galerie. Cette intervention s'est produite alors que la sépulture était comblée, les ossements enveloppés par du sédiment: les deux extrémités de l'humérus sont demeurées à leur place initiale, comme la partie distale de la fibula. Son ou ses parcours l'ont amené principalement dans le côté gauche du sujet. Curieusement le remplissage n'a pas livré les fragments d'os manquants.

Le côté droit ne montre pas de signe du même bouleversement; l'absence de la main doit-elle être imputée à la même cause, ou bien s'agit-il d'un simple déficit de conservation? Dans ce cas, le constat peut aussi valoir pour la main gauche. Enfin, la déviation du fémur gauche ne peut pas être imputée à l'animal: la sépulture étant comblée, sa galerie n'est pas suffisamment large pour déplacer un tel os entier.

\section{CONNEXIONS ET DISLOCATIONS}

Si notre examen ne peut s'appuyer sur la majorité des articulations, des éléments diagnostiques existent.

Le bloc craniofacial et la mandibule sont en connexion. La calvaria a subi une sévère réduction de volume: le sommet de la voûte s'est affaissé dans le volume cérébral, expulsant vers l'extérieur les flancs (la partie inférieure des os pariétaux, les os temporaux). L'enchaînement des vertèbres paraît conforme à l'anatomie mais nombre d'entre elles manquent. Les côtes mises au jour traduisent l'effondrement logique du volume thoracique.

Le membre supérieur gauche ne peut pas être inclus dans cette discussion. Les liens articulaires, au sein du membre supérieur droit, sont dans l'ensemble préservés. L'extrémité acromiale de la clavicule, partiellement «verticalisée», pointe toutefois hors de l'épaule. L'humérus est dans une situation instable: il apparaît principalement par sa face latérale. Le radius s'est effondré côté médial.

Le bassin est posé à plat: les ailes des iliums se sont affaissées. La disposition respective des os coxaux et des fémurs n'implique pas de dislocation. En revanche les condyles des fémurs ne sont pas en relation étroite avec ceux des tibias. À gauche, le fémur a dévié de plus de $5 \mathrm{~cm}$ vers le plan médial, alors que du côté droit, la déviation est moindre, mais on ne peut assurer qu'elle soit à mettre au compte du fémur: le lien tibia-fibula semble conforme à l'anatomie. Les pieds présentent une bonne cohérence d'ensemble mais traduisent parfaitement une dislocation par blocs ${ }^{14}$. Ainsi, du côté

14. La dislocation par blocs correspond à l'ordre de lâchage des articulations du pied durant la décomposition. À savoir, à partir des extrémités et par «étage»: phalanges, puis métatarse, puis tarse antérieur et enfin tarse postérieur. 
droit, trois phalanges proximales gisent parallèlement, l'extrémité proximale au nord. Derrière elles viennent les cinq métatarsiens, à plat sur le sol en face supérieure, l'extrémité proximale au nord-ouest. Séparés de 1 à $2 \mathrm{~cm}$ se trouvent les os du tarse antérieur, sans réelle connexion mais avec une disposition correcte. Enfin le talus et le calcanéus sont en connexion, en face médiale (le tibia est en vue antérieure), le talon dirigé vers le nord.

\section{MILIEU DE DÉCOMPOSITION ET TYPE DE STRUCTURE}

Bien que peu nombreux, les critères suffisent à conclure à une décomposition du sujet dans un espace vide. En effet, des ossements sont sortis du volume initial du cadavre, tant au niveau de la tête qu'à celui des genoux et des pieds; il convient peut-être d'ajouter l'épaule et les fesses. Cependant, la décomposition en espace vide laisse sans explication la situation précaire de l'humérus droit, et n'explique pas, à elle seule, les ruptures des genoux.

Le déséquilibre apparent de l'humérus suppose soit un espace colmaté (contraire à l'interprétation énoncée ci-dessus), soit un appui latéral. Or l'humérus participe justement de l'effet de paroi repéré sur le côté droit du sujet. Lier effet de paroi et espace vide est une hypothèse économique. Cependant le sujet a-t-il été installé directement dans une tombe architecturée ou inséré dans un contenant rigide mobile qui a été ensuite mis en terre?

L'effet de paroi se trouve à près de $0,40 \mathrm{~m}$ du bord sud-ouest de la fosse. S'il s'agit d'une tombe architecturée, on peut s'interroger sur la présence de la paroi presque au centre de la fosse. En outre, le glissement des fémurs reste sans explication, à moins d'envisager qu'elle réside dans le fond de la structure sépulcrale. Dans ce cas, il faut que celui-ci ait été différent du fond de la fosse elle-même. Enfin, l'effet de gouttière observé sur les photographies s'accorde avec la thèse d'un contenant amovible, en dessinant même la forme interne. Le sujet a donc sans doute été inhumé dans un « cercueil $^{15}$ ».

\section{L'INDIVIDU}

Conservation osseuse: le sujet a été identifié en 1984 comme une femme adulte, relativement âgée. Si nous n'avons pas fait d'erreur d'attribution, les ossements que nous avons récupérés se limitent à la tête osseuse mais la

15. Nous nommons «cercueil», par commodité, un contenant rigide mobile, qu'il s'agisse d'un dispositif monoxyle ou d'un assemblage. voûte crânienne est incomplète, augmentée des six premières vertèbres cervicales; l'ensemble est en bon état de conservation.

Âge: la représentation osseuse du squelette, limitée à son bloc craniofacial, ne nous permet pas une estimation précise de l'âge de l'individu. Les indices que nous avons plaident en faveur d'un individu plutôt mature: suture sphéno-occipitale synostosée, troisièmes molaires calcifiées. Contrairement à l'identification faite en 1984 d'un individu âgé, aucune observation faite sur la tête osseuse ne nous permet d'aller dans ce sens: les quatre segments observables des sutures crâniennes sont ouverts et les dents présentent une usure limitée.

D'autre part, ces seuls indices ne nous autorisent pas à différencier un adolescent d'un jeune adulte. Selon divers auteurs, la suture sphéno-occipitale peut se souder entre 12 et 25 ans (Birkner, 1980; Kamina, Zwobada-Kamina, 1983; Scheuer, Black, 2000). Au vu de l'ensemble de ces données, il nous paraît plus fiable de considérer le sujet 9 comme un individu de taille adulte, c'est-à-dire d'au moins 15 ans.

État buccodentaire: à l'exception de trois dents antérieures, les arcades supérieures et inférieures sont complètes. L'usure est homogène et faible, voire nulle sur les quatre troisièmes molaires. La partie supérieure des racines est légèrement exposée indiquant une minime résorption alvéolaire. Le dépôt de tartre n'est pas systématique; de faible ampleur, il n'est présent que sur le collet des dents concernées.

Indicateurs de stress biologique: cinq hypoplasies linéaires de l'émail dentaire sont visibles sur trois incisives et deux canines. Peu prononcées, les lignes sont situées sur le tiers moyen ou sur le tiers cervical des couronnes.

\section{SÉPULTURE 10}

De taille modeste, la sépulture 10 se dessine sur le sable sous la forme d'une ellipse régulière: la fosse ne mesure que $1,25 \mathrm{~m}$ x $0,85 \mathrm{~m}$ (fig. 34). De même, son remplissage diffère des autres tombes déjà étudiées puisqu'il est composé de terre humifère mêlée de graviers et même de sable par poches. Le fond, atteint à la cote $-0,20 \mathrm{~m}$, en est arrondi en cuvette et, $0,14 \mathrm{~m}$ sous le décapage, la fosse ne mesure plus que $0,94 \mathrm{~m}$ x 0,57 m. En revanche, l'axe nordouest/sud-est est encore respecté (fig. 35).

À l'ouest, sur une surface de $0,40 \mathrm{~m}$ de diamètre et jusqu'au fond de la fosse, a été découvert ce qui a été interprété comme une incinération: os calcinés (os longs et fragments de crâne), terre brûlée, particules de charbon 


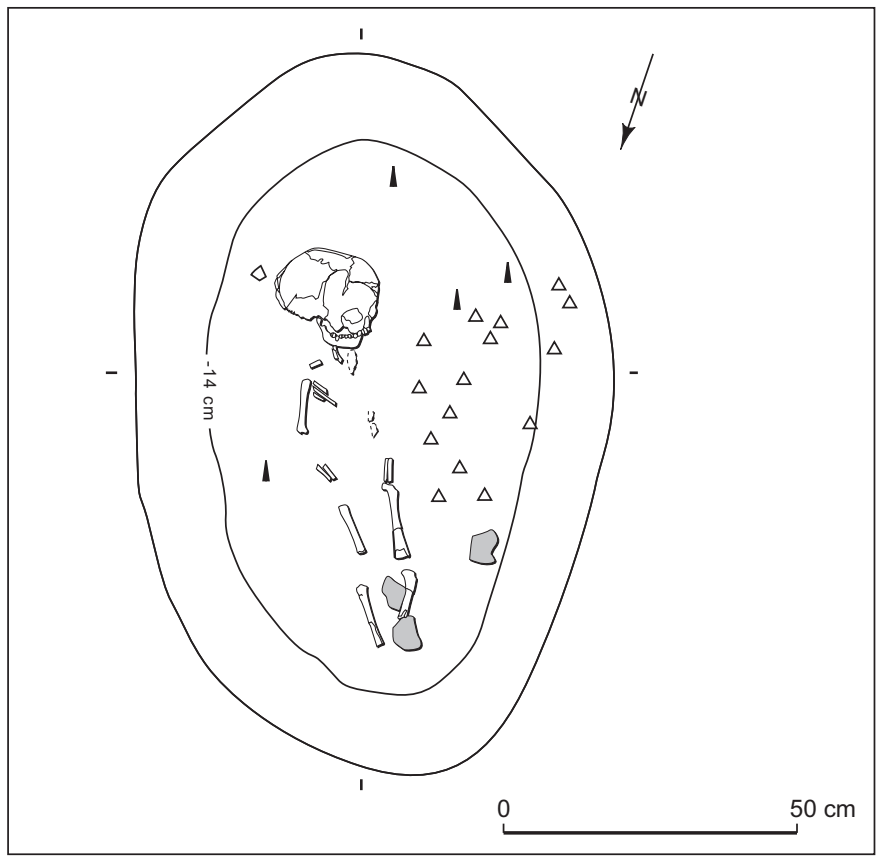

$\square$ pierre $\Delta$ os calciné $\quad$ charbon de bois

Fig. 34 - Plan de la sépulture 10 (relevé: P. Chambon, CNRS).

de bois et un éclat de silex calciné. A posteriori nous l'appelons la sépulture 10bis. De l'autre coté de la fosse, près de l'épaule droite du sujet mais quelques centimètres au-dessus du niveau de repos du squelette, furent recueillis une possible armature et un fragment d'huître fossile.

La sépulture 10 correspond à l'inhumation individuelle d'un sujet immature. Ce défunt repose en position étendue, essentiellement sur le dos, avec toutefois une composante gauche au niveau du tronc. La tête, très légèrement redressée, se trouve au sud-est, le regard vers l'ouest. La bouche est fermée. Seul le membre supérieur droit présente une flexion: le coude est décollé du côté, l'avant-bras dévie d'environ $20^{\circ}$ de l'axe tracé par le bras.

Le sujet n'occupe qu'une très faible largeur: inférieure à $0,20 \mathrm{~m}$, cette largeur maximale est atteinte par le bloc craniofacial et par la zone située entre les deux coudes (fig. 36). Cette compression du squelette s'accompagne de l'alignement des vestiges osseux sur le côté gauche: cet effet de paroi implique l'humérus, l'os «latéral ${ }^{16}$ » de l'avantbras, le fémur et le tibia.

16. Il n'est pas possible d'identifier s'il s'agit du radius ou de l'ulna.

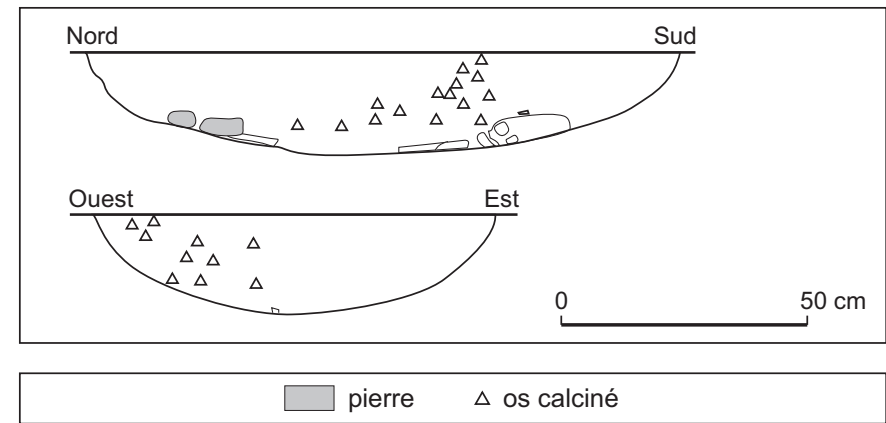

Fig. 35 - Coupes de la sépulture 10. Les os brûlés sont dispersés, mais avec un effet de pente depuis le bord ouest de la fosse. L'armature se trouve au-dessus du niveau du squelette (relevé: P. Chambon, CNRS et J.-P. Delor).

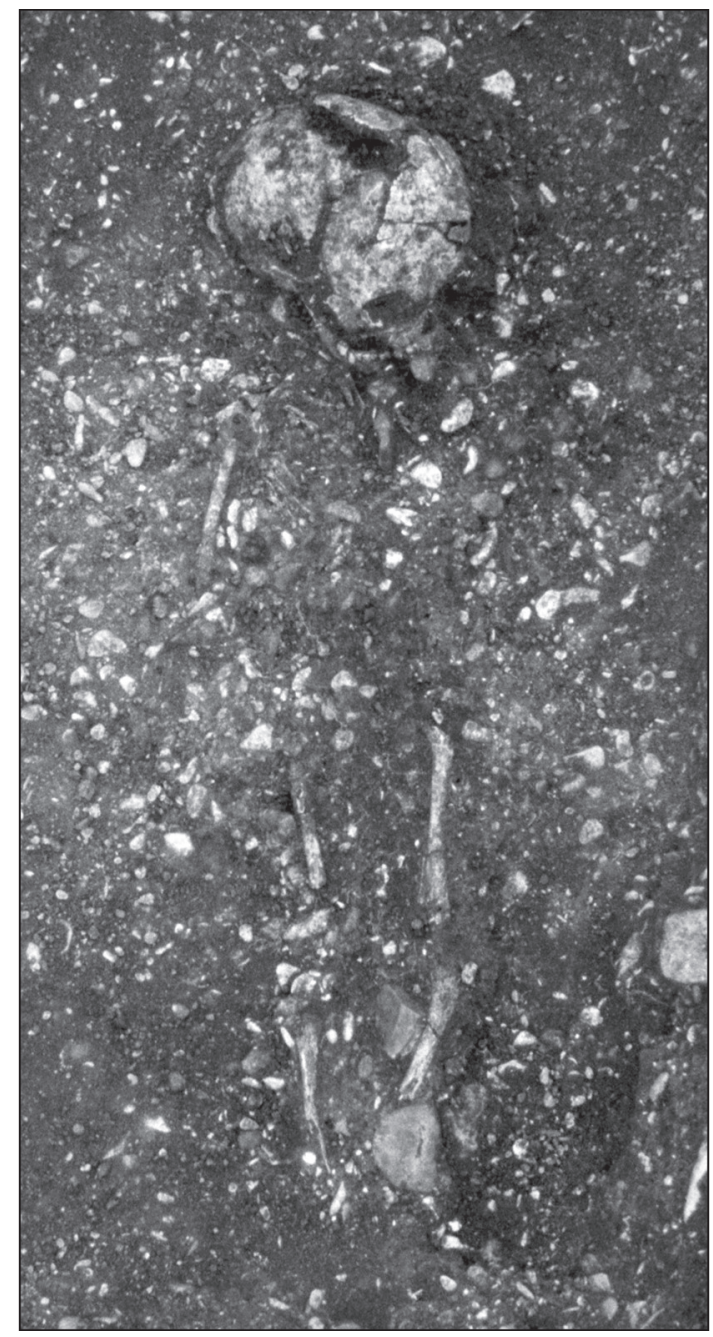

Fig. 36 - Vue générale du squelette de la sépulture 10. Le squelette n'occupe qu'une très faible largeur, moins de $20 \mathrm{~cm}$ entre les deux coudes (cliché: J.-P. Delor). 


\section{CONSERVATION ET PERTURBATIONS}

La conservation des restes osseux est mauvaise. Sur le relevé et les photographies, le squelette se résume au bloc craniofacial accompagné de la mandibule, ainsi qu'aux principaux os longs (encore n'avons-nous repéré ni l'humérus gauche ni les fibulas). Quelques fragments de côtes droites sont encore visibles dans le haut du thorax. Le reste n'est que petites esquilles, non identifiables sur les photographies.

La disposition des vestiges mis au jour ne révèle aucune incohérence anatomique et il n'y a pas lieu d'invoquer le passage d'un animal pour justifier l'absence des restes osseux. En regard de l'âge du sujet, le déficit de conservation suffit.

\section{CONNEXIONS ET DISLOCATIONS}

$\mathrm{Au}$ vu de l'inventaire, la discussion taphonomique est forcément réduite.

Le volume cérébral est partiellement préservé. Le bloc craniofacial et la mandibule, bien qu'en contact, ne sont plus en connexion : la mandibule, en face antérosupérieure, témoigne d'une rotation du bloc craniofacial durant la décomposition, sa face latérale droite étant très largement visible.

Les genoux sont disloqués. Du côté gauche, le fémur, en vue antérieure, est suivi par le tibia en face médiale: il s'ensuit un décalage au niveau du genou, correspondant au quart de tour effectué par le tibia vers l'extérieur. À droite la rupture est plus nette; la face d'apparition du fémur n'est pas identifiable, mais fémur et tibia suivent deux axes parallèles distants d'environ $5 \mathrm{~cm}$.

\section{MILIEU DE DÉCOMPOSITION ET TYPE DE STRUCTURE}

Les quelques données analysables plaident pour une décomposition en espace vide. Le bloc craniofacial a pivoté, les membres inférieurs ont été entraînés hors du volume initial du cadavre. Toutefois, dans un tel milieu, la conservation, même partielle, du volume cérébral, étonne. On peut supposer que le comblement avait commencé avant que les os de la voûte crânienne ne retrouvent leur totale indépendance.

Pour déterminer l'architecture, nous disposons de la position du sujet (incomplètement sur le dos), de l'effet de paroi et de la compression. La compression pourrait traduire un contenant étroit, toutefois elle n'est perceptible que du côté gauche du squelette: le coude droit s'écarte du tronc. Plus simplement, les trois indices peuvent avoir la même origine: le tassement du sujet contre la paroi gauche de son contenant. De tels mouvements se produisent lors du transport ou de la mise en terre du défunt dans son contenant mobile (Chambon, 1997).

\section{L'INDIVIDU}

Conservation osseuse: la qualité osseuse du squelette 10 est médiocre. Sa fragmentation importante n'a pas permis le remontage complet d'un seul os long. L'os maxillaire et la mandibule ne sont que partiellement conservés.

Âge: les arcades dentaires ne présentent aucune dent permanente. La calcification de la canine et des deux molaires déciduales indique un âge maximum entre 0,5 et 1,42 ans, soit appartenant à l'une des deux classes, [0] ou [1-4] ans. L'âge statural du sujet à partir de l'estimation de la longueur de ses fémurs (130-140 mm) corrobore ces résultats en le plaçant dans la fourchette basse des [1-4] ans. Dans un souci de fiabilité plus que de précision, nous gardons l'estimation dentaire initiale et classons l'individu 10 à cheval sur les classes des [0] et [1-4] ans.

État buccodentaire: l'usure de l'ensemble des dents observables est nulle. Aucun dépôt de tartre n'est à signaler.

\section{LE MOBILIER}

1) Possible armature tranchante trapézoïdale sur fragment mésial de lame en silex à grain très fin (les enlèvements lisibles sur la face supérieure sont parallèles et de même direction que le sens de débitage du support); comme pour certaines armatures de la sépulture 5, la retouche, bifaciale, s'est effectuée en deux temps principaux: retouche marginale sur la face supérieure, puis retouche rasante de la face inférieure; la base, particulièrement large, et le tranchant portent également quelques petites retouches marginales; dimensions: $24 \mathrm{~mm} \times 23 \mathrm{~mm} \times 4 \mathrm{~mm}$ (fig. 17, $\mathrm{n}^{\mathrm{o}} 4$ ).

2) Une esquille de silex brûlé complète l'inventaire.

Si la situation de l'armature ne permet pas de certifier son association avec l'inhumation, l'identification de la pièce invite néanmoins à la considérer comme telle. Il peut s'agir, comme précédemment, de la tête d'une flèche déposée le long du corps de cet enfant. 


\section{SÉPULTURE 10BIS}

Dans le remplissage de la sépulture 10 a été mis au jour un ensemble de restes osseux calcinés. Ils étaient dispersés sur une surface d'environ $1,20 \mathrm{~m}^{2}$ et presque toute la hauteur du remplissage (fig. 35). Il n'a pas été possible d'identifier les limites d'un quelconque dépôt. Il s'agit soit de vestiges inclus directement dans le sédiment, soit d'un dépôt remanié lors du comblement de la sépulture sousjacente.

Nous avons décompté quarante-cinq restes pour un poids total de $14,5 \mathrm{~g}$, dont certains peuvent correspondre à une fragmentation après la fouille. Dans la mesure où les vestiges déterminables sont humains, nous considérons a priori la totalité comme telle. Ils sont de couleur blanche, avec parfois des zones bleutées. Les fragments de crâne identifiés présentent des déformations, indiquant que la crémation s'est produite alors qu'il subsistait de la matière organique dans l'os. On peut présumer qu'il ne s'agit pas d'une crémation accidentelle de restes humains épars mais bien d'une véritable incinération.

Aucun reste n'est précisément localisable dans le squelette. Les vestiges appartiennent à tous les types d'ossements: os plats, os longs et os courts. Parmi les os plats nous avons repéré neuf fragments de bloc craniofacial $(6,6 \mathrm{~g})$ et huit esquilles de côtes $(0,8 \mathrm{~g})$. Les os longs sont représentés par au moins huit esquilles $(4 \mathrm{~g})$, tandis que trois fragments appartiennent à des os courts ou des extrémités d'os longs $(0,7 \mathrm{~g})$. Six pièces ont permis des recollages. Près de la moitié des restes, soit vingt, n’ont pu être déterminés; ils ne représentent toutefois que 2,4 g, soit moins de $17 \%$ du poids total.

Tous les fragments peuvent appartenir au même sujet, dont nous pouvons seulement affirmer qu'il ne s'agit pas d'un jeune sujet immature. L'épaisseur des restes, os compact ou diploé, est toutefois peu importante.

\section{SÉPULTURE 11}

La fosse contenant la sépulture 11 est en fait double. Il s'agit de deux fosses accolées et axées sensiblement nordouest/sud-est, avec un léger décalage (fig. 37). La fosse la plus petite $(1,00 \mathrm{~m}$ x $0,60 \mathrm{~m})$, placée à l'est, correspond à la tombe en elle-même alors que la grande excavation voisine $(1,40 \mathrm{~m} \times 0,80 \mathrm{~m})$ pourrait être un trou de poteau. Les deux fosses, dont le remplissage est constitué de graviers et de terre mélangés, se différencient à $9 \mathrm{~cm}$ sous le décapage et un muret de graviers les sépare alors.

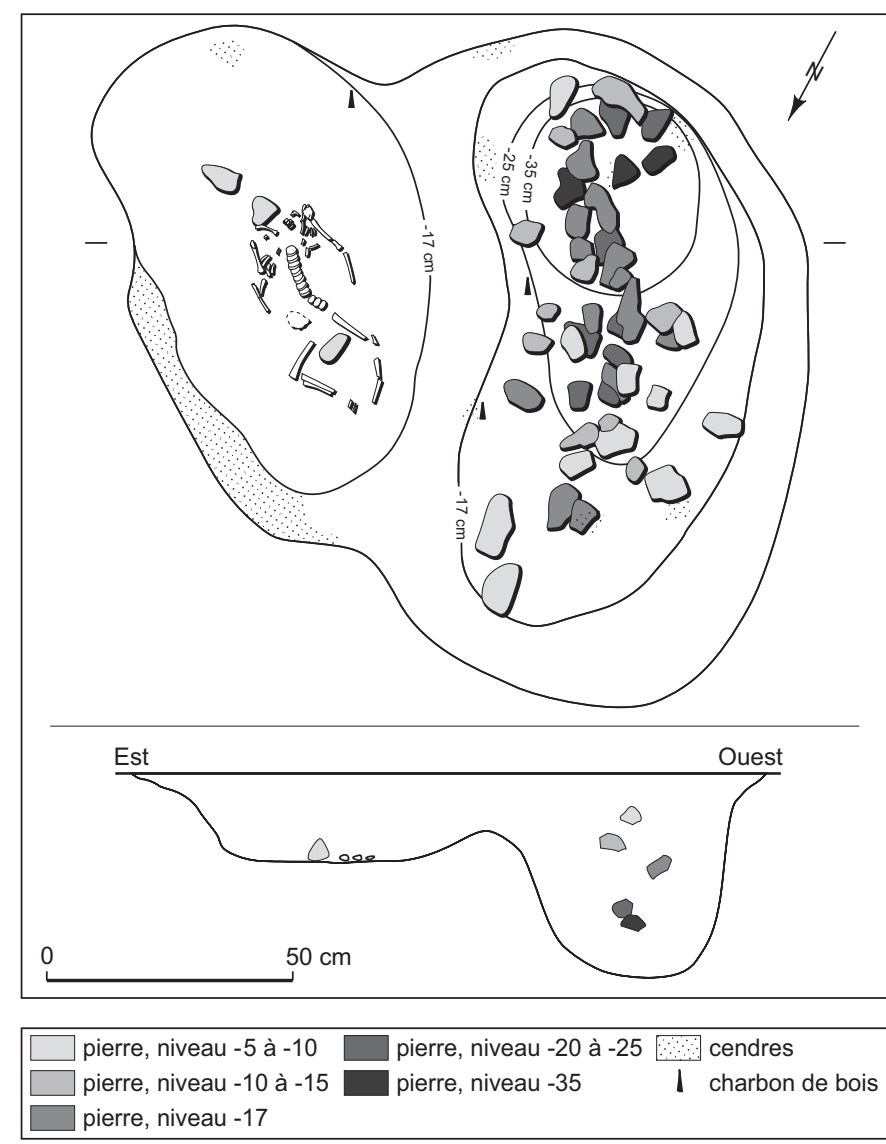

Fig. 37 - Plan et coupes de la sépulture 11 (relevé: P. Chambon, CNRS).

La tombe est peu profonde $(-0,17 \mathrm{~m})$ et une large bande charbonneuse la borde au nord-est. Le remplissage contenait un éclat de silex brûlé recueilli près du bloc craniofacial de l'inhumé.

La fosse voisine contenait quarante-six galets dont certains dépassent $0,15 \mathrm{~m}$ de plus grande longueur. Ils sont répartis dans tout le remplissage mais plus particulièrement au sud, là où la fosse est la plus profonde $(-0,42 \mathrm{~m})$. Le fond de l'excavation est presque circulaire, en «U», et mesure $0,35 \mathrm{~m}$ de diamètre. Si la paroi sud est verticale, la pente de la bordure opposée est relativement douce.

Faut-il comparer cette dernière fosse à F2 ou à F3?

Le squelette se trouvait dans le creusement le moins profond. Le sujet correspondant est immature. Il repose sur le dos selon une orientation sud-est/nord-ouest. Le membre supérieur gauche est étendu mais écarté du tronc, la position du droit, disloqué, n'est pas claire: elle peut être identique. Les hanches et les genoux sont fléchis et, à la fouille, les genoux étaient écartés d'environ $0,20 \mathrm{~m}$ 


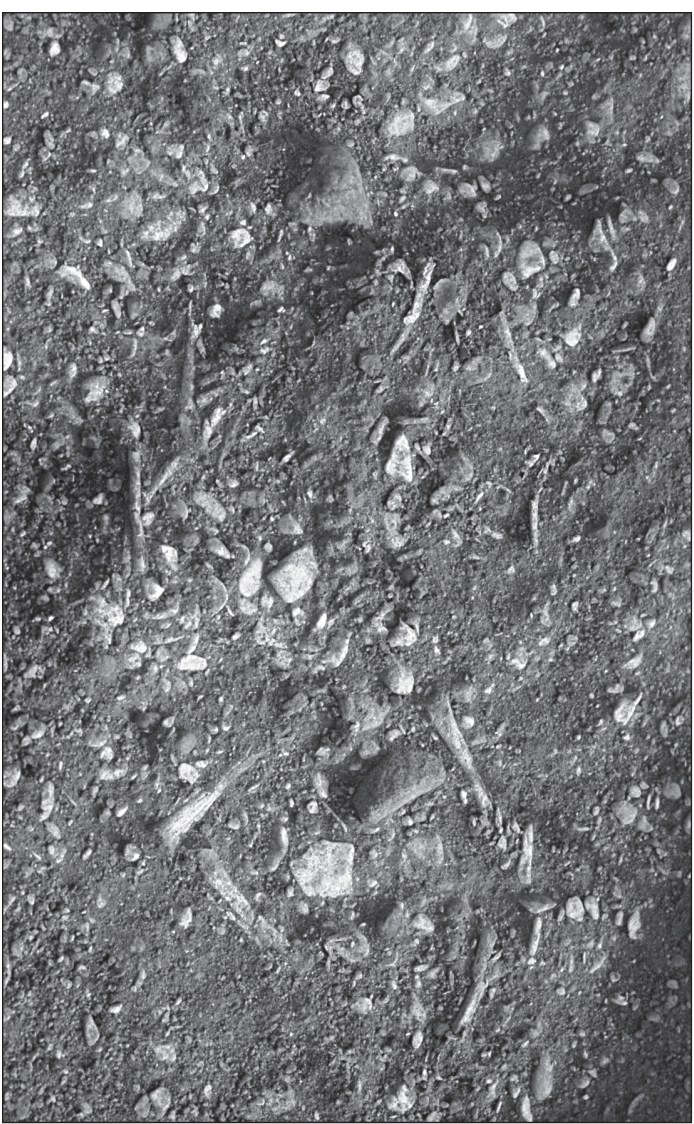

Fig. 38 - Le squelette de la sépulture 11 (cliché:J.-P. Delor).

(fig. 38); cette position correspond vraisemblablement à l'évolution du cadavre dans la structure.

\section{CONSERVATION ET PERTURBATIONS}

L'état du squelette est assez mauvais. Une trentaine de pièces seulement figurent sur le plan, à peine plus à l'inventaire, et parmi elles on déplore l'absence de l'essentiel de la tête osseuse. De manière surprenante, une part importante de la colonne vertébrale a pu être dégagée, dont une dizaine de corps vertébraux. Les diaphyses des grands os longs sont présentes, souvent incomplètes aux extrémités. Les ceintures des membres sont fragmentaires: les clavicules sont visibles sur les photographies, mais une seule scapula a été mise au jour, ainsi que la trace d'un ilium. Quelques tronçons de côtes complètent cet inventaire sommaire.

Si les restes recueillis ne comprenaient pas quelques miettes du bloc craniofacial et de la mandibule, l'absence de la tête osseuse eût justifié l'hypothèse d'un terrier. Nous aurions hésité à lui imputer le désordre régnant au sein du membre supérieur droit. En l'état, il n'y a pas de raison de considérer que les écarts par rapport au bon ordonnancement anatomique ne sont pas dus à l'évolution naturelle du cadavre dans la tombe.

\section{CONNEXIONS ET DISLOCATIONS}

La discussion taphonomique est grevée par la piètre conservation des restes osseux.

Pour le squelette axial, trois segments de colonne vertébrale ont été mis au jour. Le segment supérieur est nettement disjoint du suivant: le hiatus correspond certes à la disparition de vertèbres, toutefois leur restitution suppose une torsion considérable pour joindre les deux segments. Les fragments de côtes occupent une largeur importante, mais il n'est pas possible d'affirmer qu'ils outrepassent le volume initial du thorax.

Les membres supérieurs recèlent les dislocations les plus nettes. Du côté gauche, la position de la scapula est cohérente avec le segment supérieur de la colonne vertébrale; celle de l'humérus s'accommode assez du second segment de la colonne vertébrale. Au final, leur disposition réciproque n'est pas conforme à l'anatomie: la partie proximale de l'humérus est décalée de la cavité glénoïdale de près de $4 \mathrm{~cm}$, en direction de la colonne. En revanche, la situation de l'os de l'avant-bras n'implique pas une dislocation du coude. À droite, le membre supérieur est totalement déconnecté: la clavicule paraît bien haute. L'extrémité proximale du radius est écartée latéralement de près de $5 \mathrm{~cm}$ de la partie distale de l'humérus. L'ulna croise le radius à mi-diaphyse.

La situation des membres inférieurs n'est guère différente. Le fémur gauche se situe sur le même axe que le segment inférieur de la colonne vertébrale, impliquant une dislocation du bassin ou de la hanche. Du côté droit, l'extrémité proximale des os de la jambe se trouve en regard de la partie distale de la diaphyse du fémur, non de l'extrémité elle-même.

\section{MILIEU DE DÉCOMPOSITION ET TYPE DE STRUCTURE}

Les dislocations sont assez manifestes et justifient le verdict d'une décomposition en espace vide. En conséquence, il est nécessaire de revenir sur la position du sujet. La position des membres inférieurs peut très bien résulter de la décomposition. Les genoux étaient fléchis - mais ils pouvaient être surélevés - et joints. 
Aucun effet de paroi ou de compression ne vient éclairer le type d'architecture. La structure qui abritait le cadavre était toutefois assez large (par comparaison avec la sépulture 10, par exemple) pour permettre des déplacements d'ossements de grande amplitude. Enfin, si la présence d'un vide durant la décomposition est une condition nécessaire aux dislocations décrites, elle paraît toutefois insuffisante à les justifier. Comment expliquer le décalage entre l'ensemble constitué par le segment supérieur de la colonne et la scapula gauche, d'une part, et le segment suivant de la colonne vertébrale et la partie libre du membre supérieur gauche, d'autre part? De la même façon, le déplacement du radius droit ou les anomalies dans la zone du bassin impliquent une condition supplémentaire. La désagrégation d'un élément sous-jacent est l'hypothèse que nous retiendrons. Là encore, cet élément en matière périssable sous le cadavre suggère que la structure qui a accueilli ce dernier ait eu un fond; elle remplit donc les conditions d'un contenant amovible.

\section{L'INDIVIDU}

Conservation osseuse: la conservation du squelette 11 est mauvaise, tant en termes de représentation que de qualité osseuse. Le bloc craniofacial n'est représenté que par les pétreux, la partie latérale droite de l'occipital, un fragment du sphénoïde et douze germes dentaires isolés; les os longs des membres sont incomplets.

Âge: les douze germes dentaires inventoriés ne sont que déciduaux. Les stades de calcification des molaires correspondent à un enfant décédé avant l'âge de 1 an (0,08-0,5 ans). Une estimation relativement précise de la longueur des fémurs (environ $95 \mathrm{~mm}$ ) a pu être faite à l'aide des restes des deux fémurs (respectivement représentés par leur deux tiers proximal et distal). D’après les régressions de P. Sellier ${ }^{17}$ et les tables de références de G. Olivier et H. Pineau (1958), cet enfant aurait un âge entre 3,65 et 4,83 mois. Nous classons donc l'individu 11 dans la classe [0] an.

\section{SÉPULTURE 12}

De petite taille et destinée à un enfant (la fosse mesure $1,10 \mathrm{~m} \times 0,70 \mathrm{~m}$ ), la sépulture 12 est orientée nord-ouest/ sud-est avec un angle de $50^{\circ}$ par rapport au nord (fig. 39).

17. «L'estimation de l'âge des foetus et des enfants morts en période périnatale: une révision de Fazekas et Kosa (1978) ", communication non publiée de P. Sellier lors du XXI colloque du Groupement des anthropologistes de langue française, Bordeaux, 13-15 mai 1993.

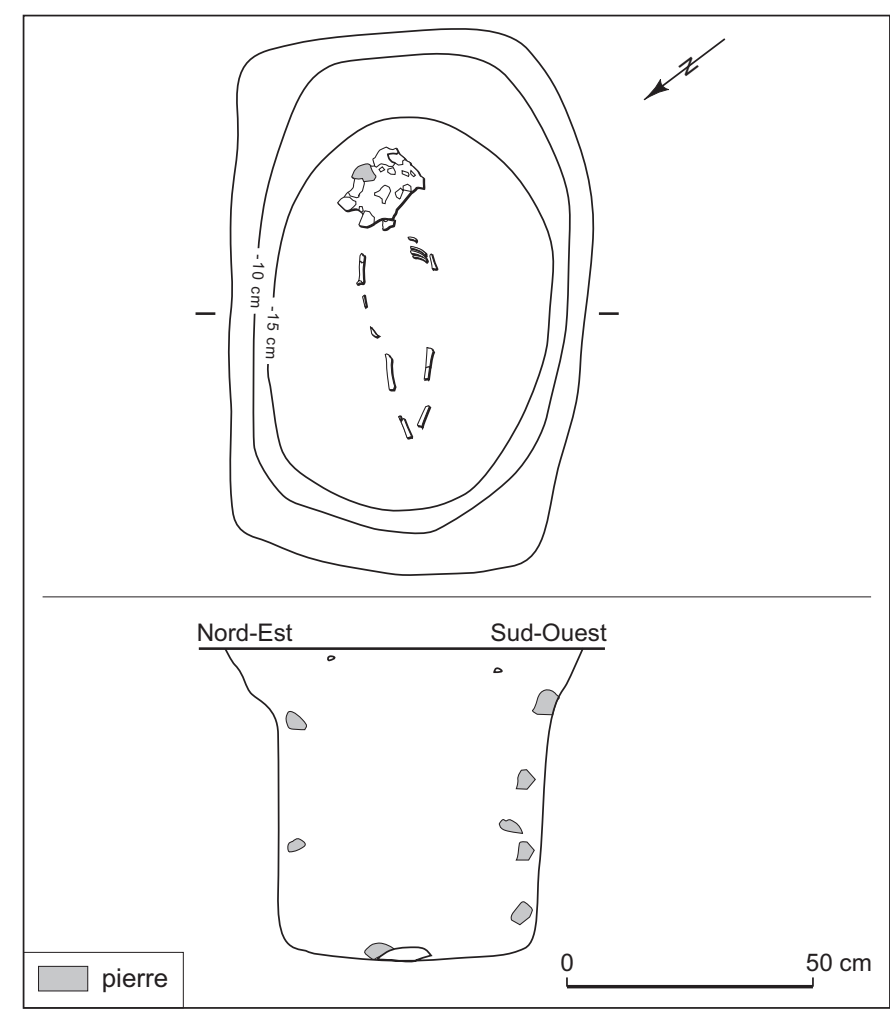

Fig. 39 - Plan et coupes de la sépulture 12. La position des pierres contre la paroi de la fosse respecte une limite verticale virtuelle, à près de $0,10 \mathrm{~m}$ du bord de la fosse (relevé: P. Chambon, CNRS et J.-P. Delor).

Son remplissage est constitué de terre arable, fine et sans gravier, qu'on ne trouve pas sur le site. Toutefois, 51 galets de 3-10 cm de diamètre se sont positionnés sur le pourtour de la fosse lors de son remplissage. Trois petits outils de silex (coches et burin ${ }^{18}$ ), des nodules d'ocre et un fragment d'huître fossile ont aussi été recueillis. À partir du niveau - 0,15 m jusqu'à l'approche du fond, à - 0,62 m, les parois de la sépulture sont verticales. Le fond, qui mesure $0,80 \mathrm{~m} \times 0,55 \mathrm{~m}$, est plat.

La sépulture 12 correspond à l'inhumation individuelle d'un sujet immature. Il repose en position étendue sur le dos, la tête au sud-est et les pieds au sud-ouest. Le membre supérieur droit, comme les membres inférieurs, sont étendus dans l'axe du corps.

Le plan et les photographies permettent de discerner une dizaine de restes seulement, identifiés d'après leur situation. Hormis un fragment de calvaria, on distingue l'humérus droit et sans doute une portion de l'humérus gauche, un os de l'avant-bras droit, les fémurs et les tibias,

18. Le burin et l'une des coches n'ont pas été retrouvés. 
ainsi que quatre traces parallèles de côtes gauches. La disposition de ces quelques vestiges, sans satisfaire totalement aux exigences anatomiques, n'implique aucun bouleversement extérieur.

L'analyse des liens anatomiques n'est pas réalisable au vu de l'état du squelette. On peut seulement noter que les traces correspondant au fémur et au tibia gauches indiquent une disjonction du genou. Par ailleurs, les parties proximales des fémurs paraissent trop écartées en regard de la taille du défunt. Il ne s'agit toutefois que de traces.

Nous ne soutiendrons aucune hypothèse quant au milieu de décomposition. Des présomptions de dislocation plaideraient pour un espace vide mais il ne s'agit que de présomptions. Le meilleur indice d'une architecture sépulcrale n'est, cette fois-ci, pas l'analyse taphonomique mais la coupe transversale de la fosse. La position des pierres contre la paroi de la fosse respecte une limite virtuelle, un effet de paroi : cet alignement des pierres dans le remplissage est indiscutable du côté sud-ouest.

La conservation du squelette 12 est très mauvaise. La tête osseuse n'est représentée que par les pétreux fragmentaires; aucune épiphyse n'est conservée; le squelette axial et les ceintures des membres supérieurs et inférieurs font défaut.

Le format des fragments osseux conservés nous indique qu'il s'agit d'un très jeune sujet. Néanmoins, en l'absence de reste dentaire ou de longueur diaphysaire, il est difficile d'estimer un âge précis. Notre seul recours a été une comparaison visuelle avec les autres sujets immatures de la nécropole dont l'âge avait pu être estimé avec fiabilité. Le format des fémurs de ce sujet s'apparente nettement à celui de l'individu 10, quoique légèrement inférieur, mais reste plus important que celui des fémurs de l'individu 11. Les classes d'âge de [0]/[1-4] et [0] ans ayant été respectivement attribuées à ces deux individus, il ne semble pas risqué de donner un âge à l'individu 12 à cheval entre les [0] et [1-4] ans ${ }^{19}$.

Le mobilier que nous avons retrouvé comprend une coche sur lame courte par percussion indirecte en silex à grain très fin (talon lisse et concave, corniche légèrement esquillée) (fig. 17, $\mathrm{n}^{\circ}$ 5), ainsi qu'une lame à trois pans, montrant un sens de débitage unidirectionnel; dimensions : $33 \mathrm{~mm}$ x $16 \mathrm{~mm}$ x $4 \mathrm{~mm}$. Cet élément, provenant apparemment du remplissage, ressemble à certaines productions

19. Parmi les restes du sujet 12 figure un fragment d'os brûlé. S'il s'agit d'un reste humain, il appartient à un métatarsien d'un adulte ou d'un adolescent. Nous ne pouvons pas assurer qu'il ne s'agit pas d'un mélange.
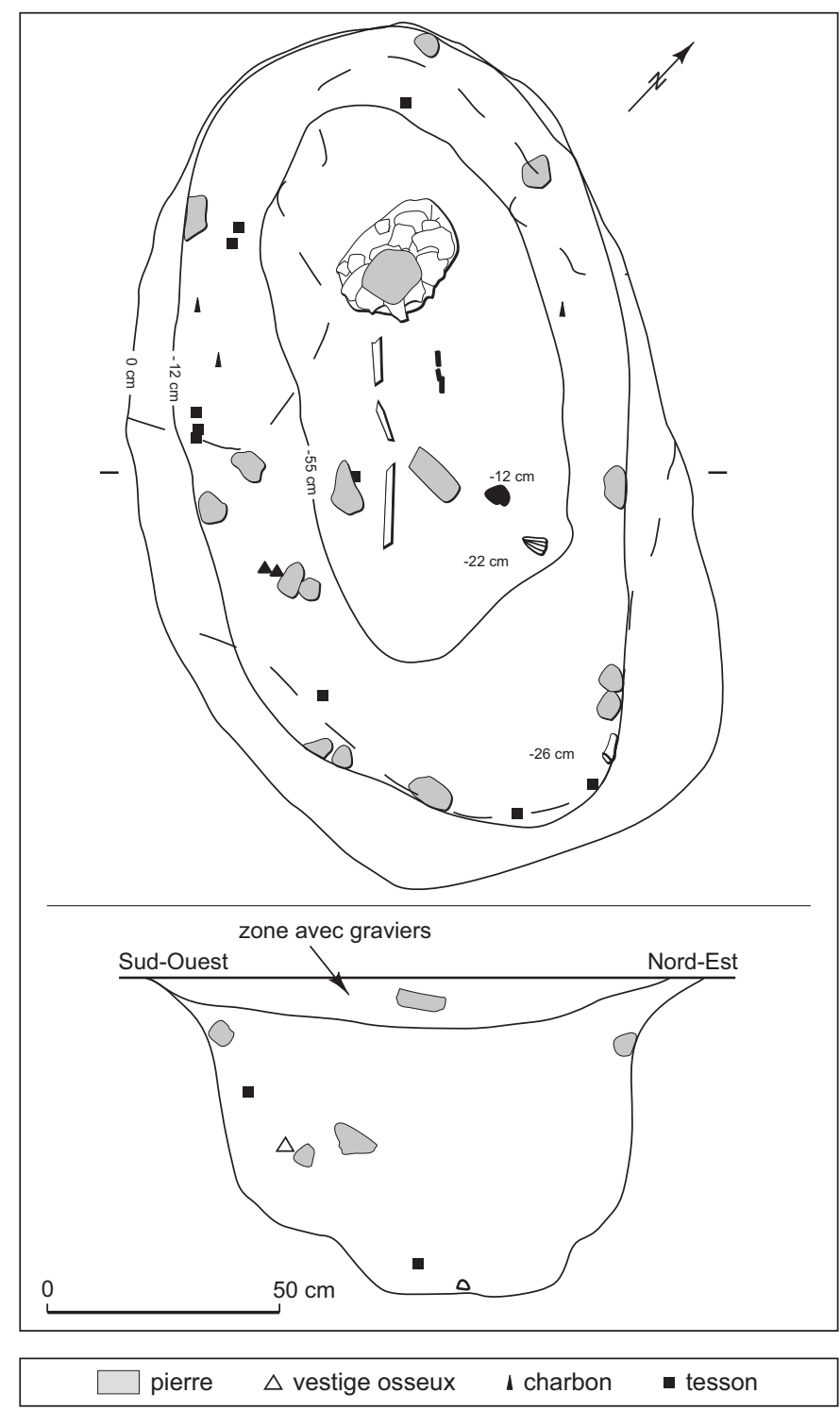

Fig. 40 - Plan et coupes de la sépulture 13 (relevé: P. Chambon, CNRS et J.-P. Delor).

lithiques du Néolithique ancien régional. Il pourrait donc être intrusif.

\section{SÉPULTURE 13}

La sépulture 13 est orientée nord-ouest/sud-est et la forme de la fosse est elliptique. Elle mesure 1,70 m x 1,00 m et son remplissage est en surface fortement chargé en graviers (fig. 40). Forme, orientation, dimensions et remplissage se modifient ensuite: l'extrémité sud se déporte légèrement vers l'est, le remplissage devient vite foncé et 


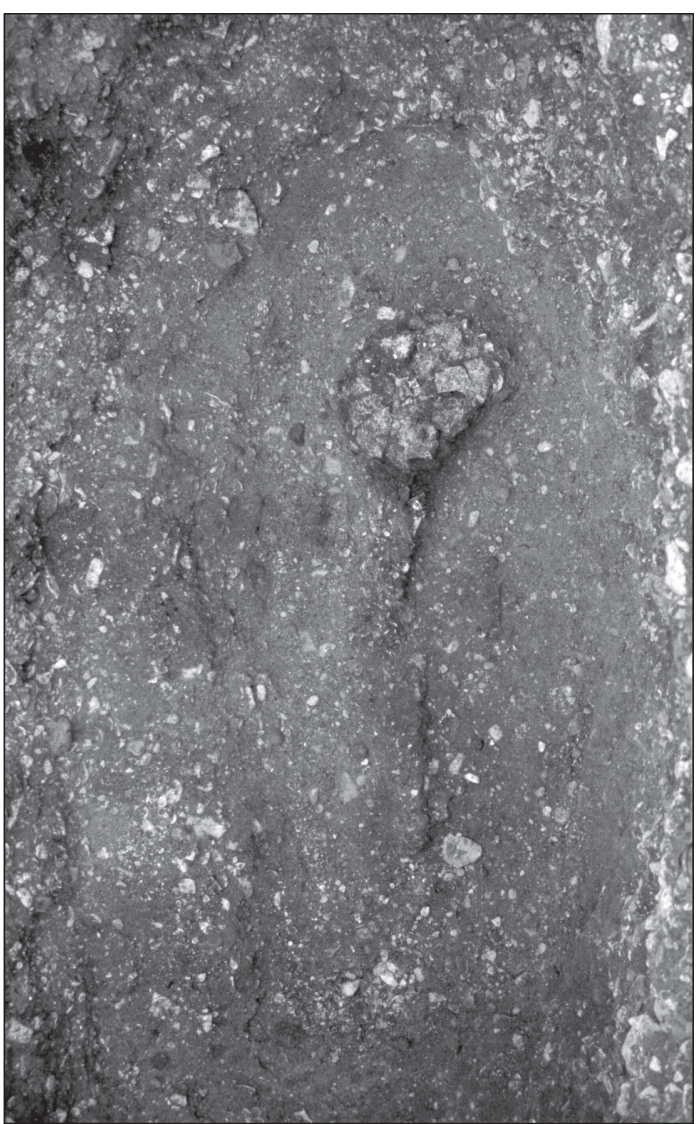

Fig. 41 - Vue des restes osseux de la sépulture 13 (cliché: J.-P. Delor).

constitué presque uniquement de terre humifère, notamment aux extrémités, confirmant un tassement central. Au niveau - 0,40 m, la zone sud-ouest présente un aspect rubéfié comme si un feu avait été allumé dans la fosse. Les dimensions de celle-ci se réduisent soudain au niveau - 0,55 m $(1,20 \mathrm{~m} \times 0,55 \mathrm{~m})$ et c'est dans ce surcreusement à fond plat et de $0,10 \mathrm{~m}$ de profondeur que le squelette a été découvert.

La tombe 13 est celle qui a livré les restes humains en plus mauvais état. Elle comprenait une inhumation individuelle. Le sujet immature reposait sans doute sur le dos, selon une orientation nord-ouest/sud-est.

Le squelette se résume au bloc craniofacial et à quatre fragments d'os longs, sans doute les deux humérus, un os de l'avant-bras droit et le fémur droit (fig. 41).

Le bloc craniofacial est écrasé, le volume cérébral réduit; il apparaît sans doute en partie par sa face supérieure mais il n'est pas possible de préciser davantage.

Il va de soi que les liaisons articulaires ne sont pas observables.
Aucun reste osseux appartenant au sujet 13 ne nous est parvenu. Les relevés de terrain montrent une distance maximale de $1 \mathrm{~m}$ entre le bord du bloc craniofacial et le bord opposé du fond de fosse. Même en suggérant que les membres inférieurs du sujet étaient fléchis, on peut difficilement lui restituer une stature supérieure à $1,05 \mathrm{~m}$, ce qui paraît déjà extrême. D’après les tables de M. Sempé (1979), un enfant (fille ou garçon) de cette stature peut être âgé de 3,5-5,5 ans. Bien que peu précis, il nous paraît plus prudent de classer ce sujet dans l'une des deux classes: [1-4] ou [5-9] ans.

Huit tessons appartenant à deux poteries et du mobilier lithique ont été découverts dans le remplissage; il s'agit d'un éclat, d'un nucléus à lamelles et d'un outil cassé (fragment de pic) en silex.

Il est signalé trois pièces lithiques provenant du remplissage (un nucléus, un fragment de pic et un éclat) que nous n'avons pas pu observer.

\section{LES PRATIQUES FUNÉRAIRES, TRAIT PAR TRAIT}

\section{LES FOSSES}

Les dimensions et les morphologies des fosses sépulcrales présentent une large diversité, depuis le creusement en baignoire très régulier de la sépulture 12, avec des parois verticales sur plus de $0,50 \mathrm{~m}$ de haut, jusqu'à la curieuse fosse double de la sépulture 11 où une seconde excavation au plan réniforme, plus profonde, jouxte celle qui abrite le sujet. Au niveau du décapage, hormis ladite tombe 12, les contours dessinent des formes subrectangulaires ou plus ou moins ovales. Les plans les plus réguliers sont proposés par les sépultures 2, 3, 7, 9 (hors diverticule nord) et 12. La profondeur oscille entre quelques centimètres sous le décapage, dans le cas de la sépulture 1 (0,10 m pour la sépulture 6) à $0,65 \mathrm{~m}$ pour la plus profonde, la sépulture 13 , soit près de $1 \mathrm{~m}$ sous le niveau actuel du sol. Assez logiquement, une profondeur importante implique des parois verticales. Les fosses les plus régulières figurent donc parmi les profondes: les tombes 7, 9 et 12 en sont les meilleurs exemples.

Les proportions des fosses excèdent celles que nécessite la seule inscription d'un cadavre en position étendue. Hormis la sépulture 7, dont la longueur ne dépasse pas $1,85 \mathrm{~m}$, les fosses abritant des adultes dépassent $2 \mathrm{~m}$ de longueur, et atteignent jusqu'à $3 \mathrm{~m}$ pour la sépulture 9. Les largeurs sont à l'avenant, avec souvent plus de $1 \mathrm{~m}$. D’une manière générale, le rapport longueur/largeur s'établit 


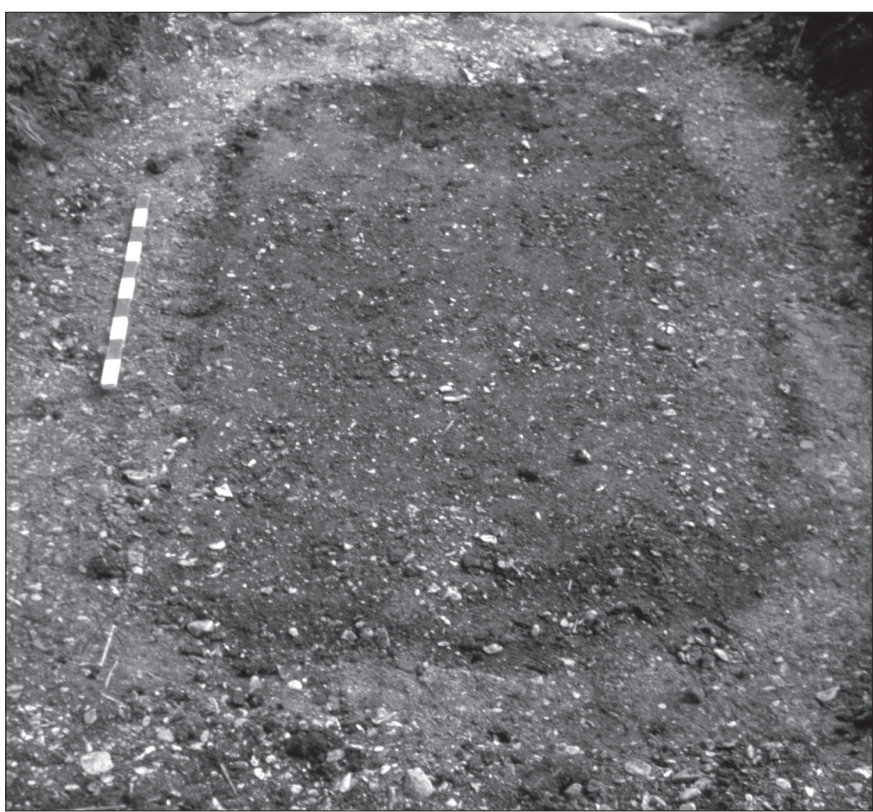

Fig. 42 - La sépulture 3 sous la terre arable. Le remplissage homogène ne contient qu'une faible quantité de graviers (cliché: J.-L. Tainturier).

autour de 2. La disproportion des fosses sépulcrales comportant des sujets immatures est du même ordre, toutefois, la largeur n'est pas aussi modulable que la longueur: le rapport longueur/largeur oscille autour de 1,6.

Les remplissages sont homogènes d'un point de vue sédimentaire: il s'agit d'un limon organique brun foncé contenant une part faible de graviers (fig. 42). Dans aucun cas une distinction verticale ou horizontale n'a été remarquée. La faible quantité de graviers dans les remplissages est frappante, notamment pour les sépultures les plus profondes. C'est en effet ce matériau qui a été majoritairement extrait lors du creusement. Un tel paradoxe plaide pour la disjonction du creusement et du comblement, ce qui s'accorde avec l'espace vide durant la décomposition des corps, mais ceci n'est pas suffisant. Si ce sédiment avait été remis par-dessus la tombe, il aurait finalement dû la remplir, ce qui n'est pas le cas.

Les galets constituent d'autres éléments remarquables des remplissages. Leur inégale densité suggère qu'ils ont parfois été délibérément inclus dans la sépulture: le remplissage de la tombe 9 en a ainsi livré près d'une centaine, alors que trois seulement figurent sur les relevés de la tombe 10. Leur disposition appelle différentes remarques. Tout d'abord, ils se rencontrent majoritairement sur le pourtour de la fosse, surtout dans les niveaux supérieurs: c'est le cas pour les sépultures 5, 9, 12 et 13; la coupe de la sépulture 12 montre clairement que les galets respectent une limite verticale de 0,10 m à l'intérieur de la paroi de la fosse. Ensuite, les galets situés à la base du remplissage sont disjoints du sujet: dans la sépulture 5, on les retrouve à la droite du sujet, au-delà de la limite marquée par l'alignement des vestiges osseux; cette situation est particulièrement nette dans la sépulture 9, pour laquelle la projection de toutes les pierres sur le plan ne masque aucun vestige osseux. Enfin, dans les tombes 6 et 9, l'agencement d'une série de galets définit une limite en arc de cercle, sur laquelle ils viennent s'appuyer: pour la tombe 6 , cet arc n'est perceptible qu'audessus de la tête, alors qu'au sein de la tombe 9 on suit cette limite depuis le nord-ouest jusqu'au sud.

\section{LE MODE D'INHUMATION ET LE DISPOSITIF SÉPULCRAL}

Le sujet de la sépulture 1 repose en position fléchie, les membres inférieurs vers la gauche, le buste largement tourné contre le sol. À l'exception de cette sépulture, tous les sujets inhumés gisent en position étendue sur le dos. Le très jeune enfant de la tombe 11 devait avoir, à l'origine, les genoux surélevés et joints. Lorsqu'on peut l'observer, la tête était redressée lors de l'inhumation: seule la tombe 7 échappe à cette règle, ainsi que, peut-être, la tombe 6. Dans quelle mesure peut-on toutefois assurer qu'un basculement secondaire de la tête n'a pas effacé toutes les traces de la position d'origine?

Les squelettes sont situés au centre de la fosse; seul le sujet 7 est déporté vers la paroi est de la fosse (à moins de $0,20 \mathrm{~m}$ de celle-ci), et dans une moindre mesure le sujet de la tombe 3 , mais il reste à bonne distance de la paroi de la fosse. Hormis ladite tombe 7, les ossements ne jouxtent jamais les parois de la fosse.

Les cadavres se sont décomposés à l'abri du sédiment environnant. La dislocation récurrente d'articulations s'accompagne dans neuf cas d'effets de paroi latéraux ou bilatéraux (sépultures 2 et 6). Ces effets traduisent soit l'inscription du sujet dans un contenant étriqué (effet de paroi bilatéral), soit le tassement du sujet contre un des montants de la structure qui l'accueille; la cause d'un tel tassement est le fort pendage transversal du fond de la sépulture ou, plus généralement, les inévitables cahots occasionnés par le transport du contenant rigide (avec le défunt à l'intérieur) et sa descente dans la fosse. 
S'il semble logique de lier la décomposition en espace vide avec l'existence des effets de paroi, et donc de conclure à l'inscription du sujet dans un contenant rigide mobile clos, une telle hypothèse se heurte, dans deux cas au moins, à une difficulté. En effet, tant dans la tombe 2 que dans la tombe 6 , les effets de parois bilatéraux ne concernent que la moitié inférieure du squelette, tandis que les membres supérieurs les transgressent. Si on conserve le lien effet de paroi-structure sépulcrale, un tel constat offre une alternative: les limites latérales fluctuantes du contenant indiquent son évasement depuis le fond jusqu'à l'ouverture (mais nous manquons de profils transversaux pour le squelette ou d'altitudes pour les ossements); le contenant n'est pas clos et les membres supérieurs chevauchent ses rebords. Cette seconde hypothèse imposerait l'existence d'un dispositif supplémentaire pour garantir l'espace vide durant la décomposition: du simple couvercle sur la fosse à l'implantation d'une véritable architecture dans la fosse.

La thèse d'une double structure trouve un soutien ténu par les observations réalisées sur les galets. Les galets les plus profonds respectent l'espace du cadavre (sépultures 3, 5 et 9): ils ne semblent pas jouer un rôle de calage du cercueil (sauf peut-être les pierres en roche verte de la tombe 9), mais ont sans doute buté contre sa paroi. La distribution des galets sur le pourtour des fosses, voire les arcs de cercles qu'ils dessinent ne peuvent correspondre à ces contenants initiaux, car les limites ne coïncident pas. Cependant, ils semblent bien prendre appui sur des éléments aujourd'hui disparus. On peut envisager qu'il s'agit d'un coffrage aménagé au sein même de la fosse.

Conclure sur les architectures sépulcrales serait bien imprudent. Les tombes partagent bien des caractéristiques, mais leurs particularités interdisent de considérer a priori qu'elles répondent d'un modèle unique. La sépulture 1, différente à tous points de vue, en est l'illustration et cette opposition première ne peut conduire à passer sous silence des différences dans les douze autres sépultures. En outre, superposer des traits observés dans chacune des sépultures conduit inévitablement à façonner une chimère. Les faits les plus tangibles suggèrent que les défunts ont été transportés dans des contenants rigides mobiles et installés ainsi dans les tombes. Dans plusieurs de ces fosses une structure de type coffrage (un caveau) avait préalablement été construite.

\section{LES INHUMÉS}

\section{CHOIX DES MÉTHODES ANTHROPOLOGIQUES ET DONNÉES BRUTES}

\section{Estimation de l'âge des sujets immatures}

Notre objectif a été de classer l'ensemble des individus immatures en classes d'âge quinquennales, conformément aux tables de S. Ledermann (1969). Nous avons privilégié l'estimation de l'âge au décès des sujets immatures à partir de leur maturation dentaire. Notre choix s'est tourné vers la méthode de C. F. A. Moorrees, E. A. Fanning et E. E. Hunt Jr. (1963a et b) pour les avantages qu'elle présente, à la fois théoriques (résultats inclus dans un intervalle de confiance à $95 \%$ ) et pratiques: applicable sur dents et germes isolés, cette méthode nous a permis de pallier le problème de conservation osseuse (exemple de l'individu 11).

En l'absence de reste dentaire, l'âge osseux, quoique moins performant, reste le seul disponible. Deux facteurs sont concernés: la croissance et la maturation. Le premier critère (taille des os longs) nous a permis de préciser l'âge des très jeunes enfants $(10,11,12)$ et de distinguer les individus décédés en période périnatale de ceux ayant survécu quelques mois, voire au moins un an (Olivier, Pineau, 1958 et Sellier, comm. pers. ${ }^{20}$ ). L'estimation de la stature à partir des relevés de terrain des sujets 5 et 13, pour lesquels nous n'avions pas les ossements, a été comparée aux tables de M. Sempé et G. Pedron (1979). L'ultime étape de la maturation osseuse, soit la fusion des centres d'ossification secondaires aux centres primaires, a été l'indice le plus pertinent à la reconnaissance de l'adolescent 7. En l'absence de longueur diaphysaire (individus 12 et 3bis), c'est grâce à un classement des individus âgés et à leur comparaison visuelle que nous avons intégré ces sujets en différentes classes d'âge. Le classement final de chaque individu immature de la nécropole de Chichery est présenté dans le tableau I en fonction des méthodes d'estimation d'âge appliquées.

\section{Estimation de l'âge des adultes}

L'observation de la maturation complète des derniers points d'ossification du squelette (extrémité sternale de la clavicule et crête iliaque) nous a permis de distinguer les sujets de plus de 25 ans (Owings Webb, Suchey Myers, 1985): sépultures $1,2,3,4$ et 6 . Dans la mesure où l'état de conservation des squelettes le permettait, nous avons

20. Voir note 17, p. 157. 


\begin{tabular}{|c|c|c|c|c|c|}
\hline Individu & $\begin{array}{c}\text { Âge } \\
\text { dentaire }\end{array}$ & $\begin{array}{c}\text { Âge } \\
\text { diaphysaire }\end{array}$ & $\begin{array}{c}\text { Âge } \\
\text { maturation }\end{array}$ & Autre* $^{*}$ & $\begin{array}{c}\text { Estimation } \\
\text { finale }\end{array}$ \\
\hline 3bis & & & & & {$[\mathrm{P}]$} \\
\hline $\mathbf{1 1}$ & $0,08-0,5$ ans & $3,7-4,8$ mois & & & {$[0]$} \\
\hline $\mathbf{1 2}$ & & & & & {$[0][1-4]$} \\
\hline $\mathbf{1 0}$ & $0,5-1,42$ ans & $17,2-19,6$ mois & & & {$[0][1-4]$} \\
\hline $\mathbf{1 3}$ & & & & $3,5-5,5$ ans & {$[1-4][5-9]$} \\
\hline $\mathbf{5}$ & & & & $3,5-5,5$ ans & {$[1-4][5-9]$} \\
\hline $\mathbf{8}$ & $4,5-8,58$ ans & & & & {$[5-9]$} \\
\hline $\mathbf{7}$ & & & $>15$ ans & & {$[15-19]$} \\
\hline
\end{tabular}

Tabl. I - Classement en âge quinquennal de l'ensemble des sujets immatures après comparaison de leur maturation dentaire et osseuse:

* stature estimée sur relevés; $P$, périnatal.

\begin{tabular}{|c|c|c|c|c|c|}
\hline \multirow{2}{*}{ Individu } & \multicolumn{2}{|c|}{ Stade de fusion } & \multirow{2}{*}{$\begin{array}{c}\text { Classe } \\
\text { maturation }\end{array}$} & \multirow{2}{*}{$\begin{array}{c}\text { Âge } \\
\text { Schmitt } \\
\text { (2005) }\end{array}$} & \multirow{2}{*}{$\begin{array}{l}\text { Estimation } \\
\text { finale }\end{array}$} \\
\hline & clavicule & crête iliaque & & & \\
\hline 1 & totale & & $\geq 25$ ans & $>30$ & {$[>30]$} \\
\hline 2 & totale & totale & $\geq 25$ ans & $20-39$ & [25-39] \\
\hline 3 & totale & totale & $\geq 25$ ans & $20-49$ & [25-49] \\
\hline 4 & & totale & $\geq 25$ ans & & {$[>20]$} \\
\hline 6 & totale & totale & $\geq 25$ ans & $20-39$ & [25-39] \\
\hline 9 & & & $\geq 15$ ans & & {$[>15]$} \\
\hline 10bis & & & $\geq 15$ ans & & [>15] \\
\hline
\end{tabular}

Tabl. II - Estimation de l'âge au décès des adultes.

\begin{tabular}{|c|c|c|c|c|c|c|c|c|c|c|c|}
\hline \multirow{2}{*}{ Individu } & \multirow{2}{*}{$\begin{array}{c}\text { Classe } \\
\text { maturation }\end{array}$} & \multicolumn{7}{|c|}{ Critère observé sexe morphologique } & \multirow{2}{*}{$\begin{array}{c}\text { Sexe } \\
\text { morpho. }\end{array}$} & \multirow{2}{*}{$\begin{array}{l}\text { Sexe } \\
\text { DSP }\end{array}$} & \multirow{2}{*}{$\begin{array}{l}\text { Sexe } \\
\text { final }\end{array}$} \\
\hline & & \begin{tabular}{|l|} 
RP.1 \\
\end{tabular} & RP.2 & RP.3 & GS.1 & GS.2 & GS.3 & Ac & & & \\
\hline 1 & $A D$ & & & & & & & $\mathrm{~m}$ & $\mathrm{I}$ & $\mathrm{M}$ & M \\
\hline 2 & $A D$ & $\mathrm{~m}$ & $\mathrm{~m}$ & & $\mathrm{~m}$ & $\mathrm{~m}$ & $\mathrm{~m}$ & $\mathrm{~m}$ & M & M & M \\
\hline 3 & $A D$ & $\mathrm{f}$ & $\mathrm{i}$ & $\mathrm{f}$ & $\mathrm{f}$ & $\mathrm{f}$ & $\mathrm{f}$ & $\mathrm{f}$ & $\mathrm{F}$ & $\mathrm{F}$ & $\mathbf{F}$ \\
\hline 4 & $A D$ & & & & & & & & & & I \\
\hline 6 & $A D$ & $\mathrm{~m}$ & $\mathrm{~m}$ & & $\mathrm{~m}$ & $\mathrm{~m}$ & $\mathrm{~m}$ & $\mathrm{~m}$ & $\mathrm{M}$ & M & M \\
\hline 9 & TA & & & & & & & & & & $\mathrm{I}$ \\
\hline 10bis & TA & & & & & & & & & & $\mathrm{I}$ \\
\hline 7 & IM & $\mathrm{m}$ & $\mathrm{i}$ & & $\mathrm{m}$ & $\mathrm{m}$ & $\mathrm{m}$ & $\mathrm{m}$ & $\mathrm{I}$ & & I \\
\hline
\end{tabular}

Tabl. III - Détermination du sexe des adultes à partir de l'os coxal. Données crâniennes indicatives: $A D$, adulte; TA, taille adulte; IM, immature; $R P$, région préauriculaire; $G S$, grande incisure ischiatique; Ac, arc composé, le bord inférieur de l'os coxal et la longueur relative du pubis comparée à l'ischium n'ont été observés sur aucun squelette; $I$, indéterminé; $M$, masculin; F, féminin. appliqué la méthode probabiliste proposée par A. Schmitt (2005) relative aux caractères dégénératifs de la surface sacro-pelvienne iliaque de l'os coxal. L'individu 9 n'a pas pu être formellement identifié comme adulte (> 20 ans) en raison de sa faible représentation osseuse. Néanmoins, différents indices (voir description sépulture 9, p. 149) nous ont permis de le considérer comme un sujet de «taille adulte», donc d'au moins 15 ans. Nous avons classé dans cette même catégorie les restes osseux incinérés 10bis ne pouvant appartenir à un très jeune sujet (voir description sépulture 10bis, p. 155). L'estimation finale de l'âge des adultes en fonction des différents critères d'observation est présentée dans le tableau II.

\section{Diagnose sexuelle}

La détermination du sexe n'est fiable que chez les adultes et uniquement à partir des os coxaux. Nous avons appliqué la méthode morphoscopique classique (Bruzek, 2002). Conformément à ce que préconise cet auteur, l'individu a été sexé selon «la forme sexuelle» majoritaire (soit trois critères sur cinq). En raison de la conservation différentielle des squelettes de Chichery, cette analyse a été combinée avec une DSP (Murail et al., 2005). Les résultats de ces deux méthodes sont cohérents (tabl. III) ; dans un seul cas (individu 1), le sexe, indéterminable par la méthode morphoscopique, a été défini masculin par la DSP. 
À l'issue de notre analyse, nous avons identifié trois hommes et une femme (tabl. III). Le sexe du grand adolescent est laissé indéterminé (voir description sépulture 7, p. 143). La conservation osseuse des individus 4 (> 20 ans), 9 et 10bis («taille adulte») n’a permis aucune diagnose sexuelle à partir des os coxaux. Le faible effectif de la nécropole de Chichery ne nous autorise pas à appliquer le protocole proposé par P. Murail, J. Bruzek et J. Braga (1999) d'une diagnose sexuelle secondaire intrapopulationnelle à partir des mesures extrapelviennes. Les données de Chichery seront prises en compte dans une étude anthropologique plus large sur la population déterminée contemporaine et appartenant au même groupe culturel (travaux en cours). Cette démarche permettra éventuellement de sexer ces deux adultes indéterminés.

\section{Caractérisation morphométrique, pathologies, stress biologiques et variations anatomiques non métriques}

Une liste de 123 mesures crâniennes et infracrâniennes (Bräuer, 1988) par individu adulte a été établie afin de définir la morphologie de chaque sujet: stature (Trotter,

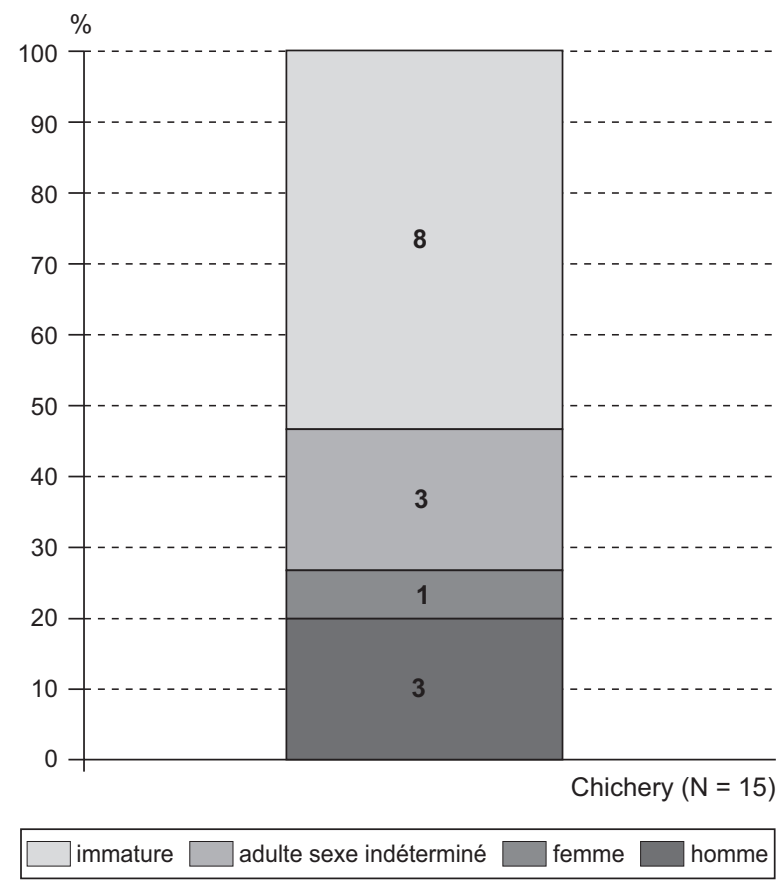

Fig. 43 - Composition par âge et par sexe de l'ensemble de la nécropole de Chichery. Dans l'hypothèse de trois ensembles, les sujets immatures sont presque tous localisés sur la file sud-est, accompagnés d'un seul adulte.
Gleser, 1958; Cleuvenot, Houët, 1993), capacité crânienne, robustesse des os longs.

La caractérisation biologique individuelle a également été abordée par le biais de la paléopathologie: nous avons analysé les atteintes osseuses arthrosiques et enthésopathiques (Villote, 2006) et les atteintes dentaires. L'usure (Smith, 1984), la maladie parodontale, le dépôt de tartre (Brothwell, 1965) et les éventuelles lésions carieuses ont été cotées selon trois critères: les différents stades de sévérité, les dents atteintes et les faces concernées.

En plus de ces informations, nous nous sommes focalisés sur le repérage systématique des hypoplasies linéaires de l'émail dentaire (cotées en fonction de la sévérité de la lésion et de sa distribution sur la couronne: tiers occlusal, moyen et cervical) et la présence de cribra orbitalia ou d'hyperostose poreuse afin d'identifier d'éventuelles périodes de stress subies par chaque individu.

Enfin, nous avons coté de façon systématique une série de variations anatomiques non métriques crâniennes (Hauser, De Stefano, 1989; Gemmerich Pfister, 1999), dentaires (Scott, Turner II, 1997) et infracrâniennes (Finnegan, 1978; Saunders, 1978). Ces marqueurs permettront de discuter d'éventuels regroupements familiaux.

\section{CARACTÉRISATION BIOLOGIQUE DES DÉFUNTS}

\section{Composition de la population par âge et sexe}

La population mise au jour à Chichery est composée de treize individus inhumés en sépultures primaires individuelles. À ce décompte, nous ajoutons les restes d'un enfant décédé en période périnatale découverts parmi les restes osseux de la femme 3 (dénommée 3bis) et les restes incinérés que nous avons appelés 10bis (fig. 43).

Les jeunes sujets se répartissent dans toutes les classes d'âge à l'exception de la classe des [10-14] ans (tabl. IV). Les méthodes d'estimation de l'âge des adultes ne permettent pas une répartition aussi précise des individus en classes d'âge. À cette limite théorique s'est rajouté l'état de conservation de squelettes très inégalement représentés. Trois adultes (2, 3 et 6 ) ont un âge formellement inférieur à 50 ans; nous n'avons pas pu préciser l'âge des sujets 1 (>30 ans) et 4 (> 20 ans) ; les individus 9 et 10bis, définis comme des sujets de «taille adulte» ont ici été compris dans l'échantillon adulte.

Sur les sept individus composant notre échantillon adulte, quatre ont pu faire l'objet d'une diagnose sexuelle (tabl. V) : l'individu 3 est une femme, les individus 1, 2 et 6 


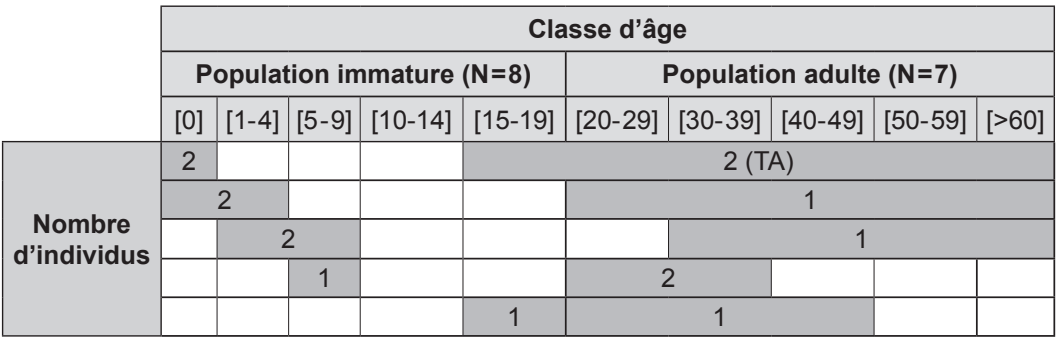

\begin{tabular}{|c|c|c|c|c|}
\hline Individu & Squelette & $\begin{array}{c}\text { Classe } \\
\text { maturation }\end{array}$ & Sexe & Âge \\
\hline $\mathbf{3 b i s}$ & oui & IM & & {$[\mathrm{P}]$} \\
\hline $\mathbf{1 1}$ & oui & IM & & {$[0]$} \\
\hline $\mathbf{1 2}$ & oui & IM & & {$[0][1-4]$} \\
\hline $\mathbf{1 0}$ & oui & IM & & {$[0][1-4]$} \\
\hline $\mathbf{1 3}$ & non & IM & & {$[1-4][5-9]$} \\
\hline $\mathbf{5}$ & non & IM & & {$[1-4][5-9]$} \\
\hline $\mathbf{8}$ & oui & IM & & {$[5-9]$} \\
\hline $\mathbf{7}$ & oui & IM & & {$[15-19]$} \\
\hline $\mathbf{9}$ & oui & TA & I & {$[>15]$} \\
\hline $\mathbf{1 0 b i s}$ & oui & TA & I & {$[>15]$} \\
\hline $\mathbf{4}$ & oui & AD & I & {$[>20]$} \\
\hline $\mathbf{1}$ & oui & AD & M & {$[>30]$} \\
\hline $\mathbf{2}$ & oui & AD & M & {$[25-39]$} \\
\hline $\mathbf{6}$ & oui & AD & M & {$[25-39]$} \\
\hline $\mathbf{3}$ & oui & AD & F & {$[25-49]$} \\
\hline
\end{tabular}

\begin{tabular}{|c|c|c|c|c|c|c|c|}
\hline \multirow{2}{*}{ Individu } & \multirow{2}{*}{ Sexe } & \multirow{2}{*}{$\begin{array}{c}\text { Stature } \\
(\mathbf{m})\end{array}$} & \multirow{2}{*}{$\begin{array}{c}\text { Os } \\
\text { concerné }\end{array}$} & \multicolumn{4}{|c|}{ Indice de robustesse } \\
\cline { 5 - 8 } & & humérus & ulna & radius & fémur \\
\hline $\mathbf{1}$ & $\mathrm{M}$ & $1,685 / 1,642$ & radius/ulna & & 15,1 & 17,1 & \\
\hline $\mathbf{2}$ & $\mathrm{M}$ & 1,569 & fémur & 21,2 & 18,6 & 18,9 & 20,6 \\
\hline $\mathbf{3}$ & $\mathrm{F}$ & 1,503 & fémur & 18,5 & 15,7 & 17,2 & 18,7 \\
\hline $\mathbf{4}$ & $\mathrm{I}$ & & & & & & \\
\hline $\mathbf{6}$ & $\mathrm{M}$ & 1,626 & fémur & 19,1 & & 18,6 & 19,9 \\
\hline $\mathbf{9}$ & $\mathrm{I}$ & & & & & & \\
\hline $\mathbf{1 0 b i s}$ & $\mathrm{I}$ & & & & & & \\
\hline
\end{tabular}

Tabl. VI - Stature et indices de robustesse des adultes: $M$, masculin; F, féminin; I, indéterminé.

Tabl. V - Récapitulatif de la composition par âge et par sexe de la population inhumée: IM, immature; TA, taille adulte; $A D$, adulte; $M$, masculin; $F$, féminin; I, indéterminé; $P$, périnatal.

Tabl. IV - Répartition par classes d'âge de la population immature et adulte. sont des hommes. Cet apparent déséquilibre ne peut en aucun cas être interprété comme significatif, compte tenu des trois sujets de sexe indéterminé (4, 9 et 10bis).

\section{Morphologie}

Les données morphométriques concernant les sept individus adultes sont rares et éparses au vu de leurs inégales conservations osseuses. Les résultats que nous donnons ici de la stature, la morphologie crânienne et infracrânienne ont, dans ce contexte, un intérêt purement descriptif.

La stature n'a pu être estimée que pour les individus 1, 2 , 3 et 6 (tabl. VI). Nous avons ici privilégié l'équation de régression calculée à partir de la longueur du fémur, reconnue comme plus fiable. La stature de l'individu 1 a été calculée à partir du radius et de l'ulna, en l'absence du fémur. La stature moyenne de 1,60 m calculée à partir de ces quatre seuls individus n'est de toute évidence qu'indica- 
Tabl. VII - Indices crâniens et infracrâniens calculés pour les adultes: $M$, masculin; F, féminin; I, indéterminé; FPT, frontopariétal transverse; FT, frontal transverse; HLa, hauteur sur largeur; HLo, hauteur sur longueur; $M H$, mixte de hauteur; O, orbitaire; $M$, mandibulaire, P, palatin; CC, capacité crânienne.

\begin{tabular}{|c|c|c|c|c|c|c|c|c|c|c|c|c|c|}
\hline \multirow{3}{*}{ Individu } & \multirow{3}{*}{ Sexe } & \multicolumn{9}{|c|}{ Indice crânien } & \multicolumn{3}{|c|}{ Indice infracrânien } \\
\hline & & \multirow{2}{*}{ FPT } & \multirow{2}{*}{ FT } & \multirow{2}{*}{ HLa } & \multirow{2}{*}{ HLo } & \multirow{2}{*}{ MH } & \multirow{2}{*}{0} & \multirow{2}{*}{ M } & \multirow{2}{*}{$\mathbf{P}$} & \multirow{2}{*}{ CC } & \multicolumn{2}{|c|}{ fémur } & \multirow{2}{*}{$\begin{array}{c}\text { tibia } \\
\text { cnémie }\end{array}$} \\
\hline & & & & & & & & & & & pilastrie & platymérie & \\
\hline 1 & $\mathrm{M}$ & & & & & & & large & & & faible & fort & faible \\
\hline 2 & M & large & divergent & moyen & haut & haut & & étroit & & moyen & moyen & faible & moyen \\
\hline 3 & $\mathrm{~F}$ & large & intermédiaire & & & & étroit & étroit & & & faible & fort & faible \\
\hline 4 & 1 & & & & & & & & & & & & \\
\hline 6 & $\mathrm{M}$ & & & & & & & & étroit & & faible & fort & moyen \\
\hline 9 & 1 & & intermédiaire & & & & & étroit & & & & & \\
\hline 10bis & $\mathrm{I}$ & & & & & & & & & & & & \\
\hline
\end{tabular}

tive. Notons que l'unique femme présente la stature la plus faible.

Le calcul des indices crâniens n'autorise aucune observation générale: la majorité n'ayant pu être appliquée qu'à un seul squelette, les résultats présentés dans le tableau VII font office de récapitulatif des informations individuelles disponibles.

La morphologie des fémurs et tibias a pu être décrite pour les individus 1, 2, 3 et 6 . Ces quatre individus présentent globalement un faible développement du pilastre fémoral. Dans trois cas sur quatre, cet os se caractérise également par une diaphyse fortement aplatie dans la région sous-trochantérienne. La diaphyse du tibia présente, quant à elle, un aplatissement moyen à faible au niveau du foramen nourricier.

Les indices de robustesse qui ont pu être calculés témoignent d'une relative homogénéité (tabl. VI). L'individu 2 présente la robustesse la plus importante pour chaque os concerné: humérus, ulna, radius et fémur.

Paléopathologie: atteintes dentaires, atteintes osseuses arthrosiques et enthésopathiques et indicateurs de stress

\section{État buccodentaire}

$\mathrm{Au}$ total, 193 dents permanentes et 18 déciduales ont été observées sur neuf individus (n'ont pu être observés les individus 5, 11, 12, 13, 3bis et 10bis). L'usure dentaire générale est faible et concerne les dents permanentes et déciduales (individu 8). En moyenne, les incisives et les premières molaires sont les plus atteintes, bien que la sévérité dépasse rarement l'apparition d'îlots dentinaires, parfois confluents, mais avec persistance d'un pourtour d'émail. Les troisièmes molaires sont logiquement les moins touchées et présentent, en moyenne, une usure très faible à nulle. Le sujet 1 se distingue nettement du reste des individus par une usure particulièrement marquée jusqu'à l'abrasion coronale totale de nombreuses dents, y compris les troisièmes molaires. Sur les 211 dents observées, seules cinq permanentes, réparties chez trois individus (1, 3 et 7), sont cariées. Ces lésions ne concernent que les dents postérieures et atteignent le collet ou la couronne de la dent. L'importance de l'atteinte est variable selon l'individu. Les 133 dents permanentes observées in situ (positionnées dans leurs alvéoles mandibulaires ou maxillaires) montrent rarement une atteinte parodontale. Cela ne concerne que les individus 1, 6 et 9 qui présentent une légère résorption de l'os alvéolaire. Enfin, le dépôt de tartre, qui ne concerne que les dents permanentes, touche préférentiellement l'arcade inférieure. La sévérité reste néanmoins modérée à nulle.

En résumé, l'état buccodentaire des individus de Chichery est globalement bon. L'usure est faiblement prononcée, les lésions carieuses et la maladie parodontale sont rares, et le dépôt de tartre modéré. Sur l'ensemble de ces critères, l'homme 1 paraît se démarquer par des atteintes plus sévères.

\section{Atteintes osseuses arthrosiques et enthésopathiques}

Les adultes 1, 2, 3, 4, 6 et le grand adolescent 7 ont permis de coter entre un et huit sites clefs d'observation du squelette appendiculaire permettant la description du développement des enthèses (Villotte, 2006). Aucune modification de ces insertions fibrocartilagineuses n'a été observée dans la majorité des cas. Quelques remaniements de faible sévérité ont été détectés sur les sujets 1, 2 et 3, principalement sur les membres supérieurs (facette supérieure et moyenne de l'humérus des sujets 2 et 3 , la tubérosité du 
Tabl. VIII - Nombre de dents atteintes d'hypoplasies linéaires de l'émail dentaire par individu: AD, adulte; IM, immature; $M$, masculin; $F$, féminin; $I$, indéterminé.

\begin{tabular}{|c|c|c|c|c|}
\hline Individu & $\begin{array}{c}\text { Classe } \\
\text { maturation }\end{array}$ & Sexe & $\begin{array}{c}\text { Dents } \\
\text { atteintes }\end{array}$ & $\begin{array}{c}\text { Dents } \\
\text { observées }\end{array}$ \\
\hline $\mathbf{1}$ & $\mathrm{AD}$ & $\mathrm{M}$ & 4 & 7 \\
\hline $\mathbf{2}$ & $\mathrm{AD}$ & $\mathrm{M}$ & 5 & 30 \\
\hline $\mathbf{3}$ & $\mathrm{AD}$ & $\mathrm{F}$ & 1 & 30 \\
\hline $\mathbf{4}$ & $\mathrm{AD}$ & $\mathrm{I}$ & 8 & 18 \\
\hline $\mathbf{6}$ & $\mathrm{AD}$ & $\mathrm{M}$ & 6 & 21 \\
\hline $\mathbf{7}$ & $\mathrm{IM}$ & & 3 & 31 \\
\hline $\mathbf{8}$ & $\mathrm{IM}$ & & 9 & 9 \\
\hline $\mathbf{9}$ & $\mathrm{AD}$ & $\mathrm{I}$ & 5 & 26 \\
\hline & & Total & $\mathbf{4 1}$ & $\mathbf{1 7 2}$ \\
\hline
\end{tabular}

Tabl. IX - Fréquence des dents atteintes par au moins une hypoplasie linéaire de l'émail dentaire (HLED) en fonction de leur position sur l'arcade.

\begin{tabular}{|c|c|c|c|c|c|c|c|c|c|c|c|c|c|c|c|c|}
\hline \multirow[b]{2}{*}{ Dent supérieure } & \multicolumn{8}{|c|}{ Hémi-arcade droite } & \multicolumn{8}{|c|}{ Hémi-arcade gauche } \\
\hline & M3 & M2 & M1 & $\mathrm{P} 2$ & $\mathrm{P} 1$ & $\mathrm{C}$ & 12 & 11 & 11 & 12 & $\mathrm{C}$ & $\mathrm{P} 1$ & $\mathrm{P} 2$ & M1 & M2 & M3 \\
\hline Dent observée & 5 & 8 & 7 & 6 & 5 & 7 & 4 & 5 & 7 & 5 & 7 & 4 & 6 & 7 & 6 & 7 \\
\hline HLED présente & 0 & 3 & 1 & 1 & 0 & 3 & 2 & 1 & 6 & 2 & 3 & 0 & 0 & 1 & 2 & 0 \\
\hline \multirow{2}{*}{$\begin{array}{l}\text { Fréquence } \\
\text { HLED (en \%) }\end{array}$} & 0 & 38 & 14 & 17 & 0 & 43 & 50 & 20 & 86 & 40 & 43 & 0 & 0 & 14 & 33 & 0 \\
\hline & 0 & 0 & 0 & 0 & 0 & 80 & 25 & 0 & 25 & 50 & 83 & 17 & 0 & 0 & 0 & 0 \\
\hline HLED présente & 0 & 0 & 0 & 0 & 0 & 4 & 1 & 0 & 1 & 3 & 5 & 1 & 0 & 0 & 0 & 0 \\
\hline Dent observée & 5 & 5 & 4 & 4 & 3 & 5 & 4 & 3 & 4 & 6 & 6 & 6 & 6 & 5 & 6 & 6 \\
\hline Dent inférieure & M3 & M2 & M1 & P2 & $\mathrm{P} 1$ & $\mathrm{C}$ & 12 & 11 & 11 & 12 & $\mathrm{C}$ & $\mathrm{P} 1$ & P2 & M1 & M2 & M3 \\
\hline
\end{tabular}

radius des sujets 1 et 2). Lorsque les deux côtés sont conservés, les observations sont bilatérales.

Ces mêmes sujets ne présentent aucune atteinte arthrosique sur l'ensemble des grandes articulations (épaule, coude, poignet, hanche, genou et cheville) ou sur le rachis. L'homme 1 fait exception en présentant de légères atteintes (épaule, poignet, hanche) et une importante arthrose rachidienne, avec ankyloses (vertèbres cervicales 3 et 4, vertèbre thoracique 8 et $8^{\mathrm{e}}$ côte gauche). Cet individu est en outre le seul de la nécropole à présenter plusieurs atteintes pathologiques (voir description sépulture 1, p. 123).

\section{Indicateurs de stress}

Les hypoplasies linéaires de l'émail dentaire n’ont été observées que sur les dents permanentes. Les huit individus (six adultes et deux sujets immatures) qui présentent une denture permanente observable (partielle ou complète) montrent tous au moins une hypoplasie (tabl. VIII). À l'exception de l'adolescent 7 , chaque individu présente au minimum une dent atteinte de deux hypoplasies (jamais plus). Ce constat évoque une faible succession de stress subie par les individus durant l'enfance. La fréquence des dents atteintes en fonction de leur position sur l'arcade démontre une atteinte des molaires faible (molaires supérieures) à nulle (molaires inférieures) (tabl. IX). Les dents antérieures, les canines inférieures et la première incisive supérieure gauche (observable dans une bonne proportion) présentent le taux d'atteinte le plus élevé.

La grande majorité des hypoplasies est située sur le tiers moyen $(68 \%)$ et le tiers cervical (28 \%) de la couronne, et concerne très largement $(82 \%)$ les incisives et les canines. Ces faits décrivent une période à laquelle les stress sont intervenus principalement entre 2,5 et 5 ans (Moorrees et al., 1963a et b).

Les atteintes osseuses que nous avons cotées (cribra orbitalia, hyperostose osseuse), pouvant également décrire des périodes de stress, sont bien plus rares. Sur les quatre surfaces orbitaires observables (2, 7, 9 et 10), seul l'adolescent 7 présente une cribra orbitalia légère et bien localisée. Parmi les neuf individus pour lesquels au moins un élément de la voûte crânienne était conservé (1, 2, 3, 4, 6, 7, 8, 9 et 10), aucun ne montre de trace d'hyperostose poreuse. 


\section{Variations anatomiques non métriques}

Nous présentons dans les tableaux X, XI et XII la liste des variations anatomiques non métriques (ou caractères discrets) crâniennes, infracrâniennes et dentaires cotées par individu et la fréquence de chaque caractère observé à Chichery. Ces fréquences doivent être pondérées en fonction du nombre d'individus pris en compte pour chaque calcul. En effet, la conservation différentielle des squelettes réduit nettement le nombre de cas observés et donne une image faussée des résultats (plusieurs fréquences particulièrement élevées sont parfois le fait d'un seul cas observable). Nous commenterons donc en priorité la fréquence des caractères observables dans une bonne proportion (qu'ils soient présents ou non) et focaliserons notre discours sur ceux dont un fort déterminisme génétique est suspecté, utiles à la recherche de regroupements familiaux (voir infra, p. 182). Notre démarche est ici avant tout descriptive. Nous pensons prématuré de comparer ce faible échantillon à d'autres populations. Cet aspect méthodologique pourra être abordé ultérieurement en regroupant ces données à d'autres ensembles funéraires du même type.

Nous avons testé statistiquement l'éventuel lien des variations anatomiques observées avec l'âge des individus à l'aide du test exact de Fisher bilatéral, au seuil de $5 \%$. Compte tenu des résultats de la diagnose sexuelle (une seule femme déterminée), aucun lien au sexe n'est recherché.

Les résultats concernant les variations crâniennes montrent une assez forte proportion d'individus présentant au moins un os surnuméraire sur l'ensemble de la voûte, observé le plus souvent bilatéralement (os suturaire sagittal, lambdoïde, à l'astérion, à l'incisure pariétale). Également bien représenté, le canal hypoglosse est bipartite chez trois individus (2, 8 et 10), dont deux immatures. Nous notons enfin que deux des quatre individus dont l'os frontal est conservé présentent une suture métopique totalement ouverte. Aucun des caractères crâniens observés n'est lié à l'âge des individus (tabl. X).

Les résultats de l'analyse des caractères infracrâniens appellent à peu de commentaires (tabl. XI). Sur les onze critères présents, dix ne concernent qu'un seul individu. Cinq individus sur six possèdent un calcanéus dont la surface articulaire talaire antérieure est bipartite.

Les restes dentaires, contrairement aux blocs craniofaciaux, ont été observés sur une grande proportion des individus de la nécropole. Les fréquences observées de caractères discrets dentaires sont donc plus pertinentes car plus représentatives (tabl. XII). Nous notons cinq variations représentées dans une forte proportion (> $50 \%$ ) : les formes en pelle des deuxièmes incisives supérieures, la présence de l'hypocône sur les premières et deuxièmes molaires supérieures, la présence d'une double cuspide linguale sur les deuxièmes prémolaires inférieures et l'absence de l'hypoconulide sur les premières molaires inférieures. À la liste de caractères dentaires présentés dans le tableau XII, nous ajoutons deux cas d'inclusion de la canine inférieure droite ou gauche (la femme 3 et l'homme 4). Le tubercule de Carabelli n'est présent que sur les premières molaires supérieures des sujets immatures observables (7, 8 et 10); aucun adulte ne présente ce trait. Cette différence est statistiquement significative au seuil de $5 \%(\mathrm{p}=0,0179)$. Aucun autre caractère dentaire ne montre de liaison à l'âge.

\section{CONGLUSION}

L'analyse anthropologique des individus mis au jour à Chichery révèle un nombre d'enfants de moins de 20 ans (huit sujets) comparable à celui des adultes (sept sujets). Les deux adultes de sexe indéterminé ne permettent pas de nous prononcer sur la proportion hommes/femmes inhumés dans la nécropole; notons néanmoins que les deux sexes sont représentés.

Dans l'ensemble, les atteintes pathologiques (pathologies dentaires, atteintes arthrosiques et enthésopathiques et indicateurs de stress) sont peu nombreuses et de faible intensité. Sur ces aspects, l'homme 1 semble se démarquer systématiquement.

Le faible effectif d'inhumés, d'une part, et la conservation différentielle, d'autre part, nous limitent dans l'interprétation des données morphométriques ou des fréquences des variations anatomiques non métriques obtenues. Ces données présentées ici ont avant tout un intérêt descriptif à l'échelle de l'individu. Incluses dans un échantillon plus large d'horizon chronoculturel similaire, ces informations participeront à la caractérisation de la population à laquelle appartient ce groupe ${ }^{21}$.

21. Cette caractérisation sera effectuée dans le cadre de la thèse en cours de A. Thomas à l'université Bordeaux-I Sciences Technologies, sous la direction de P. Chambon et de P. Murail. 
Tabl. $\mathbf{X}$ - Fréquence des variations anatomiques non métriques crâniennes de l'ensemble de l'échantillon et test de significativité (au seuil de 5 \%) en fonction de l'âge au décès: test, exact de Fisher (bilatéral); *, fort déterminisme suspecté; cases grises, caractère sélectionné pour la répartition spatiale; $N$ présent, nombre de cas où le caractère est présent; $N$ observé, nombre de cas où le caractère est observable.

\begin{tabular}{|c|c|c|c|c|c|c|c|c|}
\hline \multirow{2}{*}{ Caractère } & & \multirow{2}{*}{ Code } & \multirow{2}{*}{$\begin{array}{c}\mathrm{N} \\
\text { présent }\end{array}$} & \multirow{2}{*}{$\begin{array}{c}\mathbf{N} \\
\text { observé }\end{array}$} & \multirow{2}{*}{$\begin{array}{l}\text { Fréquence } \\
\text { en } \%\end{array}$} & \multirow{2}{*}{$\begin{array}{c}\text { Fisher exact } \\
\text { AD/IM }\end{array}$} & \multicolumn{2}{|c|}{ Individus concernés } \\
\hline & & & & & & & présent & absent \\
\hline os suturaire sagittal & & A1 & 1 & 2 & 50,00 & & 7 & 2 \\
\hline os suturaire coronal & & A2 & & 2 & 0,00 & & & $2 / 7$ \\
\hline os au bregma & & A3 & & 1 & 0,00 & & & 7 \\
\hline amincissement bipariétal & * & A4 & & 7 & 0,00 & & & $1 / 2 / 3 / 4 / 6 / 7 / 9$ \\
\hline foramen pariétal & & A5 & 4 & 4 & 100,00 & 1,0000 & $2 / 4 / 6 / 7$ & \\
\hline os fontanellaire au Lambda & & A6 & & 5 & 0,00 & & & $2 / 3 / 4 / 7 / 9$ \\
\hline os interpariétal & * & A7 & & 6 & 0,00 & & & $2 / 3 / 4 / 6 / 7 / 9$ \\
\hline os suturaire lambdoïde & * & A8 & 4 & 5 & 80,00 & 1,0000 & $2 / 3 / 6 / 7$ & 9 \\
\hline suture Mendosa & & A9 & 2 & 5 & 40,00 & & $2 / 6$ & $3 / 4 / 9$ \\
\hline foramen squameux inférieur & & A11 & 1 & 7 & 14,29 & 1,0000 & 4 & $2 / 3 / 6 / 7 / 8 / 9$ \\
\hline foramen squameux supérieur & & A12 & & 1 & 0,00 & & & 2 \\
\hline écaille du temporal bipartite & & A13 & & 1 & 0,00 & & & 2 \\
\hline os suturaire partie squameuse & & A14 & & & & & & \\
\hline articulation frontotemporale & * & A15 & & & & & & \\
\hline dépression supraméatique & & A18 & & 8 & 0,00 & & & $2 / 3 / 4 / 6 / 7 / 8 / 9 / 10$ \\
\hline suture squamomastoïdienne & & A19 & 1 & 4 & 25,00 & & 3 & $2 / 6 / 9$ \\
\hline foramen mastoïdien & & A20 & 3 & 5 & 60,00 & 0,4000 & $2 / 3 / 6$ & $7 / 9$ \\
\hline os suturaire occipitomastoïdien & & A22 & & 1 & 0,00 & & & 2 \\
\hline os à l'astérion & * & A23 & 3 & 3 & 100,00 & & $2 / 3 / 6$ & \\
\hline foramen pariétal inférieur & & A24 & & 4 & 0,00 & & & $1 / 2 / 6 / 7$ \\
\hline os pariétal bipartite & & A25 & & 6 & 0,00 & & & $1 / 2 / 3 / 4 / 6 / 7$ \\
\hline os à l'incisure pariétale & & A26 & 4 & 4 & 100,00 & 1,0000 & $2 / 3 / 6 / 7$ & \\
\hline torus du canal auditif externe & & A27 & & 8 & 0,00 & & & $2 / 3 / 4 / 6 / 7 / 8 / 9 / 10$ \\
\hline suture incisive & & A28 & 2 & 7 & 28,57 & 0,1429 & $7 / 8$ & $2 / 3 / 6 / 9 / 10$ \\
\hline os médiopalatin antérieur & & A29 & & 6 & 0,00 & & & $2 / 6 / 7 / 8 / 9 / 10$ \\
\hline épine osseuse sur palatin & & A57 & 5 & 7 & 71,43 & 0,1429 & $2 / 3 / 6 / 7 / 9$ & $8 / 10$ \\
\hline pont osseux sur palatin & & A30 & & 7 & 0,00 & & & $2 / 3 / 6 / 7 / 8 / 9 / 10$ \\
\hline torus palatin & & A31 & 1 & 6 & 16,67 & 1,0000 & 2 & $3 / 6 / 7 / 9 / 10$ \\
\hline torus maxillaire & * & A32 & 2 & 8 & 25,00 & 0,4643 & $2 / 6$ & $3 / 4 / 7 / 8 / 9 / 10$ \\
\hline tubercule pharyngien & & A33 & 2 & 6 & 33,33 & 0,4000 & $2 / 6$ & $3 / 7 / 8 / 10$ \\
\hline fossette pharyngienne naviculaire antérieure & & A34 & 1 & 6 & 16,67 & 1,0000 & 3 & $2 / 6 / 7 / 8 / 10$ \\
\hline tubercule précondylaire & & A35 & 1 & 6 & 16,67 & 1,0000 & 2 & $3 / 6 / 7 / 8 / 10$ \\
\hline canal basilaire médian & & A36 & & 5 & 0,00 & & & $2 / 3 / 6 / 8 / 10$ \\
\hline canal hypoglosse bipartite & * & A37 & 3 & 8 & 37,50 & 1,0000 & $2 / 8 / 10$ & $3 / 4 / 7 / 9 / 11$ \\
\hline canal condylaire intermédiaire & & A38 & 2 & 3 & 66,67 & 1,0000 & $4 / 11$ & 2 \\
\hline canal condylaire postérieur & & A39 & 2 & 3 & 66,67 & 1,0000 & $8 / 10$ & 11 \\
\hline incisure de la facette condylaire & & A56 & & 7 & 0,00 & & & 2/3/4/6/9/10/11 \\
\hline facette condylaire bipartite & & A40 & & 7 & 0,00 & & & 2/3/4/6/9/10/11 \\
\hline foramen occipital & & A42 & 2 & 3 & 66,67 & 0,3333 & $2 / 3$ & 7 \\
\hline canal craniopharyngien & & A43 & & 1 & 0,00 & & & 2 \\
\hline foramen de Vesale & & A44 & 1 & 3 & 33,33 & 0,3333 & 3 & $7 / 10$ \\
\hline foramen ovale vers lacerum & & A45 & & 3 & 0,00 & & & $3 / 7 / 10$ \\
\hline foramen ovale vers spinosum & & A46 & & 3 & 0,00 & & & $3 / 7 / 10$ \\
\hline foramen spinosum incomplet & & A47 & 2 & 3 & 66,67 & 1,0000 & $3 / 7$ & 10 \\
\hline épine ptérygo-alaire & & A48 & 3 & 4 & 75,00 & 1,0000 & $2 / 3 / 10$ & 7 \\
\hline pont ptérygo-alaire & & A49 & & 4 & 0,00 & & & $2 / 3 / 7 / 10$ \\
\hline épine ptérygospinale & & A55 & 3 & 4 & 75,00 & 1,0000 & $2 / 3 / 10$ & 7 \\
\hline pont ptérigospinal & & A50 & & 4 & 0,00 & & & $2 / 3 / 7 / 10$ \\
\hline foramen Huschke & & A54 & & 3 & 0,00 & & & $2 / 7 / 9$ \\
\hline suture métopique & & B1 & 2 & 6 & 33,33 & 1,0000 & $3 / 7$ & $2 / 4 / 9 / 10$ \\
\hline fissure métopique & & B2 & & 6 & 0,00 & & & $2 / 3 / 4 / 7 / 9 / 10$ \\
\hline sillon frontal & & B3 & & 6 & 0,00 & & & $2 / 3 / 4 / 7 / 9 / 10$ \\
\hline suture supranasale & & B4 & 5 & 6 & 83,33 & 1,0000 & $2 / 3 / 4 / 6 / 7$ & 9 \\
\hline trou supra-orbitaire, latéral et médian & * & B6 & & 8 & 0,00 & & & $1 / 2 / 3 / 4 / 6 / 7 / 9 / 10$ \\
\hline trou supratrochléaire & & B7 & & 7 & 0,00 & & & $2 / 3 / 4 / 6 / 7 / 9 / 10$ \\
\hline incisure supra-orbitaire médiane & & B8 & & 8 & 0,00 & & & $1 / 2 / 3 / 4 / 6 / 7 / 9 / 10$ \\
\hline incisure supratrochléaire & & B9 & 3 & 7 & 42,86 & 1,0000 & $2 / 7 / 9$ & $3 / 4 / 6 / 10$ \\
\hline fissure infra-orbitaire & & B11 & 1 & 1 & 100,00 & & 2 & \\
\hline trou infra-orbitaire accessoire & & B12 & & 1 & 0,00 & & & 2 \\
\hline os zygomatique bipartite & & B13 & & 8 & 0,00 & & & 1/2/3/4/6/7/8/9 \\
\hline tubercule marginal de l'os zygomatique & & B17 & 2 & 8 & 25,00 & 1,0000 & $3 / 9$ & $1 / 2 / 4 / 6 / 7 / 8$ \\
\hline foramen mentonnier multiple & & $\mathrm{C} 1$ & 1 & 9 & 11,11 & 1,0000 & 1 & $2 / 3 / 4 / 6 / 7 / 8 / 9 / 10$ \\
\hline foramen mentonnier accessoire & & $\mathrm{C} 2$ & & 8 & 0,00 & & & $1 / 2 / 3 / 4 / 7 / 8 / 9 / 10$ \\
\hline orifice du canal de Robinson & & C3 & & 7 & 0,00 & & & $1 / 2 / 3 / 4 / 6 / 7 / 9$ \\
\hline foramen rétromolaire & & $\mathrm{C} 4$ & 1 & 7 & 14,29 & 1,0000 & 6 & $1 / 2 / 3 / 4 / 7 / 9$ \\
\hline torus mandibulaire interne & * & C5 & & 8 & 0,00 & & & 1/2/3/4/6/7/8/9 \\
\hline foramen molaire & & C8 & 1 & 8 & 12,50 & 1,0000 & 2 & $1 / 3 / 4 / 6 / 7 / 8 / 9$ \\
\hline foramen de Serre & & C9 & 1 & 8 & 12,50 & 1,0000 & 4 & 1/2/3/6/7/8/9 \\
\hline pont mylo-hyoïdien & * & C10 & & 7 & 0,00 & & & $1 / 2 / 3 / 4 / 6 / 7 / 9$ \\
\hline condyle mandibulaire bipartite & & C11 & & 7 & 0,00 & & & $1 / 2 / 3 / 4 / 6 / 7 / 9$ \\
\hline tubercule subcondylaire & & $\mathrm{C} 12$ & & 7 & 0,00 & & & 1/2/3/4/6/7/9 \\
\hline
\end{tabular}


Tabl. XI - Fréquence des variations anatomiques non métriques infracrâniennes de l'ensemble de l'échantillon et test de significativité (au seuil de 5 \%) en fonction de l'âge au décès: test, exact de Fisher (bilatéral); $A D$, adulte; IM, immature; $N$ présent, nombre de cas où le caractère est présent; $N$ observé, nombre de cas où le caractère est observable.

\begin{tabular}{|c|c|c|c|c|c|c|c|c|}
\hline \multirow{2}{*}{ Os } & \multirow{2}{*}{ Caractère } & \multirow{2}{*}{ Code } & \multirow{2}{*}{$\begin{array}{c}\mathrm{N} \\
\text { présent }\end{array}$} & \multirow{2}{*}{$\begin{array}{c}\mathbf{N} \\
\text { observé }\end{array}$} & \multirow{2}{*}{$\begin{array}{c}\text { Fréquence } \\
\text { en } \%\end{array}$} & \multirow{2}{*}{$\begin{array}{c}\text { Fisher exact } \\
\text { AD/IM }\end{array}$} & \multicolumn{2}{|c|}{ Individus concernés } \\
\hline & & & & & & & présent & absent \\
\hline \multirow{9}{*}{$\frac{\pi}{\frac{\pi}{\pi}}$} & facette antérosupérieur bipartite & D1 & 1 & 7 & 14,29 & 1,0000 & 6 & $1 / 2 / 3 / 4 / 7 / 9$ \\
\hline & arc antérieur bifide & D2 & & 6 & 0,00 & & & $1 / 2 / 3 / 6 / 7 / 9$ \\
\hline & occipitalisation de l'atlas & D3 & & 7 & 0,00 & & & 1/2/3/4/6/7/9 \\
\hline & pont postérieur & D4 & & 7 & 0,00 & & & $1 / 2 / 3 / 4 / 6 / 7 / 9$ \\
\hline & pont latéral & D5 & & 5 & 0,00 & & & $2 / 3 / 6 / 7 / 9$ \\
\hline & pont rétro-articulaire & D6 & & 5 & 0,00 & & & $2 / 3 / 6 / 7 / 9$ \\
\hline & foramen transverse bipartite & D7 & & 3 & 0,00 & & & $2 / 3 / 9$ \\
\hline & foramen transverse incomplet & $\mathrm{D} 8$ & & 2 & 0,00 & & & $2 / 3$ \\
\hline & spina-bifida (arc postérieur bifide) & D9 & & 3 & 0,00 & & & $2 / 3 / 7$ \\
\hline \multirow{2}{*}{$\frac{n}{x}$} & foramen transverse bipartite & D10 & & 6 & 0,00 & & & $1 / 2 / 3 / 6 / 7 / 9$ \\
\hline & ossification ligament apical & D11 & & 6 & 0,00 & & & $1 / 2 / 3 / 6 / 7 / 9$ \\
\hline \multirow{2}{*}{ S } & foramen transverse bipartite & D12 & 1 & 4 & 25,00 & & 9 & $2 / 3 / 6$ \\
\hline & spina-bifida & $\mathrm{D} 13$ & & 5 & 0,00 & & & $1 / 2 / 3 / 7 / 9$ \\
\hline 5 & spina-bifida & D14 & & 4 & 0,00 & & & $1 / 2 / 3 / 7$ \\
\hline इ & spondylolyse & D15 & & 3 & 0,00 & & & $2 / 3 / 7$ \\
\hline \multirow{3}{*}{ 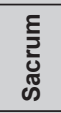 } & anomalie de nombre & D16 & & & & & & \\
\hline & spina-bifida & D17 & & & & & & \\
\hline & fusion sacrum coccyx & D18 & & & & & & \\
\hline \multirow{4}{*}{$\begin{array}{l}\frac{\pi}{5} \\
\frac{0}{\pi} \\
\text { 心 }\end{array}$} & os acromial & $\mathrm{F} 1$ & & 1 & 0,00 & & & 2 \\
\hline & foramen suprascapulaire & F2 & & 3 & 0,00 & & & $2 / 3 / 6$ \\
\hline & facette acromiale & F3 & & 1 & 0,00 & & & 2 \\
\hline & sillon circonflexe & $\mathrm{F} 4$ & & 3 & 0,00 & & & $1 / 2 / 3$ \\
\hline \multirow{2}{*}{$\begin{array}{l}\frac{0}{J} \\
\frac{0}{J} \\
\frac{\pi}{0}\end{array}$} & insertion en fosse ligament Cst-C & G1 & 1 & 4 & 25,00 & 1,0000 & 1 & $2 / 6 / 7$ \\
\hline & facette articulaire $\mathrm{Cr}-\mathrm{C}$ & G3 & 1 & 6 & 16,67 & 1,0000 & 1 & $2 / 3 / 4 / 6 / 7$ \\
\hline $\begin{array}{l}E \\
\text { Eे } \\
\text { ڤँ }\end{array}$ & perforation sternale & G5 & & & & & & \\
\hline \multirow{2}{*}{ 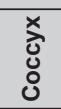 } & marque acétabulaire & E1 & 1 & 4 & 25,00 & & & $2 / 3 / 6$ \\
\hline & absence tubercule du pubis & E2 & & 1 & 0,00 & & & 2 \\
\hline \multirow{4}{*}{ 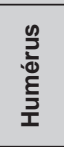 } & perforation olécranienne & $\mathrm{H} 1$ & & 4 & 0,00 & 1,0000 & 1 & $1 / 2 / 6 / 7$ \\
\hline & processus supracondylaire & $\mathrm{H} 2$ & & 8 & 0,00 & & & $1 / 2 / 3 / 4 / 6 / 7 / 8 / 12$ \\
\hline & insertion en fosse du grand pectoral & $\mathrm{H} 4$ & & 5 & 0,00 & & & $1 / 2 / 3 / 6 / 7$ \\
\hline & grand rond & H5 & & 5 & 0,00 & & & $1 / 2 / 3 / 6 / 7$ \\
\hline \multirow{4}{*}{ 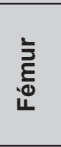 } & fosse Allen & L1 & & 3 & 0,00 & & & $2 / 3 / 6$ \\
\hline & facette de poirier & L2 & 1 & 4 & 25,00 & 1,0000 & 6 & $2 / 3 / 7$ \\
\hline & troisième trochanter & L3 & & 7 & 0,00 & & & $1 / 2 / 3 / 6 / 7 / 8 / 11$ \\
\hline & pilier latéral & L4 & 1 & 8 & 12,50 & 1,0000 & 1 & $2 / 3 / 6 / 7 / 8 / 10 / 11$ \\
\hline \multirow{2}{*}{$\begin{array}{l}\frac{\pi}{\overline{0}} \\
\overline{0} \\
0\end{array}$} & encoche latérale (vastus notch) & M1 & 1 & 4 & 25,00 & 1,0000 & 2 & $3 / 6 / 7$ \\
\hline & patella bipartite (emarginata) & M2 & & 4 & 0,00 & & & $2 / 3 / 6 / 7$ \\
\hline \multirow{2}{*}{$\frac{\pi}{\frac{\pi}{2}}$} & insertion du muscle soléaire en fosse & N1 & 1 & 9 & 11,11 & 0,4444 & 2 & $1 / 3 / 6 / 7 / 8 / 10 / 11 / 12$ \\
\hline & facettes d'accroupissement, médiale ou latérale & N2 & 1 & 3 & 33,33 & & 6 & $2 / 3$ \\
\hline \multirow{2}{*}{$\frac{\stackrel{n}{\Xi}}{\frac{\pi}{\sqrt{n}}}$} & os trigone & $\mathrm{P} 1$ & & 6 & 0,00 & & & $1 / 2 / 3 / 4 / 6 / 7$ \\
\hline & facette d'accroupissement médiale & $\mathrm{P} 2$ & & 6 & 0,00 & & & $1 / 2 / 3 / 4 / 6 / 7$ \\
\hline \multirow{2}{*}{ 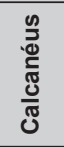 } & facette antérieure bipartite & P3 & 5 & 6 & 83,33 & 1,0000 & $1 / 2 / 3 / 4 / 7$ & 6 \\
\hline & emarginatus & P7 & & 6 & 0,00 & & & $1 / 2 / 3 / 4 / 6 / 7$ \\
\hline$\dot{0}$ & facette cunéonaviculaire & P6 & & & & & & \\
\hline ن : : & épine antérolatérale & P8 & & 4 & 0,00 & & & $1 / 2 / 3 / 6$ \\
\hline$E$ & facette proximale dédoublée & P5 & & 4 & 0,00 & & & $1 / 2 / 3 / 6$ \\
\hline
\end{tabular}


Tabl. XII - Fréquence des variations anatomiques non métriques dentaires de l'ensemble de l'échantillon et test de significativité (au seuil de 5 \%) en fonction de l'âge au décès: test, exact de Fisher (bilatéral); *, fort déterminisme suspecté; cases grises, caractère sélectionné pour la répartition spatiale; $N$ présent, nombre de cas où le caractère est présent; $N$ observé, nombre de cas où le caractère est observable.

\begin{tabular}{|c|c|c|c|c|c|c|c|c|c|c|}
\hline & \multirow{2}{*}{ Dent } & \multirow{2}{*}{\multicolumn{2}{|c|}{ Caractère }} & \multirow{2}{*}{ Code } & \multirow{2}{*}{$\begin{array}{c}\mathrm{N} \\
\text { présent }\end{array}$} & \multirow{2}{*}{$\underset{\text { observé }}{\mathrm{N}}$} & \multirow{2}{*}{$\begin{array}{l}\text { Fréquence } \\
\text { en } \%\end{array}$} & \multirow{2}{*}{$\begin{array}{c}\text { Fisher exact } \\
\text { AD/IM }\end{array}$} & \multicolumn{2}{|c|}{ Individu concerné } \\
\hline & & & & & & & & & présent & absent \\
\hline \multirow{31}{*}{ 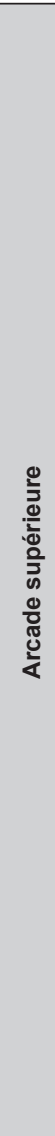 } & Ul1 & tubercule dentaire lingual & & UI1TD & & 7 & 0,00 & & & 2/3/4/6/7/8/9 \\
\hline & UI1 & sillon coronoradiculaire & & UI1SCR & & 7 & 0,00 & & & 2/3/4/6/7/8/9 \\
\hline & UI1 & incisive en pelle & * & UI1PEL & 1 & 7 & 14,29 & 0,2857 & 8 & $2 / 3 / 4 / 6 / 7 / 9$ \\
\hline & UI1 & double incisive en pelle & & UI1DPEL & & 7 & 0,00 & & & $2 / 3 / 4 / 6 / 7 / 8 / 9$ \\
\hline & $\mathrm{UI} 2$ & tubercule dentaire & & UI2TD & 1 & 8 & 12,50 & 1,0000 & 6 & $1 / 2 / 3 / 4 / 7 / 8 / 9$ \\
\hline & $\mathrm{UI} 2$ & sillon coronoradiculaire & & UI2SCR & 2 & 8 & 25,00 & 0,4643 & $6 / 8$ & $1 / 2 / 3 / 4$ \\
\hline & UI2 & incisive en pelle & & UI2PEL & 5 & 7 & 71,43 & 1,0000 & $2 / 3 / 6 / 7 / 8$ & $7 / 9$ \\
\hline & $\mathrm{UI} 2$ & double incisive en pelle & & UI2DPEL & & 7 & 0,00 & & & 2/3/4/6/7/8/9 \\
\hline & UCT & tubercule dentaire & & UCTD & 1 & 8 & 12,50 & 1,0000 & 6 & $1 / 2 / 3 / 4 / 7 / 8 / 9$ \\
\hline & UC & canine en pelle & & UCPEL & & 7 & 0,00 & & & $2 / 3 / 4 / 6 / 7 / 8 / 9$ \\
\hline & UC & double canine en pelle & & UCDPEL & & 8 & 0,00 & & & $1 / 2 / 3 / 4 / 6 / 7 / 8 / 9$ \\
\hline & UC & crête mésiale canine & & UCCM & & 4 & 0,00 & & & $2 / 3 / 7 / 8$ \\
\hline & UC & crête accessoire distale & & UCCAD & 1 & 4 & 25,00 & 1,0000 & 8 & $2 / 3 / 7$ \\
\hline & UP1 & tubercule marginal & & UP1TM & & 5 & 0,00 & & & $2 / 3 / 6 / 7 / 9$ \\
\hline & UP1 & nombre de racines & & UP1R & 3 & 6 & 50,00 & 1,0000 & $1 / 6 / 7$ & $3 / 4 / 9$ \\
\hline & UP1 & odontomes & & UP10 & & 5 & 0,00 & & & $2 / 3 / 6 / 7 / 9$ \\
\hline & UP2 & tubercule marginal & & UP2TM & & 6 & 0,00 & & & $2 / 3 / 4 / 6 / 7 / 9$ \\
\hline & UP2 & nombre de racine & & UP2R & & 7 & 0,00 & & & $1 / 2 / 3 / 4 / 6 / 7 / 9$ \\
\hline & UP2 & odontomes & & UP2O & & 6 & 0,00 & & & $2 / 3 / 4 / 6 / 7 / 9$ \\
\hline & UM1 & tubercules mésio-accessoires & & UM1TMA & 2 & 7 & 28,57 & 0,1429 & $8 / 10$ & $2 / 3 / 4 / 7 / 9$ \\
\hline & UM1 & parastyle & & UM1P & & 8 & 0,00 & & & $2 / 3 / 4 / 6 / 7 / 8 / 9 / 10$ \\
\hline & UM1 & métaconule & * & UM1M & 1 & 7 & 14,29 & 0,4286 & 8 & $2 / 3 / 4 / 7 / 9 / 10$ \\
\hline & UM1 & hypocane & & UM1H & 7 & 7 & 100,00 & 1,0000 & $2 / 3 / 4 / 7 / 8 / 9 / 10$ & \\
\hline & UM1 & extension de l'émail interradiculaire & * & UM1EE & & 8 & 0,00 & & & $1 / 2 / 3 / 4 / 6 / 7 / 8 / 9$ \\
\hline & UM1 & tubercule de Carabelli & * & UM1C & 3 & 8 & 37,50 & 0,0179 & $7 / 8 / 10$ & $2 / 3 / 4 / 6 / 9$ \\
\hline & UM2 & tubercules mésio-accessoires & & UM2TMA & 1 & 6 & 16,67 & 0,3333 & 8 & $2 / 3 / 4 / 7 / 9$ \\
\hline & UM2 & parastyle & & UM2P & & 7 & 0,00 & & & 1/2/3/4/7/8/9 \\
\hline & UM2 & métaconule & & UM2M & 1 & 6 & 16,67 & 0,3333 & 8 & $2 / 3 / 4 / 7 / 9$ \\
\hline & UM2 & hypocane & & $\mathrm{UM} 2 \mathrm{H}$ & 4 & 5 & 80,00 & 1,0000 & $3 / 7 / 8 / 9$ & 4 \\
\hline & UM2 & extension de l'émail interradiculaire & * & UM2EE & 1 & 5 & 20,00 & 0,2000 & 7 & $1 / 2 / 3 / 9$ \\
\hline & UM2 & tubercule de Carabelli & & UM2C & & 6 & 0,00 & & & $2 / 3 / 4 / 7 / 8 / 9$ \\
\hline \multirow{24}{*}{ 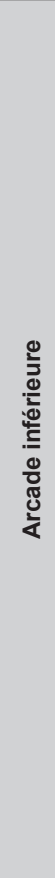 } & LI1 & incisive en pelle & & LI1PEL & & 5 & 0,00 & & & $2 / 3 / 7 / 8 / 9$ \\
\hline & LI2 & incisive en pelle & & LI2PEL & & 7 & 0,00 & & & 1/2/3/6/7/8/9 \\
\hline & LC & racine double canine & & LCRD & 1 & 6 & 16,67 & 1,0000 & 9 & $1 / 2 / 4 / 6 / 7$ \\
\hline & LC & crête accessoire distale & & LCCAD & & 3 & 0,00 & & & $3 / 4 / 7$ \\
\hline & LP1 & nombre de racines & & LP1R & & 6 & 0,00 & & & $1 / 2 / 4 / 6 / 7 / 8$ \\
\hline & LP1 & odontomes & & LP10 & & 6 & 0,00 & & & $1 / 2 / 3 / 4 / 6 / 7$ \\
\hline & LP1 & cuspide linguale multiple & * & LP1CLM & & 6 & 0,00 & & & $1 / 2 / 3 / 4 / 6 / 7$ \\
\hline & LP2 & odontomes & & LP2O & & 7 & 0,00 & & & 1/2/3/4/6/7/9 \\
\hline & LP2 & cuspide linguale multiple & * & LP2CLM & 4 & 5 & 80,00 & 1,0000 & $1 / 3 / 7 / 9$ & 4 \\
\hline & LM1 & triple racine & & LM1TR & & 7 & 0,00 & & & 1/2/4/6/7/8/9 \\
\hline & LM1 & protostylide (équi du parastyle) & & LM1P & & 8 & 0,00 & & & $2 / 3 / 4 / 6 / 7 / 8 / 9 / 10$ \\
\hline & LM1 & métaconulide & & LM1M & & 8 & 0,00 & & & $2 / 3 / 4 / 6 / 7 / 8 / 9 / 10$ \\
\hline & LM1 & hypoconulide & & LM1H & 3 & 7 & 42,86 & 0,4857 & $2 / 7 / 8$ & $3 / 4 / 9 / 10$ \\
\hline & LM1 & 4 cuspides & * & LM14C & 4 & 7 & 57,14 & & $3 / 4 / 9 / 10$ & $2 / 7 / 8$ \\
\hline & LM1 & fossette antérieure & & LM1FA & 1 & 7 & 14,29 & 0,4286 & 10 & $2 / 3 / 4 / 7 / 8 / 9$ \\
\hline & LM1 & entoconulide & & LM1E & & 7 & 0,00 & & & $2 / 3 / 4 / 7 / 8 / 9 / 10$ \\
\hline & LM1 & extension de l'émail interradiculaire & * & LM1EE & & 8 & 0,00 & & & $1 / 2 / 3 / 4 / 6 / 7 / 8 / 9 / 10$ \\
\hline & LM1 & groove pattern & & LM1GP & & 1 & 0,00 & & & 4 \\
\hline & LM2 & triple racine & & LM2TR & & 6 & 0,00 & & & $1 / 2 / 4 / 6 / 7 / 9$ \\
\hline & LM2 & protostylide (équi du parastyle) & & LM2P & & 7 & 0,00 & & & 1/2/3/4/6/7/9 \\
\hline & LM2 & métaconulide & * & LM2M & & 6 & 0,00 & & & $2 / 3 / 4 / 6 / 7 / 9$ \\
\hline & LM2 & hypoconulide & & LM2H & & 6 & 0,00 & & & $2 / 3 / 4 / 6 / 7 / 9$ \\
\hline & LM2 & extension de l'émail interradiculaire & * & LM2EE & 2 & 7 & 28,57 & 0,2857 & $2 / 7$ & $1 / 3 / 4 / 6 / 9$ \\
\hline & LM2 & groove pattern & & LM2GP & & 5 & 0,00 & & & $3 / 4 / 6 / 7 / 9$ \\
\hline
\end{tabular}




\section{UNE BRÈVE NOTE SUR LA CÉRAMIQUE DE SUR LES PÂTUREAUX}

Les tessons examinés sont issus de quatre sépultures distinctes: S2, S3, S8 et S9. L'inventaire initial mentionne des tessons dans deux sépultures supplémentaires que nous n'avons pas pu retrouver: il s'agit de S7 (1 tesson) et S13 (8 tessons). Pour leur description, nous renvoyons à la tombe correspondante (p. 143 et 158). Ajoutons que le nombre de tessons examinés pour la sépulture 8 est inférieur à celui du rapport de fouille (Delor, 1984).

La partie fouillée de la nécropole n'a livré aucune céramique complète, ni même aucune portion de vase qui puisse être envisagée comme un dépôt. Les tessons retrouvés l'ont été au sein des remplissages de différentes sépultures. Leur inclusion dans le comblement des tombes peut être l'œuvre d'animaux à galerie, notamment pour la sépulture 8 qui montre par ailleurs des signes de telles perturbations, mais elle témoigne plus vraisemblablement de matériel se trouvant en surface lors du creusement ou du remblai des tombes. À ce titre ces éléments sont logiquement plus anciens ou contemporains de la nécropole.

Sépulture 2: un tesson, au cour noir et aux surfaces beiges, comportant des inclusions de quartz (0,5-1 mm). La datation est indéterminée.

Sépulture 3: cinq tessons dont quatre appartiennent probablement au même individu. Le cœur est noir et les surfaces beiges et noires. Les inclusions de quartz sont fines $(0,5 \mathrm{~mm})$ et moyennement abondantes. La datation est indéterminée.

Sépulture 8: quarante tessons appartenaient certainement à un même individu. Ils sont épais de $8 \mathrm{~mm}$ en moyenne. De nombreuses cassures présentent une jointure de colombin. La pâte comporte d'abondantes esquilles d'os brûlé mesurant 1-2 mm. Quant aux couleurs, le cœur est noir, les surfaces et les marges orange. Le type de dégraissant ainsi que la couleur des surfaces sont caractéristiques des productions du groupe de Cerny.

Sépulture 9: six tessons de taille centimétrique dont un fragment de bord. La pâte est noire au cœur et la surface externe beige clair. Les inclusions sont constituées de quartz. Ces caractéristiques correspondent à de la céramique néolithique dont on ne peut préciser un horizon chronologique précis.

Aucun élément de forme ne figure parmi eux, à l'exception d'un fragment de bord non caractérisable dans la sépulture 9, ni aucun tesson décoré. Il s'agit dans tous les cas de tessons de céramique modelée, dont l'attribution au
Néolithique semble probable. Le site n'a pas livré de vestiges clairement assimilables à une occupation domestique, tant pour le Néolithique ancien que pour le Néolithique moyen; la surface explorée est cependant très réduite. Quelques caractères du vase le mieux représenté suggèrent un lien chronologique avec la nécropole mais, au-delà, la présence de ce matériel témoigne-t-elle d'activités en rapport avec l'occupation funéraire?

\section{L'INDUSTRIE LITHIQUE \\ EFFECTIFS ET MATIÈRES PREMIÈRES}

La série est composée de trente pièces dont la répartition figure dans le tableau XIII.

La matière première est constituée de silex pour l'essentiel. On en trouve trois types principaux:

- un silex à grain fin, brun à gris clair, sans zones de silicification imparfaite; il regroupe la grande majorité des pièces, soit 23 éléments ;

- un silex dit grenu, d'origine secondaire, de couleur grise, rugueux au toucher, avec des zones de silicification étendues; une seule pièce est fabriquée dans ce silex (sépulture $\left.8, \mathrm{n}^{\circ} 1\right)$;

- enfin, 3 éléments sont fabriqués dans un silex à grain très fin, très lisse au toucher, à patine beige crème à bleuté (sépulture $5, \mathrm{n}^{\circ} 1$; sépulture $10, \mathrm{n}^{\circ} 1$; sépulture $12, \mathrm{n}^{\circ} 1$ ).

À cela s'ajoutent une esquille de quartz (sépulture 5, $n^{\circ} 9$ ) et deux autres, brûlées, en silex indéterminé.

Les deux premiers types de silex devraient être d'origine sinon locale, du moins régionale. En revanche, le troisième type n'est pas identifié, pour le moment, dans un périmètre proche. Seul un programme de prospection systématique pourra apporter des éléments de réponse à cette question de l'origine géographique et géologique des matières premières.

Les deux éléments hors sépulture consistent en un petit nucléus polyédrique (dimensions du dernier enlèvement réussi: $20 \mathrm{~mm}$ x $18 \mathrm{~mm}$ ) et une esquille brûlée. Compte

Tabl. XIII - Répartition du matériel lithique dans les sépultures.

\begin{tabular}{|l|c|c|c|c|c|c|c|c|c|c|}
\cline { 2 - 11 } \multicolumn{1}{l|}{} & S2 & S3 & S4 & S5 & S6 & S8 & S10 & S12 & $\begin{array}{c}\text { Hors } \\
\text { sépulture }\end{array}$ & Total \\
\hline Silex & 5 & 2 & 3 & 8 & 2 & 4 & 2 & 1 & 2 & 29 \\
\hline Quartz & & & & 1 & & & & & & 1 \\
\hline Total & $\mathbf{5}$ & $\mathbf{2}$ & $\mathbf{3}$ & $\mathbf{9}$ & $\mathbf{2}$ & $\mathbf{4}$ & $\mathbf{2}$ & $\mathbf{1}$ & $\mathbf{2}$ & $\mathbf{3 0}$ \\
\hline
\end{tabular}


Tabl. XIV - Inventaire du matériel lithique en contexte de sépulture à Chichery.

\begin{tabular}{|c|c|c|c|c|}
\hline Sépulture & $\begin{array}{l}\text { Nombre } \\
\text { d'objets }\end{array}$ & Type d'objet & $\begin{array}{l}\text { Matière } \\
\text { première }\end{array}$ & Position \\
\hline \multirow{5}{*}{2} & \multirow{5}{*}{5} & racloir sur éclat laminaire & silex grain fin & Sous l'omoplate droite, talon vers la tête. \\
\hline & & pic-ciseau & silex grain fin & $\begin{array}{l}\text { Sur le poignet gauche, perpendiculaire à l'axe du corps, tranchant vers } \\
\text { l'extérieur. }\end{array}$ \\
\hline & & armature de flèche tranchante & silex grain fin & \multirow{3}{*}{$\begin{array}{l}\text { Les } 3 \text { armatures sont groupées près de la cheville gauche, partie active vers } \\
\text { le bas : les hampes devaient être placées le long du mollet. }\end{array}$} \\
\hline & & armature de flèche perçante & silex grain fin & \\
\hline & & armature de flèche losangique & silex grain fin & \\
\hline \multirow{2}{*}{3} & \multirow{2}{*}{2} & éclat laminaire & silex grain fin & Près de la tête, à quelques centimètres du cou. \\
\hline & & lame & silex grain fin & Sur l'avant-bras droit, perpendiculaire à l'axe du corps. \\
\hline \multirow{3}{*}{4} & \multirow{3}{*}{3} & fragment proximal de lame & silex grain fin & \multirow{2}{*}{ Dans le remplissage, au niveau de la poitrine et de l'abdomen. } \\
\hline & & éclat & silex grain fin & \\
\hline & & fragment distal de denticulé & silex grain fin & Près du mollet gauche. \\
\hline \multirow{8}{*}{5} & \multirow{8}{*}{9} & armature de flèche tranchante & silex grain très fin & \multirow{4}{*}{$\begin{array}{l}\text { Les } 4 \text { armatures sont groupées près de la tête, partie active vers le haut : les } \\
\text { hampes devaient être placées à droite du corps, entre celui-ci et l'alignement } \\
\text { de pierres. }\end{array}$} \\
\hline & & armature de flèche tranchante & silex grain fin & \\
\hline & & armature de flèche tranchante & silex grain fin & \\
\hline & & armature de flèche tranchante & silex grain fin & \\
\hline & & pic-ciseau & silex grain fin & $\begin{array}{l}\text { À l'emplacement de l'avant-bras gauche, perpendiculaire à l'axe du corps, } \\
\text { tranchant vers l'intérieur. }\end{array}$ \\
\hline & & éclat en 2 morceaux (cassure ancienne) & silex grain fin & À proximité du ciseau, sur la poitrine. \\
\hline & & éclat & silex grain fin & Position indéterminée. \\
\hline & & éclat & quartz & Position indéterminée. \\
\hline \multirow{2}{*}{6} & \multirow{2}{*}{2} & éclat laminaire & silex grain fin & Sur l'épaule droite. \\
\hline & & lame & silex grain fin & Sous l'épaule gauche. \\
\hline \multirow{4}{*}{8} & \multirow{4}{*}{4} & éclat & silex grenu & Dans le remplissage. \\
\hline & & éclat & silex grain fin & Dans le remplissage. \\
\hline & & éclat & silex grain fin & Dans le remplissage. \\
\hline & & éclat & silex grain fin & Dans le remplissage. \\
\hline \multirow{2}{*}{10} & \multirow{2}{*}{2} & armature de flèche tranchante & silex grain très fin & Près de l'épaule droite, dans le remplissage. \\
\hline & & esquille brûlée & silex indéterminé & Dans le remplissage. \\
\hline 12 & 1 & coche sur lame & silex grain très fin & Dans le remplissage. \\
\hline
\end{tabular}

tenu de leur faible valeur informative, il n'en sera plus question par la suite.

Sur treize sépultures, on dénombre huit structures renfermant des éléments lithiques: 28 pièces (tabl. XIV). Contrairement à d'autres nécropoles, l'essentiel de ces objets semble en association étroite avec l'inhumé.

\section{STATUTS DES MOBILIERS LITHIQUES}

Dans ces sépultures, le dépôt lithique occupe une place prépondérante: c'est le type de mobilier le plus fréquemment associé au mort. La céramique est quasiment inexistante et, lorsqu'elle est présente, sa position, dans le remplissage, indique un autre statut que les outils et les armes. La parure et l'outillage en matière dure animale sont, quant à eux, complètement absents, sans qu'il soit pos- sible d'invoquer un problème de conservation différentielle eu égard à l'état, relativement bon, des os humains.

Cette situation est inhabituelle dans ce secteur: à l'Étang David (Chichery) et aux Noisats (Gurgy), si la céramique est rare, la parure est néanmoins bien représentée aux côtés de quelques éléments lithiques (Rottier et al., 2005; Pellet, 1978). Il en est de même à Macherin (Monéteau) et, plus au nord dans la vallée de l'Yonne, aux Sablons (Gron) où la place de l'armement et de l'outillage de pierre est loin d'être aussi prégnante (Müller et al., 1997). On peut encore élargir la remarque aux nécropoles de Passy (Duhamel et al., 1997), de Balloy (Mordant, 1997), etc.

Ce trait revêt donc, pour le moment, un caractère d'exception qui mérite d'être largement souligné: Sur les Pâtureaux est la seule nécropole dont le mobilier conservé est constitué pour l'essentiel de silex taillé. Toutefois, le 
statut des pièces au sein des tombes n'est pas homogène. En effet, sur les 28 pièces lithiques en contexte sépulcral, six sont en position aléatoire par rapport à l'inhumé: elles ne peuvent lui être associées car elles proviennent du remplissage. La position de deux autres n'est pas connue. Toutefois, vingt éléments, les plus nombreux, peuvent être clairement connectés à l'inhumé: d'une part, leur altitude est identique ou proche du niveau des os et ils sont, pour la plupart, inclus dans le volume de l'espace confiné; d'autre part, leur position revêt un caractère récurrent ou cohérent par rapport à la disposition du corps.

\section{ÉLÉMENTS LITHIQUES EN POSITION ALÉATOIRE PAR RAPPORT AUX INHUMÉS}

Les sépultures concernées sont minoritaires, au nombre de trois: il s'agit des sépultures 8, 10 et 12. Les pièces concernées sont assez banales: quatre éclats dans la sépulture 8, une esquille dans la sépulture 10 et une coche sur lame dans la sépulture 12, pièce sans doute intrusive.

On a exclu de ce lot, peut-être arbitrairement, l'armature de la sépulture 10, en position légèrement plus haute que celle du corps mais à proximité du bloc craniofacial.

Pour les autres, leur position aléatoire dans le remplissage des tombes indique qu'elles sont en association fortuite avec les sépultures. A priori, il ne s'agit pas d'éléments participant au rituel funéraire, que ce soit au moment des funérailles ou par la suite.

\section{ÉLÉMENTS LITHIQUES AU MÊME NIVEAU QUE LES INHUMÉS}

Il s'agit pour l'essentiel d'outils ou d'armes retouchés ou façonnés - armatures de flèche, deux pics-ciseaux, racloir, lames - parfois fragmentés. Les éclats simples, non retouchés, sont rares: quatre éléments seulement. On notera dès ce stade l'absence de tranchets, contrairement aux Noisats (Augereau, 2008), à Monéteau (Augereau et al., 2003) ou aux Sablons (Müller et al., 1997).

\section{ARMATURES DE FLÈCHE}

C'est l'élément le plus fréquent: huit armatures sur vingt pièces lithiques en lien avec le corps. Elles sont associées soit à un homme (sépulture 2), soit à des enfants (sépultures 5 et 10), comme il est habituel pour ce type d'objets. Leur disposition est néanmoins variée:
- dans la sépulture 2, les trois armatures sont groupées près de la cheville gauche, toutes orientées avec la partie active vers les pieds; on devine que les flèches étaient disposées en fagot, les hampes le long du mollet;

- à l'inverse, les quatre flèches de la sépulture 5 sont placées près de la tête, partie active vers le haut; les hampes devaient s'aligner le long du corps, à sa droite;

- l'armature tranchante de la sépulture 10 est difficile à orienter; on peut seulement dire qu'elle était placée à droite du bloc craniofacial, près de l'épaule.

\section{PICS-CISEAUX}

Les sépultures 2 et 5 , un homme et un enfant, sont comparables non seulement par la présence de flèches mais également par celle de pics-ciseaux dans des positions de surcroît tout à fait comparables. Si celui de la sépulture 5 est de plus petite dimension, comme à la mesure de l'enfant inhumé, les deux sont semblablement disposés: la partie active sur l'avant-bras gauche, le manche placé contre le bras du mort. De surcroît, ces pièces sont usagées - reprise du biseau, écrasement de la partie opposée - mais les tests tracéologiques montrent qu'elles n'ont pas servi dans leur dernier état de réfection.

\section{LAMES, ÉCLATS LAMINAIRES, RACLOIRS}

Ces éléments ont été regroupés car ils ont en commun le caractère laminaire (le racloir est également sur un éclat allongé) et certains présentent des positions récurrentes. Quatre d'entre eux (racloir de la sépulture 2, éclat laminaire et lame de la sépulture 6, éclat laminaire de la sépulture 3) sont tous situés au niveau des épaules, sur ou sous l'omoplate ou très près des vertèbres cervicales. Doit-on déduire de cette disposition, fréquente à Chichery et ne pouvant être considérée comme fortuite, l'existence d'un dispositif particulier?

La dernière lame est, quant à elle, positionnée sur l'avant-bras droit du sujet de la sépulture 3, perpendiculairement à l'axe des os.

Sur le plan technologique, on remarque également, lorsque les modes de détachement peuvent être déterminés, que ces pièces ont été débitées par percussion tendre, comme c'est le cas pour le racloir de la sépulture 2 et pour la lame de la sépulture 6. Pour les autres, si la technique n'est pas clairement assurée, on peut tout de même exclure un débitage par percussion dure: absence de cône incipient 
et d'esquille de débitage bulbaire, bulbe long et peu renflé, corniche parfois abrasée.

Pour finir, il convient de noter que ces éléments laminaires sont associés soit à une femme (sépulture 3), soit à des hommes adultes (sépultures 2 et 6). Dans les autres nécropoles, la distribution de ce type de pièces est aléatoire - hommes, femmes et enfants - et Chichery ne semble pas s'éloigner de ce schéma.

Pour résumer, on soulignera que:

- deux tombes, une d'adulte masculin et une d'enfant, se distinguent des autres par l'abondance et la diversité du mobilier lithique associé; ce dernier, dont la composition est proche dans les deux cas, contient notamment des armatures de flèche, un pic-ciseau, soit des éléments classés, dans une précédente publication, parmi les instruments remarquables ${ }^{22}$ (Augereau et al., 2003);

- les autres sépultures, dont une femme, un homme et des enfants, présentent un mobilier beaucoup plus banal - lames, éclats laminaires, racloir, etc. - qu'on peut qualifier d'outils communs (id., ibid.). Cette catégorie d'objets est traditionnellement distribuée dans n'importe quel type de tombe.

Ce cas de figure, avec sépultures d'homme et d'enfant comportant des instruments remarquables aux côtés d'inhumations d'individus d'âge et de sexe variables, associés à des outils communs, est assez classique. On le retrouve à Monéteau (Augereau et al., 2003), à Balloy (Mordant, 1997) et à Passy (Duhamel et al., 1997).

\section{ASPECTS GHRONOGULTURELS}

Trois éléments peuvent apporter des informations sur ces aspects: les armatures de flèche, les pics-ciseaux et les pièces laminaires par percussion tendre. L'ensemble de ces pièces indique une ambiance générale Néolithique moyen avec, toutefois, différentes composantes.

Parmi les armatures de flèche, on distingue deux grands types:

- des pièces tranchantes, parmi lesquelles on a classé l'armature tranchante avec l'apex vers le haut de la sépulture 2, compte tenu de ses caractères technotypologiques;

22. On regroupe dans cette catégorie les objets issus d'un façonnage élaboré mettant en œuvre, pour les objets en silex, une retouche stéréotypée, comme les tranchets ou les pics. Il s'agit aussi de pièces témoignant d'une fonction précise, comme les armatures de flèche, ou issues d'un façonnage élaboré et se caractérisant par leur rareté (hameçons, spatules «anthropomorphes» Cerny, tube en os d'oiseau, défenses de suidés utilisées...). elles peuvent être triangulaires (fig. 11, $\mathrm{n}^{\circ} 3$; fig. 23 , $\mathrm{n}^{\text {os }} 2$ et 4 ) ou trapézoïdales (fig. $11, \mathrm{n}^{\circ} 2$; fig. $17, \mathrm{n}^{\mathrm{o}} 4$; fig. $23, \mathrm{n}^{\text {os }} 1$ et 3 ) ;

- une pièce perçante, de grande taille, de morphologie losangique (fig. 11, $\mathrm{n}^{\mathrm{o}} 1$ ).

Les armatures tranchantes, dont deux au moins sont fabriquées à partir d'une lame (fig. 23, nos 1 et 3), présentent toutes des retouches bifaciales, obliques à rasantes. Ce caractère est l'un des éléments constitutifs du Cerny Barbuise, tel qu'il a été perçu dans l'enceinte et la nécropole de Balloy (Mordant, 1997). Celui-ci se singularise par la présence d'armatures de flèche tranchantes à retouche rasante, parfois envahissante (Augereau, 2004); il s'éloigne ainsi du Cerny éponyme, du Cerny du nord du Bassin parisien, du Cerny de l'Ouest et du groupe de Chambon qui, dans leurs versions domestiques, ne comportent que des armatures à retouche abrupte ou semi-abrupte. Toutefois, en contexte funéraire régional, ce type d'armature à retouche abrupte n'est pas rare et se rencontre dans diverses cultures: dans le Cerny, à Passy (Duhamel et al., 1997) et à Gron (Müller et al., 1997), et dans le Chasséen, à Monéteau (Augereau et al., 2003 et 2005). Les photographies publiées de la nécropole d'Escolives-Sainte-Camille montrent aussi, semble-t-il, quelques pièces s'en rapprochant (Duhamel, 2004).

Larmature losangique, en revanche, est inhabituelle. Sa morphologie rappelle les exemplaires attribués au Néolithique récent du Bassin parisien (Augereau et al., 2007), mais sa position dans la sépulture ne fait aucun doute quant à son association avec le défunt. S'il est fréquent, dans les nécropoles régionales, Cerny comme chasséennes, de trouver aux côtés des armatures tranchantes quelques pièces perçantes triangulaires (Passy, Gron, Monéteau), il s'agit là d'un cas rare mais non unique. En effet, à Gron, la sépulture 14 renfermait une armature losangique de dimensions plus modestes et, dans la sépulture 352, est mentionnée une armature losangique que nous n'avons pas pu examiner (Müller et al., 1997). Par ailleurs, ce type d'armature est bien connu dans le Chasséen de Chassey (Thévenot, 1991) et dans le Chasséen méridional (Binder, 1991).

Quant aux deux pics-ciseaux, ils sont tout à fait comparables à ceux qu'on retrouve en contexte d'enceinte du Cerny Barbuise à Balloy (Augereau, 2004). En particulier, ceux dont le biseau est façonné par des enlèvements transversaux ou axiaux de type «coup de tranchet» semblent propres au Cerny: on les connaît dans l'enceinte de Balloy, mais également en contexte funéraire dans la sépulture 17.3 de Passy (Duhamel et al., 1997), dans la sépulture 2 de Noyen-sur-Seine (Mordant, Mordant, 1978) et dans 
la sépulture 1 des Gours aux Lions à Marolles-sur-Seine (Mordant et al., 1970).

Enfin, les quelques éléments laminaires par percussion tendre découverts à Chichery peuvent s'inscrire aussi dans cet horizon Cerny. Bien qu'il ait été largement démontré que le Cerny est la période d'abandon des méthodes de débitage laminaire élaboré (Augereau, Bostyn, 1997), la présence de lames dans les sépultures de cette période est un fait récurrent: certaines tombes de Gron, de Passy, mais aussi d'Orville dans le Loiret (Simonin et al., 1997), contiennent des lames régulières, débitées par percussion tendre ou par percussion indirecte. De manière générale, presque toutes les nécropoles régionales, à l'exception de Balloy, en ont livré: Monéteau mais aussi Escolives-Sainte-Camille. N'oublions pas, non plus, que l'enceinte chasséenne de Beaumont, toute proche, se caractérise par une série de lames régulières (Prestreau, Thévenot, 1996; Prestreau, 2006). Dans le cas de Chichery, le fait marquant réside dans le caractère fragmentaire ou de dimensions réduites de ces pièces. Partout ailleurs en milieu funéraire, les lames sont de grande taille, le plus souvent entières et sans accident de débitage, tel le réfléchissement observé sur la lame de la sépulture 6 .

Pour résumer, l'analyse des principales pièces lithiques des sépultures de Sur les Pâtureaux montre qu'une majorité peut se rattacher sans problème au Cerny. Tel est le cas des armatures tranchantes à retouches bifaciales obliques à rasantes et du pic à extrémité biseautée par «coup de tranchet». L'armature losangique renverrait, quant à elle, à la sphère chasséenne. Cette situation, avec des éléments renvoyant à diverses entités culturelles, est assez fréquente en contexte funéraire Cerny, avec toutefois une exception notable, celle de Balloy. Mais généralement, ce sont les composantes nord-orientales qui prédominent: des armatures perçantes triangulaires et peut-être aussi des lames par percussion indirecte, d'obédience Rössen ou Épi-Rössen. La référence à une sphère chasséenne bourguignonne ou méridionale, si elle n'est pas exceptionnelle, est plus rare.

\section{CONGLUSION}

En guise de conclusion, les caractéristiques du mobilier lithique de la nécropole de Sur les Pâtureaux peuvent se résumer ainsi :

- le dépôt quasi exclusif d'objets lithiques associés aux défunts est un caractère original, la parure et les outils en matière dure animale étant complètement absents, la céramique rare et placée hors du volume de l'espace confiné;
- deux sépultures se distinguent, celle d'un homme et celle d'un enfant, par la présence d'instruments remarquables: nombreuses flèches, pics-ciseaux; les autres sépultures ne contenant que des outils communs ou étant sans mobilier;

- la récurrence de la position de certains outils communs, à caractère laminaire, tels des éclats laminaires, des lames, des racloir situés sur ou sous l'épaule, ne peut être fortuite; ces artefacts étaient-ils accrochés au vêtement ou placés dans un sac jeté sur l'épaule?

- la technotypologie des objets renvoie, d'une part, à l'entité Cerny et, d'autre part, au Chasséen bourguignon ou méridional; cette situation est habituelle dans les contextes funéraires locaux mais la représentation de la sphère nordorientale est généralement plus importante;

- la composante laminaire, fréquemment attestée en contexte funéraire régional, est certes représentée ici mais par des éléments de mauvaise facture ou fragmentés.

Pour finir, on soulignera de nouveau l'absence de tranchets à Chichery alors que ceux-ci existent dans presque toutes les autres nécropoles régionales: Monéteau, Passy, Gron, Balloy... À moins que les deux pics-ciseaux aient rempli, au sein du rituel funéraire, le même rôle symbolique, cultuel et fonctionnel.

\section{LA FONCTION DE L'OUTILLAGE LITHIQUE}

\section{INTRODUCTION}

L'étude tracéologique réalisée sur le gisement de Chichery avait pour objectif de déterminer l'utilisation des instruments lithiques associés aux sépultures qui composent cette nécropole ${ }^{23}$.

Il va de soi que la finalité de l'étude fonctionnelle n'a pas pour unique objectif de définir l'utilisation des outils. Les

23. La démarche tracéologique débute par l'enregistrement de tous les résidus organiques et inorganiques présents à la surface des pièces. Malheureusement, lors de la fouille il y a plus de 25 ans, aucune étude de ce type n'était envisagée; les pièces analysées avaient donc été nettoyées, les résidus éliminés. L'étape suivante est l'observation macroscopique et microscopique des traces d'usage. Pour mener à bien cette analyse avec le maximum de garanties, il est indispensable que toutes les pièces passent par un processus rigoureux de nettoyage. Pour enlever le sédiment qui adhère encore au matériel, nous utilisons exclusivement des solutions d'eau et de savon ou d'eau oxygénée $\left(\mathrm{H}_{2} \mathrm{O}_{2}\right)$, à température ambiante, en petites quantités. Concernant Chichery, l'absence de résidus calcaires nous a évité l'emploi de solutions acides (acide chlorhydrique: $\mathrm{H}_{3} \mathrm{OCl}$ ). L'observation des pièces a été réalisée en conjuguant une loupe binoculaire Olympus, dont le grossissement va de $10 \mathrm{x}$ à $90 \mathrm{x}$, et un microscope métallographique Olympus BH2, dont le grossissement varie de 50x à 400x. 
objectifs que nous poursuivons sont avant tout historiques. Ils s'insèrent dans une problématique plus large de caractérisation du mobilier associé aux morts, liant statut des objets dans la tombe et identité du défunt. Ce programme, initié avec la nécropole de Sur les Pâtureaux, portera sur tous les ensembles du début du Néolithique moyen.

L'outillage lithique analysé a subi de fortes altérations chimiques et mécaniques. Parmi ces altérations, il faut souligner l'apparition d'intenses patines et de lustrés de sol très prononcés. Nous leur devons la présence, sur diverses pièces, de nombreuses stries d'orientation chaotique, démontrant bien que nous sommes devant des altérations mécaniques.

Il est le plus souvent impossible d'observer clairement des traces d'usage diagnostiques: les altérations ont détruit ou masqué les traces générées par l'utilisation. Ceci se répète sur les nombreuses pièces que nous avons jugées non analysables et sur le peu d'outils sur lesquels nous avons pu observer de possibles traces d'utilisation par ailleurs douteuses.

\section{Les altérations et leurs répercussions sur les traces d'usure}

Les altérations les plus communes à Chichery sont, d'une part, un type de patine dit «lustré de sol» et, d'autre part, des modifications mécaniques ${ }^{24}$. Pour rendre plus compréhensibles les conséquences de ces différentes altérations ainsi que les résultats obtenus lors de l'analyse tracéologique, nous ferons une description sommaire de leurs caractéristiques et de leurs répercussions.

Pour ce qui est du «lustré de sol», nous ne connaissons pas, pour le moment, son origine. Cependant, quelques travaux expérimentaux ont permis de proposer qu'il pouvait être la conséquence d'une action chimique ou mécanique (Levi-Sala, 1993; Clemente, 1998). À l'échelle microscopique, ce lustré couvre toute la surface de la pièce mais plus spécifiquement les zones élevées de la microtopographie: arêtes, fil de tranchant et contour des écaillages générés lors de l'utilisation ou de la retouche. Ses caractères sont les suivants: trame semi-fermée et, dans quelques cas, compacte, légèrement bombée, épaisse et brillante.

24. Les altérations ont d'importantes répercussions, non seulement sur le développement des traces d'usure, mais aussi sur leur préservation et leur observation. Comprendre ces mécanismes d'altération permet de mieux observer et identifier les traces d'usure. Les résultats sont alors optimisés et leurs implications archéologiques mieux évaluées.
Les micropolis peu développés, engendrés par l'utilisation des instruments sur des matériaux de dureté souple, ne peuvent pas être différenciés de ce lustré. Les rares petits écaillages et le léger émoussé qui apparaissent lors d'un travail sur matériaux souples ne sont pas des éléments suffisants pour assurer que les pièces ont été utilisées. En effet, de telles traces peuvent avoir d'autres causes: taille, altérations d'origines diverses, stockage du matériel, etc. Ce type de lustré n'a pas de conséquences aussi graves dans le cas de traces provoquées lors du travail de matières dures ou de matériaux qui entraînent des micropolis très développés et étendus, comme ceux produits lors de la coupe des céréales. Le problème devient encore plus épineux lorsque diverses altérations se superposent, comme c'est le cas à Chichery.

Parmi les altérations les plus communes sur le matériel de Chichery, il faut mentionner la patine, qui a affecté considérablement la surface des pièces. Seul un éclat de la sépulture 8 ne présente pas de traces de patine. Dans les cas où la patine se situe seulement sur le fil des tranchants ou sur les arêtes, il est possible de détecter les micropolis les plus développés et compacts (et non ceux peu intenses ou de trame ouverte/semi-fermée engendrés par le travail de la viande, du poisson ou de la peau fraîche). Quand le degré de développement de cette patine est très élevé, la surface devient si altérée et si modifiée qu'il est pratiquement impossible que les micropolis résultant du travail d'un quelconque matériau soient conservés. C'est encore le cas du matériel de Chichery.

Une autre situation problématique est le fait des altérations mécaniques. Sur la surface de la majorité des pièces, nous avons enregistré de nombreuses stries d'orientation chaotique, ainsi que des zones de poli « $\mathrm{G}$ », provoquées par le frottement de ces pièces lithiques avec d'autres objets lithiques. Toutes ces modifications témoignent des altérations mécaniques intenses que ces pièces ont subies.

\section{Résultats de l'analyse tracéologique de Chichery}

Nous avons analysé un corpus de vingt-sept pièces, parmi lesquelles quatre présentent de possibles traces d'usage et cinq n'ont pas été utilisées, tandis que nous en avons considéré dix-huit comme non analysables, car les altérations qui les affectent ne nous permettent pas d'obtenir des critères diagnostiques clairs permettant d'affirmer si les pièces ont ou n'ont pas été utilisées.

En ce qui concerne les pièces utilisées, trois d'entre elles présentent des traces sur le fil du tranchant seulement. La dernière montre trois zones actives. 


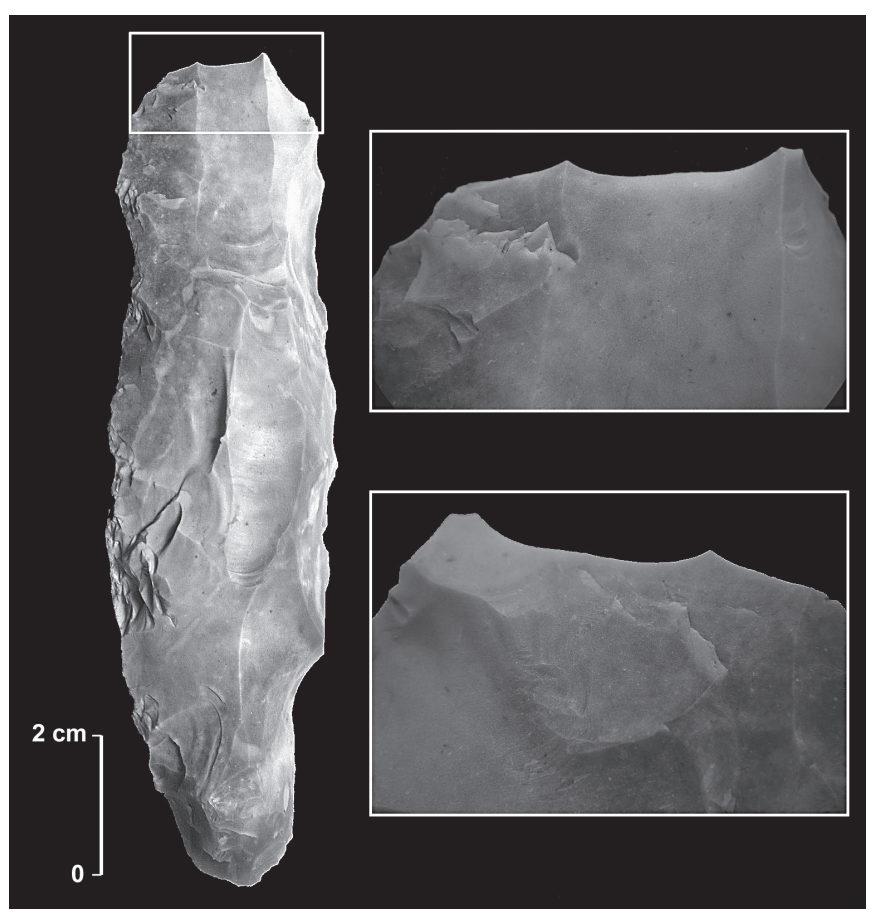

Fig. 44 - Pic de la sépulture 2 avec agrandissement de la partie active: on n’observe pas la moindre ébréchure (cliché:J. F. Gibaja Bao).

\section{Sépulture 2}

Nous avons analysé cinq pièces: un pic, deux armatures tranchantes, une armature perçante et un éclat large. Seul l'éclat présente un ensemble de traces que nous pensons peut-être correspondre à un usage type décharnement. Cette détermination est délicate au vu de la surface légèrement patinée et lustrée de la pièce. Le côté opposé est retouché et montre des micropolis de trame semi-fermée généralement liés au travail d'un matériau végétal de type bois. Ces traces sont parfois produites par le contact avec un manche.

D’un autre côté, bien que nous ayons jugé les trois armatures, tranchantes et perçantes, comme non analysables, nous avons considéré que la pièce identifiée comme un pic n'avait pas été utilisée. De toute façon, il est probable qu'aucune de ces pièces ne l'ait été. Si les armatures tranchantes et perçantes sont des pointes de projectile, dans le cas présent, elles ne présentent aucun type de fracture par impact.

Le cas du pic est identique. Ce qui paraît être la partie active ne présente pas la moindre modification d'utilisation, au moins au niveau macroscopique (fig. 44). Nous n'observons pas la moindre ébréchure. Nous entrevoyons deux possibilités: la pièce a été réalisée et déposée comme mobilier funéraire sans être utilisée, ou elle a bien été uti- lisée mais elle a été réaffûtée peu avant son dépôt dans la sépulture.

\section{Sépulture 3}

Nous avons étudié deux lames dont une est fragmentée à sa partie distale. Nous les avons classées parmi les pièces non analysables à cause du degré de patine et du lustré de sol. Les tranchants sont en bon état, sans ébréchures. Ceci nous conduit à envisager la possibilité que ces pièces n'aient pas été utilisées ou qu'elle l'aient été sur un matériau souple, qui génère peu d'ébréchures. Les altérations présentes nous empêchent de nous prononcer en faveur de l'une ou de l'autre hypothèse.

\section{Sépulture 4}

Nous avons analysé deux éclats et une lame fragmentée. Alors qu'un des éclats ne paraît pas avoir été utilisé et que la lame a été jugée non analysable, le deuxième éclat présente sur le côté droit une série d'ébréchures et des zones de micropolis de trame semi-fermée compacte qui nous font penser au raclage du bois. Le mauvais état de surface impose une certaine réserve.

\section{Sépulture 5}

Un total de neuf pièces a été analysé pour cette sépulture: un pic, quatre armatures tranchantes, trois éclats (dont un sur cristal de roche) et un possible petit fragment distal de lame. Comme dans la sépulture 2, les armatures tranchantes ne présentent pas de fractures d'impact et le pic ne montre pas la moindre ébréchure sur ce qui doit théoriquement être le tranchant actif. Dans le cas des éclats, il y a quelques petites ébréchures à certains points des tranchants. Il s'agit toutefois d'ébréchures non diagnostiques, qui pourraient tout aussi bien résulter d'altérations mécaniques.

\section{Sépulture 6}

Les deux lames mises au jour dans cette sépulture sont très patinées, raison pour laquelle nous les considérons comme non analysables. L'une d'entre elles est en si bon état qu'elle paraît ne pas avoir été utilisée, mais le degré de patine nous empêche d'être affirmatifs.

\section{Sépulture 8}

Nous avons analysé un total de quatre pièces issues de cette sépulture: trois éclats de taille réduite et un éclat allongé retouché sur un côté. L'un des éclats n'est pas usé, deux autres ne sont pas analysables. En revanche, l'éclat 
allongé montre sur deux côtés un ensemble de traces qui peuvent correspondre à une usure. Sur le côté gauche une superposition de traces correspond au travail de deux matériaux distincts: coupe de peau et coupe de céréales; sur le côté droit, retouché, apparaît un intense émoussé probablement produit par le grattage de la peau. Ce constat traduit le réemploi d'instruments conçus initialement pour une activité déterminée.

\section{Sépulture 10}

Cette sépulture n'a livré qu'une seule pièce s'apparentant à une armature tranchante de morphologie rectangulaire. De nouveau, cette pièce est très patinée et ne présente pas de fractures d'impact. Pour cette raison nous l'avons également jugée non analysable.

\section{Sépulture 12}

Dans cette dernière sépulture nous avons analysé un éclat comportant une petite encoche distale réalisée par retouche abrupte. C'est précisément dans cette zone que nous avons retrouvé des zones de micropolis compacts similaires à ce que génère le travail de matières dures animales comme l'os ou le bois de cervidé. Le diagnostic est fragilisé par le haut degré de patine de la pièce.

\section{CONCLUSIONS}

Les informations que nous avons extraites de l'échantillon réduit et mal conservé sont peu nombreuses. Nous pouvons toutefois éclairer certains aspects:

- l'outillage est intensément altéré. En raison du lustré de sol, de la patine et des altérations mécaniques, nous sommes amenés à classer la majorité des pièces comme non analysables ;

- quelques pièces comme les armatures tranchantes et perçantes, traditionnellement des projectiles en contexte néolithique, ne présentent aucun écaillage et plus spécifiquement de fractures d'impact. Cela implique-t-il que les pièces sélectionnées comme mobilier n'ont pas été utilisées? Nous avons déjà formulé une telle hypothèse pour d'autres contextes funéraires néolithiques, au nord-est de la péninsule Ibérique, où des armatures non utilisées figurent aussi dans le mobilier funéraire (Gibaja Bao, 2003);

- le cas est identique pour les probables pics. Le fil du tranchant théoriquement actif est intact pour les deux objets. Ceci nous permet d'envisager deux hypothèses: il s'agit soit de pièces réalisées ex professo pour être insérées dans la tombe, soit, plus probablement, de pièces utilisées préalablement mais réaffûtées à cette occasion. Un tel réaffûtage a pu faire disparaître les écaillages produits antérieurement et produire de nouveau un fil de tranchant en parfait état;

- parmi les rares pièces utilisées, diverses activités sont représentées: décharnement, coupe et grattage de la peau, coupe de végétaux et grattage de matière dure animale.

En dépit de l'état des pièces, on peut s'interroger sur un possible dualisme des assemblages mobilier, comprenant des outils utilisés et des outils réalisés ex professo pour accompagner les inhumés.

\section{VUE D'ENSEMBLE DE LA NÉCROPOLE \\ RECRUTEMENT DE LA POPULATION INHUMÉE}

La population de ce groupe de sépultures comprend quinze sujets. Malgré un nombre de sépultures fixé à treize, il reste difficile de ne pas considérer les tombes comme individuelles: on ne peut assurer que le sujet périnatal de la tombe 3 est bien «indépendant» de l'occupant principal de la sépulture; les restes incinérés que nous avons appelés 10bis sont nettement disjoints de l'inhumé sous-jacent.

L'analyse du recrutement (Masset, 1987) de la population inhumée à Chichery telle qu'elle a été découverte, se borne à un problème majeur: la non exhaustivité de la fouille. En effet, le décapage n'ayant pas été étendu très au-delà des tombes découvertes, nous ne sommes pas en mesure d'affirmer que les quinze sujets mis au jour constituent l'intégralité des individus effectivement inhumés dans la nécropole. Dès lors, toute interprétation en terme de recrutement est tronquée. Si la comparaison de cet échantillon de quinze individus aux données théoriques nous permet de discuter de sa compatibilité avec un recrutement naturel, rien, en revanche, ne nous dira si cet échantillon est représentatif de l'ensemble de la nécropole et, par conséquent, si nos résultats décrivent l'image réelle du recrutement de l'intégralité de l'espace funéraire.

La construction de notre table de mortalité a été soumise au principe directeur de la conformité maximale de nos paramètres paléodémographiques au schéma de mortalité archaïque (ou préjennérienne), proposé par P. Sellier (1996). Dans le cas d'alternatives, tels les quatre individus pouvant appartenir à deux classes d'âge (tabl. IV), nous choisissons donc systématiquement l'hypothèse qui se conforme le mieux à ce schéma de mortalité archaïque. Si, malgré cela, des écarts avec ce schéma existent, nous ne pourrons pas alors les attribuer à la méthode. La réparti- 
Tabl. XV - Répartition finale de la population immature par classes d'âge selon le principe de minimalisation des anomalies démographiques: AD, adulte (d'après Sellier, 1996).

\begin{tabular}{|c|c|c|c|c|c|c|}
\cline { 2 - 7 } \multicolumn{1}{c|}{} & \multicolumn{6}{c|}{ Classe d'âge } \\
\cline { 2 - 7 } \multicolumn{1}{c|}{} & {$[0]$} & {$[1-4]$} & {$[5-9]$} & {$[10-14]$} & {$[15-19]$} & $A D$ \\
\hline $\begin{array}{c}\text { Nombre } \\
\text { d'individus }\end{array}$ & 3 & 3 & 1 & 0 & 1 & 7 \\
\hline
\end{tabular}

Tabl. XVI - Table de mortalité de la population de Chichery.

\begin{tabular}{|c|c|c|c|}
\hline Âge & $D(x, x+a)$ & $S(x)$ & (a) $q(x)$ \\
\hline 0 & 3 & 15 & 200,0 \\
\hline $1-4$ & 3 & 12 & 250,0 \\
\hline $5-9$ & 1 & 9 & 111,1 \\
\hline $10-14$ & 0 & 8 & 0,0 \\
\hline $15-19$ & 1 & 8 & 125,0 \\
\hline $20-90$ & 7 & 8 & 875,0 \\
\hline Total & 15 & & \\
\hline
\end{tabular}

tion finale de la population immature de Chichery, après ventilation de chaque sujet par classe d'âge, est présentée dans le tableau XV.

La table de mortalité construite à partir de cette répartition se fonde sur l'hypothèse de Halley, admettant que la population étudiée est stable (Sellier, 1996). Le quotient de mortalité entre 0 et 20 ans (20q0) ainsi calculé s'élève à $533 \%$. Compris dans la fourchette attendue pour une espérance de vie à la naissance de 25 ans $(640 \%)$ et 35 ans $(446 \%$ ), ceci vient confirmer une distribution de l'ensemble de la population immature de Chichery conforme au modèle naturel (tabl. XVI).

La représentation graphique des quotients de mortalité de l'échantillon immature et leur comparaison aux quotients théoriques attendus nous permet de constater un recrutement normal des trois premières classes d'âge (fig. 45). Les légères sous-représentations et surreprésentations des [0] et [5-9] ans par rapport aux valeurs médianes du schéma théorique ne sortent néanmoins pas des valeurs maximales. La représentation des adolescents des 10 à 19 ans semble démontrer, au contraire, un recrutement atypique. Cette image doit toutefois être pondérée, compte tenu de l'effectif de ce groupe. Nous avons précédemment souligné l'absence d'individu décédé entre 10 et 14 ans, ce qui explique leur sous-représentation illustrée ici. Si nous partons du principe que nous ne détenons qu'un échantillon de la nécropole, l'interprétation de nos résultats se résume à une alternative.

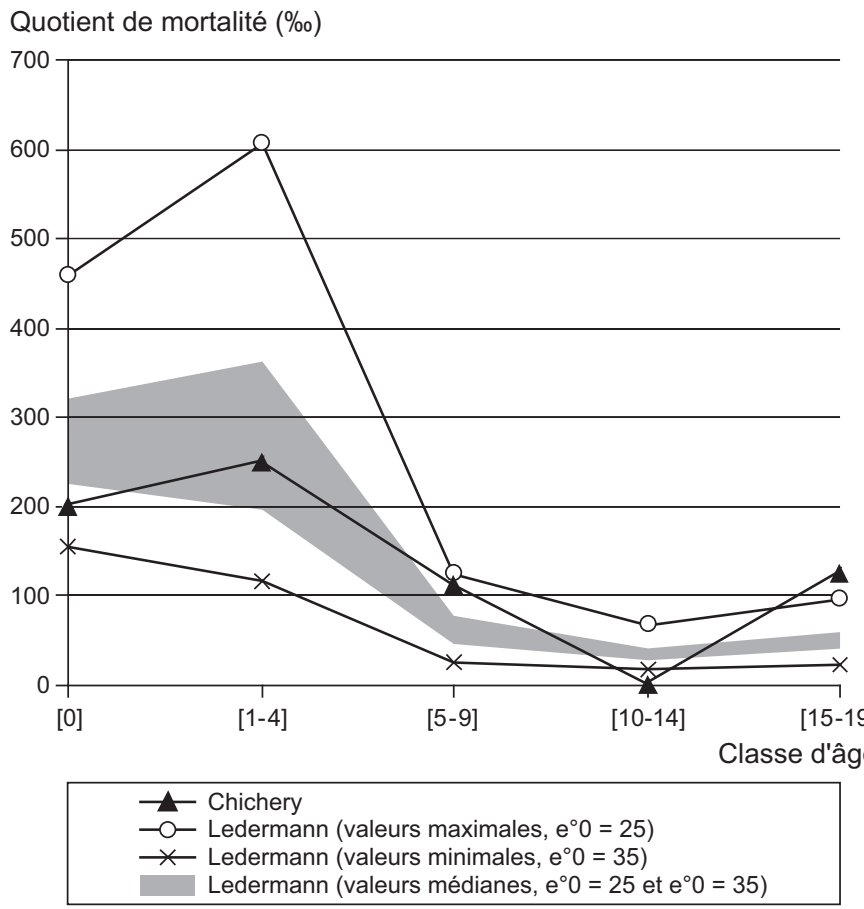

Fig. 45 - Comparaison des quotients de mortalité de la population immature avec les tables de Ledermann (1969).

Hypothèse 1 : les quinze individus constituent un échantillon représentatif de la nécropole (quel que soit le nombre d'individus au total, ils se répartissent dans chaque classe d'âge dans les mêmes proportions que notre échantillon). Dès lors, nous serions en mesure d'affirmer la forte proportion d'individus immatures (décédés avant 20 ans) particulièrement représentés par les premières classes d'âge, conformément à un schéma théorique: constat exceptionnel pour une population de nécropole néolithique. Dans un tel schéma, la sous-représentation des adultes serait à l'origine de l'anomalie observée chez les adolescents.

Hypothèse 2: notre échantillon de quinze individus n'est pas à l'image du recrutement global des inhumés de la nécropole. En d'autres termes, les tombes non fouillées viendraient bouleverser les proportions d'individus observées entre chaque classe d'âge et par là même nuancer, voire infirmer, le constat fait précédemment. Dans cette hypothèse tous les types de recrutement sont envisageables.

En l'état, la composition de la population de Chichery ne traduit aucune sélection des inhumés (les écarts observés ne concernent qu'un individu par classe). Cependant, si nous nous plaçons dans la seconde hypothèse et qu'un nombre important d'adultes se trouve en dehors du secteur fouillé, infirmant une absence de sélection sur l'âge, il 


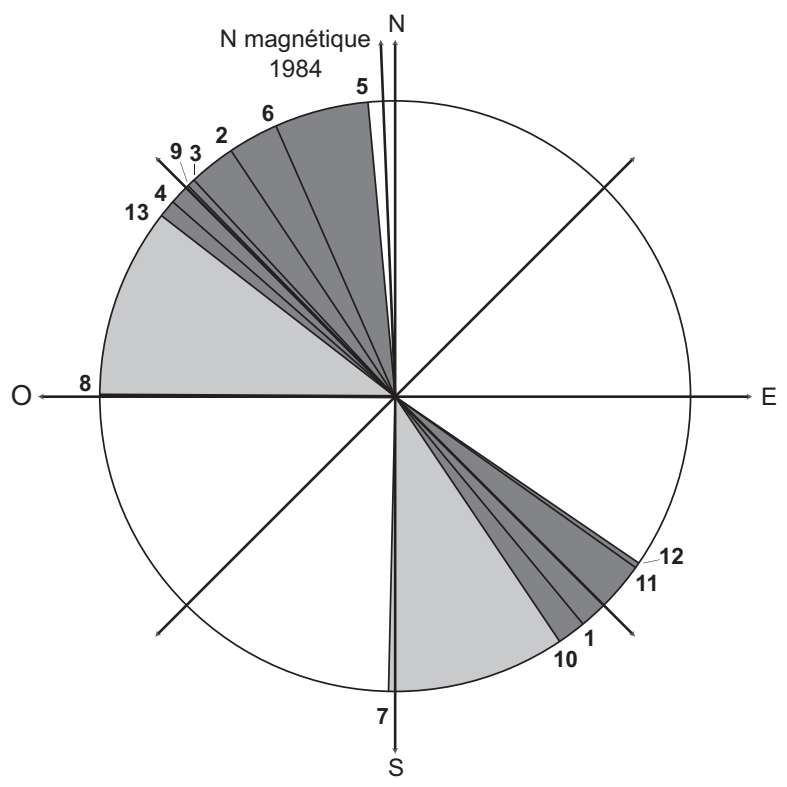

a

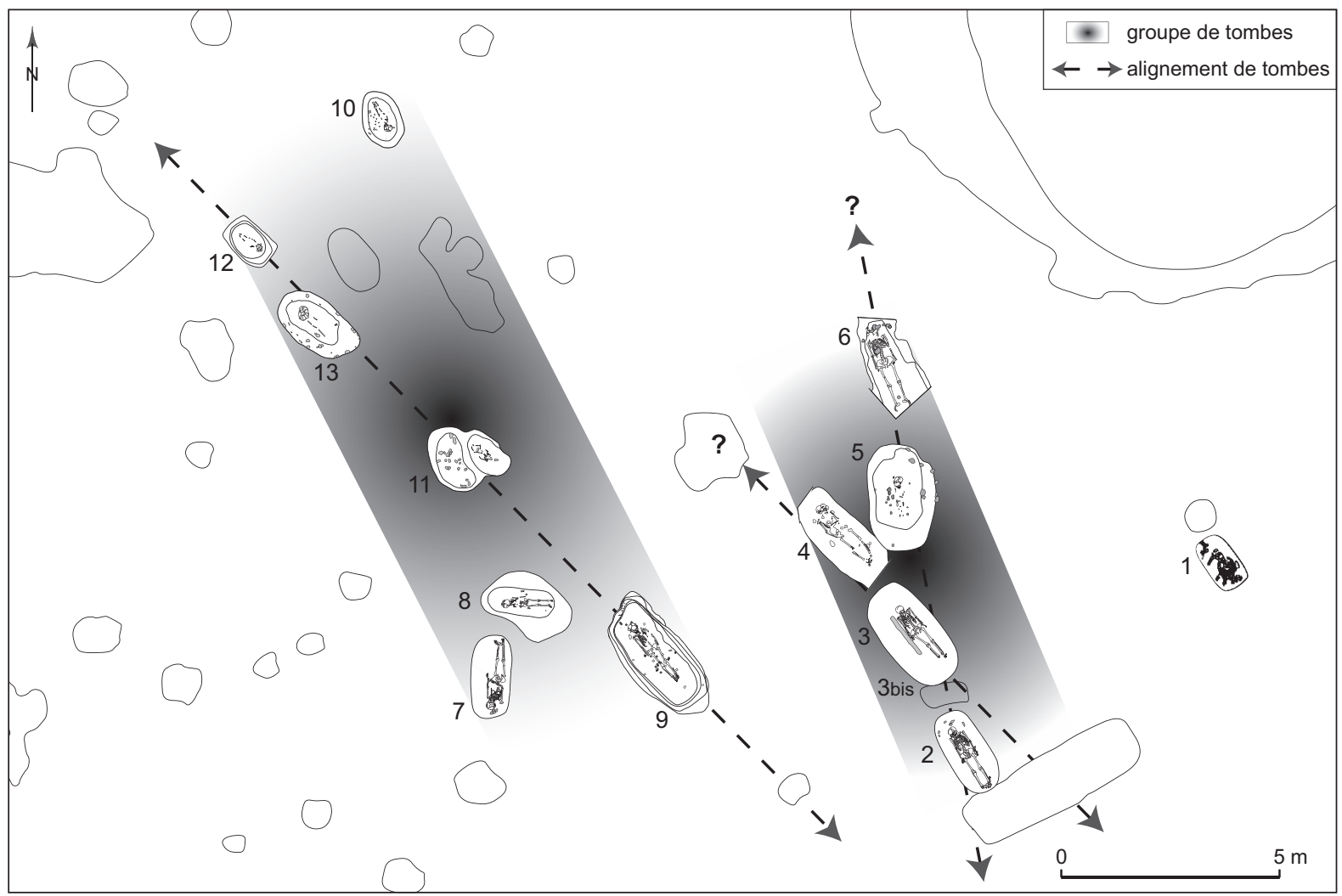

b

Fig. 46 - a, orientation des sépultures. Nous avons corrigé le nord magnétique en fonction de la déclinaison de 1984 (le plan général a été dressé lors de la dernière campagne). L'axe préférentiel est nord-ouest/sud-est, mais sans direction privilégiée pour la tête; $\boldsymbol{b}$, deux (ou trois) ensembles de sépultures; dans le troisième groupe en partant de l'est vers l'ouest, quatre tombes sont parfaitement alignées; dans le deuxième groupe, il semble y avoir deux alignements divergents (DAO: P. Chambon, CNRS). 
faudrait conclure qu'un secteur de la nécropole était plus particulièrement dévolu aux enfants. Le regroupement de la grande majorité des enfants découverts à l'ouest de la zone fouillée suggère d'ores et déjà la volonté de réunir cette partie de la population.

\section{ORGANISATION DE LA NÉGROPOLE}

La variation d'axe des sépultures atteint $90^{\circ}$ entre la sépulture 7, nord-sud, et la sépulture 8, est-ouest. L'axe préférentiel est toutefois nord-ouest/sud-est: neuf sépultures ne s'en écartent pas de plus de $15^{\circ}$ (fig. 46a). Parmi elles, trois ont visiblement une autre référence: nord-sud dans deux cas, est-ouest pour la dernière tombe. L'orientation des corps est au final bien plus variable puisque, pour une même orientation de fosse, on rencontre l'opposition corps dans un sens, corps dans l'autre sens. Si les directions des corps s'opposent, il est en revanche impossible d'évoquer des orientations antipodiques au sens où l'entend C. Jeunesse pour le Rubané (Jeunesse, 1997, p. 63) : il n'existe pas une direction prioritaire avec quelques inversions, cinq ou six sépultures orientées la tête au nord-ouest s'opposent à quatre dont la tête est au sud-est; un sujet a la tête au nord, un autre la tête au sud.

Le problème change de nature à l'examen des tombes concernées. En effet les quatre inhumés orientés la tête au sud-est comprennent les trois plus jeunes sujets et la tombe 1, la plus originale. Les sépultures des cinq adultes en position étendue sur le dos ne présentent que $25^{\circ}$ de variation.

Les sépultures se répartissent spatialement en deux ensembles ou en deux files (fig. 46b). Manifestement, des lignes directrices respectant une orientation nord-ouest/ sud-est apparaissent dans l'implantation des fosses de onze sépultures sur treize. Cette orientation privilégiée est encore discernable dans des alignements de fosses ou de trous de poteau. Chaque ensemble possède sa "personnalité».

La tombe 1 est la seule de son genre sur le gisement; cependant, son orientation obéit à la disposition générale en rangée, suggérant une éventuelle troisième file.

Le second groupe est très dense, les tombes se touchent presque (S2-S6). Trois sépultures respectent l'orientation dominante; elles semblent avoir précédé les deux autres. Tous les sujets ont la tête entre le nord et le nord-ouest. Le groupe comprend une majorité d'adultes. Les deux tombes comportant plusieurs armatures de flèche ainsi que les outils sur bloc sont toutes deux dans cet ensemble. Une troisième sépulture a livré du mobilier.
L'implantation des sept dernières sépultures est plus lâche (tombes 7 à 13). L'alignement de quatre tombes est parfait: l'axe central des tombes 9, 11, 13 et 12 est rigoureusement le même. Six sépultures sur les sept renferment des sujets immatures ( $<20$ ans) et cinq d'entre eux ont moins de 10 ans (fig. 47). Les sépultures dont l'inhumation s'est faite tête au sud appartiennent toutes à ce groupe (sauf la $\mathrm{S} 1$, toujours différente). Bien que numériquement le plus important, aucun dépôt intentionnel d'armes et d'outils en silex n'a été réalisé dans le groupe sud (quoique toutes les sépultures contenaient quelques fragments de silex provenant de la terre de remplissage prélevée probablement à proximité d'une zone d'habitat). En revanche, toutes les sépultures dont le comblement a livré de la céramique sont situées au sud.

La place des adultes, au sein des deux véritables files de sépultures, se trouve prioritairement au sud-est.

La nécropole est structurée autour des sépultures orientées nord-ouest/sud-est. Sur cette base, traduire les différences d'orientation en termes chronologiques paraît raisonnable. Il est plus délicat d'interpréter les inversions d'orientation. Celles-ci concernent des sujets particuliers (notamment les plus jeunes). Quant à la tombe 1, si un décalage chronologique peut expliquer l'inversion, faut-il considérer le maintien de l'axe principal comme fortuit?

La répartition des individus qui présentent une ou plusieurs variations anatomiques non métriques communes nous donne des renseignements supplémentaires sur l'organisation spatiale. C'est le partage de caractères rares au sein d'un sous-ensemble archéologique (dans notre cas, tombes proches ou regroupées) qui est pertinent (Crubézy, Sellier, 1990). Compte tenu du faible effectif de la nécropole, nous avons sélectionné les variations anatomiques présentes chez seulement deux individus et observées sur au moins la moitié du corpus: six caractères crâniens et dentaires, signalés dans les tableaux X et XII (cases grisées). À cette liste nous ajoutons l'inclusion de la canine, variation que nous n'avions pas intégrée a priori dans notre liste, mais que nous avons observée sur deux individus (voir supra, p. 167). Les tests statistiques étant difficiles à appliquer sur ces petits effectifs, il est proposé de faire des projections sur plan des caractères sélectionnés pour appréhender leurs éventuels regroupements (Crubézy et al., 1999). Afin de ne pas tronquer la lecture du plan (fig. 48), nous signalons la présence et l'absence de chacun des sept caractères, de sorte que l'absence de caractère (non observé) ne soit pas confondue avec un caractère non observable (non conservé). 


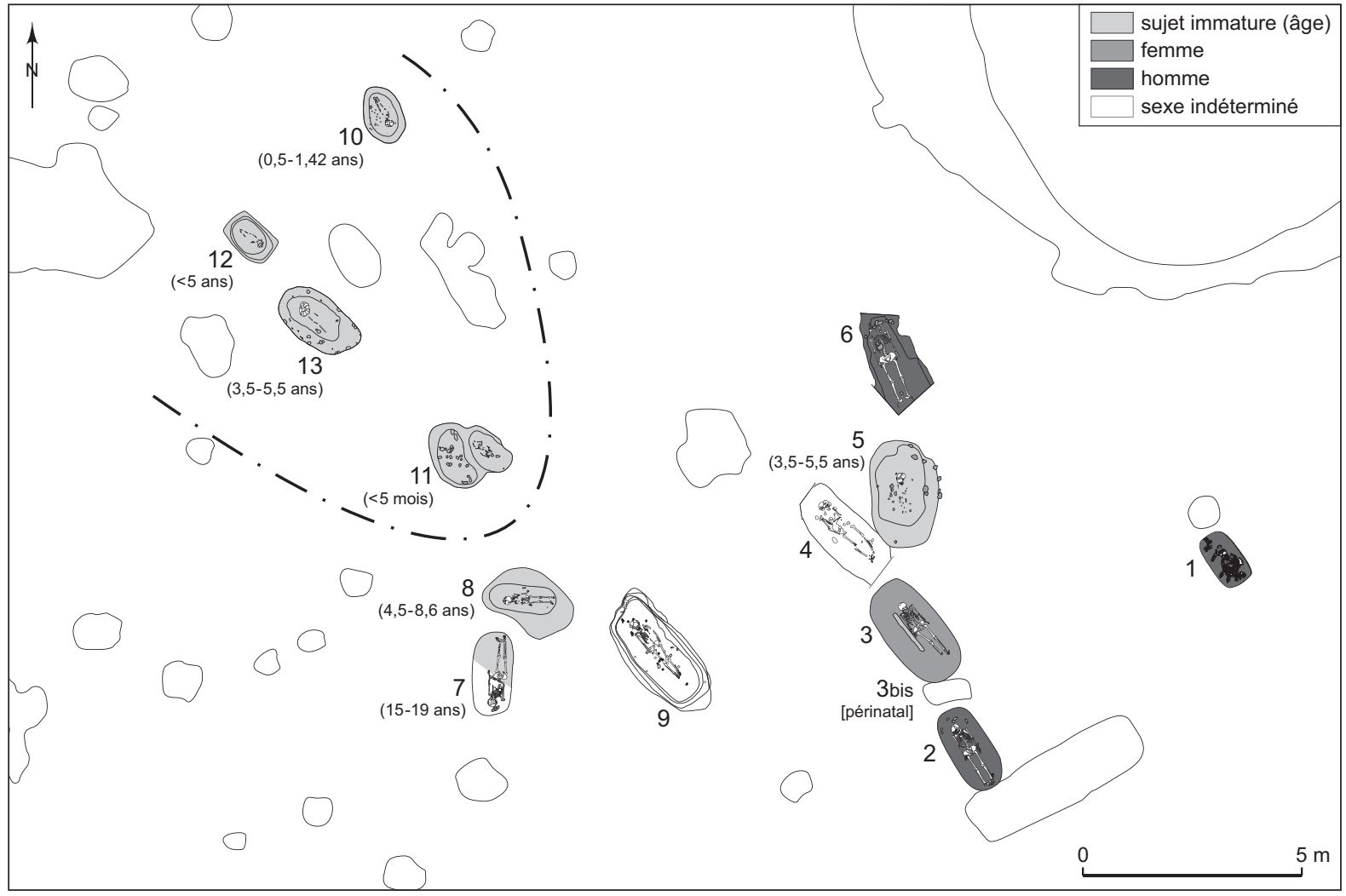

Fig. 47 - Répartition des sujets en fonction de leur âge et de leur sexe. Dans l'hypothèse de trois ensembles, les sujets immatures sont presque tous localisés sur la file sud-est, accompagnés d'un seul adulte. Les plus jeunes sujets sont situés sur l'axe de la tombe 9 (DAO: P. Chambon, CNRS et A. Thomas).

Si on ignore les quatre inhumés pour lesquels l'observation est impossible (5, 11, 12 et 13), seul l'individu 1 ne dispose d'aucun caractère sur les sept sélectionnés dans cette analyse. Quatre variations anatomiques semblent se répartir aléatoirement: le sillon coronoradiculaire de la deuxième incisive supérieure, la présence d'au moins un tubercule mésio-accessoire sur la deuxième molaire supérieure, l'extension de l'émail interradiculaire sur la deuxième molaire inférieure et le torus maxillaire; les individus partageant l'un de ces caractères ne sont clairement pas rapprochés. En revanche, l'homme 4 et la femme 3, les seuls individus à présenter l'inclusion de la canine inférieure, sont inhumés côte à côte. La répartition des caractères crâniens met en évidence un rapprochement entre l'enfant 8 et l'adolescent 7 , seuls individus à présenter une suture incisive. La femme 3 et l'adulte de sexe indéterminé 9 présentent un tubercule marginal de l'os zygomatique. Leurs tombes ne sont pas très éloignées, néanmoins, aucun rapprochement archéologique probant n'est observé entre ces deux individus.

L'interprétation de ces rapprochements reste sujette aux contraintes associées aux caractères non métriques: seule une faible partie du tissu de parenté peut être mise en évidence et, dans le même ordre d'idée, l'absence de regroupement ne traduit pas une absence de lien de parenté (Crubézy, Sellier, 1990).

\section{DATATIONS}

Nous avons procédé à sept datages par la méthode du radiocarbone, réalisés par accélérateur au Centrum voor Isotopen Onderzoek de Groningue. Toutes les mesures sont considérées par le laboratoire comme fiables et comportent un faible écart-type, sauf GrA-33923 pour laquelle la faible quantité de collagène a non seulement imposé un très large écart-type, mais jette également un doute sur la fiabilité de 


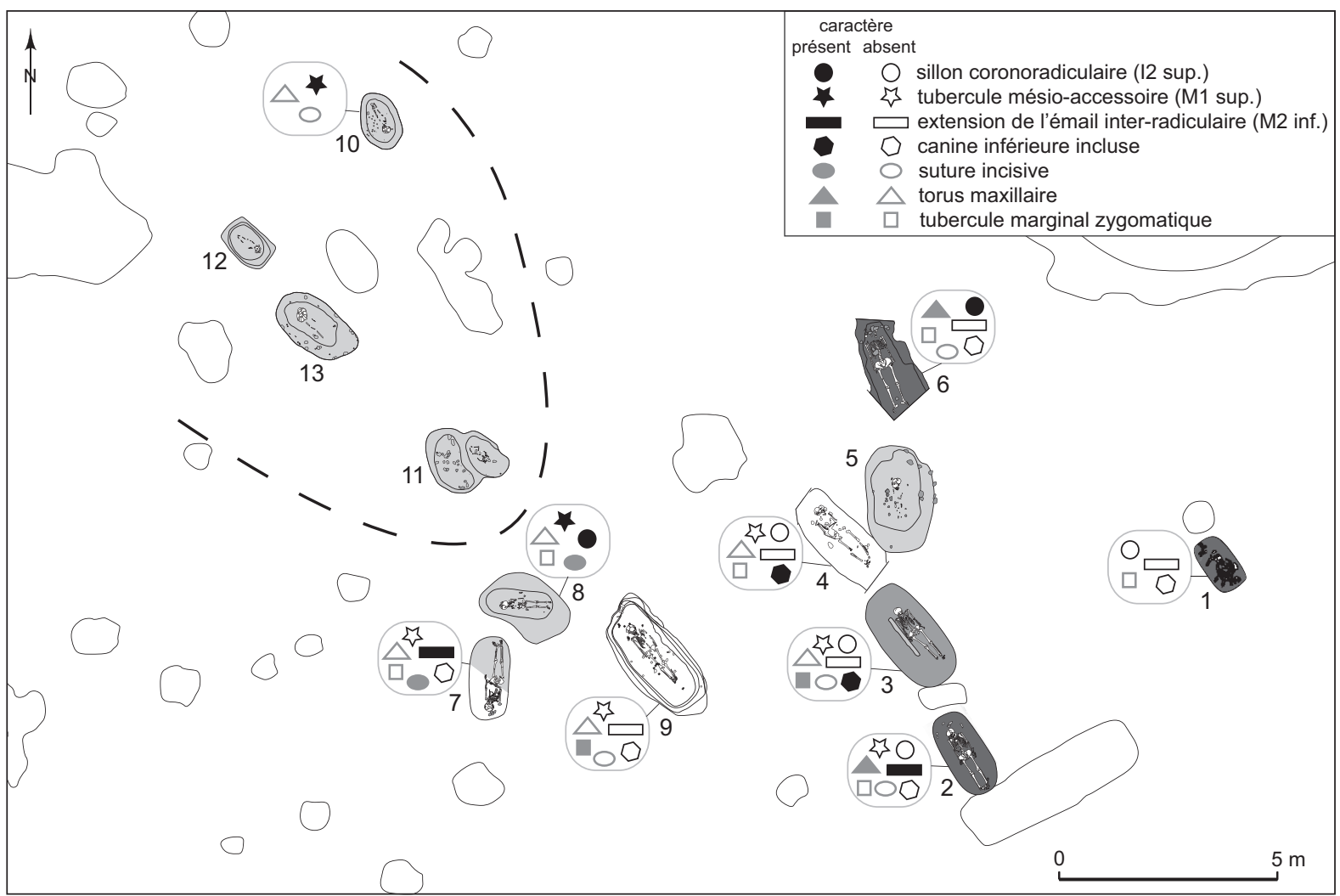

Fig. 48 - Répartition des variations anatomiques non métriques (DAO: A. Thomas).

Tabl. XVII - Datations radiocarbone. La calibration a été réalisée avec le logiciel OxCal 4.0.5.

\begin{tabular}{|c|c|c|c|l|}
\hline Sépulture & Référence & Date BP & Date av. J.-C. & \multicolumn{1}{|c|}{ Échantillon } \\
\hline $\mathbf{1}$ & GrA-40379 & $5385 \pm 30$ & 4335 à 4074 & fragment de crête iliaque \\
\hline $\mathbf{2}$ & GrA-39956 & $5650 \pm 30$ & 4546 à 4373 & phalange proximale de pied \\
\hline $\mathbf{4}$ & GrA 33923 & $6100 \pm 150$ & 5461 à 4686 & esquille proximale fémur droit \\
\hline $\mathbf{6}$ & GrA 33943 & $5690 \pm 40$ & 4681 à 4450 & esquille proximale diaphyse humérus droit \\
\hline $\mathbf{7}$ & GrA 33945 & $5710 \pm 40$ & 4682 à 4459 & fragment de crâne \\
\hline $\mathbf{9}$ & GrA 33946 & $5750 \pm 40$ & 4703 à 4500 & fragment de crâne \\
\hline $\mathbf{1 0}$ & GrA 33948 & $5745 \pm 40$ & 4695 à 4496 & esquille proximale fémur droit \\
\hline
\end{tabular}

la mesure (tabl. XVII). Le support utilisé a, dans tous les cas, été le fragment d'un os identifié sur les photographies comme appartenant bien au sujet de la sépulture correspondante.

Six échantillons proviennent des sépultures en position étendue sur le dos, le dernier à l'unique tombe renvoyant sans hésitation à un dispositif différent (sépulture 1). Les résultats offrent une grande cohérence: seule la sépulture 1 sort une nouvelle fois du lot, confirmant définitivement sa distance avec le reste de la nécropole. Hormis cette tombe et la sépulture 4, dont nous préférons éviter d'utiliser la date, l'intervalle de recouvrement des datations ne dépasse pas 50 ans entre 4550 et 4500 av. J.-C. En revanche la somme des intervalles donne un intervalle de moins de 400 ans, entre 4745 et 4300 av. J.-C. Nous ne pouvons, sur cette base, proposer une chronologie interne de la nécropole (fig. 49).

La somme des intervalles couvre une période trop grande pour la nécropole. La cohérence de l'implantation des tombes, l'absence de recoupement et l'homogénéité présumée des dispositifs sépulcraux plaident pour une 


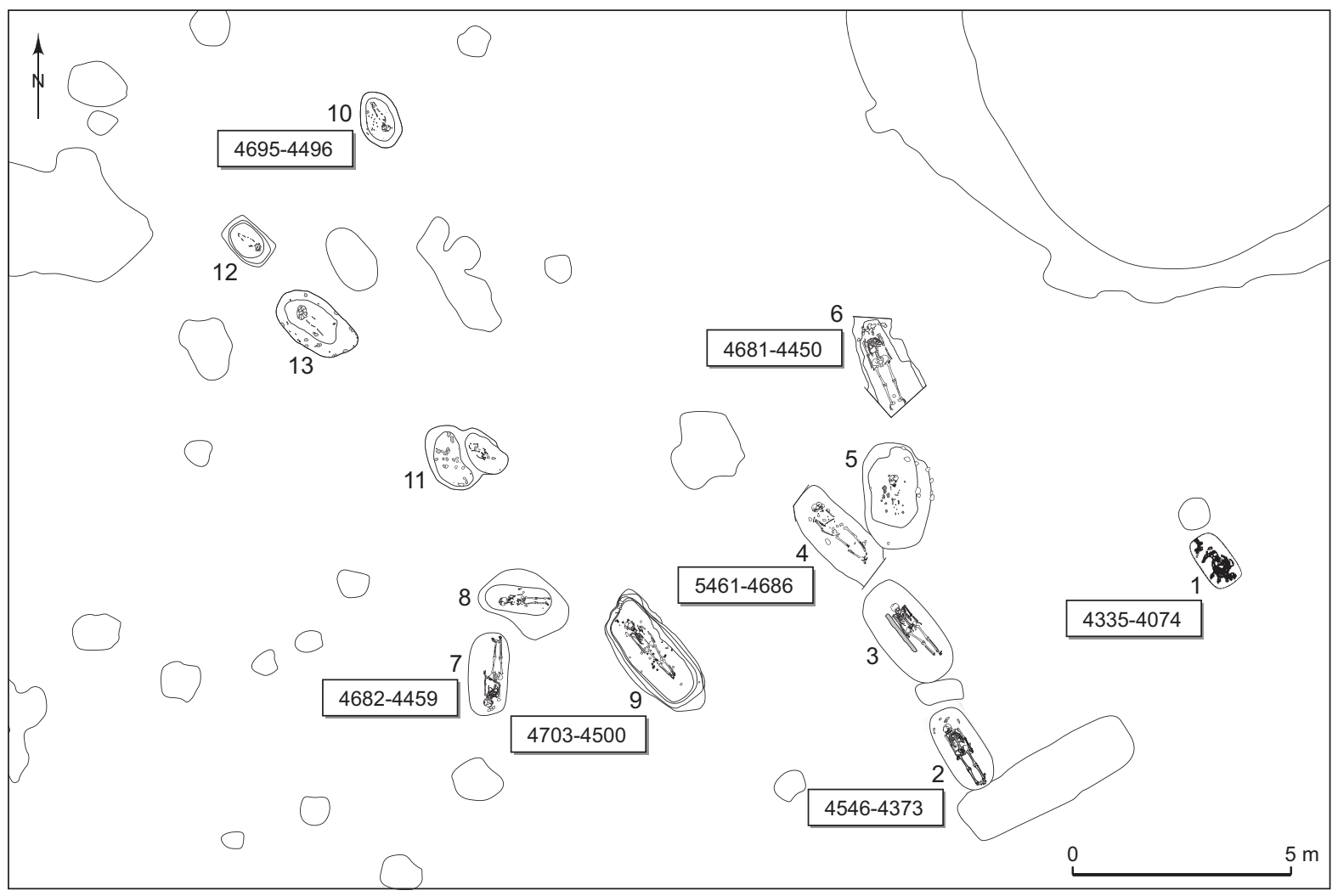

Fig. 49 - Dates des sépultures et organisation de la nécropole; il n'y a pas d'écart significatif entre les deux files de sépultures. Seule la tombe 1 apparaît indiscutablement récente (DAO: P. Chambon, CNRS).

durée assez courte, inférieure aux quatre siècles figurés par la somme des intervalles. Inversement, il n'y a sans doute pas lieu de considérer que toutes les tombes sont contemporaines: l'intervalle de recouvrement des dates est sans doute trop court.

La nécropole peut débuter avant 4600 av. J.-C., elle ne se prolonge sans doute pas, hormis la tombe 1 , après 4450 av. J.-C. Elle se développe donc - pour la partie connue - avant le milieu du $\mathrm{V}^{\mathrm{e}}$ millénaire (fig. 50). En ce qui concerne les sépultures contenant un sujet en position étendue sur le dos (type Balloy), il s'agit, au sein du maigre corpus de dates actuellement disponibles, du lot de tombes le plus ancien. Seules quelques mesures ont actuellement des intervalles débutant avant 4500 av. J.-C. Si on considère l'ensemble des sépultures traditionnellement associées à la culture de Cerny, c'est-à-dire, outre le type Balloy, ce que nous avons nommé, sans pouvoir le définir précisément, le type Orville (Chambon et al., 2009), les intervalles obtenus pour Chichery paraissent à nouveau très anciens.

\section{CONTEXTE CHRONOLOGIQUE ET CULTUREL}

Douze sépultures de Sur les Pâtureaux se rattachent au type Balloy (ou Nm-E1) (Chambon, Lanchon, 2003; Chambon et al., 2009), c'est-à-dire aux sépultures majoritaires en Bassin parisien au Néolithique moyen I: plus d'une centaine d'exemples sont désormais connus. Ces tombes correspondent à l'inhumation en contenant rigide mobile. La position étendue sur le dos n'est qu'un corollaire du contenant mobile. La double structure, cercueil et caveau, est ainsi attestée à la Noue Fenard et à la Porte aux Bergers (Vignely, Seine-et-Marne), aux Sablons (Gron, Yonne) et à Macherin (Monéteau, Yonne) (Augereau, Chambon dir., sous presse) en plus de Balloy et de Passy. Les difficultés qui président à son identification suggèrent que, dans de nombreux cas, nous échouons à mettre en évidence un tel dispositif. En l'absence des critères décisifs, rappelons que de telles structures impliquent que le squelette soit étendu sur le dos au centre d'une fosse surdimensionnée (par rap- 

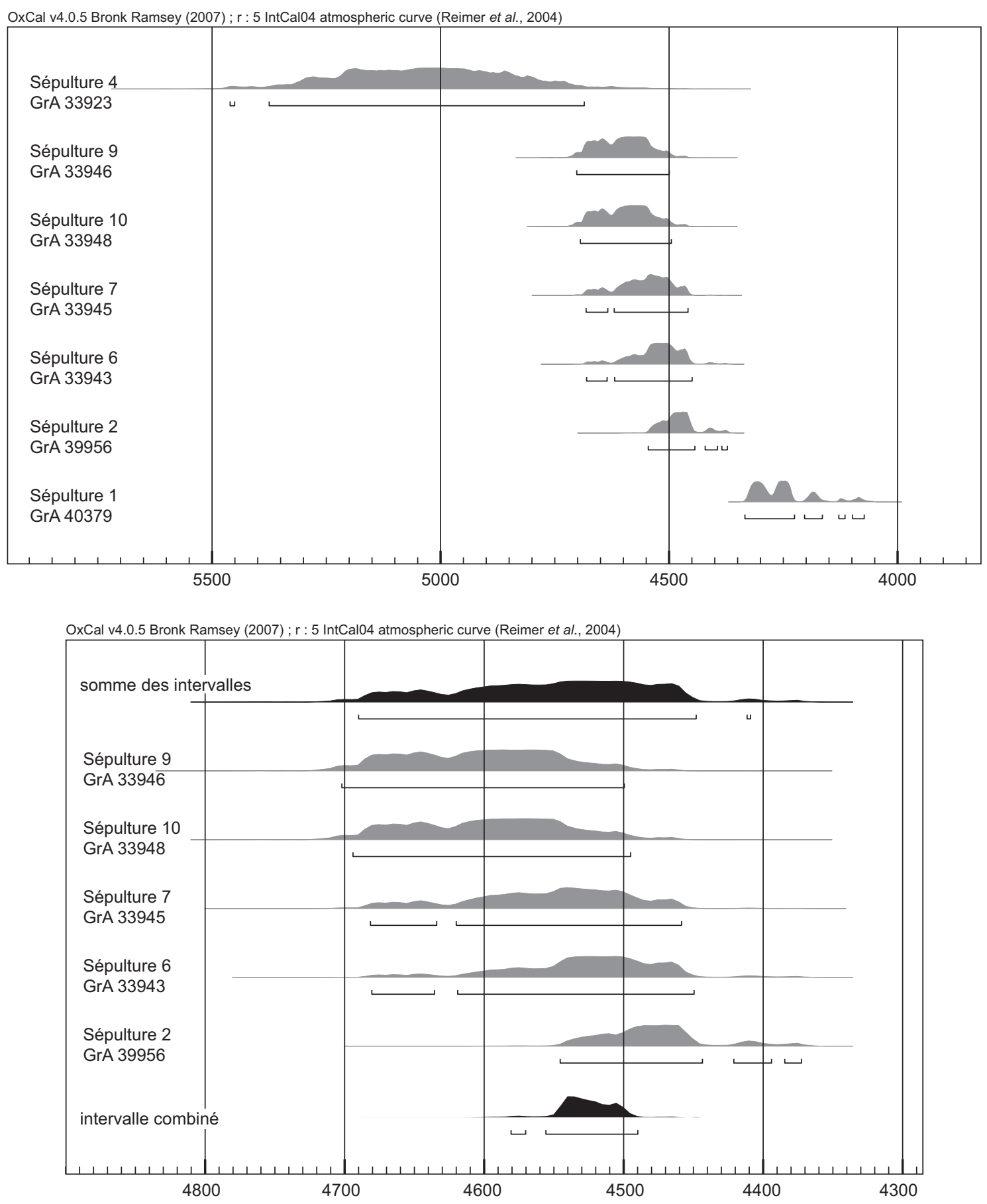

Fig. 50 - Histogrammes de calibration des dates de la nécropole (les courbes ont été réalisées à partir du logiciel libre OxCal 4.0.5 @ Christopher Bronk Ramsey, 2007): en haut, les histogrammes pour les sept mesures réalisées; en bas, les cinq dates les plus cohérentes, encadrées par l'histogramme correspondant à la somme des cinq dates et par l'histogramme de l'intervalle commun aux cinq mesures (DAO: P. Chambon, CNRS).

port à l'espace effectivement occupé par le squelette), fosse dont le profil évoque une baignoire.

Le type Balloy, dans le Bassin parisien, correspond à l'horizon Cerny. Il est traditionnellement associé à la culture même de Cerny, bien que les éléments propres à cette culture soient peu présents dans les tombes. Il s'agit en fait d'un parallèle entre ces sépultures et cette culture, dont les datations et la localisation géographique concordent 


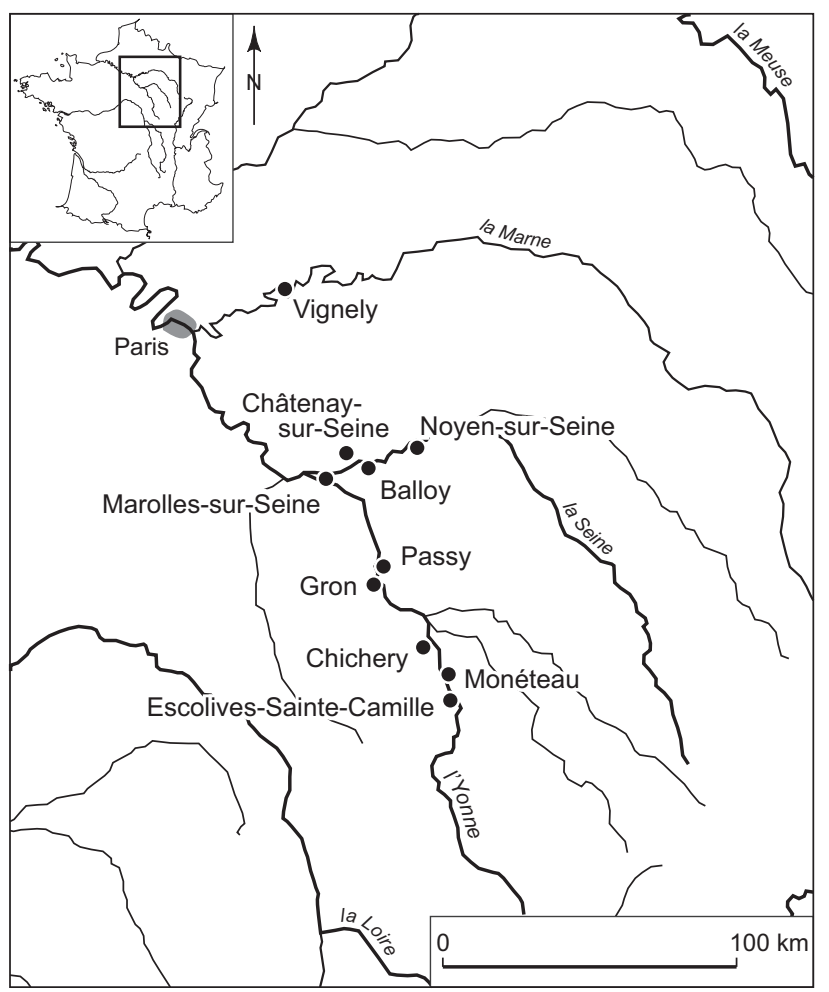

Fig. 51 - Sites ayant livré des sépultures type Balloy (Nm-E, in Chambon, Lanchon, 2003) en Bassin parisien: la Porte aux Bergers et la Noue Fenard à Vignely; le Haut des Nachères à Noyen-sur-Seine; les Réaudins à Balloy; les Pâtures à Châtenay-sur-Seine; les Gours aux Lions à Marolles-sur-Seine; la Sablonnière et Richebourg à Passy; les Sablons à Gron; Sur les Pâtureaux à Chichery; Macherin à Monéteau; la Pièce de l'Étang à Escolives-Sainte-Camille (DAO: P. Chambon, CNRS et P. Pihuit, Inrap).

globalement. La céramique, retrouvée épisodiquement, comprend bien quelques formes et décors Cerny mais aussi des exemplaires atypiques, voire exogènes. Le mobilier lithique est le matériel le plus abondant, et c'est généralement lui qui assure l'attribution culturelle. Dans des zones où l'impact Cerny est faible, on peut s'interroger sur la valeur d'un pic-ciseau ou d'une armature tranchante pour justifier cette appellation. En outre, ce type de sépulture n'est pour l'instant pas attesté dans toute l'aire d'extension de la culture de Cerny (fig. 51) : même dans des vallées aussi explorées que celles de l'Oise ou de l'Aisne, les sépultures restent absentes ou exceptionnelles (Chambon et al., 2009).

Si la démonstration reste à faire que des sépultures type Balloy en Bassin parisien peuvent se rattacher à une autre culture que le Cerny, dans la vallée du Rhin c'est tout le Mittelneolithikum qui est concerné par ce type de tombe. Le cycle débute avec le Hinkelstein en Alsace, deux à trois siècles avant le Cerny et se termine avec le Rössen dont l'extrême fin est mise en parallèle avec lui. Les sépultures type Balloy pourraient traduire l'arrivée d'influx orientaux durant la dernière étape du Mittelneolithikum. En effet, l'impact de ces cultures rhénanes dans le cour du Bassin parisien est supposé faible jusqu'au Rössen III ou Post-Rössen. La situation est cependant différente en Bourgogne où le Cerny demeure une entité mal représentée. La nécropole monumentale d'Escolives-Sainte-Camille, partiellement fouillée, n'a livré que trois formes céramiques, dont deux ne s'inscrivent pas dans le registre Cerny (Duhamel, 2004 et 2006) ; la présence de monuments funéraires allongés constitue-t-elle un argument suffisant pour conclure que la nécropole se range bien dans l'ensemble Cerny? En outre, dans la vallée de l'Yonne, en amont de Joigny, la culture de Cerny n'est connue qu'à travers le matériel de quelques gisements qui ne livrent pas la totalité des éléments caractéristiques de cette culture. J.-P. Thévenot et, après lui, M. Prestreau ont insisté sur l'ancienneté des influx Rössen en Bourgogne, au-delà du Morvan dans la plaine de la Saône, mais aussi en deçà, dans l'Yonne (Thévenot dir., 2005; Prestreau, 2006). Le mobilier associé aux sépultures de Sur les Pâtureaux ne renvoie pas à la vallée du Rhin, si on excepte la curieuse armature à base concave dont l'attribution au groupe des perçantes reste problématique. Le lien réside davantage dans l'ancienneté des datations radiocarbone et dans l'orientation des sépultures.

L'hypothèse d'une attribution au Cerny de la nécropole de Sur les Pâtureaux n'est réellement étayée que par le matériel lithique associé aux sépultures et par l'absence d'un autre mobilier culturellement significatif. La confrontation avec les autres sépultures attribuées à cette culture, notamment les sépultures type Balloy, souligne par contraste l'absence de la parure et celle de l'industrie osseuse, deux types de mobilier présents dans toutes les nécropoles Cerny. L’orientation des sépultures évoque davantage les nécropoles du Mittelneolithikum (fig. 52) que les ensembles Cerny: l'axe nord-ouest/sud-est prévaut aussi bien à Jechtingen en Bade-Wurtemberg (Kasa, 2002) qu'à Trebur en Hesse (Spatz, 1999), Lingolsheim (Forrer, 1911 et 1938) ou Rosheim (Boës et al., 2000), les deux derniers dans le Bas-Rhin; la direction de la tête s'inverse vraisemblablement d'une culture à l'autre mais l'axe demeure. Enfin, la datation ancienne de la nécropole bat une nouvelle fois en brèche le schéma simple d'une étape ancienne du Cerny caractérisée par le faciès céramique éponyme (ou Orville), associée aux sépultures à inhumation fléchie (Louboutin, Simonin, 1997), suivie par une étape récente comportant le faciès céramique Barbuise, associée aux nécropoles monumentales et aux tombes de type Balloy. Quelle que soit la 

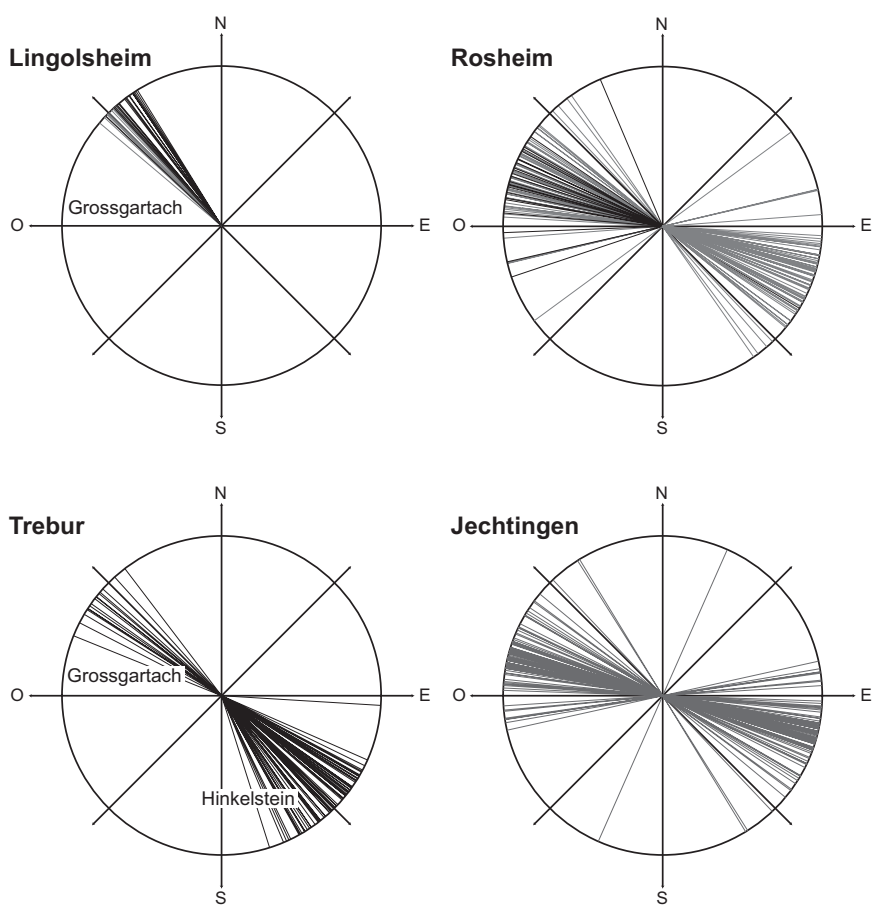

Fig. 52 - Orientation des tombes dans les principales nécropoles du Mittelneolithikum. À Lingolsheim l'orientation reste approximative, singulièrement pour les tombes publiées en 1938, figurées en gris sur le diagramme (Forrer, 1911, fig. 173 et 1938, pl. 39); à Rosheim, nous ne connaissons la direction de la tête que pour une minorité de sépultures (en noir); pour les autres c'est l'axe global qui est indiqué en gris (d'après Boës et al., 2000); dans le cas de Jechtingen, c'est encore l'axe global qui est figuré en gris (d’après Kasa, 2002); Trebur, d'après Spatz, 1999 (DAO: P. Chambon, CNRS).

valeur accordée aux faciès céramiques, les sépultures type Balloy existent dès le début de l'horizon Cerny.

Le lien entre Sur les Pâtureaux et le Chasséen est plus ténu. L'une des armatures de la sépulture 2 évoque directement cette culture, ce qui a pu conduire M. Prestreau à la considérer comme telle (Prestreau, 2006). Les datations radiocarbone de la nécropole, dans lesquelles s'insère parfaitement celle de la sépulture 2, sont une difficulté. En effet, si on ne peut désormais plus nier une présence chasséenne entre 4400 et 4300 av. J.-C. dans l'Yonne, il faudrait ici considérer que le premier impact de cette entité intervient une centaine d'années avant ! La nécropole de Sur les Pâtureaux ne constitue ici qu'une épine supplémentaire dans le problème du Chasséen de Bourgogne.

L'inclusion de la sépulture 1 dans la nécropole de Sur les Pâtureaux pose plus de questions qu'elle n'en résout. La datation récente ne permet pas d'y voir, comme à Monéteau (Chambon et al., 2006), la coexistence de dispositifs sépulcraux et plus globalement de pratiques funéraires différentes. L'intervalle est trop récent pour l'horizon Cerny et ne permet donc pas d'associer cette tombe au type Orville (Chambon et al., 2009). En revanche, elle s'intègre facilement dans le contexte local des sépultures où l'inhumé repose selon une orientation sud-est/nordouest, en position fléchie, les membres inférieurs sur le côté gauche. La pauvreté ou l'absence de mobilier est un trait récurrent de ces sépultures à l'Étang David, sur la même commune (Pellet, 1978), comme dans les nécropoles des Noisats, à Gurgy (Rottier, 2007), ou de Macherin, à Monéteau (Augereau, Chambon dir., sous presse). La localisation de la tombe suggère une forme de continuité de la nécropole, peut-être également une position dans le haut de l'intervalle fournit par la calibration. Elle milite encore pour le prolongement du gisement hors du secteur exploré.

La mise au jour de restes osseux incinérés ajoute un cas supplémentaire au sein du Néolithique moyen I de la Bassée et de la vallée de l'Yonne, après les trouvailles de Balloy (Mordant, 1997), de Passy (Duhamel et al., 1997), Gron (Müller et al., 1997) et plus récemment de Monéteau (Augereau, Chambon dir., sous presse). L'absence d'une structure d'accueil spécifique semble la norme pour ces dépôts. Toutefois, leur présence presque systématique dans les ensembles funéraires de cette période ne peut plus être qualifiée d'anecdotique. Cependant, leur petit nombre et les traces fugaces que ces dépôts laissent n'autorisent pas encore une véritable interprétation.

Le recrutement accentue les tendances observées dans les nécropoles du Néolithique moyen I. Les sujets immatures sont nombreux, à l'image des nécropoles de Balloy, mais on rencontre ici de très jeunes sujets en position centrale dans les sépultures. Le recrutement de la nécropole est compatible avec une démographie naturelle, ce qui ne se rencontre, pour une période comparable, qu'à Rosheim (Boës et al., 2000). Contrairement à Rosheim et sa centaine d'inhumés, la fouille vraisemblablement partielle de la nécropole de Sur les Pâtureaux ne permet pas d'assurer que cette représentation équilibrée n'est pas fortuite. La répartition des sujets semble tenir compte de l'âge des inhumés, en revanche le sexe n'influence pas (visiblement) la distribution des adultes: femmes et hommes se côtoient, ce qui n'est pas envisageable dans les nécropoles de Balloy ou de Gron, par exemple (Chambon, 1997 et 2003). Inversement, les femmes et les hommes sont mélangés dans les nécropoles du Mittelneolithikum rhénan et les coffres de Suisse occidentale ou à Monéteau, situé à quelques kilomètres au sud.

Le mobilier s'inscrit dans l'éventail des objets habituellement rencontrés dans les tombes type Balloy. Sa relative 
indigence aussi. Le mobilier le plus original est constitué par les deux pics-ciseaux des sépultures 2 et 5 . L'absence totale d'industrie osseuse est plus rare, ce trait n'est partagé qu'avec les quatre sépultures de la Noue Fenard à Vignely.

L'implantation des sépultures constitue le point le plus original de la petite nécropole de Sur les Pâtureaux. Les files de tombes trouvent un écho dans les alignements observés sur l'axe central de certains monuments allongés: le plus important est l'ensemble I-II des Réaudins à Balloy, avec neuf tombes; le monument $\mathrm{E}$ de la Pièce de l'Étang, à Escolives-Sainte-Camille, comportait cinq sépultures et le monument 17 de Passy en a livré quatre. Ces cas restent rares, les enclos allongés sont le plus souvent dédiés à une ou deux tombes seulement, quelle que soit leur taille (Chambon, 2003). Toutefois, la comparaison avec les nécropoles monumentales s'arrête là. Si la tentation fut parfois grande d'inscrire, a posteriori, les sépultures de Sur les Pâtureaux au sein de monuments du type Passy, la topographie permet d'exclure totalement cette hypothèse: les deux (ou trois) rangées de sépultures sont bien trop rapprochées pour qu'on puisse envisager de faire passer un fossé entre elles; la largeur des monuments est le trait le plus constant, autour de 7-8 m. Enfin, la ventilation des sépultures, dans les monuments, interdit d'y mélanger femmes et hommes.

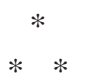

La nécropole de Sur les Pâtureaux à Chichery n'est sans doute pas connue dans son intégralité. Seuls $600 \mathrm{~m}^{2}$ ont été explorés et, tant au sud-est qu'au nord-ouest de cette zone, la distance entre la limite du décapage et les sépultures les plus proches ne dépasse pas 3,50 m. Les treize tombes connues à ce jour ne sauraient constituer qu'un nombre très minimal. On peut envisager que la nécropole est implantée sur un dôme de gravier, entre deux paléochenaux; cependant la surface décapée n'en a atteint aucun et rien ne nous autorise à considérer que la nécropole se développe dans une direction plutôt que dans une autre.

Les sépultures mises au jour se rapportent toutes, sauf une, au type Balloy. Ces sépultures sont habituellement associées, en Bassin parisien, aux nécropoles monumentales de type Passy. Ce n'est pas le cas à Sur les Pâtureaux, où il n'est pas possible d'inscrire les tombes dans de tels monuments. L'extension de la fouille interdit, en revanche, d'exclure l'hypothèse de dispositifs monumentaux d'une autre forme.

$\mathrm{Si}$ on peut vraisemblablement considérer toutes les sépultures comme individuelles, la population s'élève cependant à quinze sujets. L'une d'elles comprenait en effet un sujet décédé en période périnatale, peut-être in utero, et le comblement d'une autre tombe a livré les restes d'une incinération. La proportion de sujets immatures et singulièrement de sujets de moins de 5 ans est conforme à une démographie naturelle. Les sépultures sont implantées sur deux (ou trois) lignes parallèles axées nord-ouest/sud-est, comme la majorité des sépultures, et chaque ligne comprend de une à six tombes. Les sujets les plus jeunes sont regroupés sur l'une d'entre elles. En revanche, femmes et hommes sont associés.

Le mobilier est assez indigent. L'association avec la sépulture n'est probante que dans cinq cas et il s'agit à chaque fois d'industrie lithique. On retiendra l'analogie des dépôts dans les sépultures 2 et 5 , ainsi que l'aspect miniature de l'outil sur bloc, dans la tombe d'enfant, comparé à celui qui accompagne l'adulte. Pour ce dernier sujet l'échantillonnage varié d'armatures ne peut apparaître comme fortuit. Les quelques objets supplémentaires en silex sont des lames ou des fragments de lames qui apparaissent régulièrement au niveau des épaules. Étaient-ils accrochés au vêtement ou placés dans une poche?

Aucune trace d'usage n'a pu être identifiée sur ce matériel: si leur état n'autorise pas une réelle étude tracéologique, les pièces ne portent aucun stigmate d'utilisation prolongée. La céramique se réduit à des tessons épars dont la présence dans le comblement des sépultures est vraisemblablement accidentelle.

Les sépultures type Balloy sont conventionnellement rapportées au Cerny dans le Bassin parisien, ce que semble confirmer l'industrie lithique du gisement et ce que ne contredisent pas les tessons mis au jour dans le remplissage de quelques tombes. Cependant, trois caractères distinguent la nécropole. L'orientation des sépultures selon un axe sud-est/nord-ouest se retrouve dans les nécropoles rhénanes, mais dans le Bassin parisien l'axe est-ouest est plébiscité. Le mobilier des tombes comprend bien les artefacts en silex habituel en contexte Cerny, mais la parure et l'industrie osseuse qui les accompagnent ailleurs font ici totalement défaut. Enfin les dates radiocarbone sont antérieures de deux siècles à celles actuellement disponibles pour les tombes Cerny.

La nécropole de Sur les Pâtureaux montre des influences anciennes du Mittelneolithikum dans le sud-est du Bassin parisien; elle témoignerait du rôle joué par les cultures rhénanes dans le développement de la tradition funéraire liée aux sépultures de type Balloy. 


\title{
BIBLIOGRAPHIE
}

\author{
ABRÉViations \\ ADRAHP Association pour le développement de la recherche archéologique et historique en Périgord. \\ AJPA American Journal of Physical Anthropology. \\ APRAA Association pour la promotion de la recherche archéologique en Alsace. \\ APRAIF Association pour la promotion de la recherche en Île-de-France. \\ BMSAP Bulletins et mémoires de la Société d'anthropologie de Paris. \\ BSPF Bulletin de la Société préhistorique française. \\ DAF Documents d'archéologie française. \\ DRAC Direction régionale des Affaires culturelles. \\ MMPIF Mémoires du musée de Préhistoire d'île-de-France. \\ MSH Maison des sciences de l'homme. \\ RAE Revue archéologique de l'Est. \\ SPF Société préhistorique française. \\ SRA Service régional de l'archéologie.
}

\section{AUgereau A.}

2004: L'Industrie du silex du $V^{e}$ au IV $V^{e}$ millénaire dans le sud-est du Bassin parisien: Rubané, Villeneuve-Saint-Germain et groupe de Noyen, Paris, MSH (coll. DAF, 97), $220 \mathrm{p}$.

2008: «L'industrie lithique de Gurgy, les Noisats (Yonne): campagnes 20042006 », in CHAMbON P. (DIR.), Évolution, coexistence et confrontation de pratiques funéraires entre 4500 et 4000 av. J.-C. sur un microterritoire: la vallée de l'Yonne entre le Serein et le Baulche, Rapport de PCR, SRA de Bourgogne, p. 74-84.

Augereau A., Bostyn F.

1997: «Évolution de l'industrie du silex entre les groupes de Villeneuve-SaintGermain et de Cerny dans la France du Nord», in CONSTANTIN C., MORDANT D., Simonin D. (DIR.), La Culture de Cerny: nouvelle économie, nouvelle société au Néolithique, Actes du colloque international de Nemours, 1994, Nemours, APRAIF (coll. MMPIF, 6), p. 25-38.

Augereau A., Brunet P., Costa L., CotTIAUX R., HAMON T., IHUEL E., LANGRY-FRANCOIS F., MAGNE P., Maingaud A., Mallet N., Martineau R., MiLle B., Millet-RichaRd L.-A., POLlONi A., RENARD C., RichaRd G., SAlanova L., SAmzun A., Sidéra I., SOHN M.

2007: «Le Néolithique récent dans le centre-nord de la France (3400330/2800-2700 av. J.-C.) : l'avenir du Seine-Oise-Marne en question", in Un siècle de construction du discours scientifique en Préhistoire, Congrès préhistorique de
France, 24e session, Avignon, 2004, Paris, SPF, p. 165-184.

Augereau A., Chambon P. (Dir.)

Sous presse: Les Occupations néolithiques de Monéteau «Macherin» (Yonne), Paris, SPF (coll. Mémoires de la SPF).

Augereau A., Chambon P. avec la collab. de SIDÉRA I.

2003: "Nature et statut des mobiliers funéraires de la nécropole chasséenne de Monéteau (Yonne)", in CHAmbON P., LECLERC J. (DIR.), Les Pratiques funéraires néolithiques avant 3500 av. J.-C. en France et dans les régions limitrophes, Actes de la table ronde SPF, Saint-Germain-en-Laye, juin 2001, Paris, SPF (coll. Mémoires de la SPF, 33), p. 131-145.

2005: «Les occupations néolithiques de Monéteau, "Sur Macherin" (Yonne) : données préliminaires», $R A E, 54$, p. 51-70.

BINDER D.

1991: «Facteurs de variabilité des outillages lithiques chasséens dans le sud-est de la France", in BEECHING A. ET AL. (DIR.), Identité du Chasséen, Actes du colloque international de Nemours, 1989, Nemours, APRAIF (coll. MMPIF, 4), p. 261-272.

\section{BIRKNER R.}

1980: L'Image radiologique typique du squelette, Paris, Maloine, 564 p.

Boës E., Jeunesse C., Alix G., Broqua C. 2000: «La nécropole Néolithique moyen de Rosheim "Mittelfeld" (Bas-Rhin): premiers résultats concernant le recrute- ment des individus", in Internéo 3, Actes de la journée d'information du 2 déc. 2000, Paris, SPF, p. 37-49.

BRÄUER G.

1988: «Osteometrie», in KNUSSMANN R., SCHWIDETZKY I., JÜRGENS H., Zieglemayer H. (DIR.), Anthropologie, Handbuch der vergleichenden Biologie des Menschen, 4. Auflage des Lehrbuchs des Anthropologie begründet von R. Martin -I/1Wesen und Methoden des Anthropologie, Stuttgart, Gustav Fischer, p. 160-223.

BROTHWELL D. R.

1965: Digging up Bones: the Excavation, Treatment and Study of Human Skeletal Remains, Londres, British Museum (coll. Natural History), 194 p.

BRUZEK J.

2002: «A Method for Visual Determination of Sex, Using the Human Hip Bone», AJPA, 117, p. 157-168.

\section{Chambon P.}

1997: «La nécropole de Balloy "Les Réaudins": approche archéo-anthropologique", in CONSTANTIN C., MORDANT D., SIMONIN D. (DIR.), La Culture de Cerny: nouvelle économie, nouvelle société au Néolithique, Actes du colloque international de Nemours, 1994, Nemours, APRAIF (coll. MMPIF, 6), p. 489-498

2003: «Revoir Passy à la lumière de Balloy: les nécropoles monumentales Cerny $\mathrm{du}$ bassin Seine-Yonne», BSPF, 100, 3, p. 505-515. 
Chambon P., Augereau A., BONNARdin S., MEUNIER K., THIOL S. avec la collab. de SIDÉRA I., TRISTAN C. 2006: «Une nécropole chasséenne à Monéteau (Yonne) », in FOUERRÉ P., Chevillot C., Courtaud P., Ferrullo O., Leroyer C. (DIR.), Paysages et peuplements, aspects culturels et chronologiques en France méridionale: actualité de la recherche, Actes des VIe rencontres méridionales de Préhistoire récente, Périgueux, 14-16 oct. 2004, Périgueux, ADRAHP (coll. Suppl. à Préhistoire du Sud-Ouest, $11)$, p. 143-162.

CHAMBON P., LANCHON Y.

2003: «Les structures sépulcrales de la nécropole de Vignely (Seine-et-Marne)", in Chambon P., LeClerC J. (DIR.), Les Pratiques funéraires néolithiques avant 3500 av. J.-C. en France et dans les régions limitrophes, Actes de la table ronde de la SPF, Saint-Germain-en-Laye, juin 2001, Paris, SPF (coll. Mémoires de la SPF, 33), p. $159-173$.

Chambon P., Mordant D., Pariat J.-G. 2009: «Sépultures du Néolithique moyen en Bassin parisien: le cas des architectures sépulcrales ", in LE BRUN-RICALENS F., VAlotteau F., HaUzeUr A. (DIR.), Relations interrégionales au Néolithique entre Bassin parisien et Bassin rhénan, Actes du $26^{e}$ colloque interrégional sur le Néolithique, Luxembourg, 8-9 nov. 2003, Luxembourg, Musée national d'histoire et d'art (coll. Archaeologia Mosellana, 7, 2007), p. 445467.

Chambon P., Pétillon J.-M.

2009: «Des chasseurs Cerny?», BSPF, 106, 4, p. $761-783$.

\section{Clemente I.}

1998: El "lustre de suelo" y la conservación de los micropulidos de uso: resultados experimentales para una aplicación arqueológica, La Rogère 1, Locus IV, Mémoire de stage postdoctoral, Generalitat de Catalunya, $45 \mathrm{p}$.

Cleuvenot E., Houët F.

1993: «Proposition de nouvelles équations d'estimation de stature applicables pour un sexe indéterminé et basées sur les échantillons de Trotter et Gleser", $B M S A P, 5$, p. 245-255.

Crubézy e., Sellier P.

1990: «Caractères discrets et organisation des ensembles sépulcraux", BMSAP, 2 (3-4), p. 171-178.
Crubézy E., Telmon N., Sevin A., PICARD J., ROUGÉ D., LARROUY G., Braga J., Ludes B., MURail P.

1999: «Microévolution d'une population historique: étude des caractères discrets de la population de Missiminia (Soudan, III ${ }^{\mathrm{e}}-\mathrm{VI} \mathrm{I}^{\mathrm{e}}$ siècle) ", BMSAP, 11 (1-2), p. 1-213.

\section{DELOR J.-P.}

1983: Compte rendu de fouille de sondage effectuée sur la commune de Chichery (89): "Sur les Pâtureaux», Rapport d'opération de fouille, Dijon, SRA de Bourgogne, $40 \mathrm{p}$.

1984: Compte rendu de fouille de sondage effectuée sur la commune de Chichery (89): «Sur les Pâtureaux», Rapport d'opération de fouille, Dijon, SRA de Bourgogne, 111 p. et 2 doc. h. $\mathrm{t}$.

1991: "L'habitat néolithique de Gurgy, "les Plantes du Mont", 1989-1990 (note préliminaire) », $B S P F, 88,1$, p. 18-20.

1995: Archéologie et carrières de granulats de l'Yonne: bilan 1994, Dijon, SRA de Bourgogne, $89 \mathrm{p}$.

\section{DUDAY H.}

1995: «Anthropologie de "terrain", archéologie de la mort", in JOUSSAUME R. (DIR.), La Mort: passé, présent, conditionnel, Actes du colloque de La Roche-sur-Yon, juin 1994, La Roche-sur-Yon, Groupe vendéen d'études préhistoriques, p. 33-58.

Duday H., Lambach F., Plouin S.

1990: "Contribution de l'anthropologie de terrain à l'interprétation architecturale d'un ensemble funéraire: la tombe $12 \mathrm{du}$ tumulus 2A à Nordhouse (Bas-Rhin)», Nouvelles de l'archéologie, 40, p. 15-18.

\section{DUHAMEL $P$.}

2004: «La nécropole monumentale d'Escolives-Sainte-Camille "la Pièce de l'Étang" (Yonne): premiers résultats (fouilles 1997-1999) », in Internéo 5, Actes de la journée d'information du 20 nov. 2004, Paris, SPF, p. 63-72.

2006: «Vases "intrus" ? Vases "bâtards" ? de quelles influences témoignent les poteries retrouvées en milieu funéraire dans le bassin d'Yonne au début du Néolithique moyen ?» in BARAY L. (DIR.), Artisanats, sociétés et civilisations, Hommage à Jean-Paul Thévenot, Dijon, Société archéologique de l'Est (coll. Suppl. à la $R A E, 24)$, p. 115-131.

DUHAMEL P. avec la collab. de Fonton M., CARRE H.

1997: «La nécropole monumentale Cerny de Passy (Yonne): description d'ensemble et problèmes d'interprétation", in Constantin C., Mordant D., SIMONIN D. (DIR.), La Culture de Cerny: nouvelle économie, nouvelle société au Néolithique, Actes du colloque international de Nemours, 1994, Nemours, APRAIF (coll. MMPIF, 6), p. 397-448.

\section{FINNEGAN M.}

1978: «Non-Metric Variation of the Infracranial Skeleton ", Journal of Anatomy, 125, 1, p. 23-37.

\section{FORRER R.}

1911: «Das neolitische Gräberfeld bei Lingolsheim und die verwandten Gräber von Erstein, Wolfisheim, etc. verglichen mit unseren bandkeramischen Gräbern ", Anzeiger für Elsässische Altertumskunde, I, p. $149-171$ et p. $189-204$.

1938: «Le cimetière néolithique de Lingolsheim à poteries poinçonnées, au crâne trépané et aux tombes de la zone rubanée", Cahiers d'archéologie et d'histoire d'Alsace, VII, p. 191-206.

\section{GeMmerich PFister I. G. L.}

1999: Création d'une collection anthropologique de référence et application des caractères discrets dans le cas de généalogies connues, Thèse de Doctorat, Université de Genève, $266 \mathrm{p}$.

\section{Gibaja BAO J. F}

2003: Comunidades Neolíticas del Noreste de la Peninsula Ibérica: una aproximación socioeconómica a partir del estudio de la función de los útiles líticos, Oxford, Archaeopress (coll. British Archaeological Reports, International Series, S1140), $318 \mathrm{p}$.

HaUser G., De STEPHANo G. F.

1989: Epigenetic Variants of the Human Skull, Stuttgart, Schweizerbart, $301 \mathrm{p}$.

\section{JEUNESSE C.}

1997: Pratiques funéraires au Néolithique ancien: sépultures et nécropoles danubiennes, 55004900 av. J.-C., Paris, éd. Errance (coll. Hespérides), 168 p.

KAMINA P. avec la collab. de ZWOBADAKAMINA A.

1983: Dictionnaire atlas d'anatomie, 3 vol., Paris, Maloine, $1841 \mathrm{p}$.

\section{KASA M.}

2002: Paläopathologische Untersuchungen an dem Zahnmaterial des mittelneolithischen Gräberfeldes von Jechtingen am Kaiserstuhl, Dissertation pour l'obtention du grade de docteur en médecine dentaire, AlbertLudwigs-Universität Freiburg, 241 p. 


\section{LEDERMANN S.}

1969: Nouvelles tables-types de mortalité, Paris, Ined (coll. Les Cahiers de l'Ined, 53), $260 \mathrm{p}$.

\section{LEVI-SALA I.}

1993: «Use-Wear Traces: Processes of Development and Post-Depositional Alterations ", in ANDERSON P.C., Beyries S., OTTE M., Plisson H. (DIR.), Traces et fonction: les gestes retrouvés, Actes du colloque international de Liège, 8-10 déc. 1990, Université de Liège (coll. Études et recherches archéologiques de l'université de Liège, 50), p. 401-416.

\section{LOUbOUTIN C., SIMONIN D.}

1997: «Le Cerny-Videlles: un faciès ancien de la culture de Cerny", in CONSTANTIN C., MORDANT D., SIMONIN D. (DIR.), La Culture de Cerny: nouvelle économie, nouvelle société au Néolithique, Actes du colloque international de Nemours, 1994, Nemours, APRAIF (coll. MMPIF, 6), p. 135-137.

\section{MASSET C.}

1987: «Le "recrutement" d'un ensemble funéraire", in DUDAY H., MASSET C. (DIR.), Anthropologie physique et archéologie: méthodes d'étude des sépultures, Actes du colloque de Toulouse, 4-6 nov. 1982, Paris, CNRS Éditions, p. 111-134.

Moorrees C. F. A., FANNing E. A.,

HUNT E. E. JR

1963a: «Age Variation of Formation Stages for Ten Permanent Teeth", Journal of Dental Research, 42, p. 1490-1502.

1963b: «Formation and Resorption of Three Deciduous Teeth in Children", AJPA, 21(2), p. 205-213

\section{MORDANT D.}

1987: «Des inhumations en "pleine terre" ?: l'exemple de la Petite-Seine", in DUdAY H., MASSET C. (DIR.), Anthropologie physique et archéologie: méthodes d'étude des sépultures, Actes du colloque de Toulouse, 4-6 nov. 1982, Paris, CNRS Éditions, p. 155-165.

1997: «Le complexe des Réaudins à Balloy: enceinte et nécropole monumentale", in Constantin C., MORdant D. SIMONIN D. (DIR.), La Culture de Cerny: nouvelle économie, nouvelle société au Néolithique, Actes du colloque international de Nemours, 1994, Nemours, APRAIF (coll. MMPIF, 6), p. 449-479.

\section{MORDANT C., MORDANT D.}

1978: «Les sépultures néolithiques de Noyensur-Seine (Seine-et-Marne)», BSPF, 75 (coll. Études et travaux, 11-12), p. 559578 .

MORDANT C., MORDANT D. avec la collab. de Poulain-Josien T.

1970: «Le site néolithique des Gours-auxLions à Marolles-sur-Seine (Seineet-Marne)», BSPF, 67, (coll. Études et travaux, 1), p. 345-372.

Müller F., Duhamel P., Augereau A., DEPIERRE G. avec la collab. de JACQUEMIN M., POYETON A., SIDÉRA I., Poulain T., CHARmot A.

1997: «Une nouvelle nécropole monumentale Cerny à Gron, "les Sablons" (Yonne) ", in JEUNESSE C. (DIR.), Le Néolithique danubien et ses marges entre Rhin et Seine, Actes du $22^{e}$ colloque interrégional sur le Néolithique, Strasbourg, 27-29 oct. 1995, Zimmersheim, APRAA (coll. Suppl. aux Cahiers de l'APRAA), p. 103-133.

\section{MURAIL P., BRUZEK J., BRAGA J.}

1999: «A New Approach to Sexual Diagnosis in PastPopulations: Pratical Adjustements from Van Vark's Procedure ", International Journal of Osteoarchaeology, 9, 1, p. 39-53.

MURail P., BRUZek J., HouËt F., CUnha E. 2005: «DSP: a Tool for Probabilistic Sex Diagnosis Using Worldwide Variability in Hip-Bone Measurements", BMSAP, 17, 3-4, p. 167-176.

\section{OLIVIER G.}

1960: Pratique anthropologique, Paris, Vigot, $299 \mathrm{p}$.

Olivier G., Pineau H.

1958: "Détermination de l'âge du fotus et de l'embryon", Archives d'anatomie (La Semaine des hôpitaux), 6, p. 21-28.

OWINGS WebB P. A., SUCHeY MYeRS J.

1985: «Epiphyseal Union of the Anterior Iliac Crest and Medial Clavicle in a Modern Multiracial Sample of American Males and Females », AJPA, 68, 4, p. 457-466.

\section{Pellet P.}

1978: «La nécropole rubanée de "l’Étang David" à Chichery (Yonne) ", RAE, 29, $1-2$, p. $65-84$

\section{Pellet C., Delor J.-P.}

1981a: «Sur une structure circulaire découverte au "Crot au Moine" commune de Beaumont, Yonne: note préliminaire», in Le Néolithique de l'est de la France, Actes du colloque interrégional sur le Néolithique, Sens,
27-28 sept. 1980, Sens, Société archéologique de Sens, p. 155-161

1981b: "L'enceinte à fossé interrompu du "Crot au Moine", commune de Beaumont, Yonne: note préliminaire», in Le Néolithique de l'est de la France, Actes du colloque interrégional sur le Néolithique, Sens, 27-28 sept. 1980, Sens, Société archéologique de Sens, p. 207-209.

POPLIN F.

1975: «Problèmes d'ostéologie», in LEROIGOURHAN A. (DIR.), Séminaire sur les structures d'habitat: sépultures, Paris, Collège de France, p. 27-34.

\section{Prestreau M.}

2006: «Le complexe chasséen dans l'Yonne», in BARAY L. (DIR.), Artisanats, sociétés et civilisations, Hommage à Jean-Paul Thévenot, Dijon, Société archéologique de l'Est (coll. Suppl. à la $R A E, 24)$, p. 87-114.

\section{PRESTREAU M., ThÉVENOT J.-P.}

1996: «L'axe Yonne-Armançon-Saône au Néolithique moyen: cadre chronologique et interprétations culturelles", in DUhAMEL P. (DIR.), La Bourgogne entre les bassins rhénan, rhodanien et parisien: carrefour ou frontière? Actes du XVIII ${ }^{e} \mathrm{col}$ loque interrégional sur le Néolithique, Dijon, 25-27 oct. 1991, Dijon, Société archéologique de l'Est (coll. Suppl. à la RAE, 14), p. $383-396$

REIMER P. J. ET AL.

2004: «IntCal04 Terrestrial Radiocarbon Age Calibration, 0-26 cal kyr BP», Radiocarbon, 46, 3, p. 1029-1058.

\section{ROTTIER S.}

2007: «L'architecture funéraire des sépultures du Néolithique moyen des Noisats à Gurgy (Yonne, France) ", in MOINAT P., Chambon P. (DIR.), Les Cistes de Chamblandes et la place des coffres dans les pratiques funéraires du Néolithique moyen occidental, Actes du colloque de Lausanne, 12-13 mai 2006, Paris, SPF (coll. Mémoires de la SPF, 43) et Lausanne, Musée d'archéologie et d'histoire du canton de Vaud (coll. Cahiers d'archéologie romande, 110), p. 99-107.

\section{RotTier S., MORdant C., CHAMbon P.,} THEVENET C.

2005: «Découverte de plus d'une centaine de sépultures du Néolithique moyen à Gurgy, "les Noisats" (Yonne) », BSPF, 102 , 3, p. 641-645. 


\section{SAUNDERS S. R.}

1978: The Development and Distribution of Discontinuous Morphological Variation of the Human Infracranial Skeleton, Ottawa, National Museum of Man (coll. Archeaological Survey of Canada, 81).

\section{SCHEUER L., BLACK S.}

2000: Developmental Juvenile Osteology, Londres, Academic Press.

\section{SCHMitT A.}

2005: «Une nouvelle méthode pour estimer

l'âge au décès des adultes à partir de la surface sacro-pelvienne iliaque », BMSAP, 17, 1-2, p. 279-302.

SCOTT G. R., TURner II C. G.

1997: The Anthropology of Modern Human Teeth: Dental Morphology and its Variation in Recent Human Populations, Cambridge, Cambridge University Press, 406 p.

\section{SELLIER P.}

1996: «La mise en évidence d'anomalies démographiques et leur interprétation: population, recrutement et pratiques funéraires du tumulus de Courtesoult», in PININGRE J.-F. (DIR.), Nécropoles et société au premier âge du Fer: le tumulus de Courtesoult (Haute-Saône), Paris, MSH (coll. DAF, 54), p. 188-202.
SEMPÉ M., PEDRON G.

1979: Auxologie, méthode et séquences, Paris, Laboratoire Théraplix, 205 p.

\section{SIMONIN D., BACH S., RICHARD G.,}

\section{VINTROU J.}

1997: «Les sépultures sous dalle de type Malesherbes et la nécropole d'Orville", in Constantin C., Mordant D., SIMONIN D. (DIR.), La Culture de Cerny: nouvelle économie, nouvelle société au Néolithique, Actes du colloque international de Nemours, 1994, Nemours, APRAIF (coll. MMPIF, 6), p. 341-379.

\section{SMITH B. H.}

1984: «Patterns of Molar Wear in HunterGatherers and Agriculturalists ", AJPA, 63,1 , p. $39-56$.

\section{SPATZ H.}

1999: Das mittelneolithische Gräberfeld von Trebur, Kreis Gross-Gerau, Wiesbaden, Landesamt für Denkmalpflege Hessen (coll. Materialien zur Vor- und Frühgeschichte von Hessen, 19), vol. 1: texte et vol. 2: catalogue, biblio. et pl., 692 p., 298 fig., 148 tabl., 188 pl., 1 pl. h. t.

\section{TAINTURIER J.-L.}

1981: «Fouilles archéologiques à Chichery (Yonne)", Rapport d'opération de fouille, SRA de Bourgogne, 6 p., 4 fig., 14 ph. h. t.

\section{THÉVENOT J.-P.}

1982: «Informations archéologiques, Circonscription de Bourgogne", Gallia Préhistoire, 25, 2, p. 311-356.

1985: «Informations archéologiques, Circonscription de Bourgogne", Gallia Préhistoire, 28, 2, p. 171-210.

1991 : «Remarques préliminaires sur l'industrie lithique des niveaux chasséens de Chassey (la Redoute) », in BEECHING A., BINDER D., BLANCHET J.-C., Constantin C., Dubouloz J., MARTINEZ R., MORDANT D., THÉVENOT J.-P., VAQUER J. (DIR.), Identité du Chasséen, Actes du colloque international de Nemours, 1989, Nemours, APRAIF (coll. MMPIF, 4), p. 251-259.

THÉVENOT J.-P. (DIR.)

2005: Le Camp de Chassey: Chassey-le-Camp, Saône-et-Loire, les niveaux néolithiques du rempart de «la Redoute», Dijon, Société archéologique de l'Est (coll. Supl. à la $R A E, 22), 464$ p.

Trotter M., Gleser G. C.

1958: "A Re-Evaluation of Estimation of Stature Based on Measurments of Stature Taken during Life and Long Bones after Death », AJPA, 16, p. 79-124

\section{VILLOTTE S.}

2006: «Connaissances médicales actuelles, cotation des enthésopathies: nouvelle méthode », BMSAP, 18, 1-2, p. 65-85. 